\title{
Identification and Analysis of Gene Product Modifiers of $\alpha$-Synuclein Toxicity in the Fruit Fly (D. melanogaster)
}

\author{
Dissertation \\ for the award of the degree \\ "Doctor of Philosophy" (Ph.D.) \\ Division of Mathematics and Natural Sciences \\ of the Georg-August-Universität Göttingen
}

\author{
submitted by \\ Erin Butler \\ from Cornwall, Canada \\ Göttingen 2010
}


Prof. J.B. Schulz (1. Reviewer)

Neurodegeneration and Restorative Research, University of Göttingen/ Neurology Clinic, University Clinic Aachen

Prof. M. Simons (2. Reviewer)

Cellular Neuroscience, Max Planck Institute for Experimental Medicine/ Dept. of Neurology, University of Göttingen

Prof. R. Heinrich (3. Reviewer)

Neurobiology, Johann-Friedrich-Blumenbach Institute for Zoology and

Anthropology, University of Göttingen

Prof. H. Jäckle (4. Reviewer)

Molecular Developmental Neurobiology, Max Planck Institute for Biophysical Chemistry

Oral examination: September 8, 2010 
I hereby declare that the thesis 'Identification and Analysis of Gene Product Modifiers of $\alpha$ Synuclein Toxicity in the Fruit Fly (D. melanogaster)' has been written independently and using no other sources other than those quoted.

Erin Butler

Gottingen, July 29, 2010 


\section{Acknowledgements}

No project is completed without the assistance of others. I would like to take this opportunity to thank some of the many people who provided guidance, advice and technical support over the course of my doctoral work.

In particular, I would like to thank:

Prof. Jörg Schulz for supervision and guidance of my project.

Dr. Aaron Voigt, for supervision and fly expertise.

Dr. Ellen Gerhardt, for viral vector production and having the patience to answer many questions.

Christiane Fahlbusch, for excellent technical support.

Dr. Peter Karsten, for assistance with plasmids, IPs and co.

Dr. Anja Drinkut, for moral support, optimism and a super trip to Korea.

The entire Department of Neurodegeneration, for providing a supportive work environment. 


\section{$\underline{\text { Table of Contents }}$}

i. Abbreviations

I. Abstract

II. Introduction 2

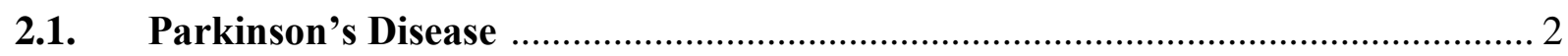

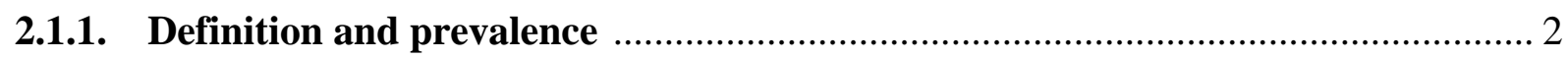

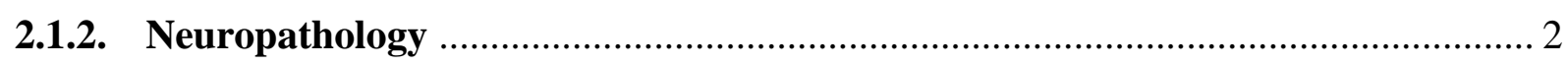

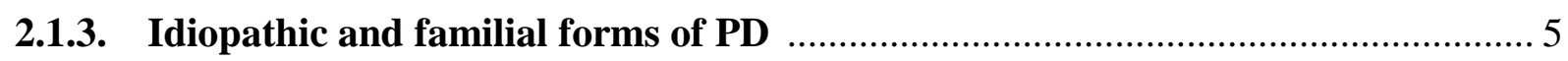

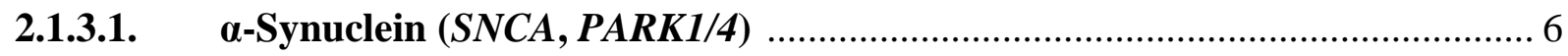

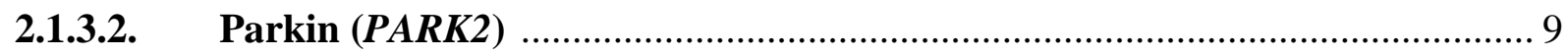

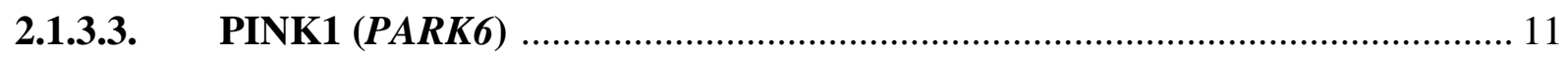

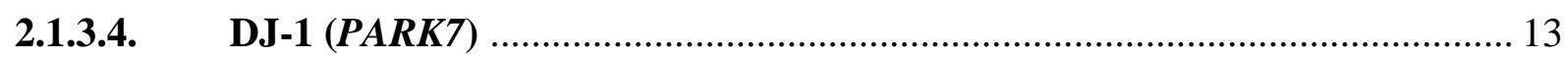

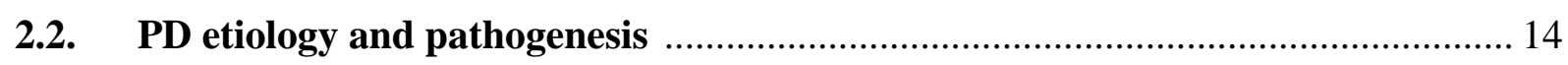

2.2.1 UPS dysfunction and protein aggregation ...................................................... 14

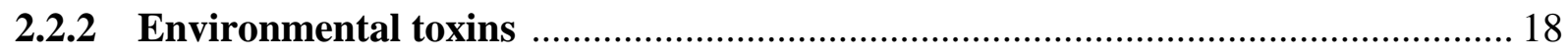

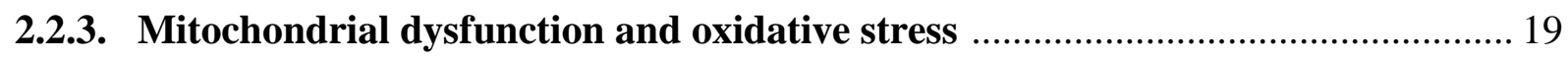

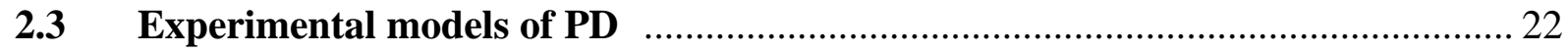

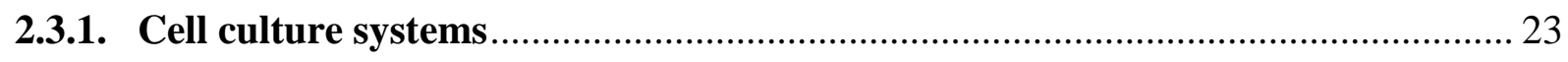

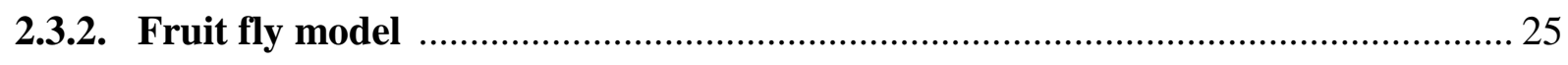

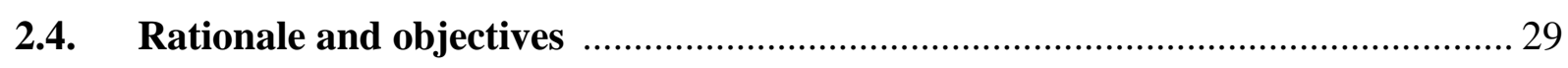

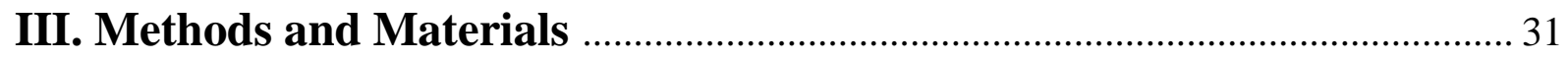

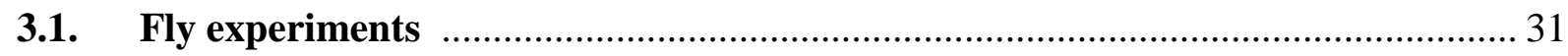

3.1.1. Fly stocks and transgenic fly expressing human TRAP1 creation ..................... 31

3.1.2. Single fly polymerase chain reaction (PCR) for genotyping ............................... 33

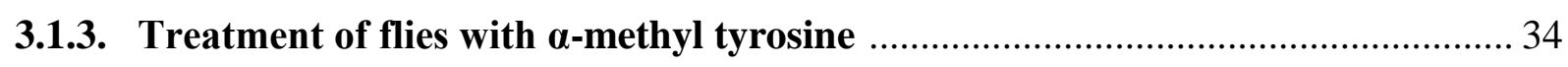

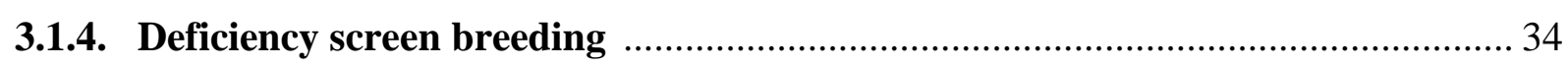

3.1.5. Measurement of fly brain DA using HPLC assays ......................................... 36

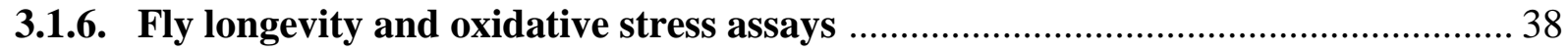

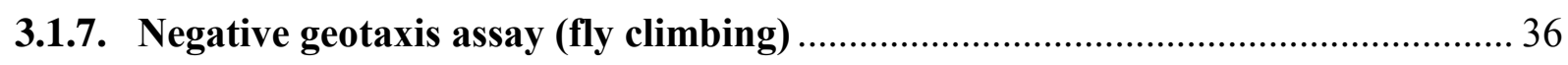

3.1.8. Whole fly brain stain for tyrosine hydroxylase and neuron counting ….............. 37 


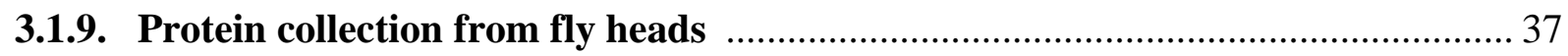

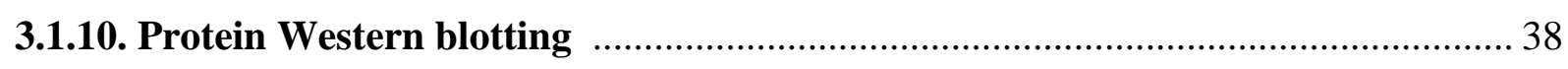

3.1.11. Total RNA isolation and cDNA preparation from fly heads .............................. 39

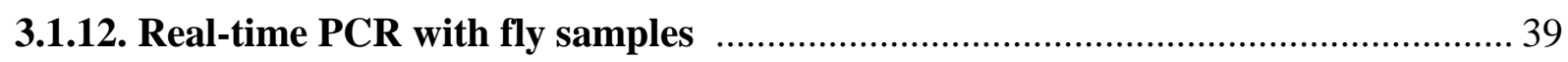

3.1.13. Measurement of citrate synthase (CS) enzymatic

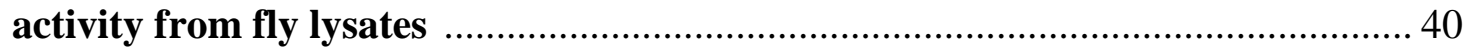

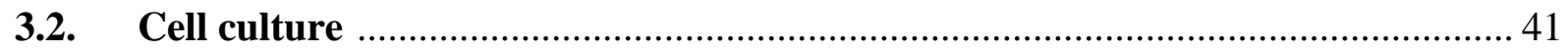

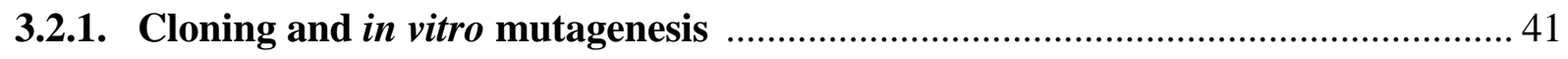

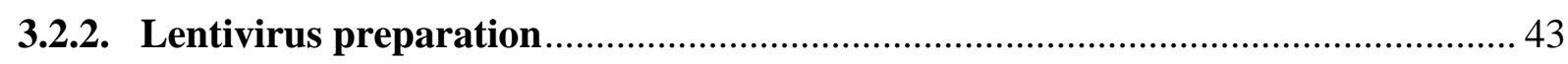

3.2.3. Cells, plasmid transfection and viral infection ................................................ 43

3.2.4. Total RNA from cell culture and real time PCR ............................................. 44

3.2.5. Protein collection from cell culture and Western blotting ................................... 45

3.2.6. Oxidative stress testing and measurement of cell viability ATP synthesis

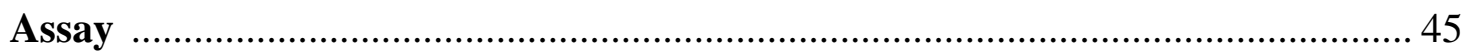

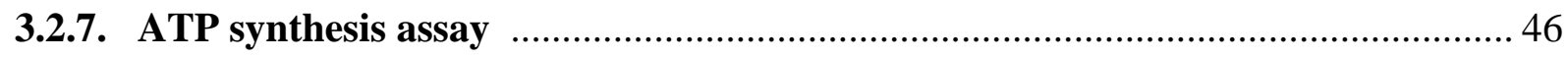

3.2.8. Measurement of steady state cellular ATP ..................................................... 46

3.2.9. Assessment of mitochondrial membrane potential ........................................ 47

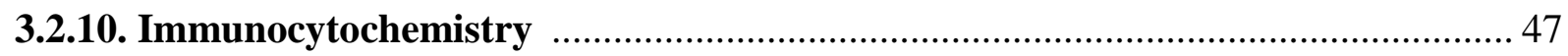

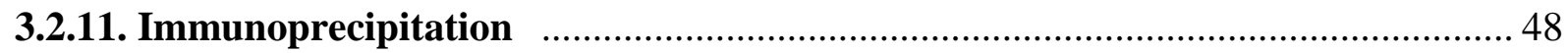

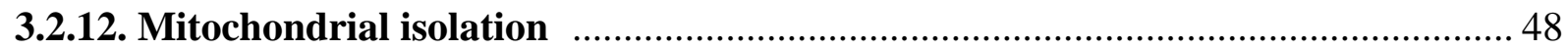

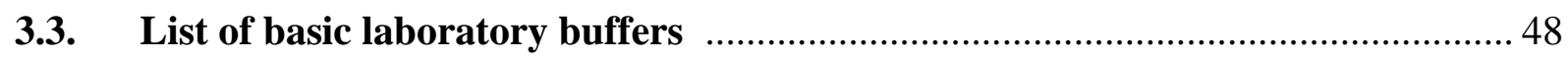

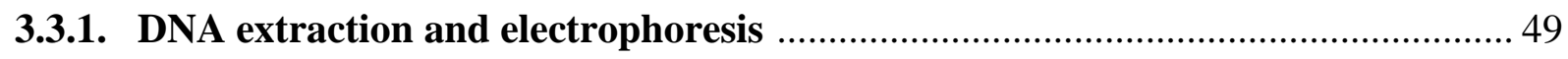

3.3.1. Protein extraction and Western blotting ......................................................... 49

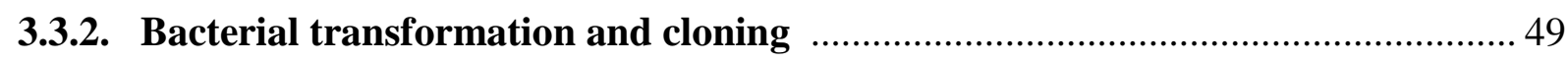

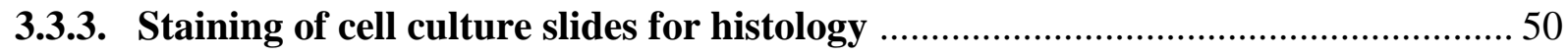

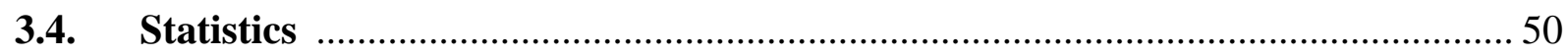

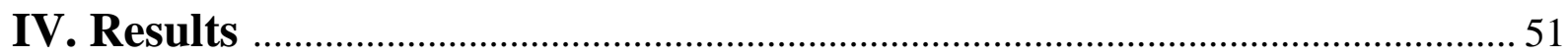

4.1. Initial Experiments: Fly Model Establishment and Genetic Screening

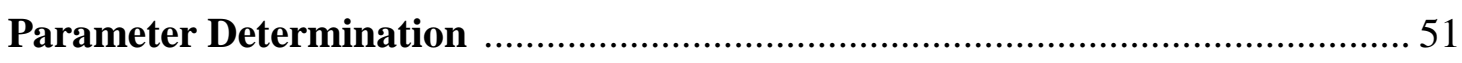

4.1.1. Generation of recombined ddc $>$ and elav $>\alpha$-Synuclein[A53T]-expressing flies 51

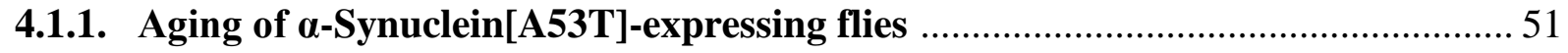

4.1.2. Determination of appropriate sample size number for HPLC fly brain DA measurements 
4.1.3. Influence of time of day on DA content in fly heads 53

4.1.4. Variation in DA content between fly lines

4.1.5. Measurement of fly head DA over time following tyrosine hydroxylase (TH) inhibition ( $\alpha$-methyl tyrosine (MT) treatment)

4.1.6. Measurement of DA with age in control and $\alpha$-Synuclein[A53T]expressing flies 53

4.1.7. Loss of dopaminergic neurons in flies expressing $\alpha$-Synuclein[A53T] 56

4.1.8. Data Summary from Initial Experiments to Establish the Model 59

4.2. Primary Deficiency Screen Data (HPLC measurement of fly head DA) 59

4.2.1. Data Summary from Primary Deficiency Screen 64

4.3. Modification of $\alpha$-Synuclein[A53T] Toxicity by Mitochondrial Chaperone Protein, TRAP1

4.3.1. Modification of $\alpha$-Synuclein[A53T] Toxicity by TRAP1 in D. melanogaster 64

4.3.1.1. Effect of human TRAP1 overexpression on loss of brain DA in the fly 64

4.3.1.2. Sensitivity to oxidative stress in flies expressing $\alpha$-Synuclein[A53T] and alteration to TRAP1 expression

4.3.1.3. Negative geotaxis assay of flies expressing $\alpha$-Synuclein[A53T] with alteration to

TRAP1 expression

4.3.1.4. Loss of DA neurons in flies expressing $\alpha$-Synuclein[A53T] and alteration to TRAP1 expression

4.3.1.5. Longevity of flies expressing $\alpha$-Synuclein[A53T] with alteration to TRAP1 expression

4.3.2. Modification of $\alpha$-Synuclein[A53T] Toxicity by TRAP1 in Human HEK293 Cell

Culture 71

4.3.2.1. Knockdown and overexpression of TRAP1 in $\alpha$-Synuclein[A53T] expressing cells

4.3.2.2. $\alpha$-Synuclein[A53T] mitochondrial localization using immunofluorescence and confocal microscopy

4.3.2.3. $\alpha$-Synuclein[A53T] mitochondrial localization using cell fractionation 76

4.3.2.4. Oxidative stress sensitivity of HEK293 cells expressing $\alpha$-Synuclein[A53T],

TRAP1 and/or siTRAP1

4.3.2.5. $\alpha$-Synuclein[A53T] mitochondrial toxicity: measurement of ETC complex ATP production 79 
4.3.2.6. $\alpha$-Synuclein[A53T] mitochondrial toxicity: measurement of total ATP content 81

4.3.2.7 $\alpha$-Synuclein[A53T] mitochondrial toxicity: loss of mitochondrial membrane

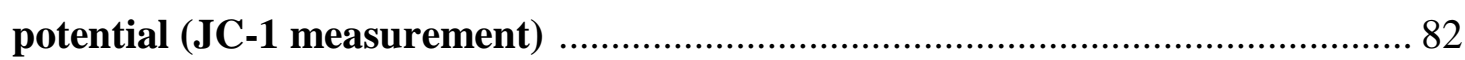

4.3.2.8. Measurement of total mitochondrial protein in HEK293 cells expressing $\alpha$ -

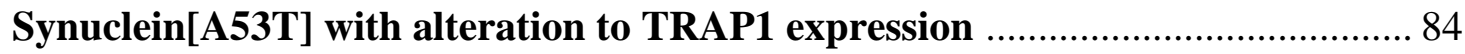

4.3.2.9. Protein-protein interaction of $\alpha$-Synuclein[A53T] and TRAP1 ......................... 84

4.3.2.10. Creation of TRAP1 mutants and expression in HEK293 cells ................. 85

4.3.2.11. Phosphorylation state of TRAP1 and TRAP1 mutants ............................ 87

4.3.2.12. Effect of TRAP1 mutation on protein-protein interaction with $\alpha$ -

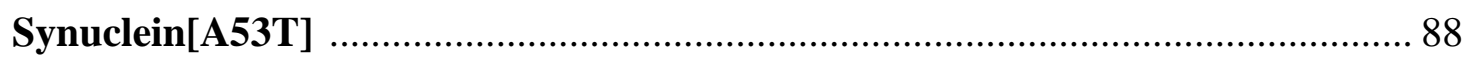

4.3.2.13. Effect of TRAP1 mutation on TRAP1 oxidative stress rescue effect ........ 89

4.3.2.14. Effect of TRAP1 mutations on ATP production of ETC complexes ......... 90

4.3.2.15. Effect of TRAP1 mutation on mitochondrial membrane potential .......... 92

4.3.2.16. Effect of TRAP1 mutation on total cellular ATP ................................... 93

4.3.2.17. Effect of TRAP1 mutation on total mitochondrial protein ...................... 94

4.3.3. Modification of $\alpha$-Synuclein[A53T] Toxicity by TRAP1 in Rat Primary Cortical

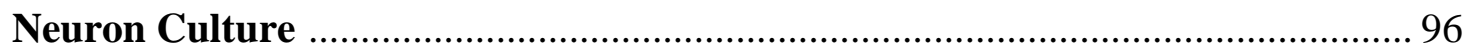

4.3.3.1.Overexpression of TRAP1 and $\alpha$-Synuclein[A53T] in primary cortical neurons

4.3.3.2. Confirmation of neuronal phenotype and viral infection in rat primary cortical culture

4.3.3.3. Rotenone treatment of primary cortical neurons

4.3.4. Data Summary from Investigation into TRAP1 modification of $\alpha$ Synuclein[A53T] Toxicity

4.4. Secondary Screen for Mitochondrial Related Genes Modifying $\alpha$-Synuclein[A53T] Toxicity

V. Discussion 102

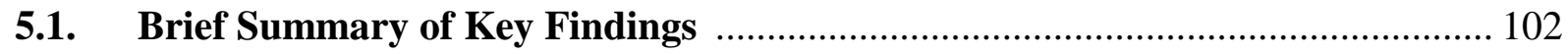

5.2. Genetic Screen Results 103

5.2.1. Limitations of the primary and secondary genetic screens for modifiers of $\alpha$ Synuclein[A53T] neurotoxicity

5.2.2. Comparison of candidates to previously identified $\alpha$-Synuclein modifiers and genetic screens 
5.2.3. Secondary mitochondrial-related screen: discussion of targets generated 108

5.3. TRAP1 modification of $\alpha$-Synuclein[A53T] toxicity in Drosophila, HEK293 cells and primary neurons: conclusions, limitations and unanswered questions 109

5.4. Significance of TRAP1- $\alpha$-Synuclein[A53T] interaction in the context of PD Pathogenesis 120

A1. Appendix Methods 123

A2. Appendix Results 126

VI. References 144

Curriculum Vitae 163 


\section{i. Abbreviations}
AD- Alzheimer's disease
ADP- adenosine diphosphate
ATP- adenosine triphosphate
BSA- bovine serum albumin
CCCP- carbonyl cyanide $m$-chlorophenylhydrazone
CMA- chaperone-mediated autophagy
CS- citrate synthase
CSP- $\alpha$ - cysteine string protein- $\alpha$
DA- dopamine
DAT- DA transporter

Ddc- dopa decarboxylase

DMSO- dimethylsulfoxide

DNA- deoxyribonucleic acid

DTNB- 5, 5'-Dithio-Bis -2-Nitrobenzoic Acid

EDTA- Ethylendiamine-tetraacetate

ER- endoplasmic reticulum

ETC- electron transport chain

GFP-green fluorescent protein

GTP- guanosine triphosphate

HEK293- human embryonic kidney-293 cells

HEPES- 4-(2-hydroxyethyl)-1-piperazineethanesulfonic acid

HPLC- high performance liquid chromatography

IP- immunoprecipitation

KO- knock out

LB- Lewy Body 
L-DOPA- levodopa (L-3,4-Dihydroxyphenylalanine)

mTOR- multiple Rheb-target-of rapamycin

MAPKKK- mitogen- activated protein kinase kinase kinase

MEF2D- myocyte enhancer factor-2D

MPTP- 1-methyl-1,4-phenyl-1,2,3,6-tetrahydropyridine

NAC- non-amyloid component

OAA- oxaloacetic acid

PBS- phosphate buffered saline

PBT- PBS/ $0.1 \%$ Triton $\mathrm{X}$

PCR- polymerase chain reaction

PD- Parkinson's disease

PFA- paraformaldehyde

PINK1- PTEN-induced kinase- 1

PTP- permeability transition pore

RNA- ribonucleic acid

RNAi- RNA interference

ROS- reactive oxygen species

rpm- revolutions per minute

RT- room temperature

s- seconds

siRNA- small interfering RNA

SNARE- SNAP (Soluble NSF Attachment Protein) Receptors

SNc- substantia nigra pars compacta

SOD- superoxide dismutase

SUMO- small ubiquitin-related modifier

TAE- Tris, acetic acid, EDTA buffer 
TH- tyrosine hydroxylase

TRAP1- tumor necrosis factor receptor-associated protein 1

UAS- upstream activating sequence

UBCH7- ubiquitin-conjugating enzyme

UPS- ubiquitin-proteasome system

vs.- versus

wt- wild type 


\section{Abstract}

Parkinson's disease (PD) is a progressive neurodegenerative disorder, pathologically characterized by loss of dopaminergic neurons in the substantia nigra pars compacta. Various mutations in $\alpha$-Synuclein $(S N C A, P A R K 1 / 4)$ result in autosomal dominant, inherited PD. Overexpression or mutation of $\alpha$-Synuclein is associated with protein aggregation and results in disturbance to a number of cell systems, including the ubiquitin-proteasome system and mitochondria. To identify new genetic modifiers of $\alpha$-Synuclein neurotoxicity, fruit flies (Drosophila melanogaster) expressing human $\alpha$-Synuclein with the familial PD mutation [A53T] in dopaminergic neurons, in combination with a specific chromosomal deletion, were screened for aging-dependent loss of brain dopamine (DA) using high performance liquid chromatography. Sub-screening within deficiencies for individual genes using P-element gene disruption and RNA interference fly lines revealed that decreased expression of the mitochondrial chaperone protein, tumor necrosis factor receptor associated protein-1 (TRAP1), enhanced $\alpha$-Synuclein[A53T] neurotoxicity. Flies showed an enhanced loss of brain DA, dopaminergic neurons and climbing ability with time. In addition, sensitivity to oxidative stress treatment (paraquat or hydrogen peroxide) was enhanced. Overexpression of human TRAP1 rescued these phenotypes. Similarly, coincident overexpression of both human TRAP1 and $\alpha$-Synuclein[A53T] in rat primary cortical neurons rescued $\alpha$-Synuclein-induced sensitivity to rotenone treatment. In human embryonic kidney- 293 (HEK293) cells, small interfering RNA directed against TRAP1 enhanced $\alpha$ Synuclein[A53T] -induced sensitivity to hydrogen peroxide or rotenone. TRAP1 overexpression provided rescue. $\alpha$-Synuclein[A53T] was localized to the mitochondria using cell fractionation and co-localization of fluorescent signals. $\alpha$-Synuclein[A53T] can directly interfere with mitochondrial function, as it was found to inhibit Complex I activity and when in combination with siTRAP1, decreased mitochondrial membrane potential. These effects could be blocked by TRAP1 overexpression. A direct protein-protein interaction between TRAP1 and $\alpha$ Synuclein[A53T] was found using immunoprecipitation. In addition, data from the primary deficiency screen was cross referenced with candidate gene data from a previously published Drosophila mitochondrial modifier screen. Seventeen additional candidate genes modifying $\alpha-$ Synuclein[A53T] toxicity in the fly, including Tiny Tim 50, bellweather and p70 S6k, were identified. These results show that $\alpha$-Synuclein[A53T] toxicity is intimately connected to that of mitochondrial function and that rescue from toxicity in fly, rat primary neurons and human cell line can be achieved using overexpression of the mitochondrial chaperone TRAP1. 


\section{Introduction}

\subsection{Parkinson's Disease}

\subsubsection{Definition and prevalence}

First formally described by Dr. James Parkinson in 1817, Parkinson's disease (PD) is the second most prevalent neurodegenerative disease behind Alzheimer's (AD). The disease is clinically characterized by a constellation of movement-related symptoms including unilateral tremor at rest, bradykinesia (slowness of movement), akinesia (inability to move) and postural instability. Though primarily defined as a movement disorder, late features of the disease can include a wide range of non-motor symptoms including cognitive impairment, hallucinations, sleep disturbances and autonomic dysfunction including constipation, hypotension and sexual dysfunction. ${ }^{1}$

Age of PD onset is typically 60 years and age is the primary risk factor. For people above the age of 50, the incidence of PD is approximately $110-300$ per 100,000 . However above age 80 , the incidence rate climbs to $400-500$ per $100,000 .^{2}$ Men are twice as likely to develop the disease than women: total lifetime probability for developing the disease is $2.0 \%$ for men and $1.3 \%$ for women. ${ }^{3}$

\subsubsection{Neuropathology}

$\mathrm{PD}$ is characterized by degeneration of selective brain regions. In particular, substantial cell death is found within the substantia nigra pars compacta ( $\mathrm{SNc}$ ) located in the midbrain basal ganglia. SNc depigmentation occurs due to the death of dopaminergic, neuromelanin- containing neurons. ${ }^{4}$ The major efferent projection of the $\mathrm{SNc}$ is the nigrostriatal projection, which supplies the striatum with DA input. Thus, SNc cell loss results a reduction of striatum DA content. Key functions of the $\mathrm{SNc}$ and striatum include motor planning and control, and thus dysfunction of these brain regions results in the akinesia and rigidity of PD. Onset of motor symptoms occurs with approximately $80 \%$ reduction of the 


\section{INTRODUCTION}

striatum DA content, corresponding to $60 \%$ cell death of the SNc DA neurons. ${ }^{1}$ Importantly, a withdrawal of SNc projections to the striatum is observed in advance of cell body death in the SN. ${ }^{5}$ Thus, the first subtle motor deficits of PD most likely result from this loss in DA content, in advance of large scale SNc cell death. Neuronal loss is associated with aging and has been observed to occur in dorsomedial SN. However, PD cell death occurs within the ventrolateral and caudal SN. Thus, the pattern of cell death in the SNc is specific to PD pathology and not typical of aging. ${ }^{6,7}$ Curiously, the DA neurons of the SNc seem particularly vulnerable to degeneration. That is, not all brain DA neurons show such marked degeneration as those found within the SNc. For example, DA cells located in the ventral tegmental area are only slightly affected. ${ }^{8}$ In addition, multiple non-DA brain regions show pathology, including the locus coeruleus (noradrenergic), raphe nucleus (serotinergic) and dorsal motor nuclei of the vagus (cholinergic). ${ }^{9-11}$ Non-motor symptoms of PD are typically attributed to degeneration of these brain regions. Notably, it is the key clinical feature of DA loss that forms the basis of the primary symptomatic PD treatment, that being the administration of the DA precursor, levodopa (L-DOPA). ${ }^{12}$ L-DOPA acts to load more DA into the synaptic vesicles within the remaining dopaminergic terminals, thus partially compensating for the loss of innervation. ${ }^{13}$ Unfortunately, dopaminergic PD therapy is typically only effective for three to five years, before the onset of its characteristic side effect, dyskinesias (uncontrolled movements). After five to ten years of treatment, the dose required to control the symptoms of PD approaches that which induces dyskinesias. While L-DOPA is initially effective in addressing the motor features of the disease, other medications are often required to address the non-motor symptoms. Importantly, there is no current clinical therapy available that has been shown to slow or reverse PD.

Beyond neuronal loss, a key histologic feature of PD is the Lewy body (LB), a cytoplasmic inclusion body, found within neurons of the affected brain regions. ${ }^{14}$ LBs are 
common to almost all subsets of PD patients upon autopsy at death. It is however worth nothing that LBs are not exclusive to the disease, as they have also been found in AD patients and healthy people of extreme age. ${ }^{15,16}$ That said, Braak et al has proposed a clinical staging of sporadic PD neurodegeneration based on LB migration through the brain. In Braak Stage 1, LBs are first found in the enteric nervous system, dorsal motor nuclei and olfactory bulb, a neuropathology corresponding both to subtle autonomic dysfunction and to the loss of smell often found to precede motor symptom of PD by years. ${ }^{17-21}$ In Stage 2, LBs are found within the pons and medulla, associated with further disruption of autonomic function. In stage 3, motor symptoms are first evident, as the SNc, amygdala and hippocampus show pathology. In the final stages of 4 and 5, cortical areas are affected and cognitive process deterioration and dementia result. ${ }^{22}$ Such a staging system is suggestive of a systemic pathology, whereby the PD- associated degeneration of the $\mathrm{SN}$ is a relatively late-stage phenomenon. Although a definitive pathogenic mechanism is lacking, several recent papers are supportive of a theory of systemic migration of LB-pathology. In particular, brain autopsies completed upon the death of PD patients, who years earlier received a fetal neuron graft to the SN, revealed LB localization within neurons of fetal origin. ${ }^{23,24}$ Subsequently collected in vitro data indicates that neurons can endocytose protein aggregate structures, allowing for neuron to neuron transport and induction of protein aggregation. ${ }^{25-27}$ Moreover, a recent paper published by Pan-Montojo et al revealed that mouse intragastric administration of rotenone, a pesticide inhibiting the Complex I of the mitochondrial electron transport chain (ETC, Complexes IIV), induced protein aggregate structures within the enteric nervous system. ${ }^{28}$ Such a staging mechanism suggests a primacy of the LB to PD pathology. 


\section{INTRODUCTION}

Table II-1. PD-causing genes and susceptibility factors.

\begin{tabular}{|c|c|c|c|c|c|}
\hline Park loci & Gene/protein & Forms of PD & Putative function & $\begin{array}{l}\text { Association } \\
\text { with UPS }\end{array}$ & $\begin{array}{l}\text { Presence } \\
\text { in LBs }\end{array}$ \\
\hline PARK1/4 & $S N C A / \alpha-S y n u c l e i n$ & $\begin{array}{l}\text { Early-onset autosomal } \\
\text { dominant and sporadic } \\
\text { PD }\end{array}$ & $\begin{array}{l}\text { Synaptic vesicle } \\
\text { function }\end{array}$ & Yes & Yes \\
\hline$P A R K 2$ & Parkin/Parkin & $\begin{array}{l}\text { Juvenile and early- } \\
\text { onset autosomal } \\
\text { recessive and sporadic } \\
\text { PD }\end{array}$ & E3 ubiquitin ligase & Yes & Yes \\
\hline PARK5 & $U C H-L 1 / \mathrm{UCH}-\mathrm{L} 1$ & $\begin{array}{l}\text { Late-onset autosomal } \\
\text { dominant }\end{array}$ & $\begin{array}{l}\text { Ubiquitin C- } \\
\text { terminal hydrolase }\end{array}$ & Yes & Yes \\
\hline PARK6 & Pink-1/PINK-1 & Autosomal recessive & $\begin{array}{l}\text { Protein kinase, } \\
\text { mitochondrial } \\
\text { function }\end{array}$ & Putative & Yes \\
\hline PARK7 & $D J-1 / \mathrm{DJ}-1$ & $\begin{array}{l}\text { Early-onset autosomal } \\
\text { recessive }\end{array}$ & $\begin{array}{l}\text { Oxidative stress, } \\
\text { chaperone }\end{array}$ & Putative & $\begin{array}{l}\text { Yes, in } \\
\text { minority }\end{array}$ \\
\hline PARK 8 & $L R R K 2 /$ dardarin & $\begin{array}{l}\text { Late-onset and sporadic } \\
\text { PD }\end{array}$ & $\begin{array}{l}\text { GTPase, protein } \\
\text { kinase }\end{array}$ & Putative & $\begin{array}{l}\text { Small } \\
\text { minority }\end{array}$ \\
\hline PARK9 & ATP13A2/ATP13A2 & $\begin{array}{l}\text { Juvenile autosomal } \\
\text { recessive (Kufor- } \\
\text { Rakeb syndrome) and } \\
\text { early-onset PD }\end{array}$ & $\begin{array}{l}\text { Lysosomal type } 5 \\
\text { P-type ATPase }\end{array}$ & Putative & $\begin{array}{l}\text { Yes, in } \\
\text { minority }\end{array}$ \\
\hline$P A R K 13$ & OMI/HTRA2/HTRA2 & Sporadic PD & $\begin{array}{l}\text { Mitochondrial } \\
\text { serine protease }\end{array}$ & $?$ & $\begin{array}{l}\text { In small } \\
\text { minority }\end{array}$ \\
\hline $\begin{array}{l}\text { PD } \\
\text { susceptibility } \\
\text { gene }\end{array}$ & $G B A / G B A$ & $\begin{array}{l}\text { Unclear, recessive for } \\
\text { GD }\end{array}$ & $\begin{array}{l}\text { Associated with } \\
\text { lysosomal function }\end{array}$ & $?$ & $?$ \\
\hline
\end{tabular}

Abbreviations: GD, Gaucher's disease. Gene definitions: ATP13A2, lysosomal P-type transmembrane cationtransporting ATPase; GBA, glucocerebrosidase; PINK1; PTEN-induced putative kinase1; LRRK2, leucine-rich repeat kinase 2; Omi/HTRA2, HTRA serine peptidase2; $U C H$-L1; ubiquitin C-terminal hydrolase-L1.

Table adapted from: Bandopadhyay R et al., Pathogenesis of Parkinson's disease: emerging role of molecular chaperones. Trends in Molecular Medicine. 2009; 16: 27-36.

\subsubsection{Idiopathic and familial forms of PD}

Ninety percent of diagnosed PD cases are idiopathic, that being, arising from an indefinable cause. However the remaining $5-10 \%$ of cases are attributable to familial forms of PD. Both recessive and dominant Mendelian inheritance patterns of PD have been identified. Linkage data has to date revealed ten PARK loci (Table II-1). ${ }^{29}$ The important PD-linked loci are summarized in Table II-1. For seven loci, causative genes have been identified: three 
autosomal dominant (SNCA (PARK1/4, $\alpha$-Synuclein), leucine rich repeated kinase (LRRK2, PARK8) and UCH-L1 (PARK5)) and four autosomal recessive (Parkin (PARK2), PTENinduced putative kinase 1 (PINK1, PARKO), DJ-1 (PARK7) and ATP13-A2 (PARK9)). It should be noted that the importance of UCHLI in broader PD has yet to be established. ${ }^{12} \mathrm{~A}$ susceptibility allele $(H T R A 2 / O M I)$ at the PARK13 locus has been identified, although recent data questions whether this is a strongly- associated risk factor in world- wide PD populations. ${ }^{30}$ Although genetic PD represents only a small percentage of patients, mutations in these genes have also been identified in sporadic PD. This suggests that the mechanisms of disease and cell death of genetic PD can be extrapolated to the idiopathic form.

A brief introduction to the genes relevant to this project, that being SNCA, Parkin, PINK1 and DJ-1, follows, with particular emphasis on SNCA. A more detailed discussion of how each gene might fit into theories of overall disease pathogenesis follows in the next section.

\subsubsection{1. $\quad \alpha$-Synuclein (SNCA, PARK1/4)}

$S N C A$ was the first identified PD gene. Previous to its association with the disease, $\alpha-$ Synuclein had been identified as a protein localized to presynaptic terminals of the electric ray and rat brain. ${ }^{31}$ A human homologue was identified in 1993 and found to be a component of amyloid plaques in AD. ${ }^{32}$ In 1995, a $\alpha$-Synuclein homologue, Synelfin, was linked to synaptic plasticity involved in song bird learning. ${ }^{33}$ However it was not until 1997 when two papers were published, the first describing a dominantly inherited familial case of PD resulting from an [A53T] SNCA mutation and the second showing that $\alpha$-Synuclein was the major protein component of the LB, that $\alpha$-Synuclein was definitively linked to PD. ${ }^{34,35}$

Two additional missense mutations of SNCA ([A30P] and $[\mathrm{E} 46 \mathrm{~K}])$ resulting in PD have been since described. ${ }^{36,37}$ These mutations are quite rare, as [A53T] has only been found in families of Greek origin, [A30P] in one German family and [E46K] in a Spanish family. 
The $[$ A53T] mutation results in an early onset (mean forty-five years) and rapid disease progression. People affected by the $[\mathrm{A} 30 \mathrm{P}]$ and $[\mathrm{E} 46 \mathrm{~K}]$ mutation generally show a typical PD disease progression. Multiple instances of duplications and triplications of the gene have been identified. Duplications are often indistinguishable from idiopathic PD, while triplications result in early onset, rapid disease progression and reduced lifespan. ${ }^{38-42}$ Duplication of SNCA has been identified in several sporadic PD cases. ${ }^{43}$ In addition, a polymorphism in the $S N C A$ promoter region that in vitro, upregulates expression, has been identified as a risk factor for idiopathic PD. ${ }^{44}$ In total, the inheritance patterns suggest that there is gain of function with SNCA mutation and indicate a critical effect of protein dose.

Human $\alpha$-Synuclein is a small protein that is natively unfolded in aqueous solution. Important to hypotheses relating to $\alpha$-Synuclein pathology is its ability to change conformation and take on more complex three- dimensional structures in response to alteration of its chemical environment. ${ }^{1}$ Three major domains have been delineated: an aminoterminal repeat domain, a non-amyloid component (NAC) domain and an acidic carboxylterminal domain. The highly conserved amino repeat domain is key to the protein's transformation into an amphipathic $\alpha$-helical structure, an alteration enabling membrane binding. ${ }^{45}$ All three of the PD missense mutations are found within the $\mathrm{N}$ terminus repeat domain. In addition, the $\mathrm{N}$ terminus domain has been found to contain a cryptic mitochondrial targeting sequence. ${ }^{46}$ The NAC domain is named on the basis of its sequence similarity to the NAC peptide of Alzheimer's amyloid fibrils. Its hydrophobicity allows a transformation from unfolded native protein into soluble protofibrils, oligomers and insoluble fibrils (see Fig.II1). ${ }^{47,48}$ The C-terminus hydrophilic domain contains several phosphorylation sites that have been implicated as important to both normal $\alpha$-Synuclein function and its pathogenic actions. $^{49-52}$ 
Despite widespread research interest in $\alpha$-Synuclein, a clear physiologic function for the protein has yet to be determined. Under physiologic conditions, the majority of $\alpha$ Synuclein is localized to the presynapse. At the same time, the protein is able to bind PL and can bind polyunsaturated fatty acids. These properties are suggestive of a function related to vesicle or fatty acid metabolism. Indeed, lipid abnormalities have been noted in the brains of SNCA knockout (KO) mice. ${ }^{53-58}$ Most recent data suggests a positive role for $\alpha$-Synuclein in both the formation of synaptic vesicles and for neurotransmitter release. Neurons lacking the protein show reduced numbers of synaptic boutons, perhaps indicating decreased synaptic activity. ${ }^{59}$ However, as defects in neurotransmission in SNCA KO are subtle, it appears $\alpha$ Synuclein is a non-essential protein for these processes. Finally, multiple studies have implicated a role for $\alpha$-Synuclein in endoplasmic reticulum (ER)-Golgi membrane mechanics. In particular, several papers reported that the wild type (wt) protein could inhibit ER-Golgi vesicle docking, a function rescued by Rab1 overexpression, a GTPase (guanosine triphosphate) that regulates ER-derived vesicles transport. ${ }^{60,61}$ In sum, a role for physiologic $\alpha$-Synuclein in membrane mechanics is implicated.

$\alpha$-Synuclein may act as a molecular chaperone under certain conditions. Cysteine string protein- $\alpha(\mathrm{CSP}-\alpha)$ is a co-chaperone of the SNARE (SNAP (Soluble NSF Attachment Protein) Receptors) complex required for synaptic vesicle exo- and endocytosis. Chandra et al demonstrated that expression of $\alpha$-Synuclein is able to ameliorate neurodegeneration that results from deletion of CSP- $\alpha$ in mice, an amelioration in part due to partial restoration of the SNARE complex. ${ }^{62}$ Several other papers, conducted in worm (C. elegens), neuroblastoma and primary neuron culture, additionally posit a role for $\alpha$-Synuclein in neuronal survival, as low level expression is protective against oxidative stress-induced apoptosis and excitotoxicity. ${ }^{63-}$ 66 Thus the specific mechanisms and conditions by which $\alpha$-Synuclein might be neuroprotective have yet to be clearly delineated. 
Perhaps related to its putative chaperone function, $\alpha$-Synuclein has also been linked to DA metabolism. $\alpha$-Synuclein shares $40 \%$ sequence homology with the molecular chaperone 14-3-3. ${ }^{67}$ Both 14-3-3 and $\alpha-$ Synuclein can bind tyrosine hydroxylase $(\mathrm{TH})$, the rate limiting enzyme of DA synthesis. ${ }^{68} \alpha$-Synuclein can also complex with the DA transporter (DAT), a binding that could alter protein localization and thereby affect DA reuptake from the synapse. ${ }^{69}$ Thus, a possible role for $\alpha$-Synuclein in the regulation of DA homeostasis is implicated.

Finally, recent findings indicate that $\alpha$-Synuclein plays a physiologic role within the mitochondria. Several papers have demonstrated that $\alpha$-Synuclein can enter mitochondria and is associated with the inner membrane. Once inside, $\alpha$-Synuclein has been shown to inhibit Complex I activity. However, Complex I inhibition may not to be correlated to $\alpha$-Synuclein pathology, as evaluated in terms of protein aggregation. ${ }^{70}$ At the same time, $\alpha$-Synuclein KO mice show alterations to mitochondrial membrane composition linked to decreased activity of ETC components. ${ }^{71} \mathrm{KO}$ mice also show resistance to mitochondrial toxins, suggesting that $\alpha$ Synuclein may moderate the mitochondrial response to oxidative stress. ${ }^{72}$ A small amount of reactive oxygen species (ROS) are naturally produced as electrons move through the ETC in the mitochondrial inner membrane as part of oxidative phosphorylation production of ATP. Oxidative stress refers to a condition in which there is an excess of ROS (oxygen ions, free radicals and peroxides) due to either inadequate antioxidant capacity or by overproduction. These results thus suggest several levels of mitochondrial dysfunction in the context of $\alpha$ Synuclein.

Thus, the extent to which mutation or overexpression of $\alpha$-Synuclein negatively affects normal physiologic function or alternatively affects new processes due to gain of function remains a topic under investigation.

\subsubsection{Parkin (PARK2)}


The PARK2 locus is associated with an autosomal recessive, early onset (less than 50 years) form of PD. Parkin mutations were first identified in Japanese family and have since been found in multiple families of different ethnic background. Over one hundred Parkin mutations have been identified, including point mutations, deletions and exon multiplications, making it the second most common mutation of PD. In total, $50 \%$ of familial recessive early onset (less than 45 years) and $77 \%$ of sporadic PD with onset before age 20 are attributable to Parkin mutations. ${ }^{73,74}$ The prevalence of Parkin mutations suggests an important role for the protein in suppressing PD. ${ }^{75}$ Patients with homozygous Parkin mutations demonstrate typical PD symptoms, however show an earlier age of onset, though with slower disease progression. Heterozygous mutations have also been found in instances of idiopathic PD. ${ }^{76}$ An atypical pathohistology is found in Parkin-related PD, that being a general absence of LBs within the brain. $^{77}$

Ubiquitously expressed throughout the body, Parkin is a ubiquitin E3 ligase and functions as part of the ubiquitin proteasome system (UPS). ${ }^{78,79}$ The purpose of the UPS and heat shock chaperone proteins is to clear misfolded proteins from the cell. Damaged proteins tagged with multiple ubiquitins (poly-ubiquitylation) are sent to the $26 \mathrm{~S}$ proteasome for breakdown. E3 ligases such as Parkin function essentially as gatekeepers to the proteasome, as they are the substrate-recognition molecules used for the attachment of polyubiquitin chains. ${ }^{12,80}$ Accumulation of Parkin substrates CDCEL1 and PAELR has been found within the brains of patients with Parkin-related PD, suggesting a direct involvement of the UPS system in PD pathology. ${ }^{81,82}$ Of note, in vitro studies have shown Parkin able to ubiquitinate three other PD-associated genes: mutant DJ-1 (PARK7), glycosylated $\alpha$-Synuclein and Synphilin, a binding partner of $\alpha$-Synuclein. ${ }^{83,}{ }^{84}$ Thus, Parkin may be critical to the recognition of potentially toxic proteins in neurons. Parkin-DJ-1 interactions have been heavily investigated. Interestingly, ubiquitylation of DJ-1 does not lead to its degradation by 
the proteasome, but rather to its transport to an inclusion body known as the aggresome. Toxic proteins placed in the aggresome can be cleared via autophagic mechanisms, suggesting Parkin may additionally function to remove toxic proteins via protective aggresomal accumulation. ${ }^{84-86}$ It moreover implicates possible derangement of autophagy, an intracellular bulk degradation system, as playing a role in PD pathology.

Parkin also has a mitochondrial function. $\mathrm{KO}$ of Parkin in the fruit fly results in a severe mitochondrial phenotype, typified by swollen mitochondria, oxidative stress, alteration to ETC components and eventual death of the flight muscles. ${ }^{87}$ Although not as severe a mitochondrial phenotype as found in flies, Parkin $\mathrm{KO}$ in mice also enhances oxidative stress and reduces expression of multiple ETC proteins. ${ }^{75}$ Most recently, data indicates Parkin ubiquitylation of dysfunctional mitochondria targets these organelles for mitophagy, through a mechanism similar to that of protein clearance. ${ }^{88,89}$ Thus, dysfunction in Parkin-related PD seems most likely to result from defects in critical cell functions of UPS and mitochondrial maintenance.

\subsubsection{PINK1 (PARKG)}

Similar to the PARK2 locus, the PARK6 locus is associated with autosomal recessive PD, with both early (age 32-48) and late onset. The disease-causing gene was identified as PINK1 and was first found in three Italian-Spanish families. Multiple missense and nonsense of the protein have been recognized. ${ }^{90}$ Like Parkin, heterozygous mutations of PINK1 have been identified in cases of sporadic PD. ${ }^{91}$ It is the second most common form of autosomal recessive PD after Parkin. ${ }^{75,92}$

PINK1 is a ubiquitously expressed, highly conserved serine-threonine kinase. It was first identified as a substrate of PTEN, an oncogene mutated in many cancer cells. ${ }^{93}$ Via its Nterminal mitochondrial targeting motif, PINK1 localizes to the mitochondrial matrix and intermembrane space. The protein is important to mitochondrial function and in particular, is 
neuroprotective against oxidative stress-induced apoptosis. Overexpression of wild type PINK1 is protective against mitochondrial toxins such as the Complex I inhibitor MPTP, while siRNA in cell culture systems results in an increased susceptibility to apoptosis. Protein overexpression is also associated with a higher mitochondrial membrane potential and a decrease in Cytochrome c release. ${ }^{77}$ 94, 95 As a physiologic correlate to cell culture data, fibroblasts and lymphoblasts from PD patients with PINK1 mutations show decreased Complex I activity and increased levels of oxidative stress. ${ }^{91,96}$ Obvious mitochondrial pathology is found with Drosophila PINK1 KO, including misformed mitochondrial structure, increased sensitivity to oxidative stress, locomotor defects and decreased lifespan. ${ }^{97-100}$ Similarly, mouse KO of PINK1 and human DA neurons treated with siRNA for PINK1 show a multitude of mitochondrial deficits that are similar to the defects seen in non-human neuronal cell culture. These deficits include reduced membrane potential, altered morphology, increased ROS production, impaired Complex I activity and sensitivity to oxidative stress. ${ }^{101 \text {, }}$ 102

The mechanism by which PINK1 is protective of mitochondrial function has only recently begun to be elucidated. In 2007 Pridgeon et al reported the first known substrate of PINK1 to be TRAP1, an HSP90-like mitochondrial chaperone protein. In cell culture, PINK1 is protective against oxidative stress- induced cell death via inhibition of Cytochrome c release. This inhibition is dependent on its ability to phosphorylate TRAP1. Familial mutations of PINK1 inhibit TRAP1phosphorylation. ${ }^{103}$ TRAP1 has been noted in several cell systems, including multiple types of carcinoma, hepatocytes, and cardiomyocytes, to be protective against ischemic injury, ROS and apoptosis. ${ }^{104-106}$ In addition, a basic worm $(C$. elegens) genetic screen for suppressors of $\alpha$-Synuclein aggregation noted that use of RNAi against the R151.7 (worm homologue of human TRAP1) enhanced aggregation. ${ }^{107}$ Thus, the 
PINK1 protective effect may be induced via activation of conserved and essential defense against mitochondrial toxicity.

A second identified PINK1 substrate is the mitochondrial serine protease, HTRA2/Omi. After PINK1 phosphorylation at serine 142, HTRA2 is thought to mediate stressed neuron survival. This theory is supported by HTRA2 $\mathrm{KO}$ mice who display striatal neurodegeneration and early death. Also worth noting is that while brains from sporadic PD patients show widespread serine 142 phosphorylation, PINK1 PD patients show relative absence of phosphorylation, suggestive of deficient HTRA2 phosphorylation. ${ }^{108}$ Most interestingly, Parkin has been shown to act downstream of PINK1. This was first suggested due to the similarity of Drosophila phenotypes resulting from either KO of Parkin or PINK1. Indeed, while PINK1 overexpression was unable to rescue KO of Parkin, the reverse, that being Parkin overexpression and PINK1 KO, was true. ${ }^{98-100}$ Most recent data suggests a mechanism whereby PINK1 is selectively stabilized and its kinase activity upregulated in mitochondria damaged by oxidative stress. The protein is then able to recruit Parkin to damaged mitochondria. Parkin-mediated ubiquitylation then targets these mitochondria for autophagy. ${ }^{109,} 110$ Thus, PINK1 plays multiple protective roles within the mitochondria, resulting in a loss of function phenotype with $\mathrm{PD}$-associated mutation.

\subsubsection{DJ-1 (PARK7)}

$D J-1$ mutations cause autosomal recessive PD. Mutations in $D J-1$ are very rare (1\% of PD mutations). Only a deletion mutant and several point mutations, one of which causes early-onset PD, have been identified..$^{92,111}$ Found in both neurons and glia, DJ-1 is a highly conserved, multifunctional and ubiquitously expressed protein. It appears to have both antioxidant and transcription modulatory activity. ${ }^{112}$ In cell culture, DJ-1 is typically found in the cytosol. However upon oxidative stress inducement, DJ-1 moves into the mitochondria. Translocation into the mitochondria correlates to its neuroprotective effect. ${ }^{113} \mathrm{KO}$ animal 
models further support the hypothesis of DJ-1 acting as a mitochondrial protective factor. Drosophila DJ-1 KO produces animals with increased sensitivity to oxidative stress and the mitochondrial specific pesticide, paraquat. Degeneration of DA neurons is also observed. ${ }^{100,}$ 114 DJ-1 KO mice show multiple striatal- alterations, including increased DA reuptake, elevated striatal DA levels and hypokinesia. An enhanced sensitivity to MPTP or paraquat has been noted, manifesting itself as increased DA cell death. ${ }^{115}$ As DJ-1 is protective against Complex I inhibitors, it may directly protect Complex I from oxidative stress-induced damage.

The overall neuroprotective mechanism of DJ-1 has yet to be fully elucidated. However DJ-1 inclusion in a common pathway of oxidative stress protection is suggested by data showing that DJ-1 can interact with both Parkin and PINK1. In vitro, DJ-1 can increase over-expressed PINK1 steady state levels. Under oxidative stress conditions, Parkin has been shown to bind DJ-1. ${ }^{84,116}$ One paper reported the in vitro formation of a DJ-1, PINK1 and Parkin complex, which promoted ubiquitylation of Parkin substrates. ${ }^{117}$ Additional evidence of a common neuroprotective pathway that is abrogated by loss of function mutation is suggested by a family with digenic mutation in both PINK1 and DJ-1 showing early onset PD. ${ }^{118}$

\subsection{PD etiology and pathogenesis}

The next section will describe the several overlapping theories that provide a framework for understanding the mechanism of PD neurodegeneration, with particular emphasis on $\alpha$-Synuclein toxicity.

\subsubsection{UPS dysfunction and protein aggregation}

The phenomenon of protein misfolding and aggregation is common to many neurodegenerative disorders, including $\mathrm{PD}, \mathrm{AD}$ and Huntington's disease (HD). As mentioned, a PD pathologic hallmark is the LB, with the major protein component being 
amyloid fibrils of $\alpha$-Synuclein. Presence of $\alpha$-Synuclein in the LB would not be sufficient to indicate a causal role of protein misfolding in PD. However, $\alpha$-Synuclein mutations are sufficient to induce autosomal dominant PD. At the same time, Parkin and UCH-L1 (a ubiquitin C-terminal ligase) are both involved in the UPS system of protein degradation and mutations in both are causal in recessive PD. ${ }^{119}$ Together, these data suggest protein aggregation is a key step in PD pathogenesis.

As shown in Fig.II-1, $\alpha$-Synuclein can take on several different forms: a membrane bound alpha-helix, the natively unfolded monomer, $\beta$-sheet oligomers and the insoluble fibrils found in LBs. ${ }^{120}$ Mutated $\alpha$-Synuclein (familial mutations [A53T], [A30P] and [E46K]) has a heightened propensity to aggregate. [A53T] and [E46K] assume fibrillar structures more rapidly than wt Synuclein, while [A30P] fibrillizes more slowly. ${ }^{121-123}$ At the same time, [A30P] and [A53T] can form intermediate oligomer structures faster than wt. The [E46K] mutant does not assume this structure as readily. ${ }^{122,}{ }^{124}$ Phosphorylation of the protein has been shown important to structure. In particular, $\alpha$-Synuclein found in LB and aggregates is typically phosphorylated at S129, although the toxic significance of this modification seems dependent on experimental system used..$^{51,125-127}$ At the same time, $\alpha$-Synuclein mutation or overexpression results in cytotoxicity, with [A53T] being the most toxic variant. Direct cell loss is induced both in vitro and in the in vivo models of yeast, Drosophila, C. elegens, rat, mouse and non-human primate. ${ }^{128-137}$ The question of whether the insoluble LB aggregate itself is toxic or is in fact protective remains contested. In particular, studies have noted that certain $\alpha$-Synucleins may show enhanced cytotoxicity without increasing insoluble aggregative species. ${ }^{138}$ Additional experiments have suggested that the oligomeric species, a species which [A53T] and [A30P] more readily form, may be the toxic species of $\alpha$ Synuclein. ${ }^{45,139,140}$ In particular, Karpinar et al demonstrated that variants of $\alpha$-Synuclein with the greatest tendency to form soluble oligomers show the greatest neurotoxicity both in 
vitro and in vivo (Drosophila and C. elegans). Of the natural mutants tested, [A53T] generally resulted in the most severe phenotype. ${ }^{141}$ At the same time, expression of the molecular chaperone, HSP70, is able to suppress toxicity caused by overexpression of wt, [A53T] or

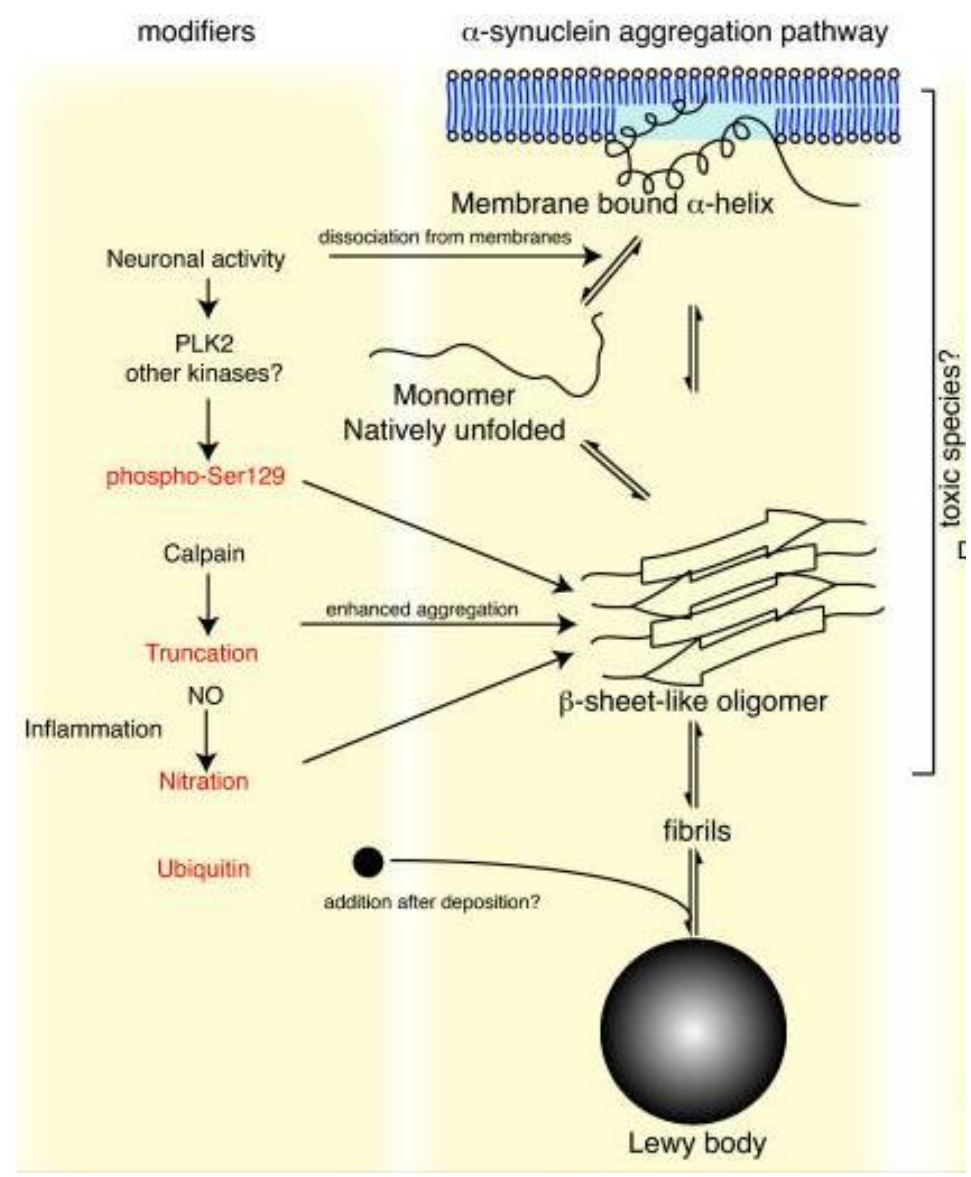

Fig.II-1. Different 3D structures of $\alpha$ - Synuclein. $\alpha$-Synuclein can transition from its native unfolded state to an intermediate oligomer structure, followed by an insoluble fibrillar structure. The fibrillar form is that found in the LB. The protein can also interact with membranes via transition into an alpha-helical structure. Various factors have been associated with $\alpha$-Synuclein's conversion between different structures. For example, phosphorylation state (at S129) and nitration both promote fibrillization. Fibrillar $\alpha$-Synuclein found in LB is typically ubiquitinated.

Adapted from: Cookson MR. Synuclein and neuronal cell death. Molecular Neurodegeneration. 2009; 4:9.

[A30P] $\alpha$-Synuclein in Drosophila. Toxicity suppression occurred without a decrease in aggregated $\alpha$-Synuclein, indicating that either monomeric $\alpha$-Synuclein by itself is toxic or that inhibition of formation of downstream intermediate species was sufficient to reduce toxicity. 142 The mechanism whereby $\alpha$-Synuclein oligomeric species might cause increased toxicity has yet to be resolved. $\alpha$-Synuclein oligomers show a capacity to form annular or pore-like structures. One paper provided data indicating that oligomeric $\alpha$-Synuclein triggered calcium 
entry, with subsequent increased toxicity in human neuroblastoma SH-SY5Y cells, while another demonstrated that cells expressing $\alpha$-Synuclein show an increased cation permeability. ${ }^{140,} 143$ Thus, it is possible that $\alpha$-Synuclein oligomers may act to damage intracellular membranes or synaptic vesicles. Definitive data is at present lacking.

An additional aspect of protein aggregation is $\alpha$-Synuclein interference with the lysosomal system. Monomeric $\alpha$-Synuclein can be broken down within lysosomes and oligomers of two $\alpha$-Synuclein molecules can also undergo degradation via an alternate lysosomal pathway, chaperone-mediated autophagy (CMA). Oligomers of greater than two $\alpha-$ Synucleins are resistant to CMA and can accumulate on lysosomal membranes, acting to block the process. Interestingly, high levels of wt $\alpha$-Synuclein or [A53T]/[A30P] mutants, inhibit uptake of other CMA protein substrates, resulting in substrate accumulation. ${ }^{144}{ }^{145}$ The end result of this blockade may be cell death via impaired degradation of myocyte enhancer factor 2D (MEF2D), a neuronal survival transcription factor. Decreased degradation of MEF2D results in less efficacious function, threatening neuronal survival. ${ }^{146}$

A final feature of protein degradation worthy of note is dysfunction in the UPS system. Rodents exposed to proteasomal inhibitors develop symptoms that closely recapitulate PD. ${ }^{147}$ Moreover, loss of function mutations in UPS system Parkin and UCHL1 result in PD. Disturbance of the UPS can directly lead to cell death. ${ }^{148} \alpha$-Synuclein may itself play a role in proteasomal dysfunction, as multiple papers have evidenced firstly that it is a substrate of the system (mono and bi-ubiquitin) and secondly, in vitro, $\alpha$-Synuclein can induce proteasomal dysfunction. ${ }^{149-152}$ Although it is known that $\alpha$-Synuclein protofibrils can inhibit the main site of UPS protein degradation, that being the $26 \mathrm{~S}$ proteasome ${ }^{153}$, the mechanisms by which $\alpha$-Synuclein interferes with the proteasomal system are poorly described. An additional point of interest is the observation that in early onset Parkin-related PD, LBs are not typically found. This suggests that Parkin-ubiquitylation of $\alpha$-Synuclein may 
be required for the formation of large $\alpha$-Synuclein aggregates. ${ }^{154}$ Thus, there may be a convergence of PD related genes in a common pathway related to $\alpha$-Synuclein aggregation and removal.

\subsubsection{Environmental toxins}

On the basis of several drug-induced models of PD, environmental neurotoxins have been implicated in the development of sporadic PD. In particular, the pyridine analogue and synthetic heroin derivative MPTP is selectively taken up into DA neurons. It is then metabolized into MPP+, which acts to inhibit mitochondrial ETC Complexes I, III and IV. ROS release and ATP depletion ultimately results in the selective death of DA neurons and a PD-like motor disorder syndrome. Experimentally, both acute and chronic treatment of MPTP to animals results in a PD-like syndrome. Notably however, only chronic administration results in LB-like aggregates. Similarly, treatment with the commonly used pesticide rotenone, a Complex I inhibitor, reproduces many of the features of PD including DA cell loss. Toxic effects of low dose administration include LB-like formation containing $\alpha$ Synuclein, pronounced ROS production causing damage to multiple cellular components, alongside mitochondrial degeneration. ${ }^{28,}{ }^{155}$ Paraquat is a herbicide with similar chemical structure to MPTP. Administration of the compound to experimental animals again results in a PD-like syndrome, with DA cell degeneration and motor deficits. In vitro tests show that paraquat interacts with the DAT and results in weak Complex I inhibition alongside marked ROS production and antioxidant depletion. ${ }^{156}$ Lifetime exposure to such compounds has thus been linked to the development of sporadic PD. ${ }^{157,158}$ A Costello et al study demonstrated that high level combined exposure to both paraquat and another pesticide, maneb, when patients were under age 60, increased the risk of developing PD by $75 \% .^{159}$ Similarly, trichloroethylene, a compound used as a degreaser for military and industrial applications, is a Complex I inhibitor and also a risk factor for PD. ${ }^{160}$ Importantly, a reduction in Complex I 
activity in the brains of idiopathic PD patients has been described, evidence to the importance of mitochondrial dysfunction to PD pathogenesis, while also implicating a role for Complex I inhibitors in causing/exacerbating idiopathic PD. ${ }^{161,162}$ Thus, the drug induced-mechanisms of PD are suggestive of a mitochondrial- based PD pathogenesis, one linked to ETC dysfunction, ROS overproduction and ATP depletion.

\subsubsection{Mitochondrial dysfunction and oxidative stress}

In addition to data evidencing a role for mitochondrial toxins directly interfering with ETC components, aging-associated oxidative stress and ROS overproduction have been theorized to be contributing factors in mitochondrial dysfunction. In aged patients, up to one third of cellular proteins show oxidative modification. Accumulation most likely results from ROS over production, alongside both decreased antioxidant activity and reduced ability to remove altered proteins. ${ }^{163-165}$ Importantly, within the SN of sporadic PD patients, several Complex I catalytic units have been found to carry excess carbonyls, an oxidative modification. This modification correlated to reduced rates of electron transfer and may indicate oxidative damage leading to the noted Complex I dysfunction found in PD brains. ${ }^{166}$ The role of oxidative stress and mitochondrial dysfunction as a causal in PD relates to a possible reason for the particular vulnerability of DA neurons to cell death. That is, a large body of evidence suggests DA metabolism itself resulting in an excess of ROS production that could lead to additional mitochondrial damage. Typically DA is sequestered within acidic vesicles, where low $\mathrm{pH}$ prevents oxidation. However, if DA remains in the cytosol, it can oxidize to produce multiple byproducts, including superoxide, hydrogen peroxide and DA quinones. In vitro and in vivo rodent experiments have shown that excessive cathecholamine oxidation can result in neuronal death. ${ }^{165}$ Interestingly, in vitro data indicates mutant $\alpha$ Synuclein can impair vesicular storage of DA, allowing its oxidation. ${ }^{167}$ At the same time, oxidized DA can promote the conversion of $\alpha$-Synuclein to oligomeric or fibrillar forms. ${ }^{168 \text {, }}$ 


\section{INTRODUCTION}

${ }^{169}$ In addition, neuromelanin, a product of DA oxidation, is present in SN DA neurons and binds to iron, which can lead to free radical production. ${ }^{170}$ A further point suggestive that oxidative stress as being key to DA neuronal loss is that in $\alpha$-Synuclein[A30P] expression or Parkin and PINK1 KO Drosophila models, overexpression of either human $\mathrm{Cu} / \mathrm{Zn}$ super oxide dismutase (SOD) or glutathione- $\mathrm{S}$ transferase can successfully rescue DA neuron loss. ${ }^{171,172}$ That said, it is at present difficult to resolve whether or not the enhanced oxidative stress found in PD patients is the cause of mitochondrial dysfunction or the result.

Indeed, a clear role for impaired mitochondrial function in PD development is suggested by the convergence of data from both genetic and sporadic PD implicating the mitochondria. In particular, KO or mutation of Parkin, PINK1 or DJ-1 leads to protein loss of function and the associated mitochondrial deficits, as above described. A link between sporadic and genetic forms of PD has been made by papers indicating the combined influence of pesticide treatment (paraquat or maneb) and mutant $\alpha$-Synuclein exacerbated PD-like mitochondrial pathology. ${ }^{132}$ Indeed, an additional aspect of the story is $\alpha$-Synuclein 's involvement in the mitochondria. $\alpha$-Synuclein is able to enter the mitochondria via an $\mathrm{N}$ terminus targeting sequence. As mentioned, overexpression or expression of mutated $\alpha$ Synuclein can lead to cell death in both cell and animal models. Mitochondrial dysfunction associated with ATP depletion and ETC dysfunction reduces the cell's ability to handle oxidative protein damage and cellular tasks. At the same time, ETC dysfunction leads to a opening of the mitochondrial PTP, a drop in mitochondrial membrane potential, with subsequent increased Cytochrome c release and death via apoptosis. Indeed, $\alpha$-Synucleinoverexpressing cells exhibit multiple markers of mitochondrial dysfunction, including increased protein oxidation, increased ROS production, loss of mitochondrial membrane potential and reduced Complex I activity. ${ }^{46,70,173-177} \alpha$-Synuclein mutation or overexpression enhances markers of mitochondrial damage. For example, $\alpha$-Synuclein[A53T] in human DA 
neurons resulted in enhanced mitochondrial uptake and reduction in Complex I activity vs. wt protein. Complex I inhibition was dependent on $\alpha$-Synuclein's mitochondrial localization. ${ }^{46}$ While $\alpha$-Synuclein KO mice show resistance to mitochondrial toxins such as MPTP, paraquat and maneb, mice expressing $\alpha$-Synuclein[A53T] demonstrate enhanced sensitivity. ${ }^{46,}$ 175, 176 Importantly, mice carrying a mutation in both Parkin and $\alpha$-Synuclein showed the most severe phenotype, supportive of a multi-hit hypothesis of PD pathogenesis. ${ }^{13,72,134,178,179}$ This data suggests $\alpha$-Synuclein may play a role both in moderating the mitochondrial function and also mediating mitochondrial damage due to toxic gain of function effects upon mutation or increased dose of $\alpha$-Synuclein. The specific determinant of whether or not $\alpha$-Synuclein enters the mitochondria has yet to be determined, although one paper demonstrated enhanced mitochondrial translocation with cytosolic acidification, associated with oxidative or metabolic stress. ${ }^{180}$ However, in vitro findings are buttressed by PD patient histology indicating that there is $\alpha$-Synuclein mitochondrial accumulation isolated to the $\mathrm{SN}$ and striatum. Such accumulation has not been found in control subjects. ${ }^{46}$ Beyond data indicating helical $\alpha$-Synuclein may result in outer mitochondrial membrane permeabilization, with resultant loss of potential, the specific mechanisms by which $\alpha$-Synuclein directly damages the mitochondria are lacking. However what is clear is that the interaction of $\alpha$-Synuclein with the mitochondria is complex, with dysfunction perhaps resulting from both direct $\alpha$ Synuclein interaction and $\alpha$-Synuclein aggregation in the cytoplasm producing oxidative stress, which in turn damages ETC components.

As depicted in Figure II-2, one can thus envision a general schema of mitochondrial damage arising from multiple sources (gain of function mutant $\alpha$-Synuclein, loss of protective proteins PINK1, DJ-1 and Parkin, mitochondrial toxins from the environment) with the end result of cell death. Such a schema integrates factors involved in the development of both sporadic and genetic PD. 


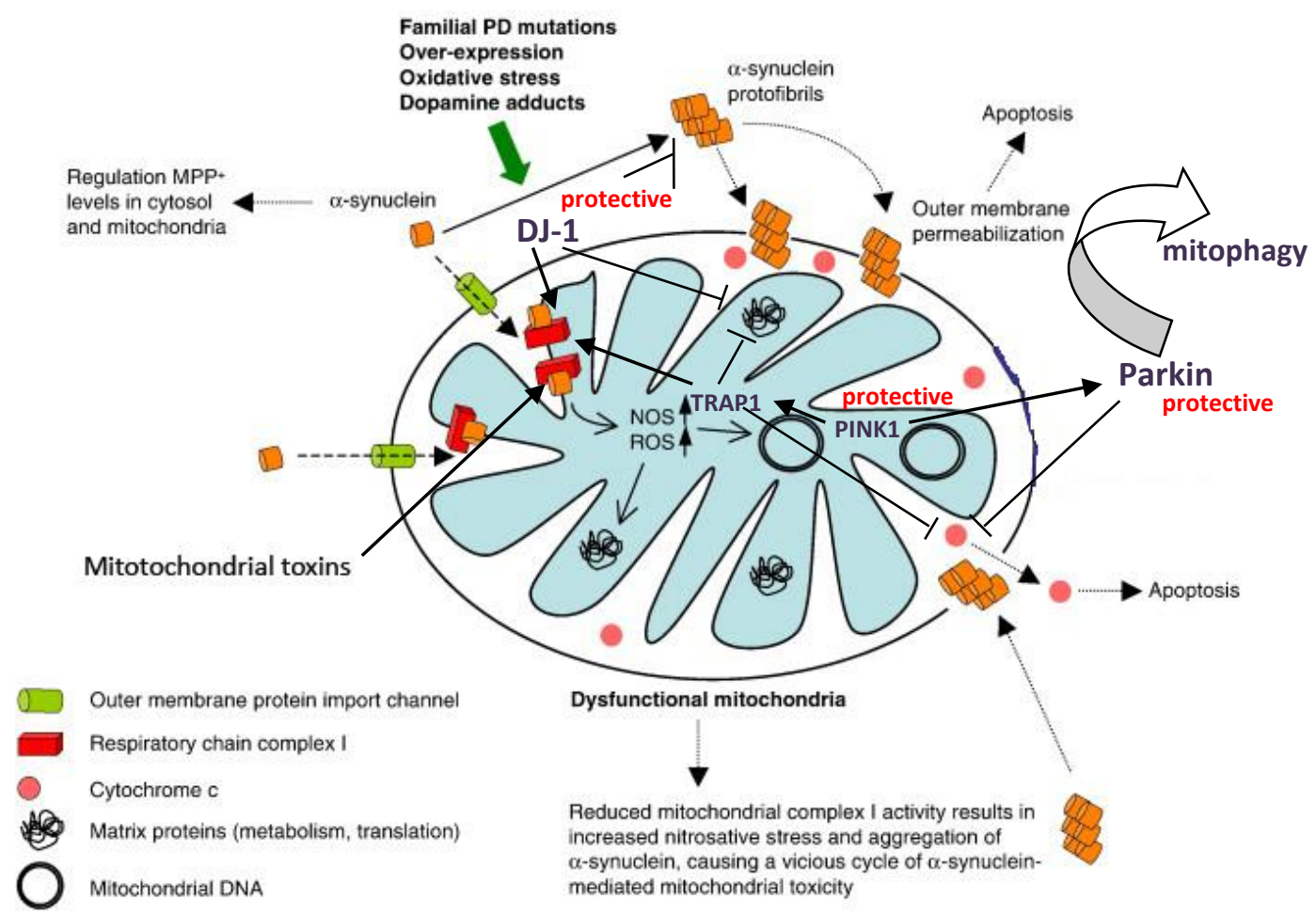

Fig.II-2. Model of mitochondrial dysfunction in PD. $\alpha$-Synuclein can enter the mitochondria, where it can interfere with Complex I activity. Entry is enhanced by cytosolic acidification or $\alpha$-Synuclein mutation. Familial PD mutations such as [A53T], oxidative stress and DA oxidation increase $\alpha$-Synuclein oligomers formation, which may act to permeabilize the mitochondrial membrane, resulting in loss of mitochondrial membrane potential. $\alpha$-Synuclein may also act to regulate MPP+ levels in the cytoplasm. Decreased Complex I activity results in ATP depletion and increased production of nitrative oxygen species and ROS. This in turn might damage mitochondrial DNA and lead to protein aggregation and misfolding within the mitochondria. PINK1 can phosphorylate the mitochondrial chaperone TRAP1. TRAP1 phosphorylation inhibits Cytochrome $\mathrm{c}$ release and may be protective against Complex I dysfunction, ROS production and protein misfolding. Decreased mitochondrial membrane potential causes PINK1 to recruit Parkin to the mitochondria. Parkin has multiple functions including inhibition of Cytochrome $\mathrm{c}$ release and ubiquitylation of damaged mitochondria, targeting them for mitophagy. DJ-1 chaperone activities are regulated by oxidative stress. Loss of DJ-1 enhances sensitivity to oxidative stress and Complex I inhibition, suggesting it is protective of these functions. It also can prevent misfolding of mitochondrial proteins and prevent Synuclein aggregation and toxicity. Thus, loss of PINK1, DJ-1 or Parkin activity through mutation leaves mitochondria both susceptible to injury and with structural defects, while overexpression or mutation of $\alpha$-Synuclein can directly interfere with mitochondrial function. The end result is most likely cell death via apoptotic mechanisms.

Figure adapted from: Bueler H. Impaired mitochondrial dynamics and function in the pathogenesis of Parkinson's disease. Experimental Neurology. 2009; 218: 235-246.

\subsection{Experimental models of PD}

In order to investigate the pathogenesis of $\mathrm{PD}$, multiple experimental models have

been developed. In order to examine the molecular pathways leading to neurodegeneration, the ideal model system would perfectly reproduce all disease features observed in humans, in an age-dependent and heritable manner. Of course, such a perfect system does not exist. Thus, 
the next section will consider the advantages and drawbacks to two models relevant to this project: cell culture and Drosophila melanogaster.

\subsubsection{Cell culture systems}

The use of cell culture provides an advance means to establish molecular pathways, before moving to more complicated, time-involved, expensive and ethically-sensitive animal experiments. In vitro cell culture investigation allows for the obvious advantages of ease of access, controllable external environment, reproducibility and access to sophisticated biochemical and microscopic techniques and manipulations. A wide range of cells have been used to investigate mechanisms of PD.

A cell often used for basic biochemical and protein investigation is the human embryonic kidney-293 (HEK293) cell. The use of HEK cells to study neurodegeneration has several obvious limitations. HEK cells are not neurons. That is, they do not develop any of the specialized structures unique to neurons, in particular synaptic connections. HEK cells are an immortalized line, meaning they continue to divide. This is in direct contrast to mature, terminally differentiated neurons. In addition, there are many advantages to the use of HEK cells. The use of human cell culture allows access to a wide range of antibodies and reagents that are often not available for cell cultures from other species such as Drosophila or rat. HEK cells show a high transfection efficiency using plasmid vectors and siRNA knockdown is also similarly efficient. At the same time, these properties facilitate experiments utilizing multiple vector constructs and combinations, this in comparison to the large amount of work and time required to make similar numbers of viral vectors. Large amounts of protein are typically produced, facilitating protein-protein interaction studies. In addition, the cells are easy to culture in large numbers, enabling biochemical and metabolic testing that requires large numbers of transfected cells. Large numbers also allows ease of automated screening, where culture consistency is necessary for accurate results. Thus, HEK293 cells are useful as an 
initial cell to establish the basic biochemistry and metabolism associated with a molecular pathway. Despite their obvious structural differences to neurons, the cells show a similar response to that of neurons, in terms of toxicity to drugs and toxic proteins, making them a good first approximation, before moving to more complicated cell culture systems.

Several other immortalized cell lines in frequent usage are the SH-SY5Y human neuroblastoma and PC12 rat neuroblastoma cells. Both of these cell types can be differentiated to a more neuron-like phenotype after chronic exposure to either growth factors or retinoic acid. The cells express many features found in primary DA neurons, including the DAT, tyrosine hydroxylase vesicles and DA release. After $\alpha$-Synuclein overexpression, cytoplasmic inclusion bodies can be found in both cell types. However, the use of these cells is subject to several limitations. Firstly, plasmid transfection efficiency is very low. If the goal is single cell morphology documentation or microscopy usage, then low transfection efficiency is acceptable. However this limitation makes using the cells for large scale biochemical or apoptotic assays impractical. If such an application is desired, viral vectors are a necessity. At the same time, these cells share a problem common to immortalized cells, that being, it is difficult to distinguish between growth arrest and cell death. In order to determine the conditions necessary to produce cell death versus decreased viability, careful preliminary experiments are necessary for each toxin to be tested. ${ }^{181}$

Primary immature neuron cultures can be harvested from mouse or rat embryos and maintained in culture for several weeks. Use of primary neurons brings the obvious advantages of conducting experiments on a cell most similar to that found in a living animal. In culture, the cells will form neurites and synapses. Of particular importance is that they do not divide and thus experiments measuring cell survival after toxin application or protein expression have the greatest applicability to the in vivo brain. However there are several limitations to using primary neurons. In particular, the cells are not amenable to gene transfer 
using plasmids, again necessitating viral vector usage. Efficacy of the vector in achieving expression depends on the type of neuron infected. Batch to batch variability may be a factor, dependent on the person preparing the cells and the method used for isolation, potentially limiting ease of experimental comparison. In addition, the cells are very sensitive to growth conditions. Small differences in culture conditions can result in large differences in cell health and consistency, again a potential limitation to inter-experimental comparison and consistency. Moreover, total cell numbers isolatable per animal and per preparation are limited, this in comparison to large cell numbers available when using immortalized lines.

Cultures can be prepared from several different brain areas. In particular, embryonic day 13 (E13) mouse or rat embryos can be used to isolate mesencephalic cultures. Lentivirus infection provides a means of transgene expression. There is however a drawback associated with this type of culture. That is, these cultures are typically a highly heterogeneous mixture of glia and neurons, with the actual number of $\mathrm{TH}$ positive cells approximately $5-10 \%$ of the total population. ${ }^{181}$ This limits the usage of viability and biochemical assays, due to the low number of 'target' cells within the entire population. Another culture that can be prepared is a primary cortical culture, this from E18 rat embryos. The usefulness of this culture is that it exhibits a high percentage of neurons to glia (approximately 8:1) when in short term culture with high density seeding (unpublished personal observations). In addition, lentiviral vector infection results high (80-90\%) infection. These aspects make these cells useful for viability assays where high cell numbers expressing the protein of interest and relatively homogenous cultures are required. Thus, use of primary neuron cell culture allows for assessment of viability and microscopic examination, providing a useful proof of principle and mechanistic analysis before moving to mammalian models of PD.

\subsubsection{Drosophila melanogaster model}

As described, Parkinson's is a disease that is causally linked to both genetic and 
environmental factors. However, robust investigation into genetic pathways using epidemiological-based studies is hampered by the complex patterns of inheritance found in humans, typical lack of long term pedigree data and population heterogeneity. Thus, a model system that can be followed from birth to death over multiple generations, that is amenable to genetic alteration and that provides large numbers of genetically similar individuals is needed in order to better investigate the genetics of PD. Drosophila melanogaster is one such system. In particular, there are multiple factors that rationalize the use of the fruit fly to study human disease: they are small, cheap and easy to maintain, enabling wide-screen genetic screening experiments; a short life span of maximum 120 days makes multi- generation experiments feasible; show complex behavior and locomotor ability; have well-characterized anatomy, development and genetics; a multitude of genetic tools and libraries are available; temporal and tissue specific expression of genes of interest can be relatively easily accomplished. ${ }^{182}$ While having a more complex nervous system than the nematode (C. elegens), the fruit fly brain does not approach the perhaps overwhelming complexity of the mammalian brain, thereby enabling structure-function analysis.

Of course, one may question the utility and accuracy of comparing an invertebrate brain to that of Homo sapiens. However, full sequencing of both the fruit fly and human genomes has revealed a relatively high degree of similarity between the two species. ${ }^{183}$ In particular, many of the core function signaling pathways including cell growth and apoptosis, show high levels of evolutionary conservation, with homologous genes regulating homologous pathways. ${ }^{184}$ Thus, multiple models of human neurodegenerative diseases have been generated in the fly including HD, Fragile X Syndrome and Friedrich's ataxia, Amyotrophic Lateral Sclerosis, and AD. ${ }^{182} 185$

PD has also been modeled in Drosophila. Like humans, Drosophila also uses DA as a neurotransmitter involved in motor control. Thus, it is assumed that a reduction in DA would 
also result in locomotor defects, as seen in humans. Several genetic and toxin- based models of PD have been generated in the fly, including expression of mutant or wild type forms of $\alpha-$ Synuclein, PINK1, Parkin, DJ-1 and LRRK2. In particular, loss of function mutations in Drosophila homologues of the following genes: PINKI ${ }^{98,99}$ Parkin $^{186-189}, D J-I^{158,190,191}$, LRRK2 $2^{192,193}$ and HTRA $2^{194-196}$, can all lead to a PD-like phenotype, characterized by DA cell loss and motor defects. Fly studies were the first to demonstrate the importance of PINK1 and Parkin in mitochondrial function and moreover, the interaction of Parkin, PINK1 and DJ-1. An important caveat to these models is that LB-like aggregations are not typically found, with the exception of some reports of mutant $\alpha$-Synuclein overexpression. LB absence may be due the fact that there is no Drosophila homologue to $S N C A$ and thus proteins relating to $\alpha$ Synuclein aggregation may not exist in the fly. That said, LB formation is not always seen in genetic mouse models of PD, where mouse homologues to $\alpha$-Synuclein exist. This suggests LB formation to be a disease feature specific to the primate brain. Both wt and mutated $\alpha$ Synuclein have been expressed in the fly brain or selectively within DA neurons. Expression of wt or mutant $\alpha$-Synuclein again results in a PD-like phenotype, with DA neuronal loss, enhanced sensitivity to oxidative stress and locomotor defects. ${ }^{126,135,142,197,198}$ Expression of $\alpha$-Synuclein[A53T] results in a more severe toxic phenotype. ${ }^{199}$ The toxic phenotype in both man and fly is therefore supportive of the idea that homologous cellular pathways are involved in both human and fly pathogenesis. Fly investigation into the pathogenesis of $\alpha$ Synuclein has yielded data indicating that $\alpha$-Synuclein's S129 phosphorylation state and oligomer production are key to toxicity. In addition, investigation into genetic modifiers of $\alpha$ Synuclein has revealed that the activity of SOD, an important antioxidant enzyme, and calpain, a cytosolic calcium-dependent protease, both influence $\alpha$-Synuclein toxicity. ${ }^{200,201}$ Finally, the potential importance of molecular chaperones in mitigating the toxicity of $\alpha$ - 
Synuclein has been demonstrated, as geldanamycin treatment, which can upregulate HSP70, suppresses $\alpha$-Synuclein toxicity in the fly. ${ }^{197}$

The presence of fly neurodegenerative models allows for both biased and unbiased genetic modifier screening, experiments which are not practical to do in mammals. Biased screening signifies hypothesis-based pre-selection of candidates, which are then tested for causing phenotype enhancement or suppression. A forward genetic or unbiased screen typically involves screening the entire genome for the ability to modify a phenotype of interest. This can be accomplished through use of random mutation or through targeted genetic tools of RNA interference (RNAi), deficiency or known P-element gene disruption lines. In either case, this approach may thus identify new proteins or new pathways that were previously unassociated with the protein of interest. ${ }^{184}$ However, care must be taken when designing genetic screening approaches for neurodegenerative disease in order to exclude for developmental effects. That is, many genetic screens target gene-of-interest expression to the compound eye, where toxicity results in a 'rough eye' phenotype. This phenotype can be easily scored for suppression or enhancement due to genetic modifiers using a light microscope, allowing for high throughput screening. This approach has been used for multiple screens searching for new interaction partners of $\mathrm{AD}$-related proteins tau and $\mathrm{A} \beta .^{202-205}$ However the number of modifiers identified that also had human homologues generated was often low, suggesting that the eye phenotype observed at hatching resulted from genes active during development, unrelated to age-dependent neurodegeneration. ${ }^{182}$ Thus, screening for an age-dependent phenotype might lead to the identification of modifiers applicable to the human disease. In summary, Drosophila models of neurodegeneration in general and PD in particular, provide a useful experimental window into the mechanisms of protein aggregation, oxidative stress and mitochondrial dysfunction. 


\subsection{Rationale and objectives}

While $\alpha$-Synuclein has been definitively linked to PD pathogenesis for a number of years, there remain a multitude of unanswered questions regarding how it results in neurotoxicity and how it interacts with cell signaling pathways and functions. Genetic screening is a useful tool for generation of novel modifiers. However, while screening for modifiers of $\alpha$-Synuclein toxicity has been done in the common screening species of yeast ( $S$. cerevisiae), a simple eukaryotic single cell model and the nematode, an organism with more simplistic nervous system and limited locomotor ability in comparison than the fly, a genetic screen for new modifiers of $\alpha$-Synuclein has not been published for Drosophila. Thus, conducting a genetic screen in Drosophila, an organism with a full DA nervous system, one displaying both locomotor and aging phenotypes, might yield novel modifiers with greater relevance to the human disease.

Project Objective 1: Identify genetic modifiers of $\alpha$-Synuclein[A53T] toxicity using a genome-wide screen in $D$. melanogaster.

In order to better select for modifiers involved in aging-dependent neurodegeneration, a PD-specific phenotype of toxicity will be used. That is, a decrease of brain DA, which in humans results in the key PD motor dysfunction symptoms and is indicative of withdrawal of SNc projections to the striatum, precedes loss of DA neurons. Thus, aging-dependent loss of DA from flies expressing $\alpha$-Synuclein[A53T] in the fly brain will be compared to flies expressing both $\alpha$-Synuclein and carrying a chromosomal deletion, resulting in decrease of certain gene products. Fly DA measurements will be made using high performance liquid chromatography (HPLC). Use of $\alpha$-Synuclein[A53T] is rationalized on the basis that this mutant has consistently resulted in the most severe PD phenotype in comparison to both wt and other familial point mutations. Use of the most severe mutant allows for creation of a more robust phenotype for screening. Flies from the Bloomington Deficiency Kit, each 
carrying a specific chromosomal deletion, will be used for initial crossing. An enhanced loss of DA resulting from a specific deficiency line cross is therefore suggestive that loss of a gene product contained within the deficiency enhances toxicity. In order to find the specific gene resulting in the modification of $\alpha$-Synuclein toxicity, single gene RNAi and P-element gene disruption lines available from public Drosophila stock collections will be used.

Project Objective 2: Clarify the robustness, specificity and mechanism of action of novel modifiers identified.

Secondary screening endpoints (locomotor ability assessment, oxidative stress sensitivity, longevity and loss of DA neurons) associated with PD phenotype evaluation will be used to clarify the robustness of any found interaction. In addition, immortalized cell lines and primary neuron cultures may provide an additional opportunity to investigate more specific aspects of novel interactions. 


\section{Methods and Materials}

All laboratory chemicals were obtained from Sigma Aldrich, Germany, unless other noted. All recombinant DNA techniques were performed according to protocols described in the Molecular Cloning laboratory manual unless otherwise noted. ${ }^{206}$ Please see Methods section 3.3 for a summary of all basic laboratory buffers used.

\subsection{Fly experiments}

\subsubsection{Fly Stocks and transgenic fly expressing human TRAP1 generation}

All flies were maintained on normal cornmeal-yeast-molasses-agar based fly food in a $25^{\circ} \mathrm{C}$ room unless otherwise noted. For explanation of the GAL4-UAS driver system used for directed expression of transgenes and RNAi; please see Fig.III-1. All non-RNAi stocks were obtained from the Bloomington Drosophila Stock Centre (University of Indiana, Bloomington, IN, USA). All UAS-RNAi stocks were obtained either from the Vienna Drosophila RNAi Center (VDRC, Institute for Molecular Pathology, Vienna, Austria) or from NIG-FLY (Fly Stocks of the National Institute of Genetics, Mishima, Japan). Basic stocks from Bloomington used were: $\mathrm{w}^{1118} ; ; \mathrm{P}\{$ Ddc-GAL4.L $\} 4.36 \quad$ (BL 7009: ddc=dopa decarboxylase, aminergic neuron specific driver, referred to in text as ddc-GAL); w ; P $\{$ UASlacZ.B \}Bg4-2-4b (BL 1777; referred to in text as UAS-LacZ); ${ }^{*}$; P\{croc-lacZ.7\}3, croc ${ }^{5 F 59}$ $\mathrm{e}^{*} / \mathrm{TM} 3, \mathrm{Sb}^{1}$ (BL 7103: referred to in text as w; croc/TM3, Sb). Stock containing: elavGAL4 driver (pan-neuronal) ( $\mathrm{w}^{1118} ;$; elav-GAL4, referred to as elav-GAL flies in text) on the third chromosome and white flies $\left(\mathrm{w}^{1118}\right)$ were gifts from the laboratory of Prof. Herbert Jäckle, Max Planck Institute for Biophysical Chemistry, Göttingen, Germany. A fly line expressing GFP under the ddc driver (w; ddc-GAL4>UAS-GFP) was generated by Dr. Aaron Voigt (Dept. of Neurology, Aachen University Clinic, Aachen, Germany). UAS-GFP chromosome were created via germ line transmission using the EMBL service and then recombined with ddc-GAL4 driver (flies referred to as ddc>GFP in text). Wild type flies 
(Oregon R; referred to as + in text) were a gift from the laboratory of Prof. Herbert Jäckle,

Max Planck Institute for Biophysical Chemistry, Göttingen, Germany.

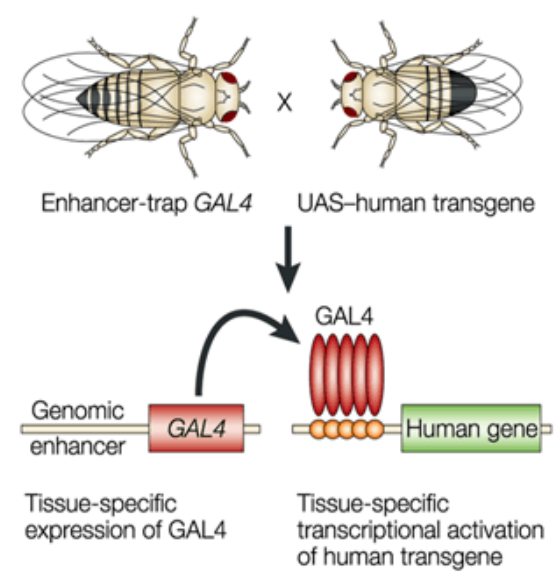

Fig. III-1. Drosophila UAS-GAL4 transgene expression system. In order to achieve tissue- specific transgene expression, a two part (UAS, upstream activating sequence and GAL4, yeast transcription activator protein) can be used. The GAL4 gene is inserted into the Drosophila genome and placed under the control of a native gene promoter. The UAS binding sequence is located upstream of an inserted transgene. Without binding of GAL4 to the UAS, the human transgene will not be expressed. GAL4 will only be expressed under tissue-specific activation. Various tissue specific promoters have been combined with GAL4 to create 'driver' lines: in particular, ddc-GAL4, with specific expression in dopaminergic neurons and elav-GAL4, with pan-neuronal expression.

Figure from Muqit et al. Modeling neurodegenerative diseases in Drosophila: a fruitful approach? Nature Reviews Neuroscience. 2002;3: 237-243.

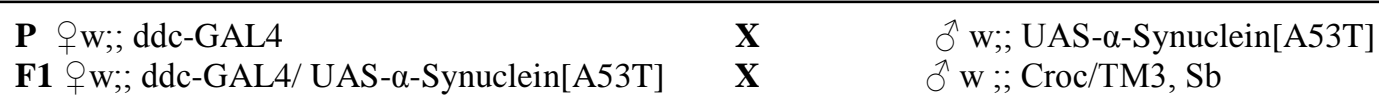

Possible chromosomal recombination event. Select male offspring with the stubble marker for F2 breeding and breed single male fly to female balancer flies.
F2 + w; $; \operatorname{croc} / \mathrm{TM} 3, \mathrm{Sb}$
$\mathbf{X}$
ô $\mathrm{w} ;$ ? ?/TM3, Sb

After F2 breeding, single fly PCR (see 3.1.2. for protocol) on male candidates was performed, to check for presence of both GAL4 and $\alpha$-Synuclein[A53T]. Confirmation of GAL4 and $\alpha$-Synuclein in one fly enabled utilization of offspring from F2 to create balanced breeding stock.

F3 + w; $;$ ddc-GAL4> UAS- $\alpha$-Synuclein[A53T]/TM3, Sb $\quad \mathbf{X} \quad \delta$ w; ddc-GAL4> UAS- $\alpha-$ Synuclein[A53T]/ TM3, Sb

In order to generate a breeding stock containing both driver and human $\alpha$-Synuclein [A53T] (yw;; UAS- $\alpha-$ Synuclein[A53T] flies created by Madhu Babu, Max Planck Institute for Biophysical Chemistry, Göttingen, Germany) (flies referred to in figures as UAS-A53T) ${ }^{141}$ on a single chromosome for ease of use, chromosomal recombination was exploited using the 
above outlined breeding schematic. Flies created hereafter referred to in figures as ddc>A53T flies. The process shown below was repeated using the elav-GAL driver (referred to in figures as elav $>$ A53T flies).

TRAP1 specific flies used were obtained from both Bloomington and NIG-FLY. In particular, a P-element TRAP1 gene disruption line BL14032 was obtained from Bloomington ( $\mathrm{y}^{1} \mathrm{w}^{67 \mathrm{c} 23}$; $\mathrm{P}\{\mathrm{SUPor}-\mathrm{P}\}$ Trap1 ${ }^{\mathrm{KG} 06242}$; referred to in text as TRAP1[KG06242]). Third chromosome insertion RNAi fly stocks against TRAP1 from NIG FLY were: 3152R1 and 3152R3 (flies referred to in text as TRAP1-RNAi[3152R1] and TRAP1-RNAi[3153R1]).

To generate a transgenic fly expressing human TRAP1, human TRAP1 cDNA (see cell culture methods section 3.2.1. for isolation of human TRAP1 cDNA) was sub-cloned from pcDNA3.1+ vector into pUASattB (vector for site directed integration using $\phi_{\mathrm{C}} 31$ integrase $)^{207}$ using the KpnI and XbaI restriction sites. Human TRAP1 in pUASattB was sequenced and the pUASattB-TRAP1 vector was then sent to BestGene Inc (Chino Hills, CA, USA) for fly embryo injection and cytosite insertion (insertion cytosite 76A2). Flies received from BestGene were checked for human TRAP1 expression using Western blotting (see 3.1.10 for protocol and antibody) and balanced using w; croc/TM3,Sb flies before using for crossing (w;; UAS-human TRAP1/TM3, Sb). Fly referred to in text as hTRAP1.

\subsubsection{Single fly polymerase chain reaction (PCR) for genotyping}

A single fly was placed in $50 \mu$ of cold extraction buffer $(10 \mathrm{mM}$ Tris- $\mathrm{Cl} \mathrm{pH} 8.2,1$ $\mathrm{mM}$ EDTA, $25 \mathrm{mM} \mathrm{NaCl})$ containing proteinase $\mathrm{K}(2 \mathrm{mg} / \mathrm{ml}$; P-2308, Sigma-Aldrich, Germany). A pipette tip was used to homogenize the fly and was followed by 30 minutes of incubation at room temperature (RT). Proteinase $\mathrm{K}$ was then inactivated by incubation at $92^{\circ} \mathrm{C}$ for 2 minutes.

$50 \mu 1$ PCR reactions were prepared using the following proportions:

\begin{tabular}{|c|c|}
\hline Volume $(\mu \mathrm{l})$ & Component \\
\hline 1 & Fly extract \\
\hline 5 & 10X PCR Buffer $\left(160 \mathrm{mM}\left(\mathrm{NH}_{4}\right)_{2} \mathrm{SO}_{4}, 670 \mathrm{mM}\right.$ Tris- \\
\hline
\end{tabular}




\begin{tabular}{|c|c|}
\hline & $\begin{array}{l}\mathrm{HCl} \mathrm{pH} \mathrm{8.8,15} \mathrm{mM} \mathrm{MgCl} 2,0.1 \% \text { Tween-20) } \\
\text { (Genecraft, Germany) }\end{array}$ \\
\hline 1 & 10 mM dNTP (Fermentas Life Sciences, Germany) \\
\hline $1-1$ & Primers at $10 \mathrm{pmol} / \mu \mathrm{l}$ \\
\hline 0.25 & $\begin{array}{l}\text { Taq polymerase (Biotherm DNA Polymerase, } \\
\text { Genecraft) }\end{array}$ \\
\hline 40.75 & $\mathrm{ddH}_{2} \mathrm{O}$ \\
\hline
\end{tabular}

Primer sequences:

GAL4-for: 5 - atgaagctactgtcttctatcg-3`

rev: 5 - aagagcatccetgggcataaa -3

a-Synuclein for: 5 - ggggtaccatggatgtattcatgaaag-3`

rev: 5 - ttaggcttcaggttcgtagtc-3`

Conditions for PCR carried out using a Mastercycler Gradient (Eppendorf, Germany) thermocycler were as follows:

\begin{tabular}{|l|l|l|l|}
\hline Step & Temperature $\left({ }^{\circ} \mathbf{C}\right)$ & $\begin{array}{l}\text { Duration (minutes or } \\
\text { seconds (s)) }\end{array}$ & Repeat \\
\hline Initial Denaturation & 94 & 5 & 1 \\
\hline Denaturation & 94 & $30 \mathrm{~s}$ & 30 \\
Primer Annealing & 55 & $30 \mathrm{~s}$ & \\
Elongation & 72 & 1 & 1 \\
\hline Final Elongation & 72 & 10 & 1 \\
\hline
\end{tabular}

PCR products were then separated using agarose gel DNA electrophoresis. Samples were combined with 6X Loading Buffer and loaded onto 1\% agarose gels containing $0.0001 \%$ ethidium bromide (Sigma-Aldrich). A DNA ladder of appropriate size (Generuler $1 \mathrm{kB}$ DNALadder, Fermentas) was also loaded on gels as point of comparison. Gels were visualized using the Biovision video documentation system (Peqlab Biotechnology, Germany).

\subsubsection{Treatment of flies with $\alpha$-methyl tyrosine}

Wild type male flies were collected at time of hatching and placed in empty vials containing filter paper soaked in either 5\% sucrose solution alone or 5\% sucrose solution with $5 \mathrm{mM} \alpha$-methyl tyrosine (MT). The filter paper was treated each day with the necessary solution. Flies were collected for HPLC measurements at day 0, 2, 4, 6 and 8 (20 flies per sample). HPLC was then used to measure fly head DA (see 3.1.5.).

\subsubsection{Deficiency screen breeding}


The Bloomington Deficiency Kit (Indiana, US, http://flystocks.bio.indiana.edu/) was utilized for screening purposes. Each line lacks a defined chromosomal section. Deficiencies were selected such that almost the entire genome was covered. Bloomington lines used can be seen in Appendix Results, Table 1. In general, male flies from a specific deficiency line were crossed to virgin females:

q w; ddc-GAL> UAS- $\alpha-$ Synuclein[A53T]/ TM3, Sb

Male offspring containing the requisite absence/presence of selectable markers (depending on deficiency line) were isolated and aged. At ages 1 and 4 weeks, a minimum of 9 flies were collected for later measurement of brain DA using HPLC. For deficiencies on the $\mathrm{X}$ chromosome, virgins were collected and bred to male flies containing ddc>A53T and offspring containing the requisite deficiency plus ddc>A53T copy were collected and aged, as above.

\subsubsection{Measurement of fly brain DA using HPLC}

Fresh fly samples were flash frozen in liquid nitrogen and stored at $-80^{\circ} \mathrm{C}$. The samples were first placed in liquid nitrogen and all future processing was done on ice or at $4{ }^{\circ} \mathrm{C}$. Frozen fly samples were vortexed, allowing head removal from fly body using tweezers. Fly heads were placed in homogenization buffer (3 fly heads, $100 \mu \mathrm{l}$ of $0.1 \mathrm{M}$ perchloric acid/ 3\% trichloric acid solution) in a $2 \mathrm{ml}$ tube containing ceramic beads $\left(\mathrm{ZrO}_{2}, 1.2-1.4 \mathrm{~mm}\right.$ beads from Mülmeier Mahltechnik, Germany) and homogenized using a Percellys 24 Lysis and Homogenization machine (6500 revolutions per minute (rpm) for 30 s; Peqlab, Germany), followed by a brief centrifugation to enable transfer of samples to fresh tubes. Samples were then spun at $14,000 \mathrm{~g}$ for 15 minutes at $4^{\circ} \mathrm{C}$. Fifty microlitres of supernatant from each sample was used for HPLC analysis (Dionex Ultimate 3000 machine; running buffer of $57 \mathrm{mM}$ citric acid, $43 \mathrm{mM}$ sodium acetate, $0.1 \mathrm{mM}$ EDTA, $1 \mathrm{mM}$ octane sulfonic acid and $20 \%$ methanol) (Dionex, Germany). The samples were first separated on a chromatographic column (Dionex 
Acclaim C18, 5um, 2.1x $150 \mathrm{~mm}$ column, at $25^{\circ} \mathrm{C}$ ) and then electrochemically detected on a graphite electrode (Dionex ED50 Electrochemical detector with the following conditions: disposable carbon electrode at $0.8 \mathrm{~V}$, flow rate $0.2 \mathrm{ml} / \mathrm{min}$ ). DA (Sigma-Aldrich, Germany) standards of $0.1 \mu \mathrm{M}, 0.25 \mu \mathrm{M}$ and $0.4 \mu \mathrm{M}$ were included in each HPLC run for creation of a standard curve. Chromeleon 6.6 software was used for HPLC data analysis. DA values for each deficiency line are indicated (4 week average value/ 1 week average value).

\subsubsection{Fly longevity and oxidative stress assays}

For longevity assays, male flies were collected within 2-3 days of hatching and maintained at $25^{\circ} \mathrm{C}$ in vials with normal fly food (not more than 20 flies per vial). Flies were transferred to new food twice per week and scored for survival until all flies had died. A minimum of 20 flies were counted for all longevity assays. For initial fly longevity experiments comparing flies with either 1 or 2 copies of $\alpha$-Synuclein[A53T], single copy flies were: $\mathrm{ddc}>\mathrm{A} 53 \mathrm{~T} /+$ and double copy flies were $\mathrm{ddc}>\mathrm{A} 53 \mathrm{~T} / \mathrm{ddc}>\mathrm{A} 53 \mathrm{~T}$ (the same for the elavGAL4 driver).

For the hydrogen peroxide $\left(\mathrm{H}_{2} \mathrm{O}_{2}\right)$ assay, male flies were collected within 2 days of hatching and placed within a vial containing filter paper treated with $2.4 \% \mathrm{H}_{2} \mathrm{O}_{2}$ in a $5 \%$ sucrose solution. The filter paper was treated with $\mathrm{H}_{2} \mathrm{O}_{2}$-sucrose solution daily and survival scored until all flies died.

For the paraquat assay, paraquat dichloride $(20 \mathrm{mM}$ in a $5 \%$ sucrose solution) was applied to filter paper. Newly hatched male flies were placed in paraquat-filter vials at $25^{\circ} \mathrm{C}$ and survival scored at 6-12 hour intervals until 60 hours following treatment. A minimum of 20 flies were counted per genotype for all assays. ${ }^{208}$

\subsubsection{Negative geotaxis assay (fly climbing)}

Fly climbing was assessed similar to previously published protocols. ${ }^{136,142,135,209}$ A group of flies was aged on normal yeast medium and at ages 1 and 4 weeks, climbing was 
assessed (twenty flies per genotype). Flies were individually placed in a graduated cylinder, and allowed to climb for $15 \mathrm{~s}$. Maximum height attained was recorded and analysis was repeated 3 times per time point, with 3 trials at one minute intervals recorded at each time point.

\subsubsection{Whole fly brain stain for tyrosine hydroxylase and neuron counting}

Fly brains were carefully dissected in cold PBS under a Leica SMZ-168 dissecting microscope and then washed in a PBS/ 0.1\% Triton X (PBT) solution under rotation. Brains were then fixed in $4 \%$ paraformaldehyde (PFA) $\left(30\right.$ minutes, $\left.4^{\circ} \mathrm{C}\right)$, washed in $\mathrm{PBT}(4 \mathrm{C}, 3 \mathrm{x} 15$ minutes) and then blocked in PBT containing 5\% normal goat serum (overnight, $4^{\circ} \mathrm{C}$ ). Flies expressing ddc >GFP were first assessed for GFP expression, and specificity of ddc-GAL4 driver was checked using staining for tyrosine hydroxylase (TH). Later experiments used only anti-TH staining for neuron counting. For TH staining, brains were incubated with a primary anti-TH antibody (1:100 in PBT+ 5\% goat serum; rabbit polyclonal, AB152, Chemicon International/ Millipore, Germany) for 2 days, $4{ }^{\circ} \mathrm{C}$. Following washing in PBT $(3 \mathrm{x} 15$ minutes), brains were incubated with the fluorescent secondary anti-rabbit antibody (1:200 in $\mathrm{PBT}$ at RT for 3.5 hours; AlexaFluor-555 or Cy3; Invitrogen, Germany/Jackson Immunological Research, England). After washing in PBT, brains were mounted in Vectashield (Vector Labs, UK). The number of TH positive neurons was determined via $1 \mu \mathrm{m}$ optical sectioning and creation of $\mathrm{Z}$ stack images on a confocal microscope (Leica DM IRE2, Laser). ${ }^{210} \mathrm{Cy} 3$ or AlexaFluor-555 fluorescence was imaged using a $488 \mathrm{~nm} \mathrm{Ar} / \mathrm{Kr}$ laser and 500-550 nm band pass emission filter. Each fluorescent image was acquired individually (serial sequential scanning, $1 \mu \mathrm{m}$ height, 1024x1024 resolution, $200 \mathrm{~Hz}$ ), and then averaged and merged with the Leica Confocal Software. At least 15 brains were analyzed per genotype.

\subsubsection{Protein collection from fly heads}


Flies were placed in tubes and flash frozen in liquid nitrogen. Frozen flies were briefly vortexed to separate fly heads from bodies. Heads were placed in vials containing RIPA buffer $(25$ heads in $100 \mu \mathrm{l})$ and a small quantity of ceramic beads on ice. The samples were then homogenized using the Precellys 24 machine (20 s, 5000 rpm). Following homogenization, a brief spin was used to pellet the beads. The supernatant was collected and cleared by an additional centrifugation for $10 \mathrm{~min}\left(12000 \mathrm{rpm}, 4^{\circ} \mathrm{C}\right)$. Protein concentration was measured using the BioRad Protein Assay Reagent (BioRad Laboratories, Germany).

\subsubsection{Protein Western blotting}

For Western blot analysis, protein samples were diluted in loading buffer ( $2 \mathrm{X}$ Laemmli buffer) and boiled $\left(95^{\circ} \mathrm{C}, 5\right.$ minutes), before loading and separation via SDS-PAGE gel (polyacrylamide gel of either 10, 12 or $15 \%$ run for $80 \mathrm{~V}, 2$ hours) and subsequent transfer onto nitrocellulose membrane ( $80 \mathrm{~V}, 1$ hour). The membranes were then blocked with skim milk (1 hour, 5\% skim milk in TBST: $0.05 \mathrm{M}$ TBS containing 0.1\% Tween-20). After blocking, membranes were incubated overnight at $4^{\circ} \mathrm{C}$ with primary antibodies.

The primary antibodies used were as follows:

\begin{tabular}{|l|l|l|}
\hline Antibody & Dilution & Company Source \\
\hline mouse anti- $\alpha$-Synuclein & $1: 1000$ & Cell Signaling, Germany \\
\hline mouse anti- Drosophila CSP3 & $1: 700$ & $\begin{array}{l}\text { Developmental Studies Hybridoma Bank, University of } \\
\text { Iowa, Iowa, USA }\end{array}$ \\
\hline mouse anti- $\delta$ tubulin & $1: 10,000$ & Sigma Aldrich \\
\hline $\begin{array}{l}\text { rabbit anti- cleaved Caspase - } \\
\text { polyclonal }\end{array}$ & $1: 1000$ & Cell Signaling \\
\hline mouse anti- TRAP1 & $1: 1000$ & BD Biosystems, Germany \\
\hline $\begin{array}{l}\text { rabbit anti- p44/42 MAPK } \\
\text { polyclonal }\end{array}$ & $1: 1000$ & Cell Signaling \\
\hline $\begin{array}{l}\text { mouse anti- phospho-tyrosine } \\
\text { (PY99) }\end{array}$ & $1: 200$ & Santa Cruz Biotechnology, Germany \\
\hline $\begin{array}{l}\text { mouse anti- phospho-threonine } \\
\text { (H2) }\end{array}$ & $1: 200$ & Santa Cruz Biotechnology \\
\hline mouse anti- phospho-serine (H2) & $1: 200$ & Santa Cruz Biotechnology \\
\hline mouse anti- Cytochrome c & $1: 500$ & Santa Cruz Biotechnology \\
\hline rabbit anti- VDAC1 & $0.3 \mu \mathrm{g} / \mathrm{ml}$ & Abcam, UK \\
\hline mouse anti- COX IV & $2 \mu \mathrm{g} / \mathrm{ml}$ & Abcam \\
\hline
\end{tabular}


\begin{tabular}{|l|l|l}
\hline rabbit anti- GFP (FL) polyclonal & 1:1000 & Santa Cruz Biotechnology \\
\hline
\end{tabular}

Appropriate secondary anti- mouse or rabbit horse radish peroxidase-linked antibodies $(1: 10,000)$ were obtained from GE Healthcare (Germany) and incubated for 1 hour after washing three times in PBT. Blots were once again washed three times in PBT before signal detection using the Chemiglo substrate (Biozym, Germany), with imaging on the Alpha Imager (Alpha Innotech/ Biozym, Germany).

\subsubsection{Total RNA isolation and cDNA preparation from fly heads}

Method was adapted from Bertucci et al. ${ }^{211}$ Twenty flies were collected in a $1.5 \mathrm{ml}$ tube and flash frozen in liquid nitrogen. The samples were removed briefly vortexed to separate fly heads from bodies. Heads were placed in a tube containing Total RNA Isolation Reagent (Abgene, Germany) and homogenized using a sterilized plastic pestle for several minutes, then incubated at RT for 5 minutes. A small volume of chloroform was added to the homogenate, followed by $30 \mathrm{~s}$ of vigorous shaking. After a 10 minute incubation at RT, samples were centrifuged (15 minutes, $14,000 \mathrm{rpm}$ ) and the aqueous layer was transferred to a new $0.5 \mathrm{ml}$ tube. RNA was precipitated using isopropanol and Glycoblue (Ambion, Germany). After centrifugation (15 minutes, 14,000 rpm), the supernatant was discarded, the pellet was washed in $75 \%$ cold ethanol, followed by a brief centrifugation and ethanol removal. The RNA pellet was re-suspended in RNA-free water and the concentration determined using a NanoDrop1000 spectrophotometer (Thermo Fisher Scientific, UK). RNA samples were treated with DNase following manufacturer's instructions (Promega RQ1 RNase-Free DNase kit; Promega Germany). cDNA production via reverse transcription was performed using the iScript cDNA Synthesis Kit according to the manufacturer provided instructions (BioRad). cDNA concentrations were determined using the NanoDrop1000 spectrophotometer.

\subsubsection{Real-time PCR with fly samples}


Real-time PCR measurements were performed using the SYBR Green (Thermo Fisher Scientific) reagent following manufacturer's instructions for preparation of PCR samples and the MX3000p qPCR machine (Stratagene/Agilent, Germany). Gene of interest signal was compared to that of control gene expression ( $\beta$-actin5c) using the $2^{-\Delta \Delta \mathrm{Ct}}$ method. ${ }^{212}$ No-RT controls were performed to exclude for genomic DNA sample contamination. PCR reactions were followed by generation of a dissociation curve to check for side product generation. Amplification conditions were as follows:

\begin{tabular}{|l|l|l|l|}
\hline Step & Temperature $\left({ }^{\circ} \mathbf{C}\right)$ & Duration (minutes or s) & Repeat \\
\hline Initial Denaturation & 95 & 5 & 1 \\
\hline Denaturation & 95 & $30 \mathrm{~s}$ & 40 \\
Primer Annealing & 58 & $30 \mathrm{~s}$ & \\
Elongation & 72 & 1 & \\
\hline Final Elongation & 72 & 10 & 1 \\
\hline
\end{tabular}

The primers used were as follows:

Human $\alpha$-Synuclein:

for: 5 '- agaagacagtggagggagca -3 '

rev: $5^{\prime}$ - tccagaattccttcctgtgg -3 '

Fly $\beta$-actin5c:

for: $5^{\prime}$-ccagtcattcctttcaaacc-3'

rev: 5'-gcaacttcttcgtcacacatt-3'

Fly TRAP1:

for: 5'-aggcagagtcaccgatcc-3'

rev: $5^{\prime}$-tgatgectgcttggtctc- $3^{\prime}$

Fly citrate synthase $(\mathrm{kdn})$ :

for: 5'-gtcgctctatcgcatttcc-3'

rev: 5'-cgaacataggctcccacat-3'

\subsubsection{Measurement of citrate synthase (CS) enzymatic activity from fly lysates}

The protocol for measuring CS activity was adapted from a protocol provided by SigmaAldrich with their CS assay kit (CS0270). In $50 \mu$ cold extraction buffer $(0.1 \%$ Triton X-100, $1 \mathrm{mM}$ EDTA, $20 \mathrm{mM}$ HEPES, pH 7.2), three fly heads were homogenized, flash frozen in liquid nitrogen and centrifuged at high speed to pellet cellular debris. Protein-containing 
supernatant was then removed to a fresh tube and assayed for protein content using the Bradford assay. Samples in triplicate were prepared at RT in 96-well plate for assay on a kinetic plate reader (Tecan Rainbow, SpectraFluor Plus, Tecan Deutschland GmbH) set to measure at $412 \mathrm{~nm}$. Each sample contained of total $100 \mu \mathrm{l}$ with $10 \mu \mathrm{l}$ of sample with acetyl CoA $(3 \mathrm{mM})$ and DTNB (1 $\mathrm{mM})$. Data was collected for 1.5 minutes to establish baseline, before addition of oxaloacetic acid (OAA) $(10 \mathrm{mM})$ to the plate. Data was then collected for 10 minutes at 15 s intervals. Signal was confirmed using a positive control of CS (enzyme from Sigma Aldrich). Absorbance vs. time was plotted, and absorbance per minute was calculated for endogenous (before OAA addition) and total activity (after OAA). Net CS activity was thus total activity subtracted from endogenous, and divided by protein concentration (final CS activity in $\mu \mathrm{mole} / \mathrm{ml} / \mathrm{min} / \mathrm{mg}$ protein).

\subsection{Cell culture}

\subsubsection{Cloning and in vitro mutagenesis}

Full length human $\alpha$-Synuclein cDNA (423bp), with the [A53T] mutation (cDNA a gift from Dr. Felipe Opazo, European Neuroscience Institute, Göttingen, Germany) was subcloned into the pcDNA3.1+ expression vector (Invitrogen) using the HindIII and EcoRV (Fermentas restriction enzymes) restriction sites located within the multiple cloning site, downstream of the CMV promoter. Restriction digest cut cDNA and plasmids were isolated using the QiaQuick Gel and PCR Extraction Kits (Qiagen). Cut cDNA and plasmid were then ligated overnight at $16^{\circ} \mathrm{C}$ using T4 DNA ligase (Fermentas). Plasmid amplification was carried out in DH-5 $\alpha$ E. coli bacteria (Invitrogen). Heat shock transformation of DH-5 $\alpha$ was performed according to manufacturer's instructions. Briefly: $50 \mu 1$ of DH-5a mixed with $5 \mu 1$ of ligation reaction followed by 30 minute incubation on ice; heat shock at $42^{\circ} \mathrm{C}$ for $45 \mathrm{~s}$ followed by 2 minutes on ice; bacteria mixed with $250 \mu \mathrm{l}$ SOC medium (Invitrogen) and incubated for 30 minutes at $37^{\circ} \mathrm{C}$; cells plated on LB-Ampicillin plates and incubated 
overnight at $37^{\circ} \mathrm{C}$. Bacteria from single colonies were then amplified in $1 \mathrm{ml}$ of LBAmpicillin liquid for 4 hours at $37^{\circ} \mathrm{C}$ and resulting colonies were analyzed for presence of the plasmid+ insert using PCR (5 $\mu$ l of bacteria in a PCR reaction using T7 and BGH Reverse primers, primers N560-02 and N575-02 from Invitrogen). Positive colonies were then expanded overnight into $100 \mathrm{ml} \mathrm{LB}$-Ampicillin liquid cultures and plasmids were isolated using the Qiagen Mini Prep kit/ Qiafilter Plasmid (Qiagen).

Full length human TRAP1 cDNA (2115bp) was amplified from human HEK 293 cell cDNA samples and restriction enzyme sites of BglII and XhoI introduced to the sequence using the following primers:

For: 5'-gaagatctatggcgcgcgagctgcgggcgctgctgc-3', rev: 5'- ccgctcgagtcagtgtcgctccagggccttgaca-3'.

Correct cDNA sequence was confirmed with sequencing (StarSeq GmbH, Germany). Sequencher software (version 4.1 from Gene Codes Corporation, Ann Arbor, USA) was used to compare experimental sequencing data to published sequence data (from Pubmed). TRAP1 cDNA was then cloned into the pcDNA3.1+ vector using the BglII, XhoI sites, downstream of the CMV promoter (TRAP1 cDNA isolation from HEK293 done by Dr. Ellen Gerhardt (Dept. Neurodegeneration and Restoration, University of Göttingen, Göttingen, Germany). The plasmid was then amplified using DH-5 $\alpha$ cells as described above.

In vitro mutagenesis of human TRAP1 in pcDNA3.1+ was carried out using the QuickChange Site-Directed Mutagenesis kit (Stratagene) following manufacturer's instructions. Sites for mutagenesis were based on conserved sites found in the ATPase domain (see Appendix Methods Figures A1-1, 2 for sequence homology) and on known phosphorylation sites (see Fig. A1-3). PCR cycling parameters were used as suggested by Stratagene, with a specific extension time of 8 minutes and 16 cycles for all reactions. The kit provided cells (XL1-Blue Supercompetent) were used according to included protocol. Primers used for generating the mutants were as follows: 
TRAP1[D158N] for: 5' - ggcaccatcaccatccagaatactggtatcggg-3

TRAP1[D158N] rev: 5' - cccgataccagtattctggatggtgatggtgcc-3'

TRAP1[T494A] for: 5'-catgcgggecggcgeccgcaacatctactac $-3^{\prime}$

TRAP1[T494A] rev: 5'-gtagtagatgttgcgggcgccggccegcatg -3'

TRAP1[Y498G] for: 5'-caccegcaacatcggctacctgtgcgcc -3'

TRAP1[Y498G] rev: 5'-ggcgcacaggtagccgatgttgcgggtg -3'

Sequence of the mutants was confirmed using automated sequencing (StarSeq $\mathrm{GmbH}$ ) with the listed sequencing primers:

TRAP1 rev1: 5'- tcgctggaaaactccttg c-3'

TRAP1 for2: $5^{\prime}$-gaggacattccctgaacct-3

New plasmids were then amplified using DH-5 $\alpha$ cells as described above.

\subsubsection{Lentivirus preparation}

Full length human TRAP1 cDNA was subcloned into a third generation lentiviral vector pRRLsin.cPPT.PGK/GFP.WPRE (Tronolab, Lausanne, Switzerland), excluding the GFP cassette (virus: pRRLsin.cPPT.PGK-TRAP1.WPRE with phosphoglycerate kinase promoter). The GFP expressing virus served as a control. The correct sequence of TRAP1 within the virus was verified with automated sequencing (StarSeq $\mathrm{GmbH})$. Viruses containing full length human $\alpha$-Synuclein[A53T] and GFP had been previously generated and sequenced using the same method. All viruses were prepared by Dr. Ellen Gerhardt (University of Göttingen, Dept. Neurodegeneration).

\subsubsection{Cells, plasmid transfection and viral infection}

Human HEK293 cells were grown in Dulbecco's modified Eagle's medium (DMEM) and supplemented with $10 \%$ fetal calf serum (Gibco/Invitrogen, catalogue: 10270-106), 100 units $/ \mathrm{ml}$ penicillin, and $100 \mathrm{mg} / \mathrm{ml}$ streptomycin, in a humidified atmosphere of $5 \% \mathrm{CO} 2 /$ $95 \%$ air at $37^{\circ} \mathrm{C}$. 
Transfection of plasmids and small interfering RNA (siRNA) into HEK293 cells was completed using Metafectene (Biontex), following manufacturer's instructions. siRNAs used for gene knockdown experiments were obtained from Qiagen: MAPK1 control siRNA (Qiagen 1027277); 2 different TRAP1 siRNAs (Flexitube siRNA SI03066364 and siRNA SI00115150) and a scrambled siRNA for control (Allstars Negative Control, 1027280). MAPK1 control siRNA was originally used to test concentrations of siRNA to be transfected (see Appendix Methods Fig.A1-S2). Final concentration of siRNA used was $10 \mathrm{nM}$. Cells were seeded on poly-L lysine (PLL) coated plates $\left(35,000\right.$ cells $\left./ \mathrm{cm}^{2}\right)$ and then transfected 48 hours before experimentation.

Primary cortical rat neurons were prepared from E18 rat embryos, following previously published procedures ${ }^{213}$ (neurons prepared by Ulrike Schöl, laboratory of Dr. Sebastian Kugler, Dept. of Neurology, University of Göttingen, Göttingen, Germany). Neurons were seeded on poly-ornithin-coated 24-well plates at a density of 125,000 cells/ $\mathrm{cm}^{2}$. Cells were maintained in Neurobasal medium (Gibco/Invitrogen), supplemented with transferrin (5 $\mu \mathrm{g} / \mathrm{ml}$; Sigma), PSN (1\%, Gibco/Invitrogen), L-Glutamine (0.5 mM; Seromed, Vienna, Austria), B27 supplement (2\%; Gibco/Invitrogen) and media changed every 3-4 days. Primary neurons were infected equimolarly with lentiviruses one day after isolation and then cultured for 6 days before experimental use.

\subsubsection{Total RNA preparation from cell culture and real-time PCR}

Total RNA was prepared from treated and untreated cells using Qiagen RNeasy Mini kit (Qiagen) according to the manufacturer's instructions. cDNA synthesis was carried out as described above for fly RNA (section 3.1.11.). Real time PCR was performed as listed above for fly cDNA (section 3.1.12), however gene of interest was normalized to control 18S signal. Amplification conditions were as follows:

\begin{tabular}{|l|l|l|l|}
\hline Step & Temperature $\left({ }^{\circ} \mathbf{C}\right)$ & Duration (minutes or s) & Repeat \\
\hline Initial Denaturation & 95 & 5 & 1 \\
\hline Denaturation & 94 & $30 \mathrm{~s}$ & 40 \\
\hline
\end{tabular}




\begin{tabular}{|l|l|l|l|}
\hline Primer Annealing & 60 & $30 \mathrm{~s}$ & \\
Elongation & 72 & 1 & 1 \\
\hline Final Elongation & 72 & 10 & 1 \\
\hline
\end{tabular}

Primers were as follows:

Human $\alpha$-Synuclein:

for: 5'- agaagacagtggagggagca -3 '

rev: 5' - tccagaattccttcctgtgg -3 '

Human TRAP1:

for: 5'-cagaccaatgccgagaaag-3'

rev: 5' -caccagctcttcctgtgtca-3'

Human MAPK1:

for: 5 '-taatacgactcactataggg-3'

rev: 5'-tagaaggcacagtcgagg-3'

Human 18S primers: Quantitect primers (Qiagen, Germany).

\subsubsection{Protein collection from cell culture and Western blotting}

Plated cells were washed three times in ice cold PBS and then lysed in RIPA buffer for 30 minutes on ice. Samples were spun at $14,000 \mathrm{~g}$ for 10 minutes and supernatants collected. Protein concentrations were calculated using the BioRad Protein Assay. Western blotting then proceeded as outlined in Fly Experiment section (section 3.1.10).

\subsubsection{Oxidative stress testing and measurement of cell viability}

Cells were incubated for 16 hours in the presence of either hydrogen peroxide (100 $\mu \mathrm{M}$ ) or rotenone in DMSO (HEK293: $200 \mu \mathrm{M}$, rat cortical: $1 \mu \mathrm{M}$ rotenone). Rotenone control cells were treated with equivalent amount of DMSO alone. After overnight oxidative stress treatment, cells were washed once in PBS, fixed in 4\% PFA for 10 minutes, washed twice in PBS and then permeabilized in $0.1 \%$ Triton X-100 in PBS for 10 minutes. After three PBS washes, the cells were incubated with Hoechst nuclear stain for 30 minutes, washed twice in PBS and imaged on a fluorescent microscope (Leica DMI6000B). Using a macro within the Leica Qwin V3 quantification software, cell number remaining in each well was assessed by 
counting total fluorescent nuclei (6 images per well at 10X, with minimum 6 wells per genotype in a 24-well culture dish).

\subsubsection{ATP synthesis assay}

Method was adapted from Gegg et al. ${ }^{214}$ Cells were trypsinized and washed three times in cold PBS. Cells were then re-suspended in incubation medium $\left(2 \times 10^{5} / \mathrm{ml}, 25 \mathrm{mM}\right.$

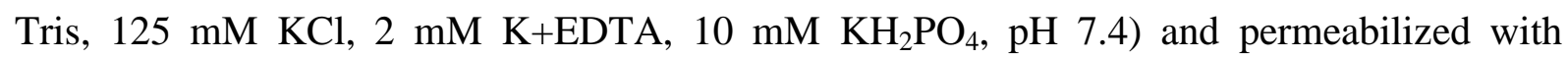
digitonin $(40 \mu \mathrm{g} / \mathrm{ml})$. To measure ATP produced via specific complexes, an aliquot of cells $\left(2 \times 10^{4}\right)$ (minimum of 12 replicates per experiment) was resuspended in a complex incubation medium supplemented with $1 \mathrm{mg} / \mathrm{ml} \mathrm{BSA}$ and $2 \mathrm{mM}$ ADP and for complex I, III, IV ATP production measurement: $10 \mathrm{mM}$ glutamate, $2 \mathrm{mM}$ malate; for complex II, III, IV: $20 \mathrm{mM}$ succinate, $80 \mu \mathrm{g} / \mathrm{ml}$ Rotenone; complex IV: $4 \mathrm{mM}$ ascorbate, $0.2 \mathrm{mM}$ nnntetramethyl, 20 $\mu \mathrm{g} / \mathrm{ml}$ Antimycin A. Cells were then incubated for 20 minutes at $37^{\circ} \mathrm{C}$. The reaction was stopped by addition of $6 \%$ perchloric acid and samples neutralized with $3 \mathrm{M} \mathrm{K}_{2} \mathrm{CO}_{3}$. ATP in each sample was measured using the CellTiter-Glo Luminescent reagent (Promega) in a Berthold Mithras LB940 following manufacturer- provided instructions. Total ATP levels were obtained using an ATP standard curve (ATP from Sigma), with final calculation expressing ATP as pmoles ATP per minute per 1 million cells. In the absence of substrates, production of ATP in the absence of substrates was less than $1 \%$ of values with substrates.

\subsubsection{Measurement of steady state cellular ATP}

HEK293 cells were trypsinized, counted and seeded $\left(2 \times 10^{4}\right.$ cells/ well $)(12$ replicates per experiment) in a opaque white 96 well plate (Nunc, Thermoelectron GmbH, Germany). Total ATP levels were measured using the CellTiter-Glo Luminescent reagent (Promega) in a Berthold Mithras LB940 (Berthold Technologies, Germany) plate reader following manufacturer provided instructions. Total ATP levels were obtained using an ATP standard curve (ATP from Sigma) and related to cell number seeded per well. 


\subsubsection{Assessment of mitochondrial membrane potential}

Following manufacturer provided instructions, cells ( 6 replicates per experiment) in a 96-well plate (black sided, clear bottom plate; Greiner Bio One, Frickenhausen, Germany)

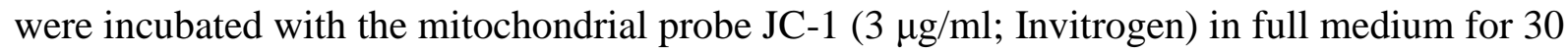
minutes at $37^{\circ} \mathrm{C}$. Following incubation, cells were washed twice in PBS before JC-1 mitochondrial aggregates were measured in a plate reader (Berthold Mithras LB940; excitation at $530 \mathrm{~nm}$, emission at $590 \mathrm{~nm}$ ). As a control, JC-1 fluorescence was measured in the presence of the mitochondrial membrane potential inhibitor CCCP (Carbonyl cyanide $m$ chlorophenylhydrazone, $50 \mu \mathrm{M})$. Protein content per well was quantified using the Bradford assay and total fluorescence expressed against protein.

\subsubsection{Immunocytochemistry}

Cells were plated on PLL-coated glass slips. Forty- eight hours after transfection, cells were washed once in PBS and then fixed in 4\% PFA for 10 minutes. After three washes in PBS (pH 7.4), cells were permeabilized in $0.1 \%$ Triton $\mathrm{X}-100$ in PBS for 10 minutes, followed by washing PBS. Cells were then blocked for one hour at RT in 1\% BSA in PBS. The primary antibody was incubated overnight at $4^{\circ} \mathrm{C}$ on a shaker with $1 \%$ BSA in PBS. Antibodies used: monoclonal rat anti- $\alpha$-Synuclein (1:500, Alexis Biochemicals/Enzo Life Sciences, Germany); monoclonal mouse anti- NeuN (1:500, Chemicon); monoclonal mouse anti-TRAP1 (1:300, Alexis Biochemicals). For visualization of mitochondria, cells were incubated with Mitotracker Orange CMTMRos (300 nM following manufacturer's instructions, Invitrogen) for 30 minutes at $37^{\circ} \mathrm{C}$ prior to fixation.

Following primary antibody incubation, cells were washed three times in RT PBS before incubation with respective secondary antibodies for one hour at RT in PBS (all secondary antibodies 1:1000, anti-mouse or rat AlexaFluor-488, 543, 633, Invitrogen). Cells were again washed three times in PBS before being mounted on glass slides (Fischer- 
Scientific) using Mowiol (Calbiochem, Germany), with or without the anti-bleaching agent

DABCO or nuclear stain Hoechst (Sigma-Aldrich). Staining was visualized using a Leica

DMI 6000B fluorescent microscope or the confocal microscope (Leica DM IRE2).

\subsubsection{Immunoprecipitation (IP)}

Protein was collected from HEK293 cells over-expressing $\alpha$-Synuclein alone or both $\alpha$-Synuclein and TRAP1 using the above described method for protein isolation (section 3.2.5.) and quantification from cell culture. IP procedure followed the general procedure supplied with the Protein A Agarose (Roche) beads. To reduce background due to unspecific binding, protein samples were first pre-incubated with Protein A Agarose beads for 4 hours at $4^{\circ} \mathrm{C}$. After removal of the agarose beads by centrifugation, protein samples were incubated overnight at $4{ }^{\circ} \mathrm{C}$ with either mouse anti- $\alpha$-Synuclein monoclonal (Cell Signaling) or mouse anti-TRAP1 monoclonal (BD Biosystems) antibody. Incubation of samples with rabbit antiGFP polyclonal served as a negative control. After overnight incubation, Protein A agarose beads were added to the samples and incubated for 2 hours at $4^{\circ} \mathrm{C}$. After incubation, the agarose-antibody-antigen complexes were collected by centrifugation and washed three times in ice cold RIPA buffer before running the samples on polyacrylamide gels and proceeding with the above described Western Blotting protocol (section 3.1.10.).

\subsubsection{Mitochondrial isolation}

Mitochondria were isolated from HEK293 cells transfected with $\alpha$-Synuclein[A53T] using a mitochondrial isolation kit (Sigma MITOISO2) following manufacturer provided instructions. Purification of mitochondria was assessed using Western blotting for the following mitochondrial association proteins: VDAC1 (porin1; outer membrane protein), Cytochrome c (inner mitochondrial membrane protein) and TRAP1 (inner mitochondrial membrane space).

\subsection{List of basic laboratory buffers}


Listed below are all basic laboratory buffers used:

\subsubsection{DNA extraction and electrophoresis}

PBS: $0.137 \mathrm{M} \mathrm{NaCl}, 8.2 \mathrm{mM} \mathrm{Na}_{2} \mathrm{HPO}_{4}, 1.5 \mathrm{mM} \mathrm{KH}_{2} \mathrm{PO}_{4}, 0.2 \mathrm{mM} \mathrm{KCl}, \mathrm{pH} 7.4$

TAE buffer: 40 mM Tris/acetate; 1mM EDTA; pH 8.0

6x TAE loading dye: $30 \%(\mathrm{v} / \mathrm{v})$ glycerol, $0.25 \%(\mathrm{w} / \mathrm{v})$ bromophenol blue, $0.25 \%(\mathrm{w} / \mathrm{v})$, xylenol cyanol

Agarose gel: $0.5 \%$ - 2\% agarose in TAE, $0.001 \%$ ethidium-bromide

\subsubsection{Protein extraction and Western blotting}

RIPA buffer: $50 \mathrm{mM}$ Tris, $\mathrm{pH} 8.0,0.15 \mathrm{M} \mathrm{NaCl}, 0.1 \%$ SDS, $1.0 \% \mathrm{NP}-40,0.5 \% \mathrm{Na}-$ Deoxycholate, 2 mM EDTA, Complete ${ }^{\mathrm{TM}}$ Protease Inhibitors (Roche Applied Sciences), $\mathrm{pH}$ 7.4

5x Laemmli buffer: $10 \%$ glycerol, 2\% SDS, 12.5\% $0.5 \mathrm{M}$ Tris-Cl, $\mathrm{pH} \quad 6.8,5 \% \quad \beta-$ mercaptoethanol, $0.05 \%$ bromophenol blue

Running Buffer: 25 mM Tris/HCl; $0.192 \mathrm{M}$ glycine; 0.2\% SDS, pH 8.3

Transfer buffer: 25 mM Tris, 192 mM glycine, $20 \%$ methanol, pH 8.3

Ponceau solution: $0.1 \%$ Ponceau-S in $5 \%$ acetic acid

TBS: $10 \mathrm{mM}$ Tris/HCl, $150 \mathrm{mM} \mathrm{NaCl}, \mathrm{pH} 7.5$

TBS-T: TBS with $0.1 \%(\mathrm{v} / \mathrm{v})$ Tween 20

Blocking solution: $5 \%(\mathrm{w} / \mathrm{v})$ skim milk in TBS

Stripping buffer: $0.2 \mathrm{M}$ glycine, $0.5 \mathrm{M} \mathrm{NaCl}, \mathrm{pH} 2.8$

\subsubsection{Bacterial transformation and cloning}

LB-medium: $10 \mathrm{~g} / \mathrm{l}$ tryptone, $5 \mathrm{~g} / \mathrm{l}$ yeast extract, $5 \mathrm{~g} / \mathrm{l} \mathrm{NaCl}$

LB-plate: $2 \%(\mathrm{~m} / \mathrm{v})$ agar in LB-medium

SOC: $20 \mathrm{~g} / \mathrm{l}$ tryptone, $5 \mathrm{~g} / \mathrm{l}$ yeast extract, $10 \mathrm{mM} \mathrm{NaCl}, 10 \mathrm{mM} \mathrm{MgCl} 2,10 \mathrm{mM} \mathrm{MgSO} 4,20$ $\mathrm{mM}$ glucose 


\subsubsection{Staining of cell culture slides for histology}

PBS: $137 \mathrm{mM} \mathrm{NaCl}, 8.2 \mathrm{mM} \mathrm{Na} 2 \mathrm{HPO}_{4}, 1.5 \mathrm{mM} \mathrm{KH}{ }_{2} \mathrm{PO}_{4}, 2.7 \mathrm{mM} \mathrm{KCl}, \mathrm{pH} 7.4$

TBS: $0.1 \mathrm{M}$ Tris, $150 \mathrm{mM} \mathrm{NaCl}, \mathrm{pH} 7.4$

Mowiol-mounting medium: 24\% w/v glycerol, $0.1 \mathrm{M}$ Tris-base $\mathrm{pH} 8.5,9.6 \%$ w/v Mowiol $4.88,2.5 \%$ w/o DABCO

\subsection{Statistics}

Data was analyzed using GraphPad Prism 4.0 (GraphPad Software Inc., La Jolla, USA), using 1-way ANOVA followed by Newman-Keuls post testing when significant $(\mathrm{p}<0.05)$, unless otherwise noted. Use of a 2-way ANOVA was noted in the text. Survival data was analyzed with the Kaplan-Meier analysis method and the Log Rank Test for curve statistical comparison analysis. Statistical significance referred to as: ${ }^{*}, \mathrm{p}<0.05 ; * *, \mathrm{p}<0.01$ and $* * *, \mathrm{p}<0.001$. All data is presented as mean \pm SEM. 


\section{Results.}

\subsection{Initial Experiments: Fly Model Establishment and Genetic Screening Parameter Determination}

\subsubsection{Generation of recombined ddc $>$ and elav $>\alpha$-Synuclein [A53T]-expressing}

flies

After creating recombined flies (ddc and elav>A53T) (breeding schema, Methods 3.1.1.), human $\alpha$-Synuclein[A53T] expression in flies was confirmed using Western blotting (Fig. IV-1).

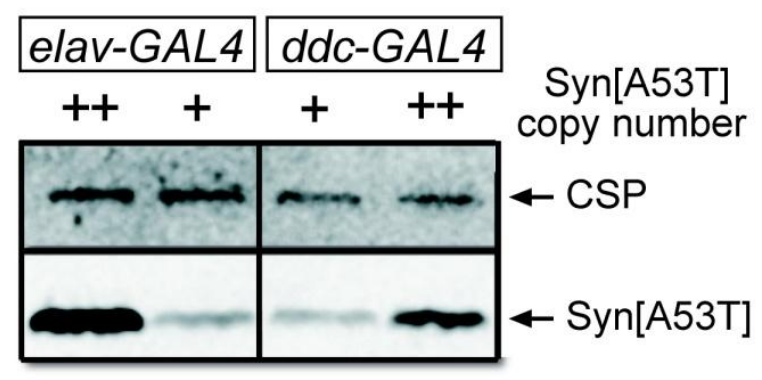

Fig. IV-1. Expression of human $\alpha-S y n u c l e i n[A 53 T]$ in fly brains in either aminergic neurons (ddc-GAL4 driver) or pan-neuronally (elav-GAL4 driver). Expression of $\alpha$-Synuclein[A53T] (16 kDa) was compared to CSP- $\alpha$ (protein loading control, $34 \mathrm{kDa}$ ). Lanes from left to right: 1) elav>A53T/ elav >A53T, 2) elav>A53T/+, 3) ddc $>$ A53T/ + , 4) ddc $>$ A53T/ ddc $>\alpha$-SynA53T. For all Western blots: $\alpha$-Synuclein[A53T] represented by 'Syn[A53T]' and CSP- $\alpha$ as 'CSP'. Representative blot of 3 experiments.

\subsubsection{Aging of $\alpha$-Synuclein[A53T]-expressing flies}

To investigate whether expression of a neurotoxic protein had effects on overall viability, recombined flies either homo- or heterozygous for $\alpha$-Synuclein[A53T] gene (ddc or elav-GAL4 driver expression) were aged. Longevity was compared to control flies. Life span of flies expressing one copy of $\alpha$-Synuclein[A53T] was not different than control, while flies expressing two copies of $\alpha$-Synuclein[A53T] showed a reduced longevity independent of driver used (Fig. IV-2). For screening purposes however, the ddc driver was chosen, as the specific expression of $\alpha$-Synuclein[A53T] within aminergic (serotinergic and dopaminergic) neurons. Expression in aminergic neurons more closely replicates the toxicity seen in PD. 

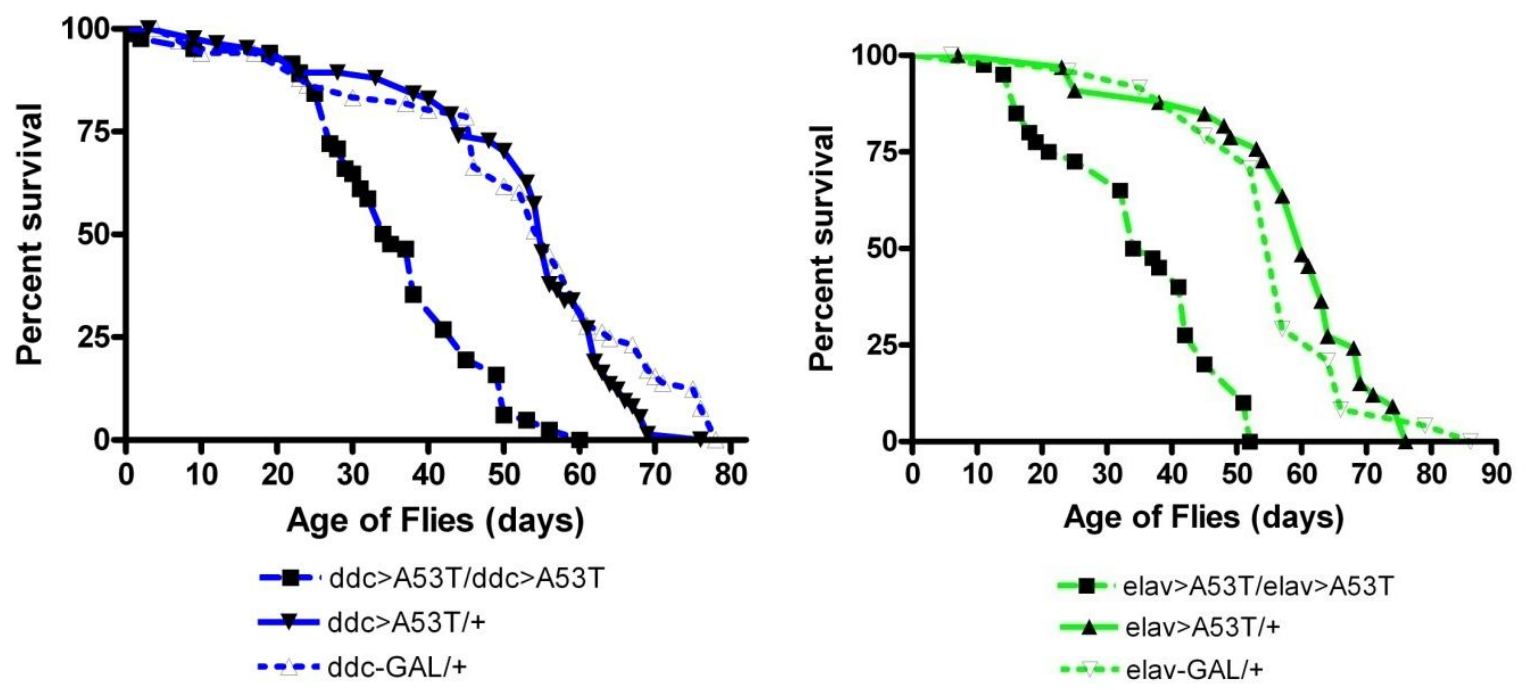

Fig. IV-2. Longevity of flies expressing $\alpha$-Synuclein[A53T] under either ddc or elav-GAL4 driver control. Flies either homo or heterozygous for $\alpha$-Synuclein[A53T] were aged and longevity compared to Controls (driver/+). Flies expressing 2 copies of gene show significantly decreased survival (***, p<0.0001 vs. Controls under either elav or ddc-GAL4 driver) ( $n=50-70$ for double copy flies, otherwise $n<100)$.

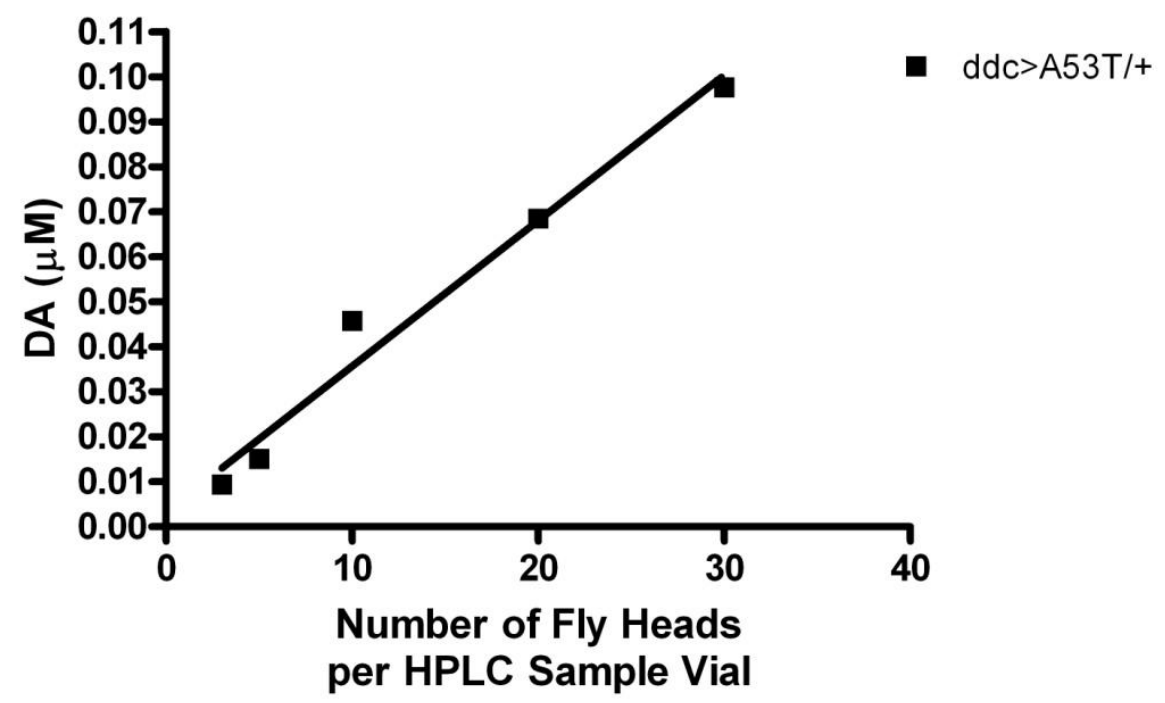

Fig. IV-3. Sensitivity of HPLC to measure fly head DA. Number of fly heads per measurement vial was varied (from 3-30) and absolute DA amounts measured using HPLC analyzed via linear regression. Linear regression $r^{2}=0.997(n=3)$.

\subsubsection{Determination of appropriate sample size number for HPLC fly brain DA measurements}

To determine the amount of fly head DA reproducibly measurable using the HPLC, number of fly heads per sample was varied and HPLC values compared (day $0 \mathrm{ddc}>\mathrm{A} 53 \mathrm{~T} /+$ flies). A linear relationship between DA measured and number of fly heads per sample was 
observed (Fig. IV-3). Thus, to minimize both number of flies required and strain on the HPLC system resulting from accumulated biomass, three fly heads for one HPLC sample was decided to be the standard method for measuring fly head DA.

\subsubsection{Influence of time of day on DA content in fly heads}

DA is a neurotransmitter that exhibits circadian fluctuations in synthesis rates and receptor sensitivity. ${ }^{215}$ Thus, the measurable level of DA in fly brains across time of day was investigated, for the purpose of determining whether or not time of fly collection could influence screening results. A slight variation was observed in fly brain DA amount with time of day (Fig. IV-4). It was thus determined that all screening sample collection would be done at a fixed time point of $12-1 \mathrm{pm}$, to minimize variation due to time.

\subsubsection{Variation in DA content between fly lines}

To check for variation in absolute DA level dependent on genetic background, freshly hatched flies were collected from three different fly lines and DA measured (Fig. IV-5). A difference between elav and ddc-GAL4 driver lines vs. white flies was observed, suggesting an influence of genetic background on absolute DA levels.

\subsubsection{Measurement of fly head DA over time following tyrosine hydroxylase (TH) inhibition ( $\alpha$-methyl tyrosine (MT) treatment)}

To confirm that HPLC had both the required sensitivity to measure decreasing fly head DA and the specificity of DA measurement, control flies were treated with $\alpha$-MT. $\alpha$-MT inhibits TH, the enzyme catalyzing DA production. After fly collection from 0-8 days of age, a dose- dependent decrease in DA was observed, confirming that HPLC had both specificity and sensitivity to measure decreasing levels of fly brain DA (Fig. IV-6).

\subsubsection{Measurement of $\mathrm{DA}$ with age in control and $\alpha$-Synuclein[A53T]- expressing flies}


The effect of age on DA levels was investigated by collecting DA samples at 1 week time intervals ( 0 to 5 weeks of age) for control and ddc>A53T flies. Evident from three

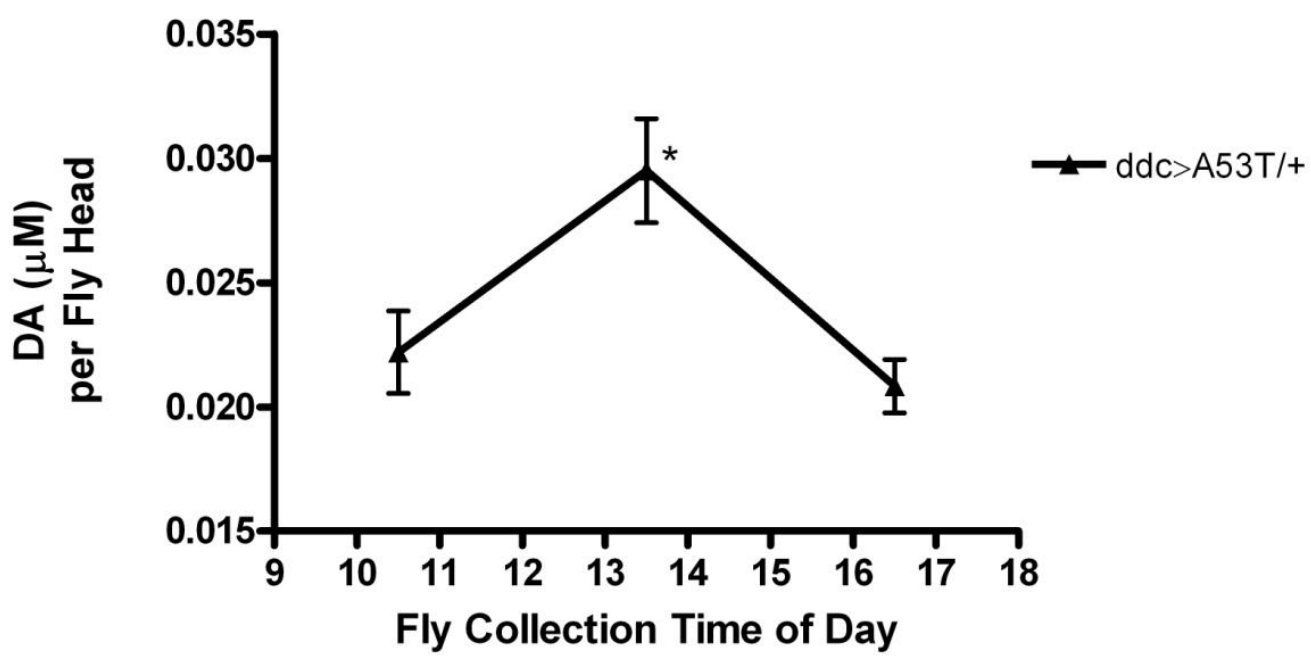

Fig. IV-4. Changes in fly head DA dependent on time of day. Fly heads collected at indicated time of day were analyzed for DA using HPLC. Significant difference (1-way ANOVA) between time points noted: *, $\mathrm{p}<0.05$ vs. 1030 and $1630(\mathrm{n}=3)$.

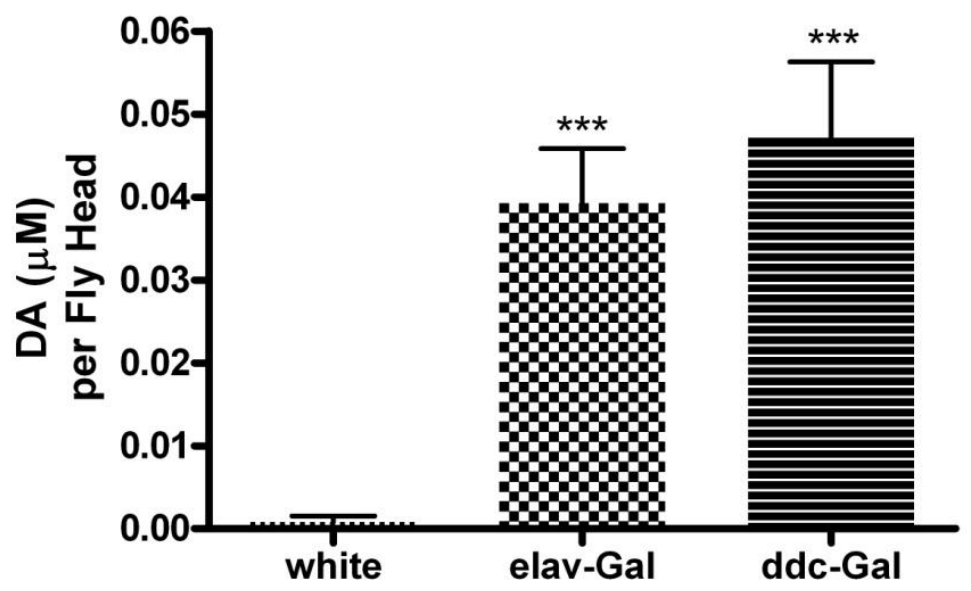

Fig. IV-5. DA head content from flies with different genetic background. Flies were collected directly after eclosion. DA from head lysates was measured using HPLC. Genotypes analyzed: driver line/+ and white $\left(\mathrm{w}^{118}\right)$ flies. Significant differences (1-way ANOVA) observed: ***, $\mathrm{p}<0.0001$ vs. white $(\mathrm{n}=3)$.

weeks of age, there was a marked decreased in DA in ddc>A53T flies (Fig.IV-7). Based on this time course, screening collection time points of 1 and 4 weeks of age were chosen. Each screen sample would thus be presented as a change in DA (\%), rather than absolute value of DA. Using the 1 and 4 week collection time points, DA head content was measured from both elav>A53T/+ flies and additional controls (Oregon R wild type flies, UAS-A53T alone, LacZ 
or GFP expression under ddc or elav-GAL4 driver). Expression of $\alpha$-Synuclein[A53T] under elav-GAL4 expression further enhances loss of DA vs. ddc-GAL4 driver. Age-dependent loss of DA is specific to $\alpha$-Synuclein[A53T] expression, as flies expressing either

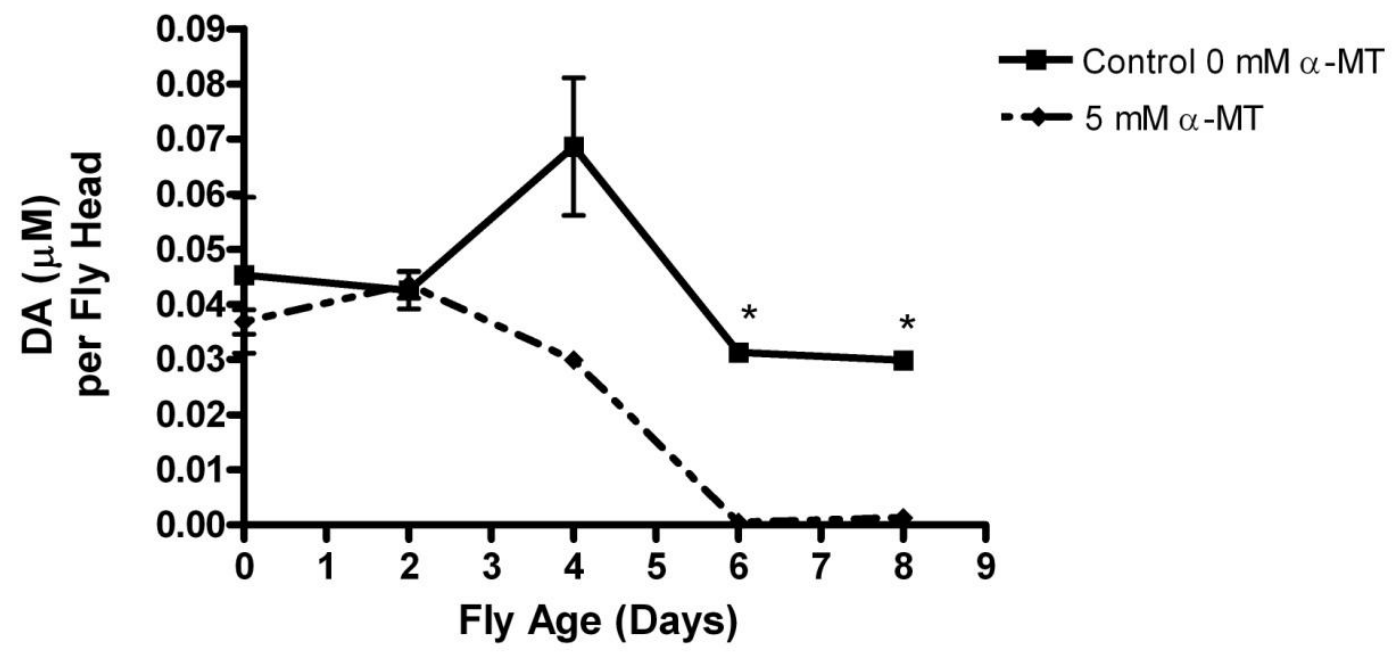

Fig. IV-6. Dose- dependent reduction in fly head DA upon treatment with TH inhibitor, a-MT, as measured by HPLC. Wt flies were collected at eclosion and treated daily with $\alpha$-MT. Flies were collected at 2 day intervals for measurement of head DA using HPLC: Significant differences (t-test): *, p<0.05 for Control (untreated) vs. $5 \mathrm{mM} \alpha-\mathrm{MT} . \mathrm{n}=3$.

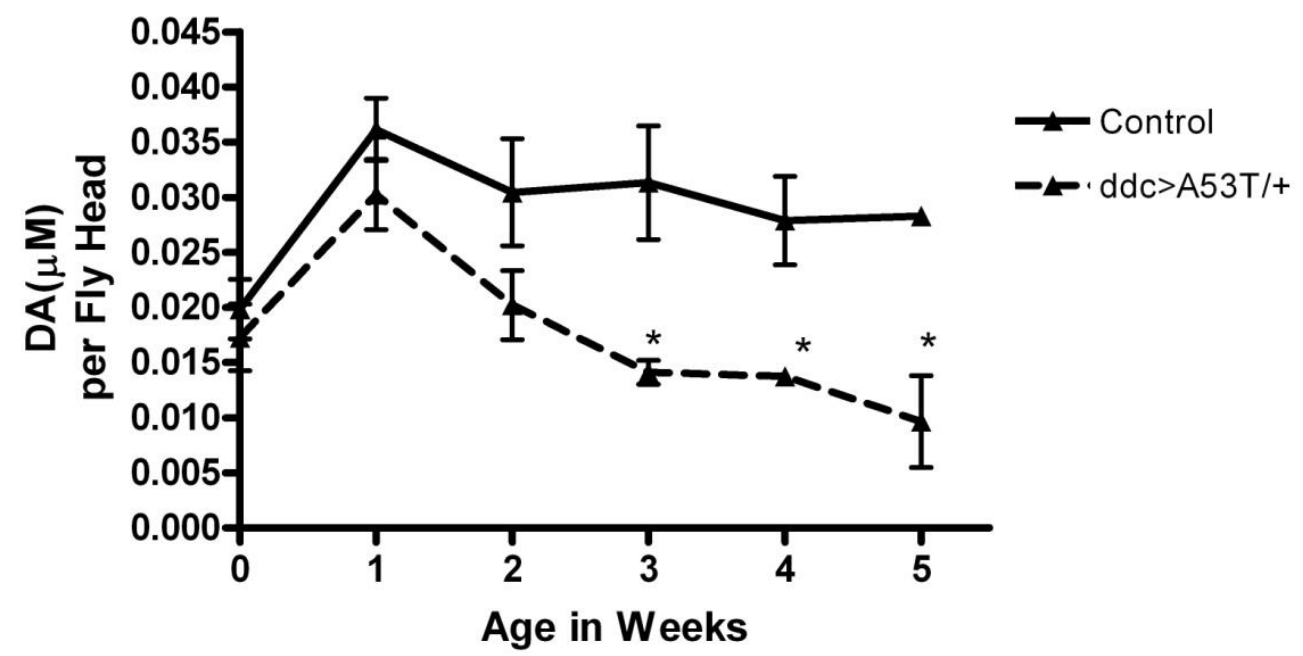

Fig. IV-7. Aging-dependent loss of DA in control and ddc $>$ A53T/ +flies. Control (ddc-GAL/+) and $\alpha-$ Synuclein[A53T]-expressing flies were collected at 1 week intervals and heads assessed for DA content using HPLC. Significant differences (t-test) at each time point observed:*, $\mathrm{p}<0.05$ vs. Control (ddc-GAL/+). $n=10-15$ for age 1 and 4 weeks, $n=3$ for other points.

LacZ/GFP did not show pronounced aging-dependent loss of DA (Fig. IV-8).

In addition, several genes previously related to PD were also tested for altering DA content (Parkin, PINK1 and HTRA2). As expected, both putative Parkin and HTRA2 
deficiency caused a decrease in DA content at 4 weeks, consistent with their posited protective effects, acting to confirm sensitivity of the screen for valid modifiers (see Fig. IV-8 and Appendix Results, Table A2-3). ${ }^{189,} 195$ Unexpected was the result that RNAi line for PINK1 did not result in an enhanced loss of DA. However this result can most likely be explained by an ineffective RNAi. Previously published phenotypes based upon RNAimediated silencing of PINK1 of mitochondrial morphology, infertility, oxidative stress sensitivity and early mortality of flies could not be reproduced. ${ }^{216} 217$ However these papers utilized another RNAi line and thus it seems possible that this is the reason for the lack of DA change. In addition, although it was not found in a 'low' deficiency line, an RNAi line for silencing of thioredoxin reductase-1 (VD47307), the Drosophila equivalent to human glutathione-S reductase, a key antioxidant enzyme, was bred with $\alpha$-Synuclein[A53T]. As would be expected, there was a slight enhancement of DA loss with time. ${ }^{218}$ The flies also showed enhanced sensitivity to sensitive to paraquat under elav-GAL4 control. This serves as an additional positive confirmation of screen sensitivity.

\subsubsection{Loss of dopaminergic neurons in flies expressing $\alpha$-Synuclein[A53T]}

Although several previous studies utilizing Drosophila expressing $\alpha$-Synuclein have shown an aging-dependent loss of DA neurons, this has not been a consistent finding, as several studies using similar flies did not observe neuronal loss. ${ }^{135,142,219-222}$ Thus, it seemed pertinent to examine the extent of $\alpha$-Synuclein[A53T] toxicity in the present model system. Flies expressing GFP within dopaminergic neurons (ddc $>$ GFP) were crossed to flies containing transgenes for either LacZ (control) or $\alpha$ - Synuclein[A53T]. The high colocalization of neurons stained with a TH antibody and the GFP signal indicated a rather specific TH-neuron-specific expression with the ddc-GAL4 driver (Fig. IV-9). 


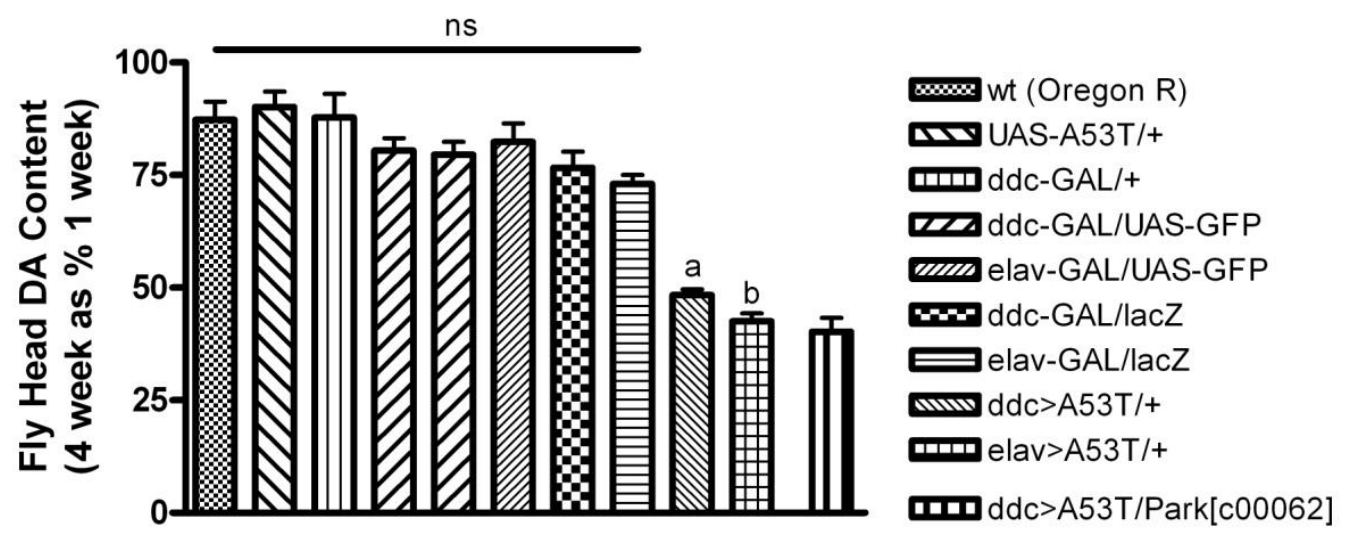

Fig. IV-8. Fly head DA content with time (4 week as \% 1 week value). Flies of both control genotypes and those expressing $\alpha$-Synuclein[A53T] were collected at 1 and 4 weeks of age for head DA measurement using HPLC. Significant differences (1-way ANOVA) observed: $a, b: p<0.001$ vs. respective control of ddc-GAL/+ or elav-GAL/+. All other differences ns. As a positive control for screen ability to pick up modifiers, genes previously connected to PD were screened for enhancing loss of DA in combination with $\alpha$-Synuclein[A53T] (putative Parkin disruption allele (Park[c00062], BL10006) in combination with ddc>A53T). n=3 for ddc $>$ A53T/Park[c00062] flies, $n=6$ for all other controls, $n=10$ for all other $\alpha$-Synuclein[A53T] expressing flies.

GFP-expressing neurons within the major dopaminergic neuron clusters were then counted in

flies at 6 weeks of age. Flies expressing $\alpha$-Synuclein[A53T] showed a significant loss of neurons in the PPL1 and PPM1 clusters, a result confirming previously published reports of neuron loss in these clusters (Fig. IV-10).

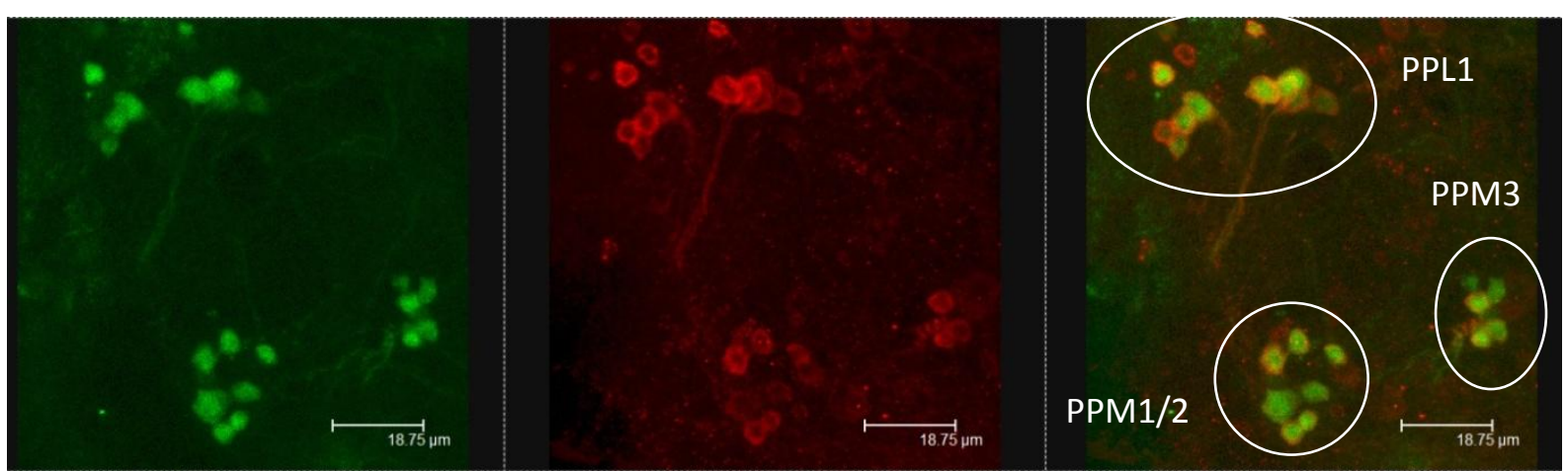

Fig. IV-9. Ddc-GAL4 driven GFP co-localized in TH-stained fly brain neurons. Confocal section of a fly brain expressing GFP (green, left) under control of ddc-GAL4 was stained with anti-TH antibody (Cy3 secondary, red, middle). Both signals show a high degree of overlap (merge, right). Picture from optical slice in confocal Z-stack. 


\section{RESULTS}

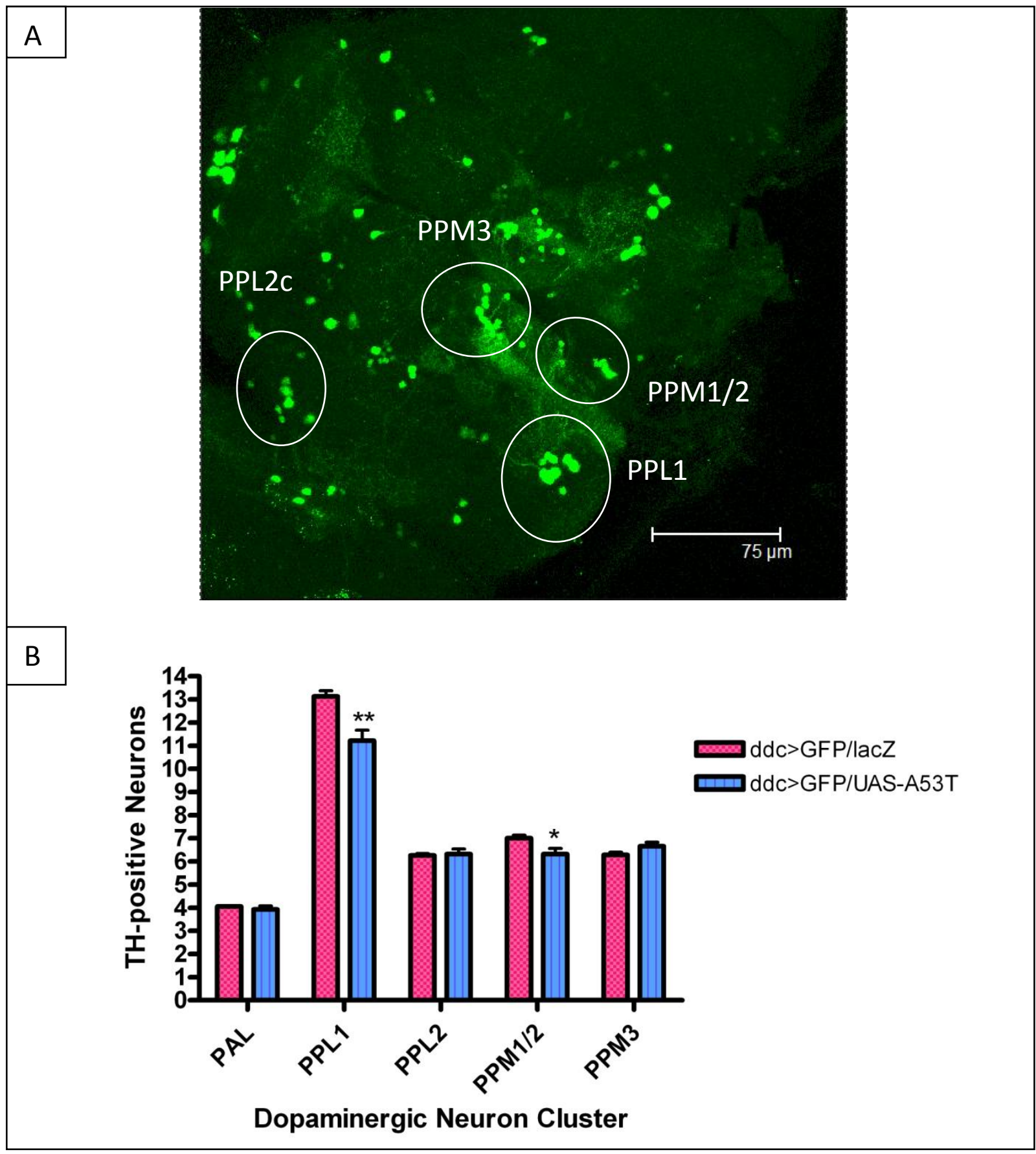

Fig. IV-10. Fly brain dopaminergic neuron count at 4 weeks of age. A: Confocal Z stack of brain derived from ddc $>$ GFP expressing fly. Circles indicating approximate dopaminergic neuron cluster locations. B: Neuron count from respective neuron clusters of ddc $>$ GFP/UAS-A53T-expressing flies at 4 weeks of age compared to control (ddc $>$ lacZ). Significant differences (t-test for specific cluster data) observed: **, $\mathrm{p}<0.01, *, \mathrm{p}<0.05$ vs. ddc $>$ GFP/lacZ $(n>15)$. 


\subsubsection{Data Summary from Initial Experiments to Establish the Model}

- Flies generated: ddc $>$ A53T and elav>A53T

- Flies with 2 copies of $\alpha$-Synuclein[A53T] show reduced lifespan

- Sensitivity of HPLC sufficient to measure 3 fly heads per sample

- Age-dependent loss of brain DA observed in flies expressing $\alpha$ Synuclein[A53T]

- Time point for fly collection for deficiency screen: 1 and 4 weeks

- Loss of TH neurons in PPL1, PPM1 DA clusters observed for flies expressing $\alpha$-Synuclein[A53T] under ddc-GAL4 driver

\subsection{Primary Deficiency Screen Data (HPLC measurement of fly head DA)}

To screen for modification in $\alpha$-Synuclein[A53T] neurotoxicity, loss of DA with time was used as an endpoint. Flies from the 'Bloomington Deficiency Kit' (270 lines, each containing a defined deletion) were crossed to ddc $>$ A53T flies. Offspring negative for balancer chromosome phenotypic markers were collected at 1 and 4 weeks of age and levels of DA in heads of respective flies measured. An overall summary of the primary data can be seen in Fig. IV-11. Individual HPLC values for each line can be seen in Appendix Results Table A2-1.

Results of the initial screen were categorized on the basis of 'low' (less than $50 \%$ DA at 4 weeks), 'high' (greater than 100\% DA at 4 weeks), 'reduced viability' (offspring did not live to 4 week collection point) and lethal (crosses without viable offspring). Identified deficiency lines were then rebred for purpose of confirmation. After rebreeding, 24 lines were confirmed to result in 'low' DA at 4 weeks vs. 1 week (Fig. IV-12) and 52 lines were designated as resulting in 'high' DA. Five crosses resulted in lethality and nine crosses were semi-lethal (Table IV-1) (full results of screen in Table A2-I, Appendix Results): 


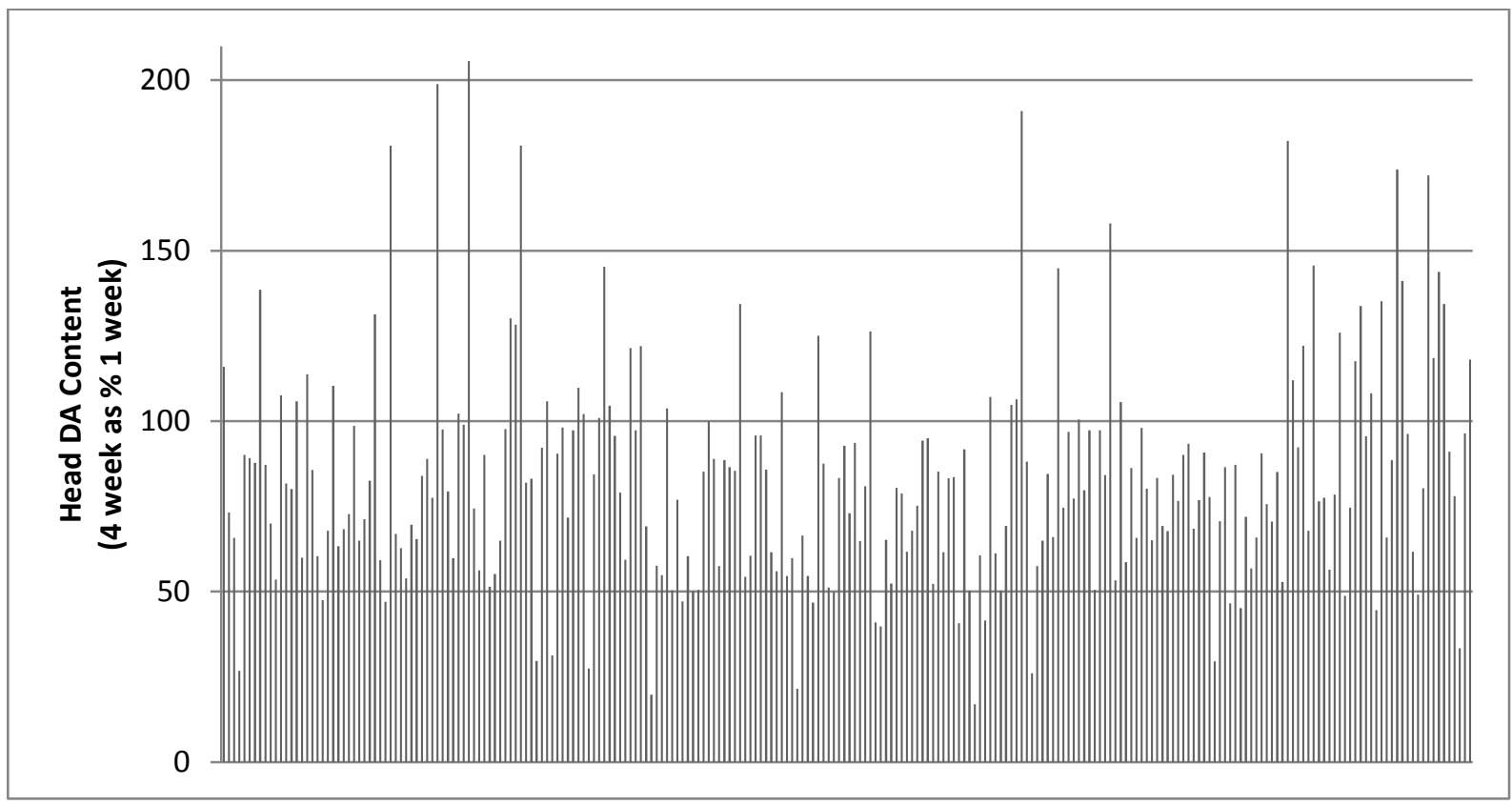

Fig. IV-11. Change in fly head DA with time ( 4 week as $\% 1$ week) for deficiency cross offspring (ddc> A53T X deficiency line).

The numerous crosses resulting in 'high' DA most likely resulted from an alteration in the 1 week peak of DA, alongside possible deletion of enhancer genes. However the 'low' group is easier to interpret, as large losses to DA content were not observed in control flies and thus more likely relates to deletion of suppressor genes. Thus, the decision was made to concentrate on the 'low' and lethal candidate lines, as results more unambiguous. Investigation into the 'high' group could be pursued at a future date.

The cross resulting in the greatest loss of DA was Bloomington line 1007 (19.87\% of 1 week DA at 4 weeks). This deficiency contains 158 genes, which were examined for any possible 'obvious' candidates to use as a beginning for screening individual gene crosses for loss of DA (using P-element gene disruption or RNAi lines for each gene crossed to ddc $>\alpha-$ Synuclein[A53T]). TRAP1, a mitochondrial chaperone, had been recently identified as downstream phosphorylation target of the PD protein PINK1. ${ }^{103}$ Without TRAP1 phosphorylation, PINK1's protective effect was reduced. TRAPl was located within deficiency 1007 and thus a hypothesis implicating a decrease in TRAP1 enhancing $\alpha$ Synuclein[A53T] toxicity in Drosophila was suggested. The fly gene's functional domains 
(HSP-90 like and a histidine kinase-like ATPase) share high homology with the human gene and thus a conservation of function seemed likely. ${ }^{223}$ Several lines publicly available, potentially interfering with TRAP1 expression were: a gene disruption line for TRAP1 (TRAP1[KG06242]; BL14032) and 2 RNAi lines from NIG-FLY (TRAP1-RNAi[3152R1] and TRAP1-RNAi[3152R1]; NI3152R1 and NI3152R3). ${ }^{224}$ Alteration of TRAP1 expression with these lines in combination with both $\mathrm{ddc}>\mathrm{A} 53 \mathrm{~T}$ and elav $>$ A53T was first examined. A decrease in TRAP1 was observed using TRAP1[KG06242] in combination with ddc $>$ A53T and the greatest, most consistent decrease TRAP1 expression in elav>A53T was seen with

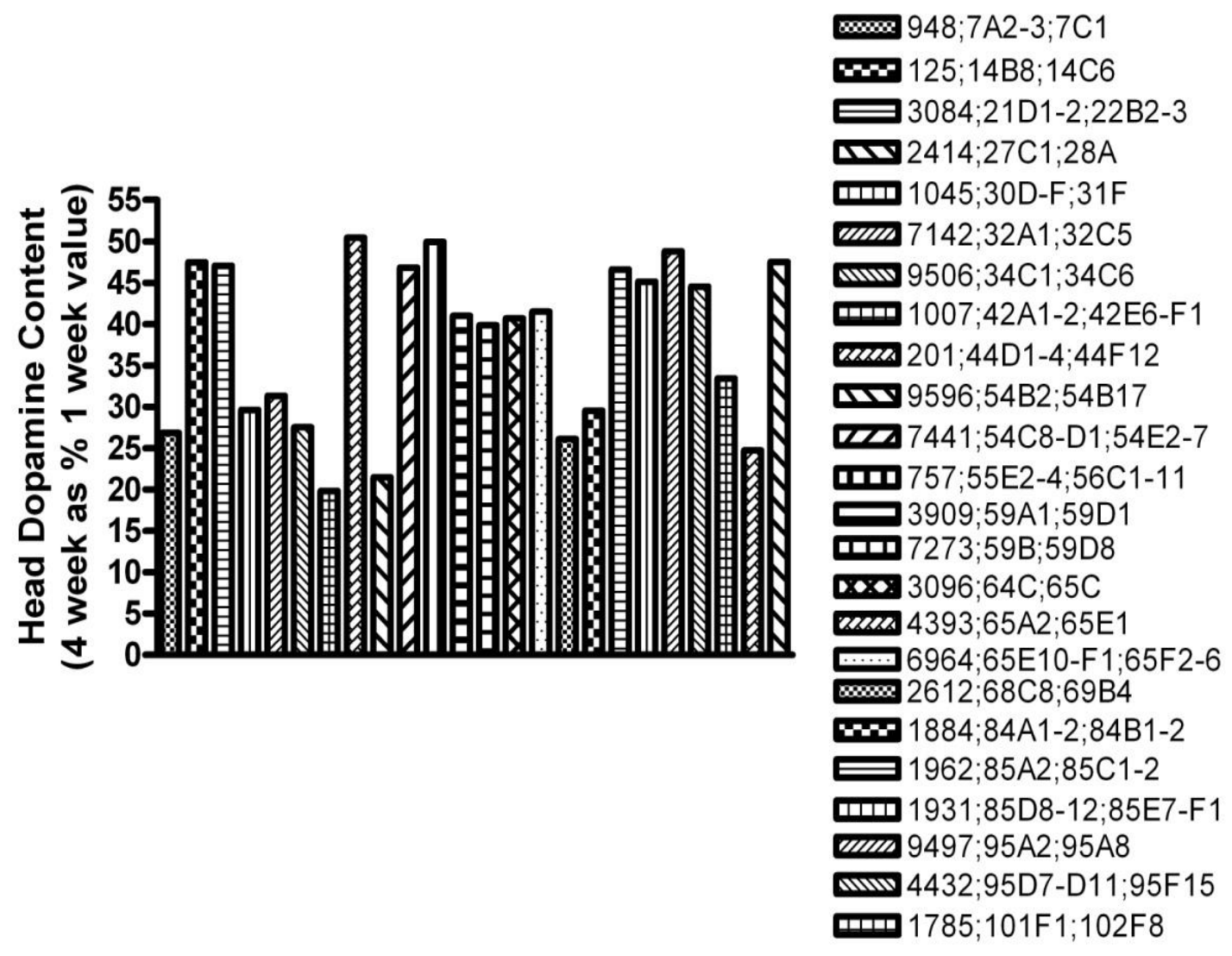

Fiv. IV-12. Twenty-four confirmed crosses (ddc>A53T/deficiency) resulting in 'low' (less than $50 \%$ of 1 week DA at 4 weeks) DA measurement. Legend numbers refer to Bloomington line number and region deleted by respective deficiency line (bars are means of 2 HPLC measurements). 


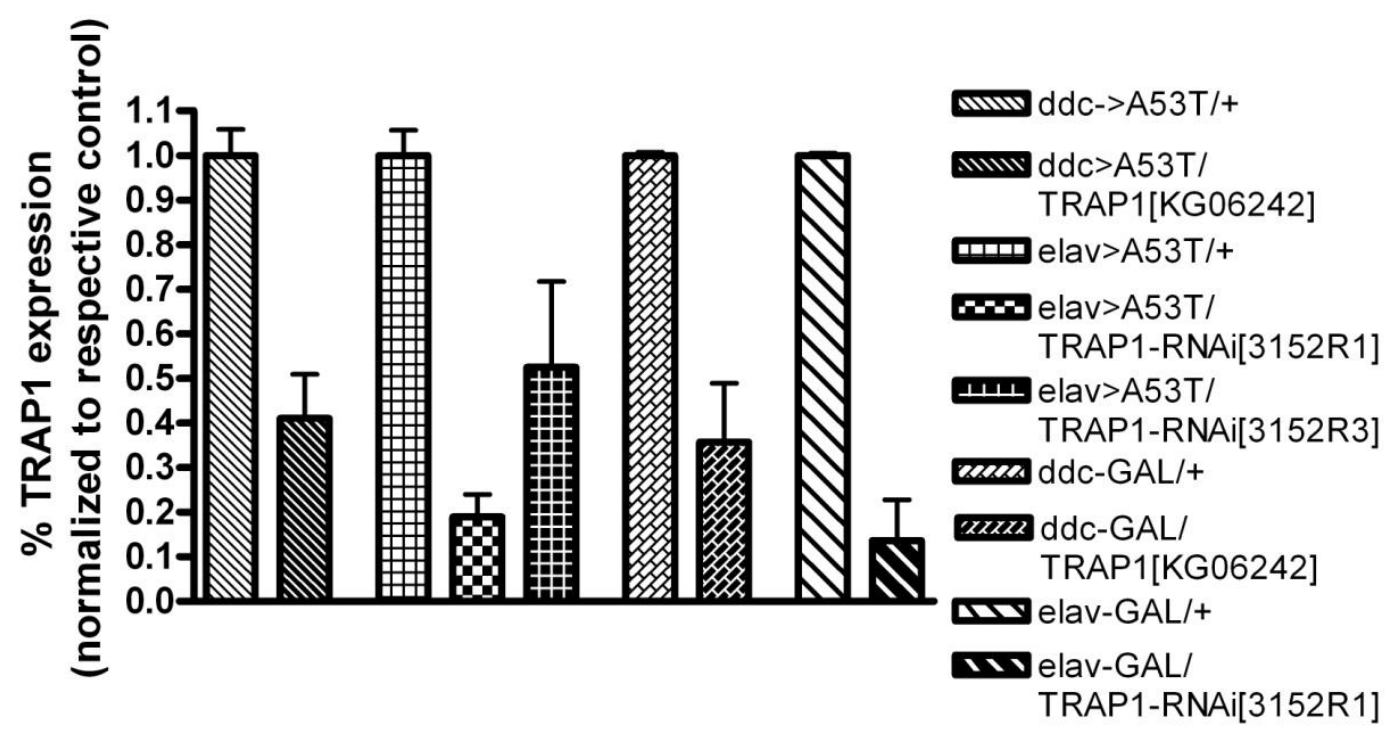

Fig. IV-13. Reduction of TRAP1 expression in fly brains with either ddc or elav-GAL4 $\alpha$-Synuclein[A53T] expression. Real time PCR was used to assess expression of TRAP1 expression in fly brains and demonstrated that use of either targeted P-element gene disruption or RNA interference alleles reduced TRAP1 expression. TRAP1 expression in flies of interest was normalized to TRAP1 expression in: ddc $>$ A53T/ + for ddc $>$ A53T/TRAP1[KG06242]; elav $>$ A53T/+ for elav $>$ A53T/TRAP1-RNAi[3152R1], elav $>$ A53T/TRAP1RNAi[3152R3]; ddc-GAL/+ for ddc-GAL/TRAP1[KG06242]; elav-GAL/+ for elav-GAL/TRAP1RNAi[3152R1] (n=3-4).

use of TRAP1-RNAi[3152R1] and this RNAi was thereafter used exclusively (Fig. IV-13).

Only real time PCR could be used to investigate down regulation of Drosophila TRAP1, because no Western Drosophila TRAP1 antibody is currently available. Thus, flies expressing either ddc $>$ A53T or elav $>$ A53T were then bred to either gene disruption or RNAi lines specific for TRAP1 and assessed for loss of brain DA. Decreased expression of endogenous TRAP1 expression caused an enhanced loss of 4 week DA in ddc $>$ A53T and elav $>$ A53T flies (Fig. IV-16). Given TRAP1's previous connection to PD-related processes, exploration of possible mechanism for modification to $\alpha$-Synuclein toxicity was then focused on TRAP1, with utilization of the above mentioned fly lines. Results from the investigation into TRAP1 and $\alpha$-Synuclein[A53T] continue in Results section 4.3.

\begin{tabular}{|l|l|l|}
\hline \multicolumn{3}{|l|}{ Table IV- I. List of Deficiency Lines Resulting in Lethality when crossed with ddc $>$ A53T } \\
\hline BL line & Deficiency Symbol & Deleted Segment \\
\hline 5281 & Df(1)dx81 & 5C3-10;6C3-12 \\
\hline 4953 & Df(1)BK10 & 15 F2-15F9;16C7-16C10 \\
\hline 7497 & Df(2L)Exel6011 & $25 \mathrm{C} 8 ; 25 \mathrm{D} 5$ \\
\hline
\end{tabular}




\begin{tabular}{|l|l|l|}
\hline 3024 & Df(3L)h-i22 & 66D10-11;66E1-2 \\
\hline 3340 & Df(3R)e-R1 & $93 \mathrm{~B} 6-7 ; 93 \mathrm{D} 2$ \\
\hline
\end{tabular}

Deficiency lines resulting in lethality were bred with driver line alone (negative for lethality), and then genes within the deficiencies were individually screened (using P-element disruption and RNAi lines) for lethality in combination with ddc $>$ A53T (full screening list, Appendix Results, Table A2-2). Only one gene disruption or RNAi line was found to result in lethality when in combination with ddc>A53T: gene disruption line for $K d n$ (BL 14436; CG3861, citrate synthase (CS)). Thus, most likely reason for the other instances of lethality was due to multiple gene product deletion, not connectable to any one gene. Use of the RNAi line for $k d n$ (VD26301) was semi-lethal when crossed to elav>A53T flies. CS is the first enzyme of the mitochondrial Krebs Cycle. Reduction of CS activity in flies with elav>A53T/26301 was confirmed with enzymatic assay (Fig. IV-14). However, given the promising data concerning TRAP1 and $\alpha$-Synuclein[A53T] at the time, the $k d n$ effect was not further investigated.

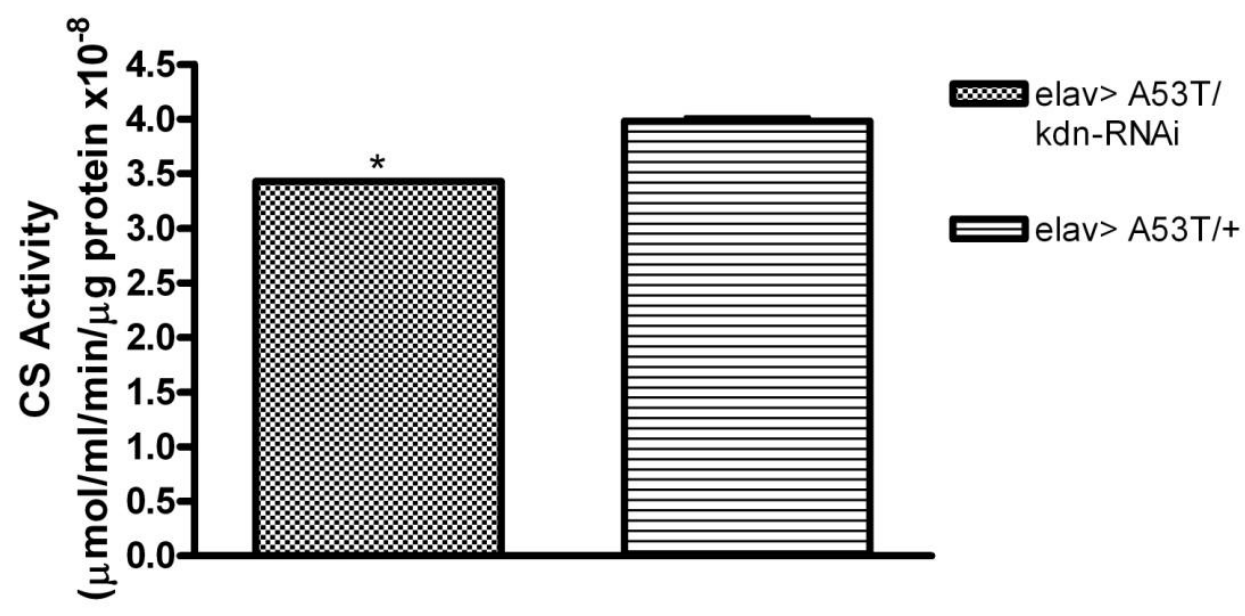

Fig. IV-14. CS activity reduction in flies elav $>$ A53T \pm RNAi for $\mathbf{C S}$. One week old flies were collected and freshly prepared brains assessed for CS activity. Significant difference (t-test) observed in CS activity between flies with pan-neuronal expression of kdn RNAi (VD26301) vs. flies without RNAi (*, p<0.05)(n=3). 


\subsubsection{Data Summary from Primary Deficiency Screen}

- ddc>A53T crossed to 270 deficiency lines, each containing a specific chromosomal deletion

- Brain DA measured at 1 and 4 weeks of age

- 24 crosses classified as 'low' (less than 50\% DA at 4 weeks age)

- Individual genes examined in lowest deficiency cross and reduction in TRAP1 identified as causing enhanced aging-dependent DA loss

- 52 crosses classified as 'high' (greater than $100 \%$ at 4 weeks age)

- 5 crosses resulted in lethality

$\circ$ Individual genes from lethal deficiencies crosses

- 1 gene resulting in lethality with ddc $>$ A53T identified as $k d n(C S)$

\subsection{Modification of $\alpha$-Synuclein[A53T] Toxicity by Mitochondrial Chaperone Protein, TRAP1}

\subsubsection{Modification of $\alpha$-Synuclein[A53T] Toxicity by TRAP1 in D. melanogaster}

\subsubsection{Effect of human TRAP1 overexpression on loss of brain DA in the}

fly

The finding that decreased TRAP1 expression could enhance $\alpha$-Synuclein[A53T] induced DA loss provoked the question of whether or not overexpression of TRAP1 could suppress $\alpha$-Synuclein[A53T] toxicity. To this end, a new transgenic fly expressing human TRAP1 (UAS-hTRAP1) was generated. Western blotting was used to assay whether or not induction of human TRAP1 expression or RNAi-mediated silencing of endogenous TRAP1 had effect on ectopic $\alpha$-Synuclein[A53T] expression. As is evident from Fig. IV-15, neither overexpression nor silencing of TRAP1 resulted in a change of $\alpha$-Synuclein abundance. Similarly, $\alpha$-Synuclein expression did not influence TRAP1 expression. The overexpression of human TRAP1 provided a rescue of brain DA loss in both ddc $>$ A53T and elav>A53T flies (Fig. IV-16). 

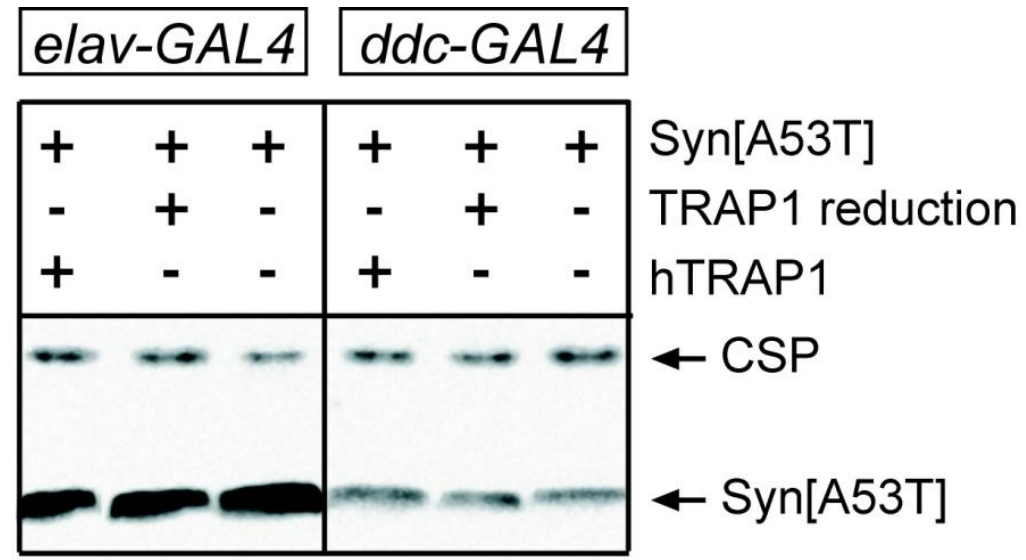

\section{elav-GAL4 ddc-GAL4}

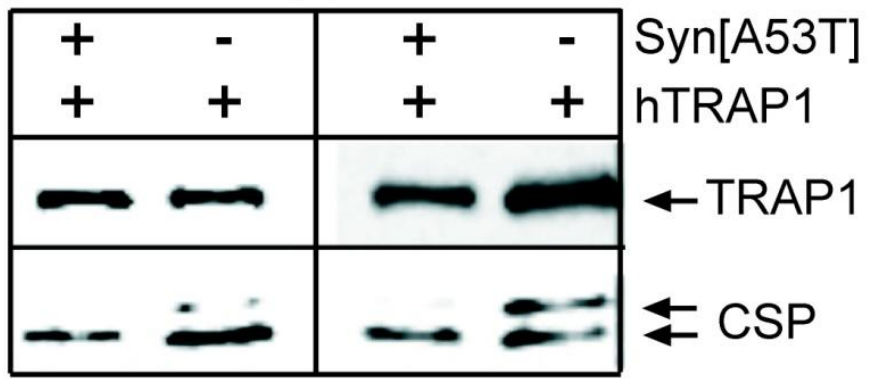

Fig. IV-15. D. melanogaster expression of human $\alpha$-Synuclein[A53T] and human TRAP1 under ddc and elav-GAL4 driver control. Protein of interest expression was detected on Western blots with specific antibodies for TRAP1 (75 kDa) and $\alpha$-Synuclein[A53T] (16 kDa) in reference to CSP- $\alpha$ (protein loading control, $34 \mathrm{kDa}$ ). A) Expression of Synuclein[A53T] expression. Top panel lanes from left to right: 1 . ddc $>$ A53T/+, 2. ddc >A53T/ TRAP1[KG06242], 3. ddc>A53T/hTRAP1, 4. elav>A53T/+ 5. elav>A53T/ TRAP1-

RNAi[3152R1], 6. elav>A53T/hTRAP1. B) Expression of TRAP1. Bottom panel lanes from left to right: 1. ddcGAL/hTRAP1, 2. ddc >A53T/hTRAP1 , 3. elav-GAL/hTRAP1, 4. elav>A53T/hTRAP1. CSP- $\alpha$ antibody shows binding to isoform 1 and 2 in TRAP1 blot (34, $36 \mathrm{kDa})$. Representative blots after 3 experiments shown.

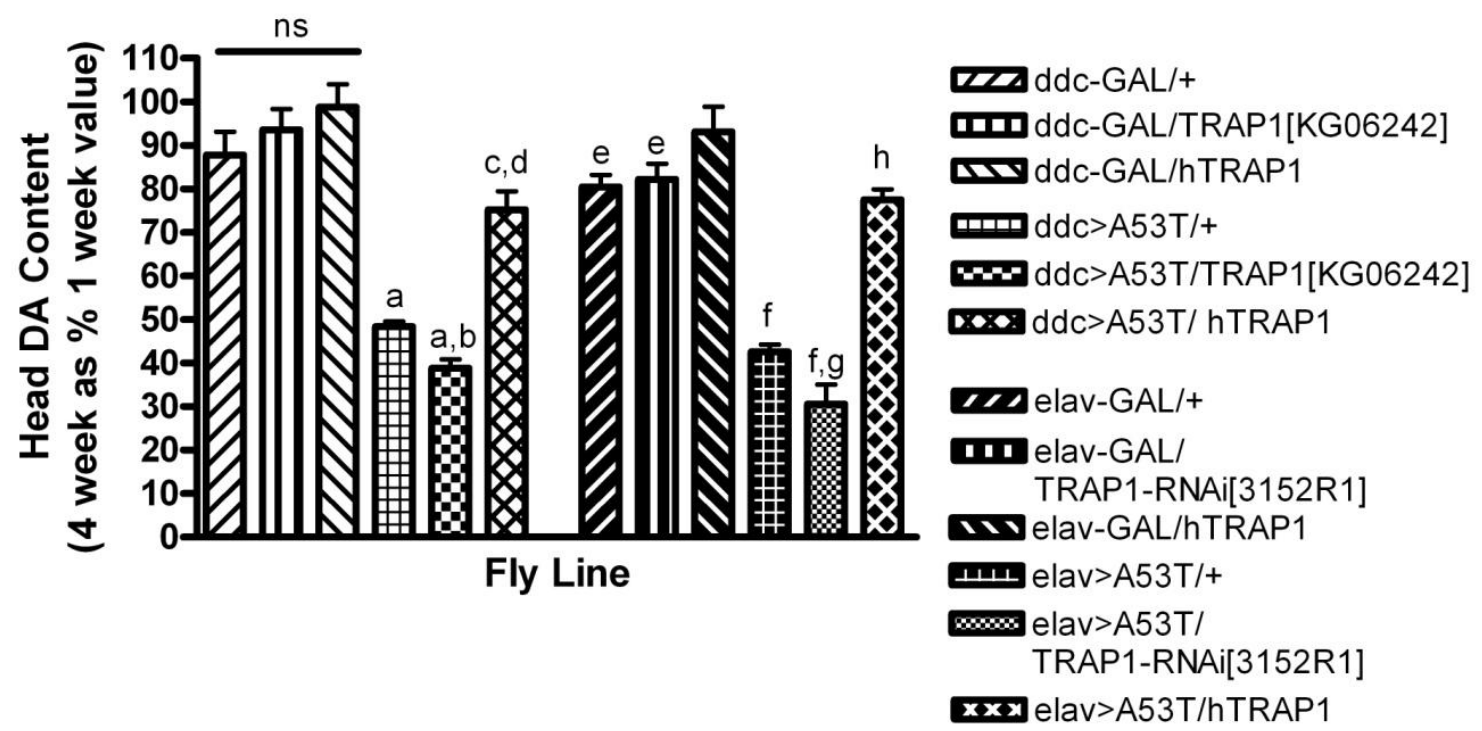

Fig. IV-16. Aging-dependent loss of brain DA in flies expressing a-Synuclein[A53T] with hTRAP1 expression or reduced endogenous TRAP1 expression (ddc-GAL4 or elav-GAL4 driver). Flies were aged and collected at 1 and 4 weeks for measurement of head DA content using HPLC. Significant differences 
(separate 1-way ANOVA for each driver-line set) observed: a: $\mathrm{p}<0.001$ vs. ddc-GAL/+; b: $\mathrm{p}<0.01$ vs. ddc $>$ A53T/+; c: $p<0.05$ vs. ddc-GAL/+; d: p<0.001 vs. ddc $>$ A53T/+; e: $p<0.05$ vs. elav-GAL/hTRAP1; f: $\mathrm{p}<0.001$ vs. elav-GAL/+; g: $\mathrm{p}<0.05$ vs. elav $>A 53 \mathrm{~T} /+$; $\mathrm{h}$ p $<0.001$ vs. elav $>A 53 \mathrm{~T} /+(\mathrm{n}=10$ for ddc and elav $>\alpha-$ Synuclein[A53T] flies with/out TRAP1 reduction; otherwise $n=6$ ).

\subsubsection{Sensitivity to oxidative stress in flies expressing $\alpha$-Synuclein[A53T]} and alteration to TRAP1 expression

Altered sensitivity to oxidative stress has been noted for a number of PD-related genes. Chronic treatment of D. melanogaster with either the pesticide rotenone, a mitochondrial complex I inhibitor, or the herbicide paraquat, a powerful oxidant, which can interfere with mitochondrial function resulting in Caspase -3 cell death, has been shown to result in neurodegenerative and behavioral alteration that recapitulates key features of PD. ${ }^{222 \text {, }}$ ${ }^{225,} 226$ In addition, oxidative stress treatment in the context of $\alpha$-Synuclein overexpression has been shown to increase $\alpha$-Synuclein aggregation and inclusion formation, with an enhancement of toxicity. $^{227,228}$ At the same time, cell culture experiments have shown TRAP1 to be a protective factor against oxidative stress- induced damage and apoptosis. ${ }^{229,230}$ Thus, to evaluate whether TRAP1 altered fly sensitivity in the context $\alpha$-Synuclein[A53T] expression, newly hatched flies were treated with either paraquat or hydrogen peroxide (toxic due to reaction with ferrous iron to produce the $\bullet \mathrm{OH}$ radical, resulting in free radical damage to cellular contents, cytosolic acidification and possible inhibition of glycolysis). ${ }^{231}$

Flies expressing $\alpha$-Synuclein[A53T] under the ddc-GAL4 driver showed an enhanced sensitivity to paraquat treatment (Fig. IV-17). Reduction of TRAP1 expression in these flies further enhanced paraquat sensitivity. However, overexpression of TRAP1, when in combination with $\alpha$-Synuclein[A53T], enhanced survival. Similarly, the combination of $\alpha-$ Synuclein[A53T] expression and TRAP1 reduction enhanced sensitivity to hydrogen peroxide treatment, while overexpression of TRAP1 with $\alpha$-Synuclein[A53T] enhanced survival. $\alpha$ Synuclein[A53T] expression alone, however, did not reduce survival upon hydrogen peroxide treatment (Fig. IV-18). A decrease in TRAP1 expression was also sufficient to reduce survival upon hydrogen peroxide treatment. This is the first known data in an animal model indicating 
a decrease in TRAP1 can detrimentally affect response to oxidative stress, which confirms previous data from cell culture systems. Curiously, the least sensitive flies to hydrogen peroxide treatment were ddc $>$ A53T/hTRAP1 and not ddc-GAL/hTRAP1. The same experiments for oxidative stress (hydrogen peroxide, paraquat) were also repeated using the elav-GAL4 driver and results were similar to those obtained using ddc-GAL4 driver.

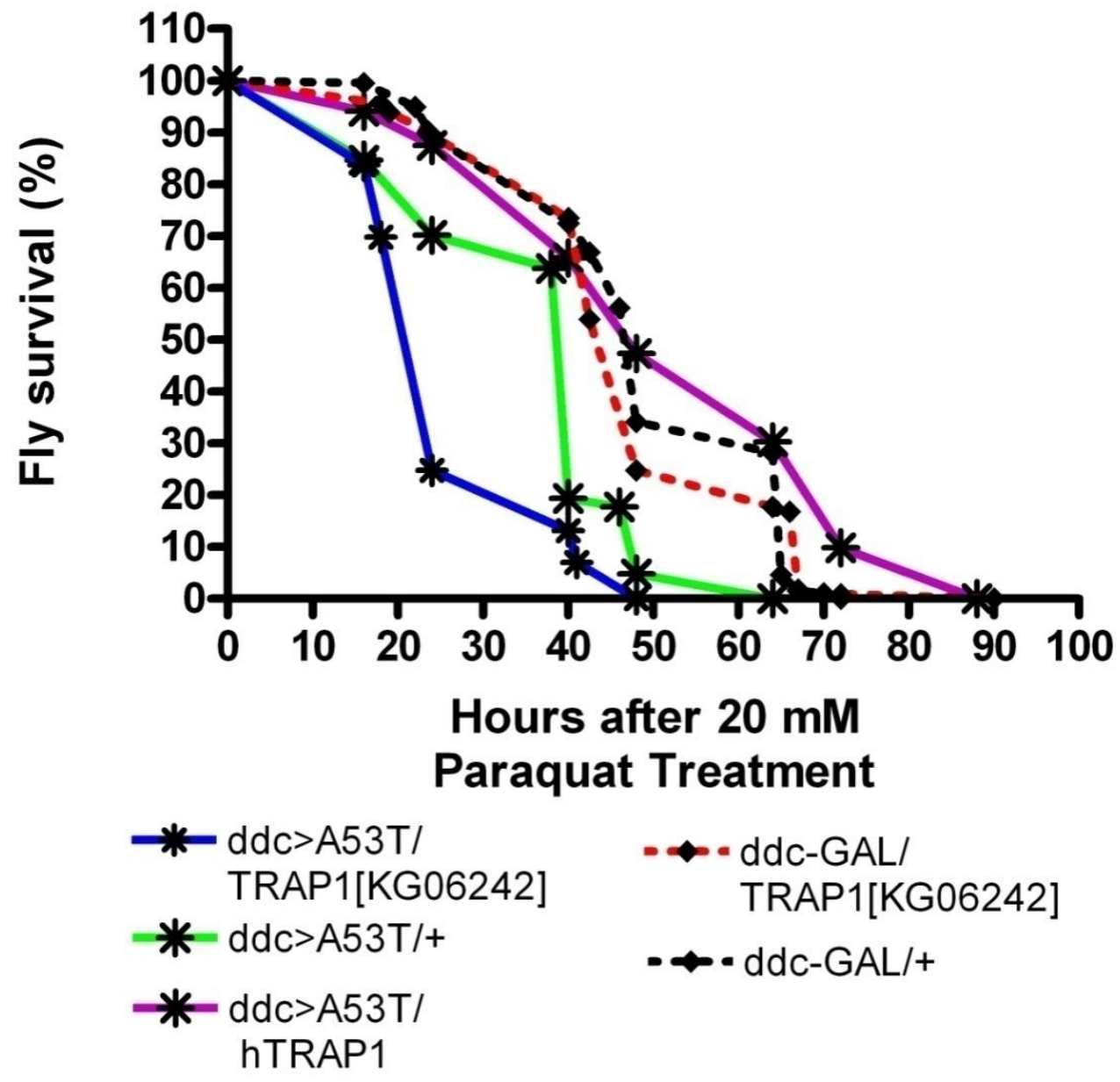

Fig. IV-17. Fly survival after paraquat $(20 \mathrm{mM})$ treatment ( $\alpha$-Synuclein[A53T] expression with hTRAP1 expression or reduced endogenous TRAP1 expression (ddc-GAL4 driver)). Flies expressing $\alpha$ Synuclein[A53T] show reduced survival after treatment in comparison to control flies $(\mathrm{p}<0.0001$, ddc-GAL/+ vs. ddc $>$ A53T/+). Flies with reduced TRAP1 expression and $\alpha$-Synuclein[A53T] show enhanced sensitivity beyond $\alpha$-Synuclein[A53T] alone $(\mathrm{p}<0.0001, \mathrm{ddc}>\mathrm{A} 53 \mathrm{~T} /+\mathrm{vs.} \mathrm{ddc}>\mathrm{A} 53 \mathrm{~T} / \mathrm{TRAP} 1[\mathrm{KG06242]})$. Overexpression of human TRAP1 with $\alpha$-Synuclein[A53T] reduces paraquat sensitivity $(p<0.0001$, ddc $>$ A53T/ + vs. ddc $>$ A53T/hTRAP1). Reduction of TRAP1 expression alone also reduces survival after paraquat treatment $(\mathrm{p}=0.01$, ddc-GAL/+ vs. ddc-GAL/TRAP1[KG06242]). Although the curves are significantly different ( $\mathrm{p}<0.001$ ), survival time is not different for $\mathrm{ddc}>\mathrm{A} 53 \mathrm{~T} / \mathrm{hTRAP} 1$ and ddc-GAL/+, indicating a restoration to control with TRAP1 overexpression. Human TRAP1 expression alone did not significantly change sensitivity to paraquat ( $\mathrm{p}=\mathrm{ns}$, ddc-GAL/+ vs. ddc-GAL/hTRAP1, survival curve not shown). $\mathrm{n}>100$. 


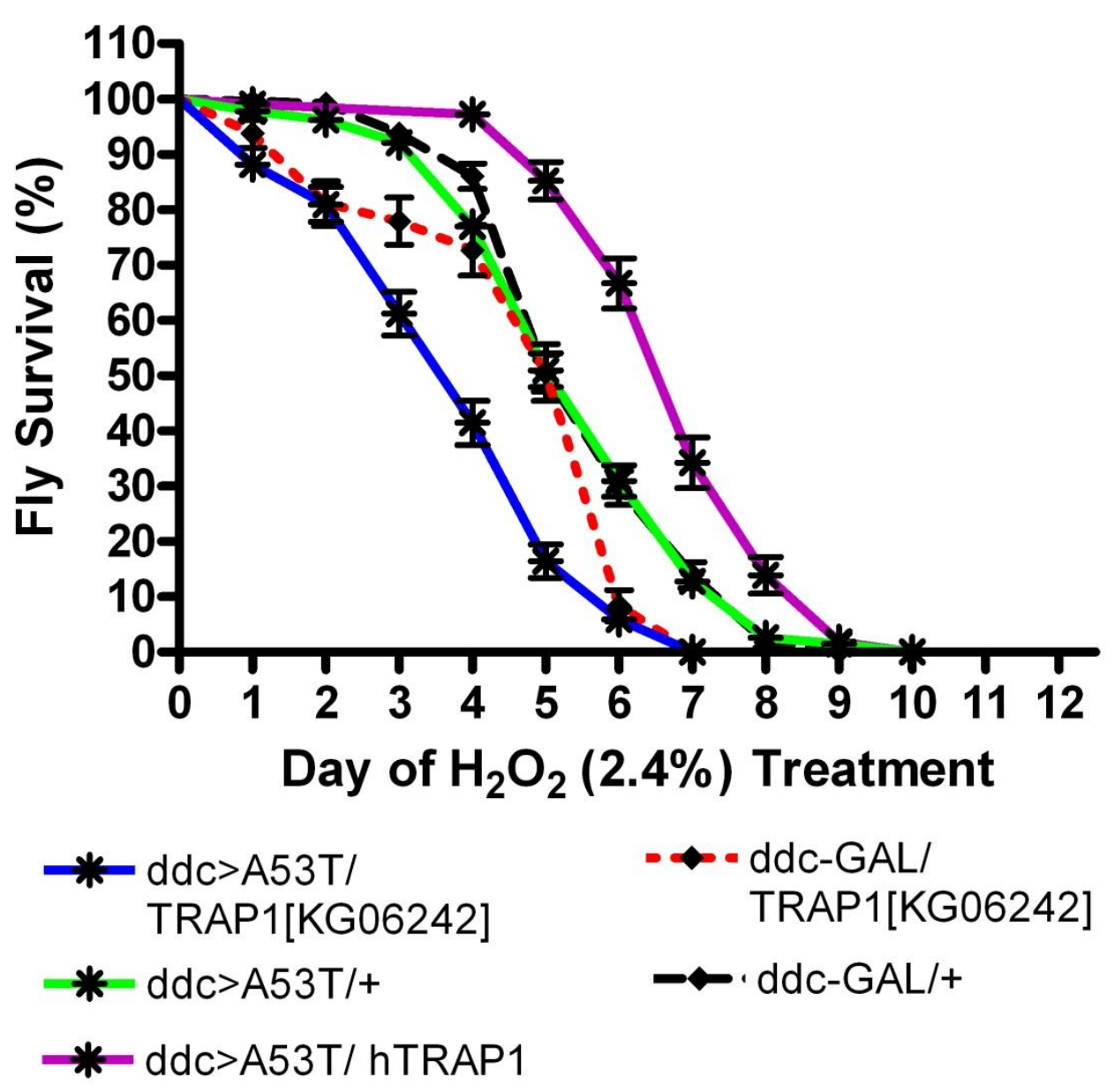

Fig. IV-18. Fly survival after hydrogen peroxide (2.4\%) treatment ( $\alpha$-Synuclein[A53T] expression with hTRAP1 expression or reduced endogenous TRAP1 expression (ddc-GAL4 driver)). Flies with reduced TRAP1 expression and $\alpha$-Synuclein[A53T] show enhanced sensitivity $(\mathrm{p}<0.0001$, ddc $>$ A53T/+ vs. ddc $>$ A53T/ TRAP1[KG06242]). $\alpha$-Synuclein[A53T] expression alone did not enhance sensitivity to hydrogen peroxide ( $\mathrm{p}=\mathrm{ns}$, ddc-GAL/+ vs. ddc $>$ A53T/+). Overexpression of human TRAP1 with $\alpha$-Synuclein[A53T] reduces hydrogen peroxide sensitivity ( $\mathrm{p}<0.0001$, ddc $>$ A53T/+ vs. ddc $>$ A53T/ hTRAP1), however hTRAP1 expression alone was not different than control ( $\mathrm{p}=\mathrm{ns}$, ddc-GAL/+ vs. ddc-GAL/hTRAP1, survival curve not shown). Flies with reduced TRAP1 expression show altered survival time course vs. control ( $\mathrm{p}<0.001$, ddc-GAL/+ vs. ddcGAL/ TRAP1[KG06242]) (n>100).

\subsubsection{Negative geotaxis assay of flies expressing $\alpha$-Synuclein[A53T] with alteration to TRAP1 expression}

PD is clinically defined as a movement disorder and thus key to animal model disease phenotype recapitulation is loss of locomotor ability. In flies, the negative geotaxis assay or a climbing assay has been used to assess fly locomotor ability in both PD and aging models. After tapping flies to the bottom of a vial, normal flies will quickly climb to the top of the vial. Previous reports indicate that flies expressing $\alpha$-Synuclein show an age-related deficit in 
climbing ability. ${ }^{135,209}$ Of interest was thus whether or not overexpression of human TRAP1 could rescue locomotor defects.

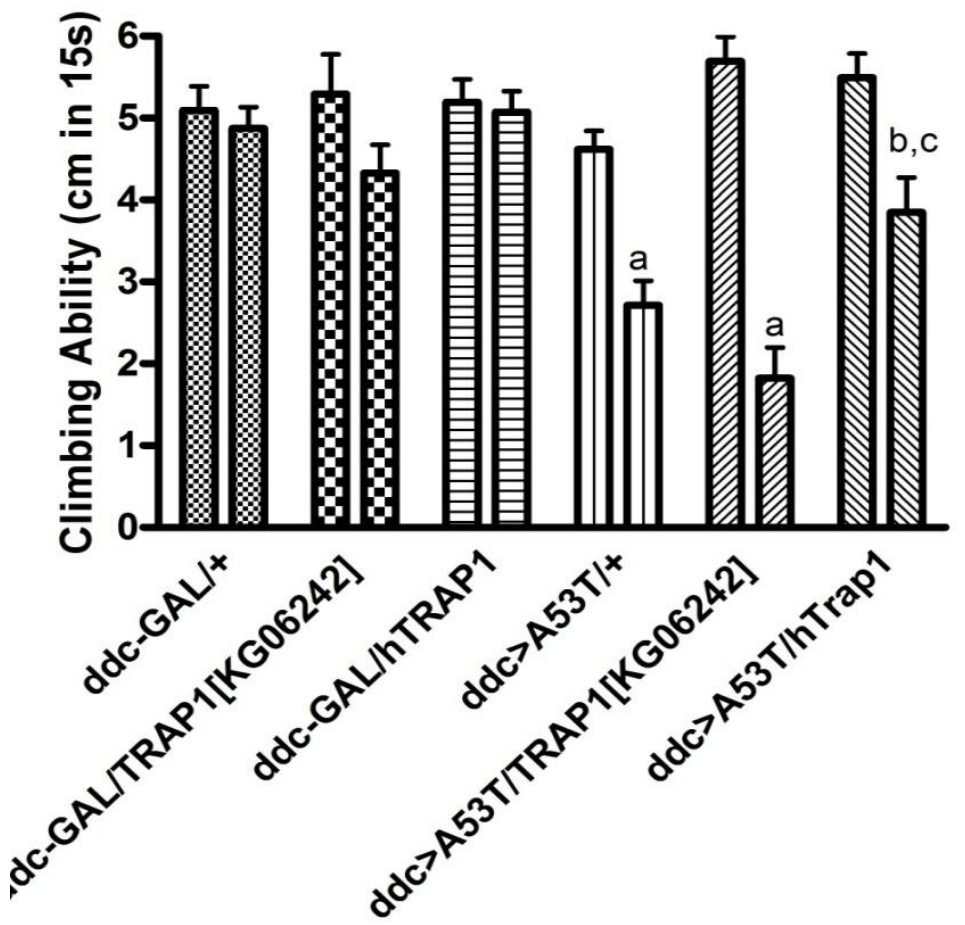

Fly Age (1 and 4 weeks)

Fig. IV-19. Fly climbing ability with age ( $\alpha$-Synuclein[A53T] expression with hTRAP1 expression or reduced endogenous TRAP1 expression (ddc-GAL4 driver)). Climbing ability was assessed at ages 1 and 4 weeks. Significant differences (2-way ANOVA) observed: a: $p<0.001$ vs. 4 week ddc-GAL/+; b: $p<0.05$ vs. 4 week ddc $>$ A53T]/+; c: p $<0.001$ vs. 4 week ddc $>$ A53T/ TRAP1[KG06242] $(n=20)$.

Flies were tested at ages 1 and 4 weeks for climbing ability, the same time points as for HPLC DA measurements. Confirming previous reports, flies expressing $\alpha$ Synuclein[A53T] showed a decrease in climbing ability that is age-dependent (measured using screening specific flies, that is, ddc-GAL4 driver, Fig. IV-19) ${ }^{135}$ At 4 weeks of age, ddcGAL4 driver flies showed an age-dependent locomotor defect specific to $\alpha$-Synuclein[A53T], as control flies (driver alone, TRAP1 reduction using P-element disruption allele TRAP1[KG06242] and over expression of hTRAP1), did not show significant loss of locomotor ability. Importantly, overexpression of human TRAP1 was able to rescue the loss of locomotor ability resulting from $\alpha$-Synuclein[A53T] expression (4 week data ddc $>$ A53T/hTRAP1 not different than 4 week ddc-GAL/+ data). Experiment repeated using 
the elav-GAL4 showed similar results to ddc-GAL4. Thus, this data indicates that rescue of brain DA content was sufficient to restore motor ability.

\subsubsection{Loss of DA neurons in flies expressing $\alpha$-Synuclein[A53T] and alteration to TRAP1 expression}

Previously reported Drosophila modifiers of $\alpha$-Synuclein such as HSP70 have been shown to be able to restore DA neuron numbers to control levels. ${ }^{142}$ While the present data set has already shown that TRAP1 overexpression can increase brain DA and motor ability of aged $\alpha$-Synuclein[A53T] flies, this restoration could in principle be effected by increased health of remaining DA neurons, without rescue of cells per se. Thus, key dopaminergic clusters in flies with TRAP1 knockdown or human TRAP1 overexpression were compared for numbers of TH-positive neurons at 4 weeks of age (Fig. IV-20). In both the PPM1/2 and PPL1 clusters, TRAP1 knockdown result in an significant loss of neurons beyond that seen with $\alpha$-Synuclein[A53T] expression alone. Decreased TRAP1, in the absence of $\alpha$ Synuclein[A53T], did not affect DA neuronal numbers.

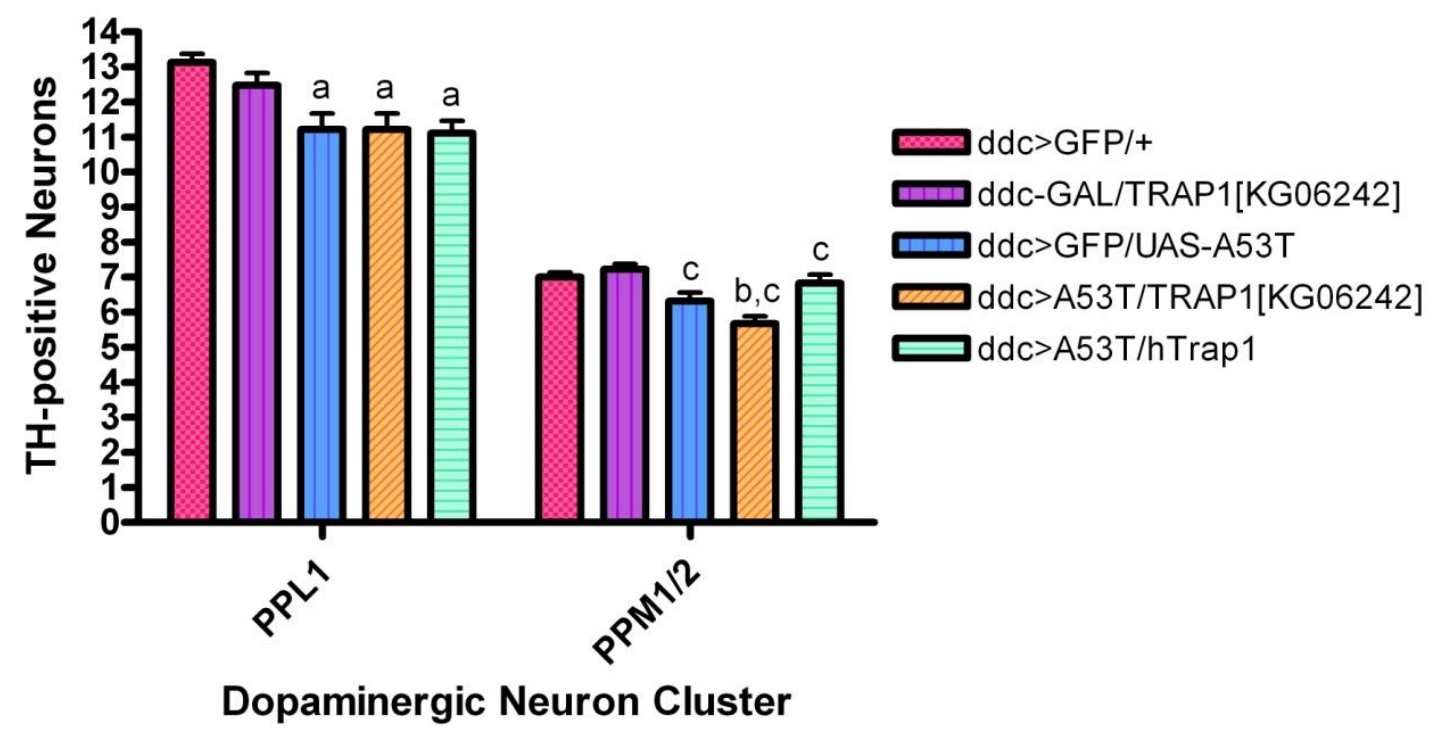

Fig. IV-20. Fly brain dopaminergic neuron numbers at 4 weeks of age. Brains from aged flies were dissected, fixed and stained before DA neuron cluster imaging under the confocal microscope. PPL1 and PPM1/2 neuron cluster counts from flies expressing $\alpha$-Synuclein[A53T] were compared to flies with $\alpha$-Synuclein[A53T] and either a TRAP1 reduction allele (TRAP1[KG06242]) or human TRAP1. Control flies (ddc $>$ GFP/+) were also compared to flies with the TRAP1 reduction allele. Significant differences(1-way ANOVA for each cluster) 
observed: a: $p<0.05$ vs. ddc $>$ GFP/lacZ; b: $p<0.001$ vs. ddc $>$ GFP/+; : $p<0.05$ vs. ddc $>$ GFP/UAS-A53T. Brains $\mathrm{n}>15$.

\subsubsection{Longevity of flies expressing $\alpha$-Synuclein[A53T] with alteration to}

\section{TRAP1 expression}

Fly longevity with TRAP1 expression alteration was also investigated for flies associated with screening. Under the ddc-GAL4 driver with $\alpha$-Synuclein[A53T] expression, there were no significant differences in longevity (see Appendix Results Fig. A2-1).

\subsubsection{Modification of $\alpha$-Synuclein[A53T] Toxicity by TRAP1 in Human HEK293 Cell Culture}

\subsubsection{Knockdown and overexpression of TRAP1 in $\alpha$-Synuclein[A53T]- expressing cells}

In order to confirm the results generated in the fly concerning TRAP1-rescue of $\alpha$ Synuclein[A53T]-induced toxicity in a vertebrate system, human and rat cell culture was used. In addition, the use of human or rat cell culture offers several advantages including more extensive antibody collection vs. that available for Drosophila, direct biochemical assay of mitochondrial- related factors, protein-protein interactions, and initial screening of new mutants. For a more complete introduction into cell culture model, please see Introduction section 2.3.1..

siRNA was used to knockdown TRAP1 expression in HEK293 cells. Validation of the delivery system and concentration required for gene knockdown was first confirmed using siRNA for MAPK1 (data in Appendix Methods Figs. A, B). Two different siRNAs directed against TRAP1 were compared for efficacy. As shown in Fig. IV-22, both TRAP1-siRNAs were able to reduce endogenous TRAP1-expression in HEK cells. However, siTRAP1-1 was shown to be more efficient and was therefore used for further investigations. 


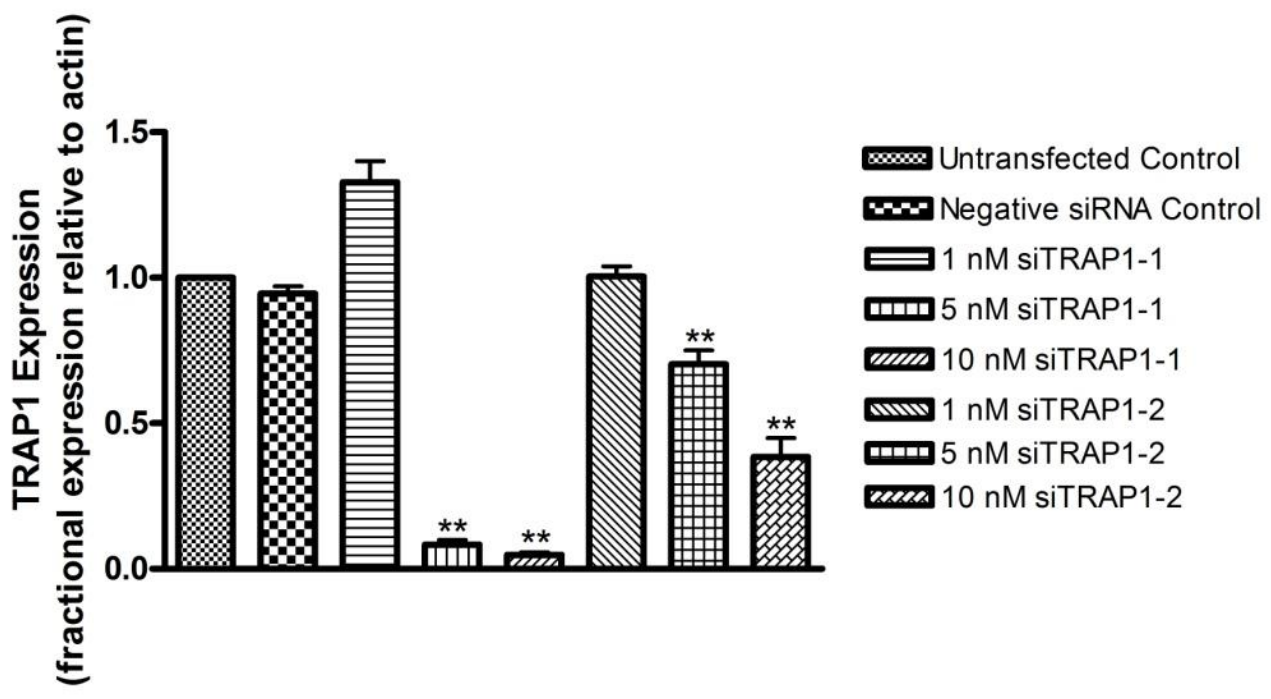

Fig. IV-22. Comparison of siRNA efficacy for knockdown of TRAP1 in HEK293 cells. Assessment of relative TRAP1 expression levels. Relative abundance of endogenous TRAP1 transcripts in relation to $\beta$-actin. Both siTRAP1-1 and siTRAP1-2 resulted in knockdown of TRAP1 expression (**, $\mathrm{p}<0.01$ vs. Negative siRNA control). $n=3$.

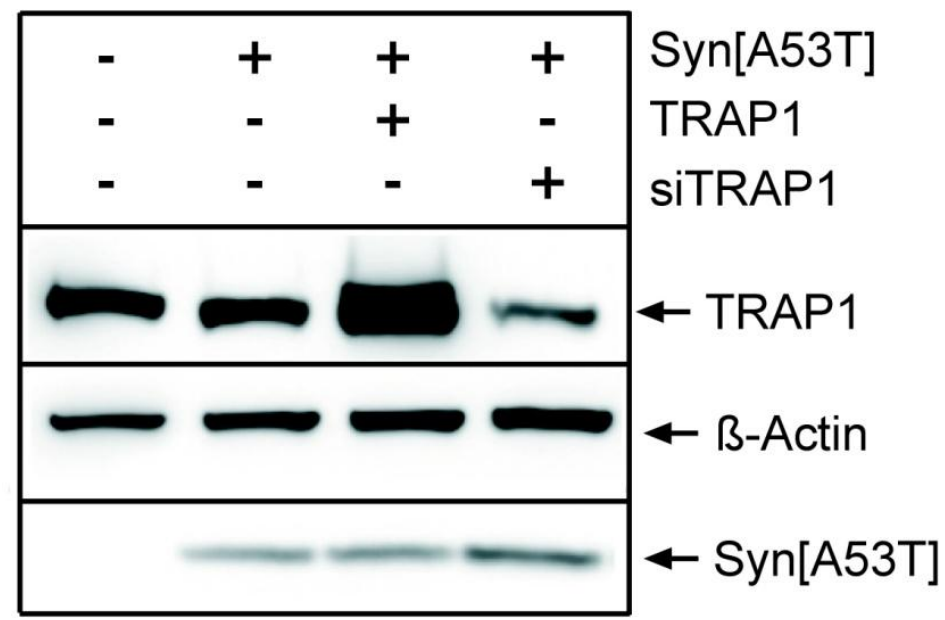

Fig. IV-23. Protein expression of $\alpha$-Synuclein[A53T], TRAP1 and siTRAP1 in HEK293 cell culture. Western blot analysis of HEK cell lysates probed with TRAP1 (75 kDa), $\beta$-actin (50 kDa) and $\alpha$-Synuclein (16 $\mathrm{kDa})$ specific antibodies. HEK cells were either (lanes from left to right) untransfected (control), or transfected with $\alpha$-Synuclein[A53T] (Control vector+ $\alpha$-Synuclein[A53T]), co-transfected with $\alpha$-Synuclein[A53T] and TRAP1 ( $\alpha$-Synuclein[A53T]+ TRAP1), or siTRAP1 ( $\alpha$-Synuclein[A53T] + siTRAP1). Blot representative of 3 experiments.

Next the effect of TRAP1-silencingand overexpression was analyzed on a protein level (Fig.

IV-23). TRAP1-overexpression as well as TRAP1 silencing resulted in enhanced/decreased protein levels, respectively. In addition, overexpression or silencing of TRAP1 was tested for possible effect on co-expressed $\alpha$-Synuclein[A53T] levels. This was not the case (data not shown). 
Previous reports showed a localization of TRAP1 to mitochondria. Co-localization studies in HEK cells revealed a strong overlap of TRAP1 staining with "Mitotracker Orange"labelled mitochondria. This indicates that TRAP1 indeed localizes to mitochondria in HEK cells (Fig. IV-24).

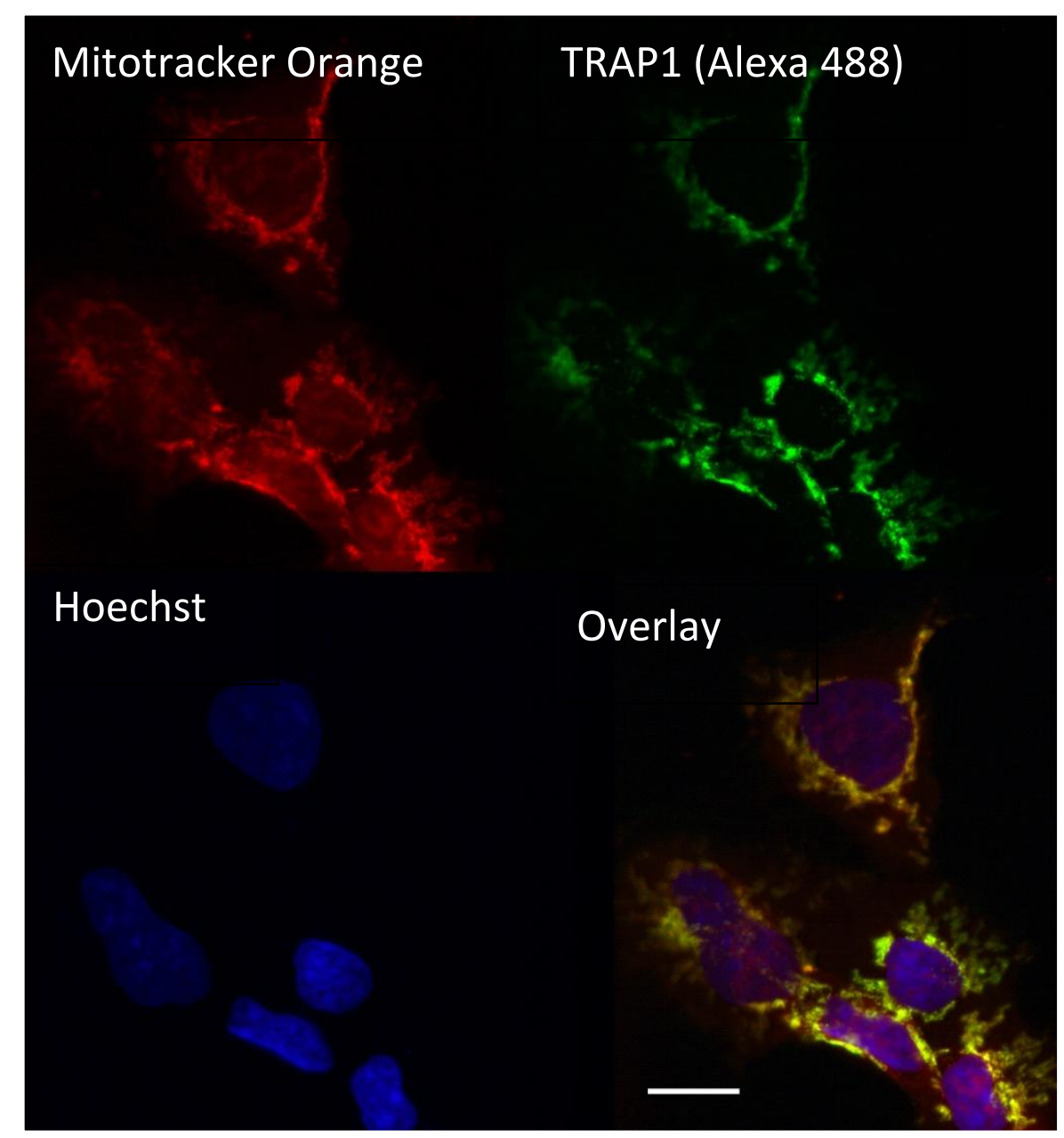

Fig. IV-24. Localization of TRAP1 to mitochondria. HEK293 cells were stained using the mitochondrial marker "Mitotracker Orange" (red), TRAP1 (green) and Hoechst nuclear stain (blue). A high degree of colocalization of red and green fluorescent signals is apparent in overlay. Scale bar indicates $27 \mu \mathrm{m}$. Slides imaged on Leica fluorescent microscope.

Immunocytochemical analysis on HEK cells using a TRAP1 specific antibody indicated that these cells have a robust endogenous expression of TRAP1 as evident by strong mitochondrial staining. This staining was highly reduced if cells were treated with siTRAP1 and strongly increased in cells with TRAP1 overexpression. Also in this analysis, $\alpha-$ Synuclein[A53T] protein levels did not seem to change upon TRAP1 overexpression/silencing (Fig. IV-25). 


\section{RESULTS}

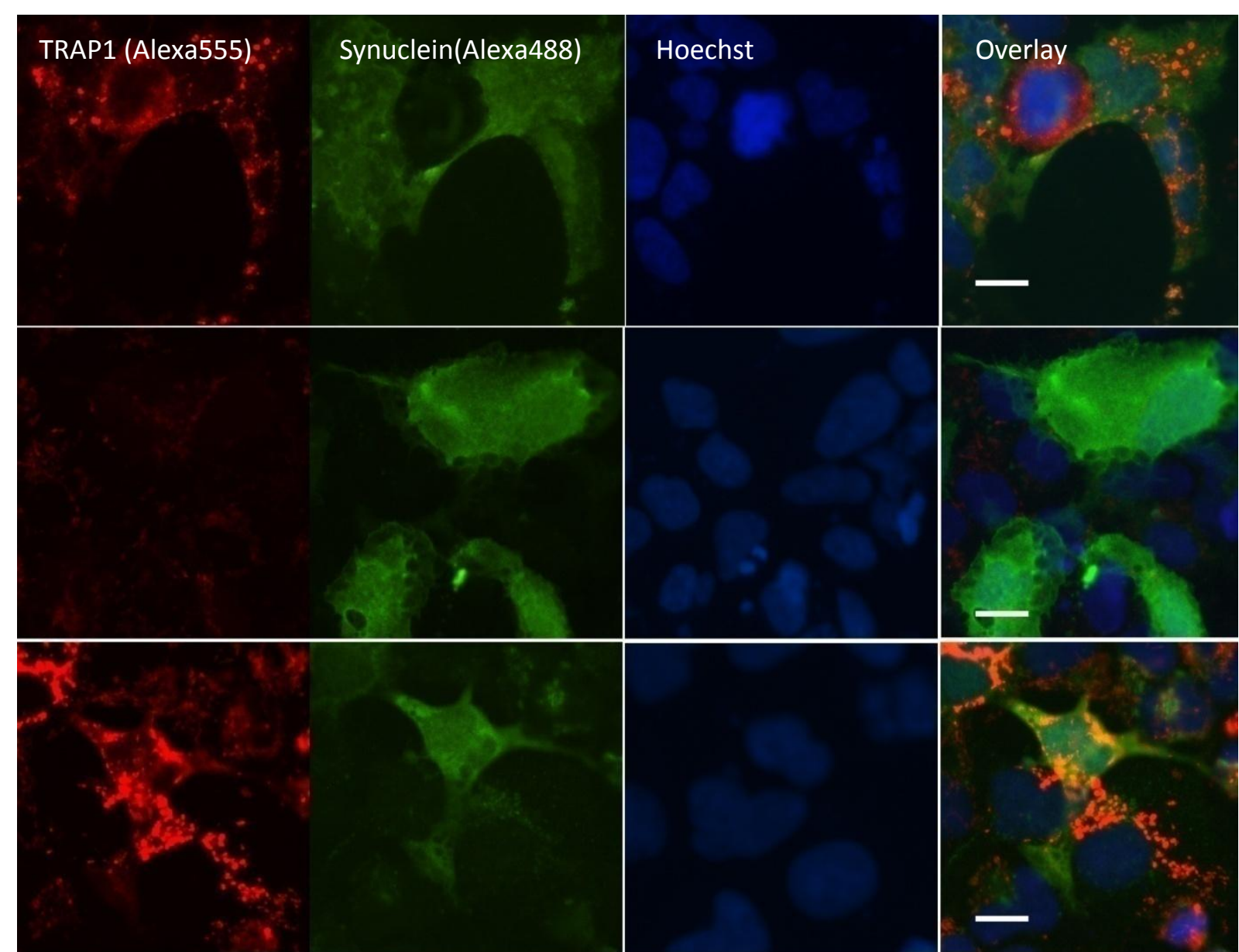

Fig. IV-25. TRAP1 overexpression and downregulation in HEK293 with $\alpha$-Synuclein[A53T] expression. HEK cells transfected with $\alpha$-Synuclein[A53T] were stained for TRAP1 (red), $\alpha$-Synuclein[A53T] (green) and DNA (blue). Merged picture is shown (overlay). Upper panel: $\alpha$-Synuclein[A53T] expressing cells cotransfected with empty vector. Middle panel: $\alpha$-Synuclein[A53T] expressing cells with siTRAP1. Lower panel: Cells with $\alpha$-Synuclein[A53T] and TRAP1 overexpression. Scale bar $=24 \mu \mathrm{m}$.

\subsubsection{2. $\alpha$-Synuclein[A53T] mitochondrial localization using immunofluorescence and confocal microscopy}

TRAP1 is defined as a mitochondrial molecular chaperone and is protective against cell death and in particular, oxidative stress- induced cell death via multiple postulated mechanisms, including inhibition of Cytochrome c release, interference with Caspase 3

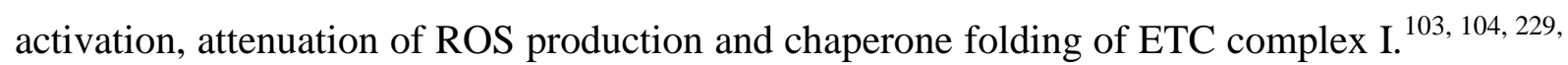
230, 232-234 At the same time, multiple recent papers have suggested that $\alpha$-Synuclein's toxic effect may in part result from direct effects on the mitochondria and that a cryptic mitochondrial targeting allows its entry. ${ }^{46,129,174,176,235}$ TRAP1, as a mitochondrial protein, is able to rescue toxicity induced $\alpha$-Synuclein. This leads to the hypothesis, that $\alpha$-Synuclein 
toxicity is at least partially linked to mitochondrial function and that these effects can be directly antagonized by TRAP1. Of interest was therefore to confirm, as previously reported, that $\alpha$-Synuclein can indeed be found within the mitochondria. ${ }^{46,174,177}$ Two methods were used to show that $\alpha$-Synuclein at least partially localizes to mitochondria: 1 . Co-localization of $\alpha$-Synuclein with mitochondria using immunofluorescence and 2. cell fractionation assays with subsequent Western blot analysis to address $\alpha$-Synuclein abundance in the mitochondrial fraction.

Previously published data suggested that $\alpha$-Synuclein mitochondrial entry was stimulated by low $\mathrm{pH}$ and oxidative stress. ${ }^{180}$ Thus, after stressing $\alpha$-Synuclein[A53T]expressing HEK293 cells with rotenone, cells were examined for evident co-localization of $\alpha$ Synuclein[A53T] and mitochondria. While a large proportion of $\alpha$-Synuclein[A53T] was found in the cytoplasm, a small proportion of $\alpha$-Synuclein[A53T] seemed also to be located within the mitochondria (Fig. IV-26).

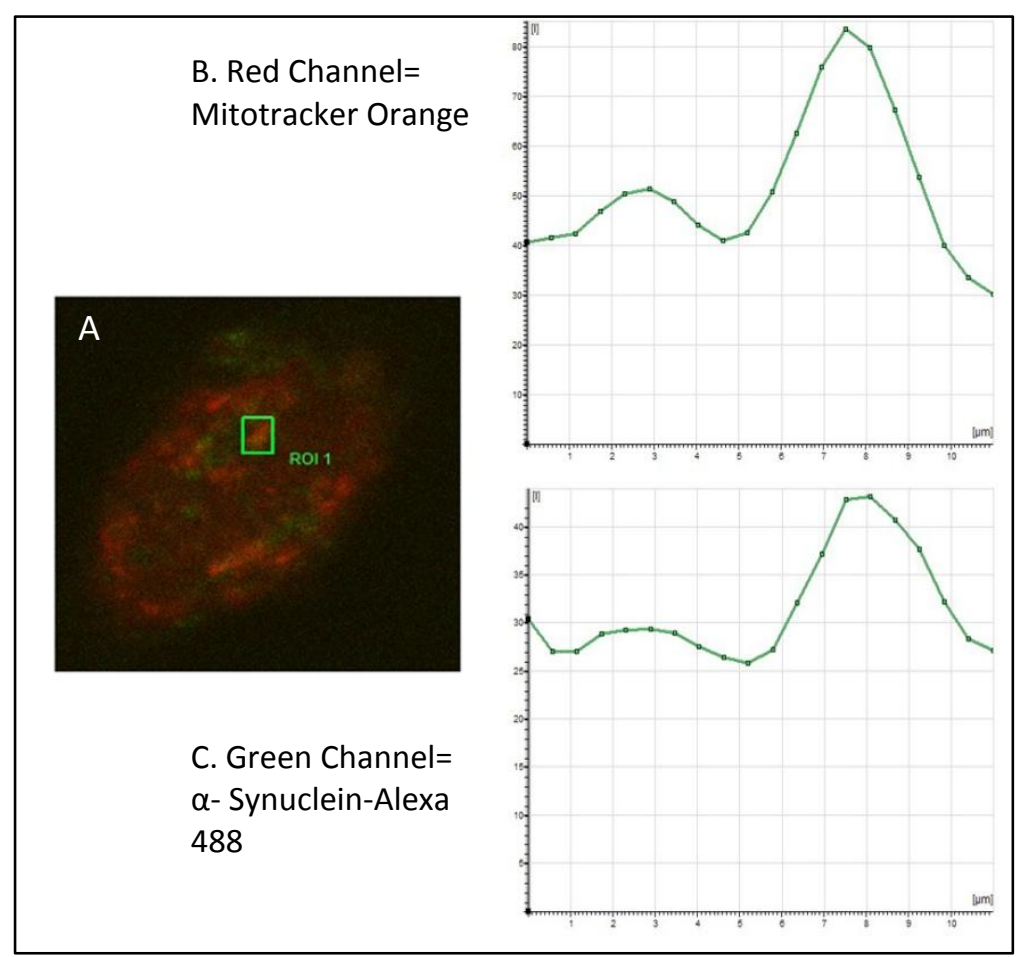

Fig. IV-26. Co-localization of fluorescent signal from Mitotracker Orange and $\alpha$-Synuclein[A53T]Alexa488. HEK cells were stained with Mitotracker Orange (red) and $\alpha$-Synuclein[A53T] specific antibody (green).Confocal Z-stacks were analyzed for localization of fluorescent signal intensity in indicated region of interest $\left(3.42 \mu \mathrm{m}^{2}\right)$. Fluorescence distribution of red (upper) and green (lower) signals across the ROI is shown. 


\subsubsection{3. $\alpha$-Synuclein[A53T] mitochondrial localization using cell fractionation}

In addition to immunofluorescence assays for $\alpha$-Synuclein[A53T] localization, cell fractionation was used to further the argument that $\alpha$-Synuclein[A53T] can be found within the mitochondria. The process of mitochondrial isolation divided cellular contents into three fractions: a mitochondrial enriched fraction, a light cytoplasmic fraction and a crude pellet. Using Western blotting, these fractions were compared for protein content of TRAP1 (mitochondrial protein, putatively localized to the matrix), VDAC1 (mitochondrial outer membrane protein), Cytochrome $\mathrm{c}$ (inner mitochondrial membrane). All three proteins showed enrichment in the mitochondrial fraction, with Cytochrome c banding noted only within the mitochondrial fraction, indicating specificity of the mitochondrial enrichment procedure (Fig. IV-27). Importantly, $\alpha$-Synuclein[A53T] protein band was also found within the mitochondrial fraction. Thus, the combined data of both immunofluorescence and

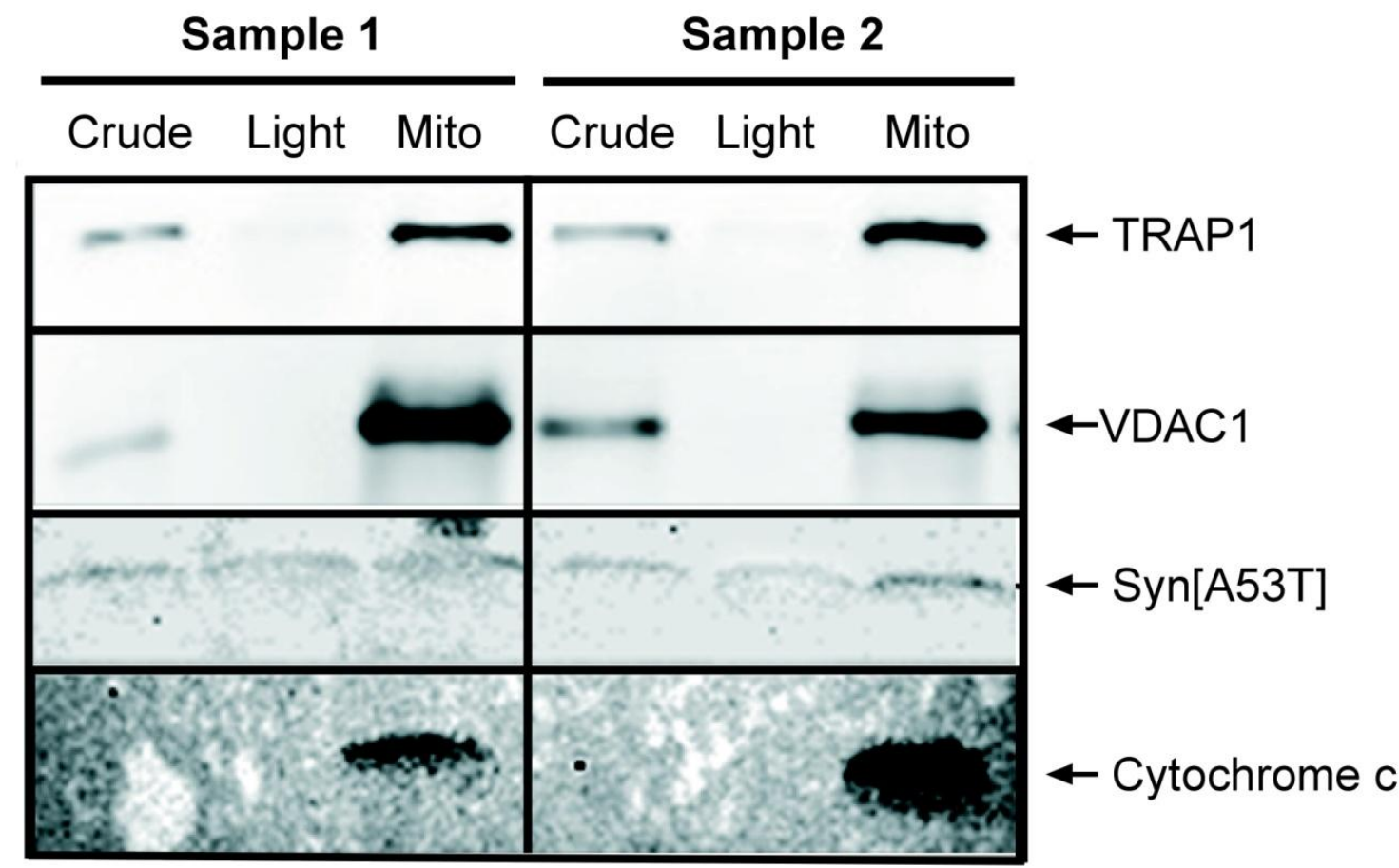

Fig. IV-27. Mitochondrial cell fraction isolation from HEK293 cells expressing $\alpha-S y n u c l e i n[A 53 T]$. Samples derived from two independent cell fractionation assays were used for Western blot analysis. Blots were probed with specific antibodies detecting the following proteins: TRAP1 (75 kDa), VDAC1 (31 kDa), $\alpha-$ Synuclein $(16 \mathrm{kDa})$ and Cytochrome $\mathrm{c}(15 \mathrm{kDa})$. Fractions analyzed (crude cell pellet; light cytoplasmic and 
mitochondrial enriched fraction) are indicated. Molecular weight of analyzed proteins is indicated. Representative blots from 3 experiments.

mitochondrial fractionation are suggestive that a small portion of $\alpha$-Synuclein[A53T] does indeed enter the mitochondria.

\subsubsection{Oxidative stress sensitivity of HEK293 cells expressing $\alpha$ - Synuclein[A53T], TRAP1 and/or siTRAP1}

Overexpression of TRAP1 has previously been shown to be protective against oxidative stress induced cell death via reduction in ROS production, restoration of mitochondrial membrane potential and inhibition of Cytochrome c release. ${ }^{105,229,236,237}$ At the same time, toxicity associated with $\alpha$-Synuclein[A53T] is in part mediated due to above mentioned mechanisms and moreover has been shown to sensitize cells to oxidative stress. ${ }^{238}$ Thus, to firstly determine whether in vitro treatment of cells mimicked the in vivo Drosophila data concerning TRAP1 and oxidative stress sensitivity, HEK293 cells were treated overnight with either hydrogen peroxide or rotenone. Rotenone is a specific Complex I inhibitor, while hydrogen peroxide is a more general oxidative stress inducer, mainly by producing primarily hydroxyl radicals. In both cases, $\alpha$-Synuclein[A53T] expression served to significantly enhance cell sensitivity to oxidative stressors (Figs. IV-28, 29). Reduction in TRAP1 expression with siRNA further reduced survival in presence of $\alpha$-Synuclein[A53T]. Moreover, reduction of TRAP1 was sufficient to decrease cell survival under oxidative stress in the absence of $\alpha$-Synuclein[A53T] overexpression. For both rotenone and hydrogen peroxide treatment, overexpression of TRAP1 in the context of $\alpha$-Synuclein[A53T] expression attenuated the decrease in cell survival. The magnitude of the rescue effect was greatest when cells were exposed to rotenone oxidative stress. Also in cells without $\alpha$ Synuclein[A53T] expression, the decrease in cell survival under oxidative stress was rescued to control level, independent of the used stressor. This data is in line with the reported function of TRAP1 as a protective mitochondrial chaperon. ${ }^{106,229,230}$ 


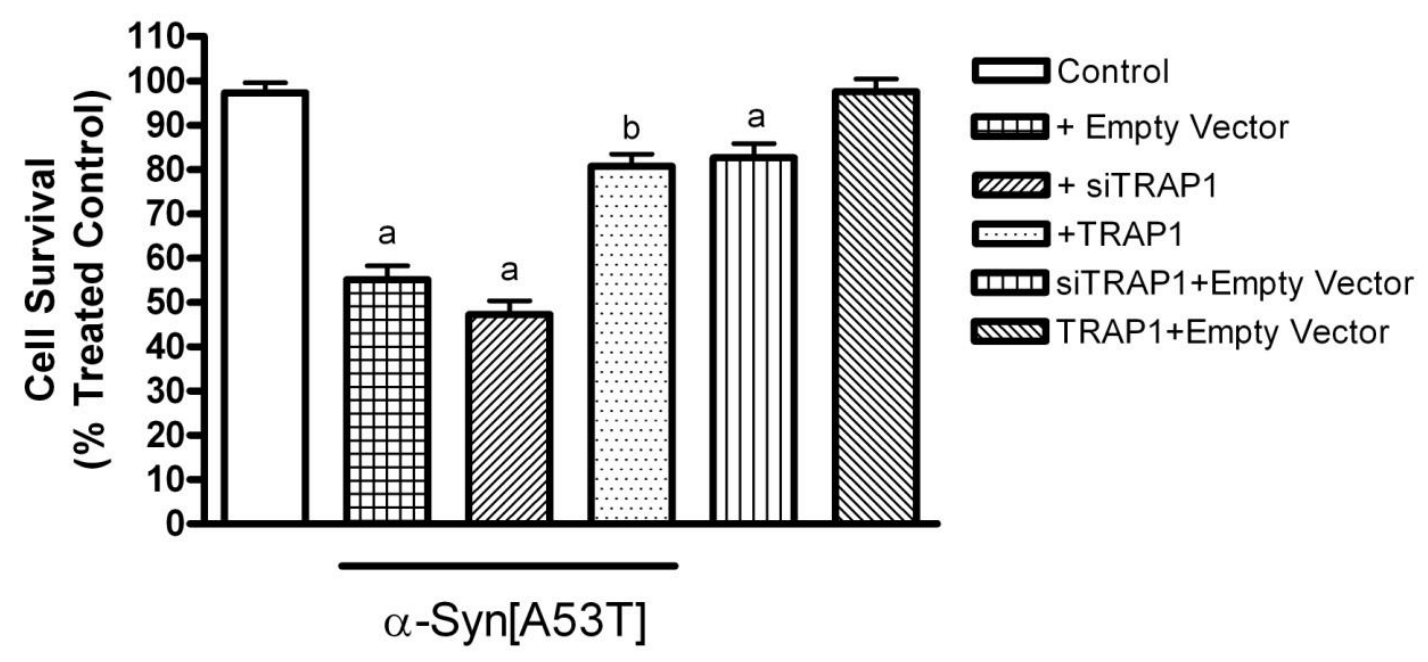

Fig. IV-28. Cell survival after hydrogen peroxide-induced oxidative stress. HEK293 cells transfected with: (left to right) empty vector and scrambled siRNA (Control); $\alpha$-Synuclein[A53T] and empty vector; $\alpha$ Synuclein[A53T] and siTRAP1; $\alpha$-Synuclein[A53T] and TRAP1; empty vector and siTRAP1; empty vector and TRAP1 were assayed for cell survival 16 hours after hydrogen peroxide $(100 \mu \mathrm{M})$ treatment. Significant changes (1-way ANOVA) are indicated (a: $\mathrm{p}<0.001$ vs. Control; b: $\mathrm{p}<0.001$ vs. $\alpha$-Synuclein[A53T] + empty vector). $\mathrm{n}=6$.

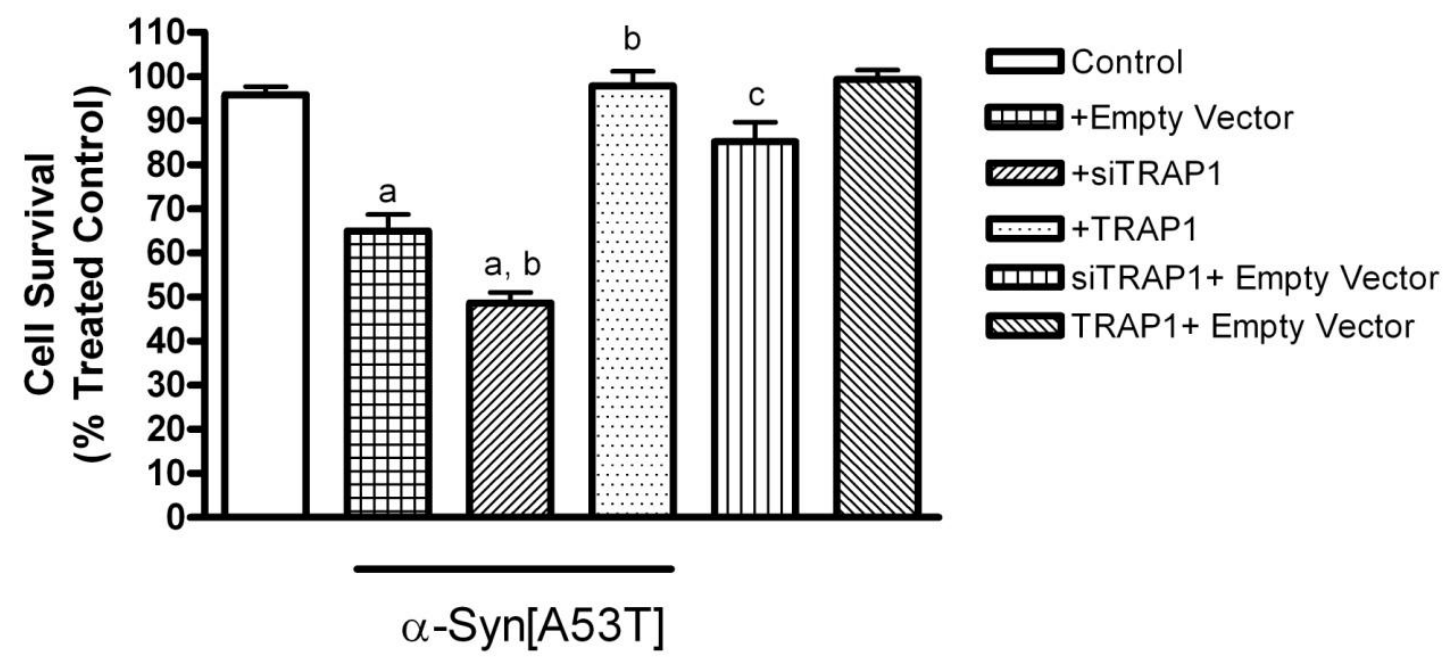

Fig. IV-29. Cell survival after rotenone-induced oxidative stress. HEK293 cells transfected with: (left to right) empty vector and scrambled siRNA (Control); $\alpha$-Synuclein[A53T] and empty vector; $\alpha$-Synuclein[A53T] and siTRAP1; $\alpha$-Synuclein[A53T] and TRAP1; empty vector and siTRAP1; empty vector and TRAP1 were assayed for cell survival 16 hours after rotenone $(200 \mu \mathrm{M})$ treatment. Significant changes (1-way ANOVA) are indicated (a: $p<0.001$ vs. Control; $b: p<0.001$ vs. $\alpha$-Synuclein[A53T] + empty vector; $c$ : $p<0.05$ vs. Control). $\mathrm{n}=6$. 


\subsubsection{5. a-Synuclein[A53T] mitochondrial toxicity: measurement of ETC complex ATP production}

Previous reports have indicated that $\alpha$-Synuclein[A53T] may interfere with mitochondrial respiration, in particular with Complex I function. ${ }^{70}$ At the same time, PINK1 knockdown or mutations in PINK1 have been show to lead to Complex I deficits in ATP production. ${ }^{239}$ The protective effect of PINK1 requires TRAP1 as a downstream effector. ${ }^{103}$ Given the rescue effect of TRAP1 on rotenone treated cells with or without $\alpha$ Synuclein[A53T] expression, it was hypothesized that the TRAP1 effect on $\alpha$ Synuclein[A53T] may in part be related to altered function of ETC components. Thus, ATP production through the ETC complexes was assayed (Complex I; Complex II; Complex IV).

Expression of $\alpha$-Synuclein[A53T] in HEK cells results in a reduction of Complex I activity, but not Complexes II or IV (Figs. IV-30-32). This reduction was enhanced by silencing TRAP1 expression. Overexpression of TRAP1 provided a rescue of the $\alpha$ Synuclein[A53T]-induced defect. In addition, activity of Complex I and II is influenced by TRAP1 alone. Overexpression of TRAP1 alone significantly enhances ATP production to above that of Control values. In contrast, siTRAP1 significantly reduced ATP production. The ATP deficit noted for $\alpha$-Synuclein[A53T] with and without siTRAP1 may therefore directly result in cellular damage as a result of insufficient ATP production for normal cellular functions such as the UPS, responsible for clearance of misfolded and toxic proteins. In addition, ATP deficit at Complex I may indicate a malfunctioning Complex, resulting in an increase of ROS production, causing downstream cell toxicity via oxidative modification of cellular contents. ${ }^{240}$ 


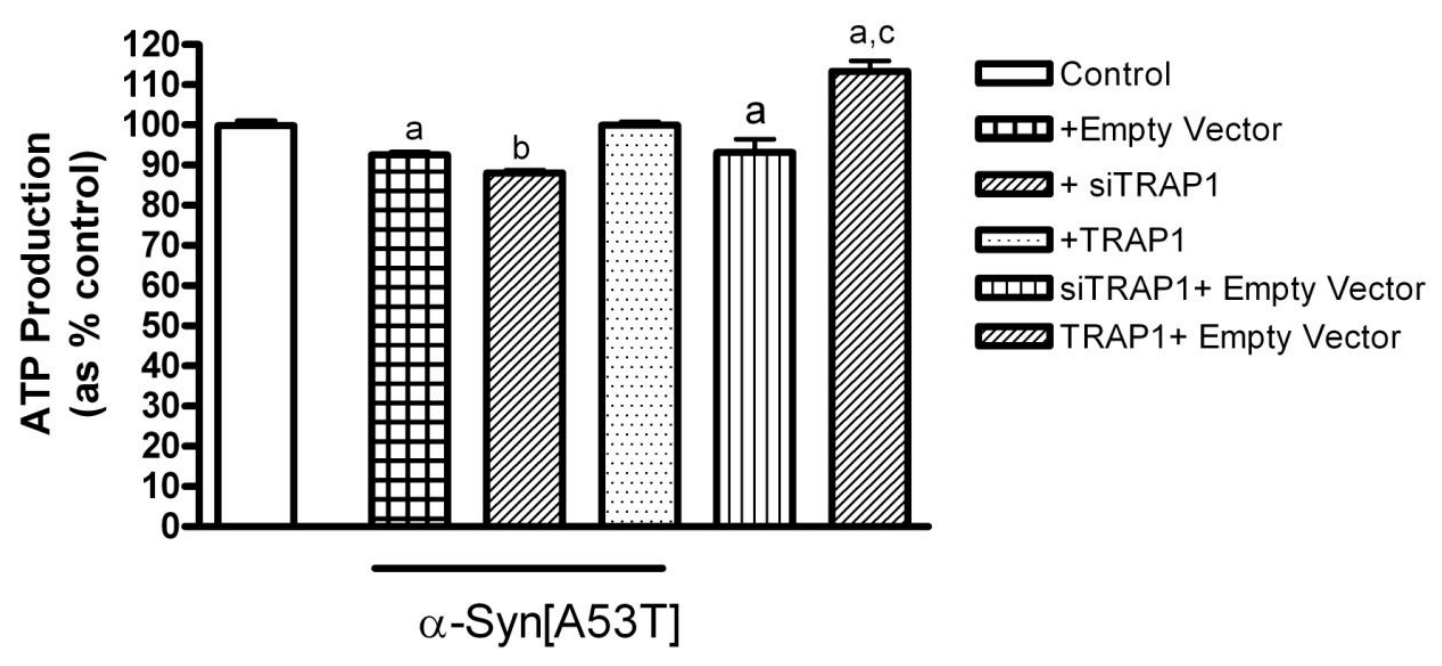

Fig. IV-30. ATP production through ETC Complex I with $\alpha$-Synuclein[A53T] $\mathbf{\pm}$ TRAP1. ATP production was measured in digitonin permeabilized cells via a luminescent assay using Complex I specific substrates. Cells were co-transfected with (left to right): empty vector and scramble siRNA (Control); $\alpha$-Synuclein[A53T] and empty vector; $\alpha$-Synuclein[A53T] and siTRAP1; $\alpha$-Synuclein[A53T] and TRAP1; empty vector and siTRAP1; empty vector and TRAP1. Significant changes (1-way ANOVA) are indicated: a: $p<0.05$ vs. Control; $b: p<0.05$ vs. $\alpha$-Synuclein[A53T]+ Empty Vector; c: $\mathrm{p}<0.05$ vs. $\alpha$-Synuclein[A53T]+ +TRAP1). $n=6-7$.

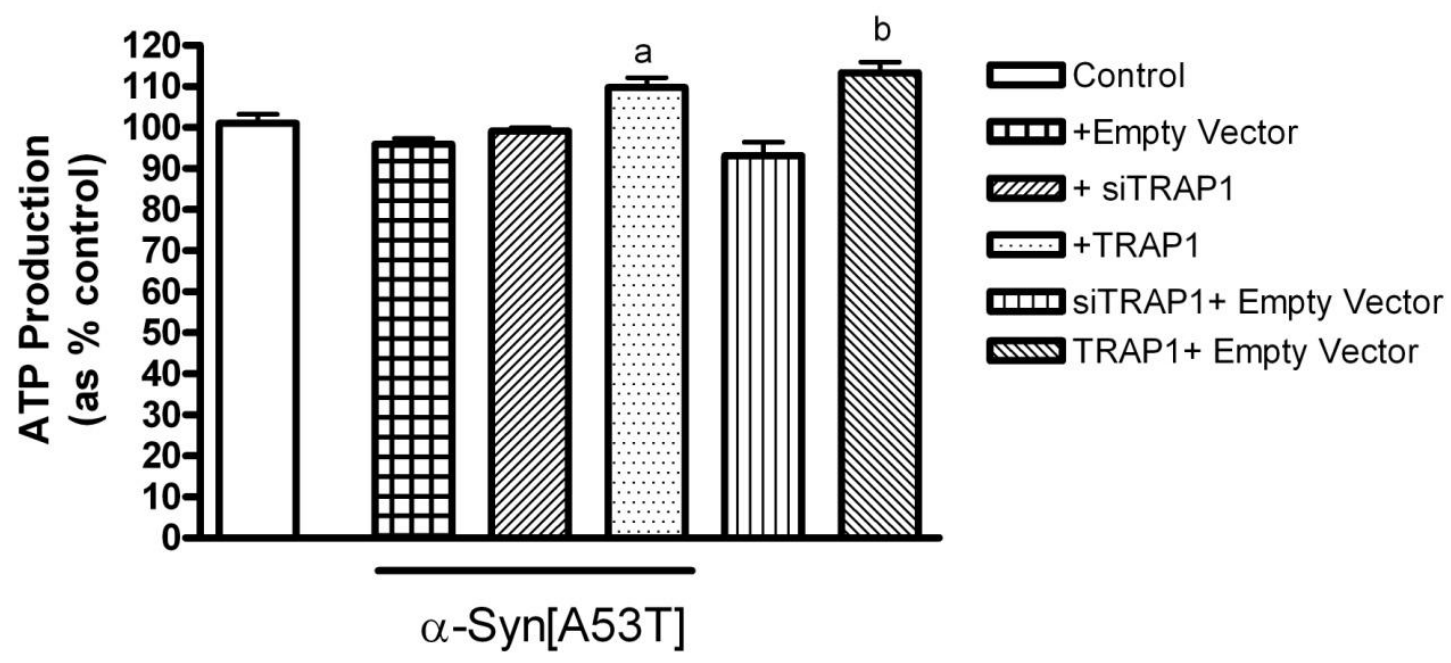

Fig. IV-31. ATP production through ETC Complex II with Synuclein [A53T] $\mathbf{\text { TRAP1}}$. ATP production was measured in digitonin permeabilized cells via a luminescent assay using Complex II specific substrates. Cells were co-transfected with (left to right): empty vector and scramble siRNA (Control); $\alpha$-Synuclein[A53T] and empty vector; $\alpha$-Synuclein[A53T] and siTRAP1; $\alpha$-Synuclein[A53T] and TRAP1; empty vector and siTRAP1; empty vector and TRAP1. Significant changes (1-way ANOVA) are indicated: a: p<0.001 vs. $\alpha$ Synuclein[A53T]+ Empty Vector; b: p<0.05 vs. Control). $n=6-7$. 


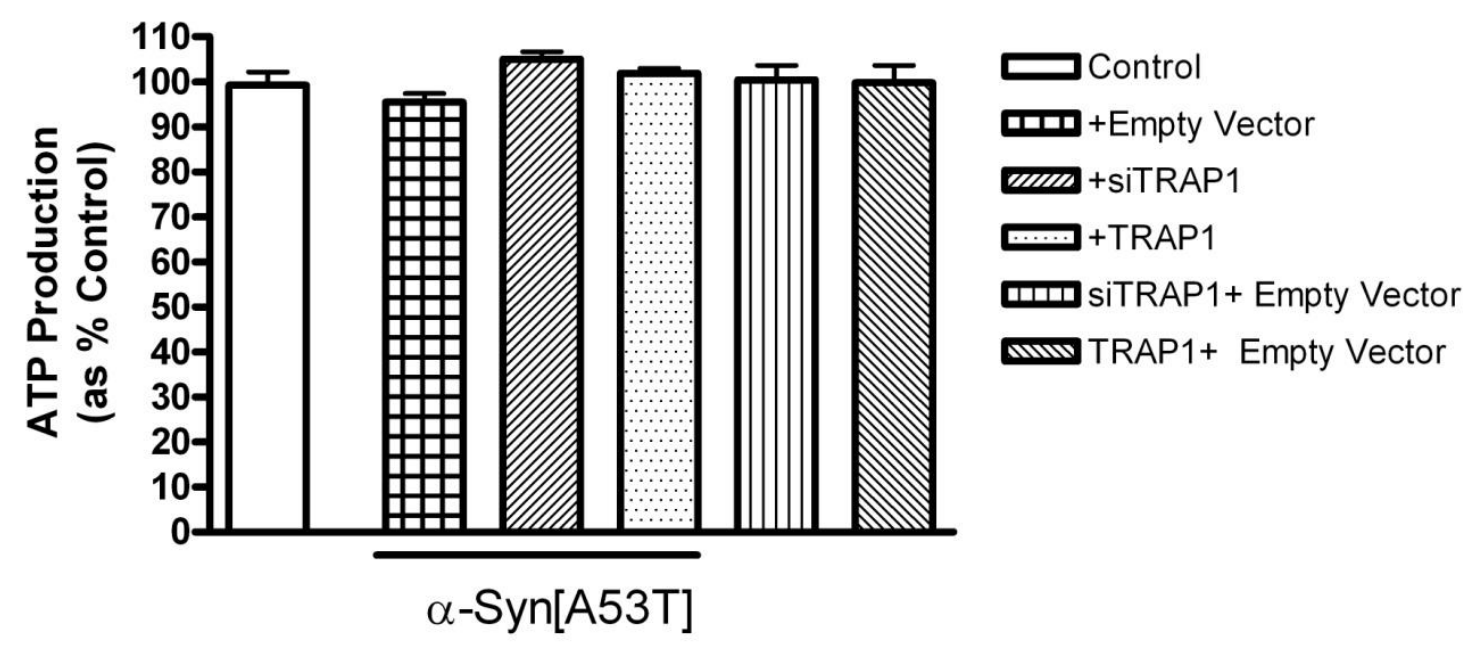

Fig. IV-32. ATP Production through ETC Complex IV in HEK293 cells expressing $\pm \alpha$-Synuclein[A53T] and \pm TRAP1. ATP production was measured in digitonin permeabilized cells via a luminescent assay using Complex IV specific substrates. Cells were co-transfected with (left to right): empty vector and scramble siRNA (Control); $\alpha$-Synuclein[A53T] and empty vector; $\alpha$-Synuclein[A53T] and siTRAP1; $\alpha$-Synuclein[A53T] and TRAP1; empty vector and siTRAP1; empty vector and TRAP1. No differences from control ATP production were observed. $n=6-8$.

\subsubsection{6. $\alpha$-Synuclein[A53T] mitochondrial toxicity: measurement of total}

\section{ATP content}

In light of the defects observed in $\alpha$-Synuclein[A53T]- induced Complex I ATP production, total ATP levels in the cell were also investigated. Only cells expressing $\alpha$ Synuclein[A53T] in combination with siTRAP1 showed a defect in total ATP. A lack of ATP might impair many cellular processes including oxidative stress response (Fig. IV-33). This suggests a possible reason for these cells enhanced sensitivity to hydrogen peroxide and in particular, rotenone, treatment. Despite Complex I inhibition noted for Synuclein[A53T] alone, overall ATP levels were unchanged. 


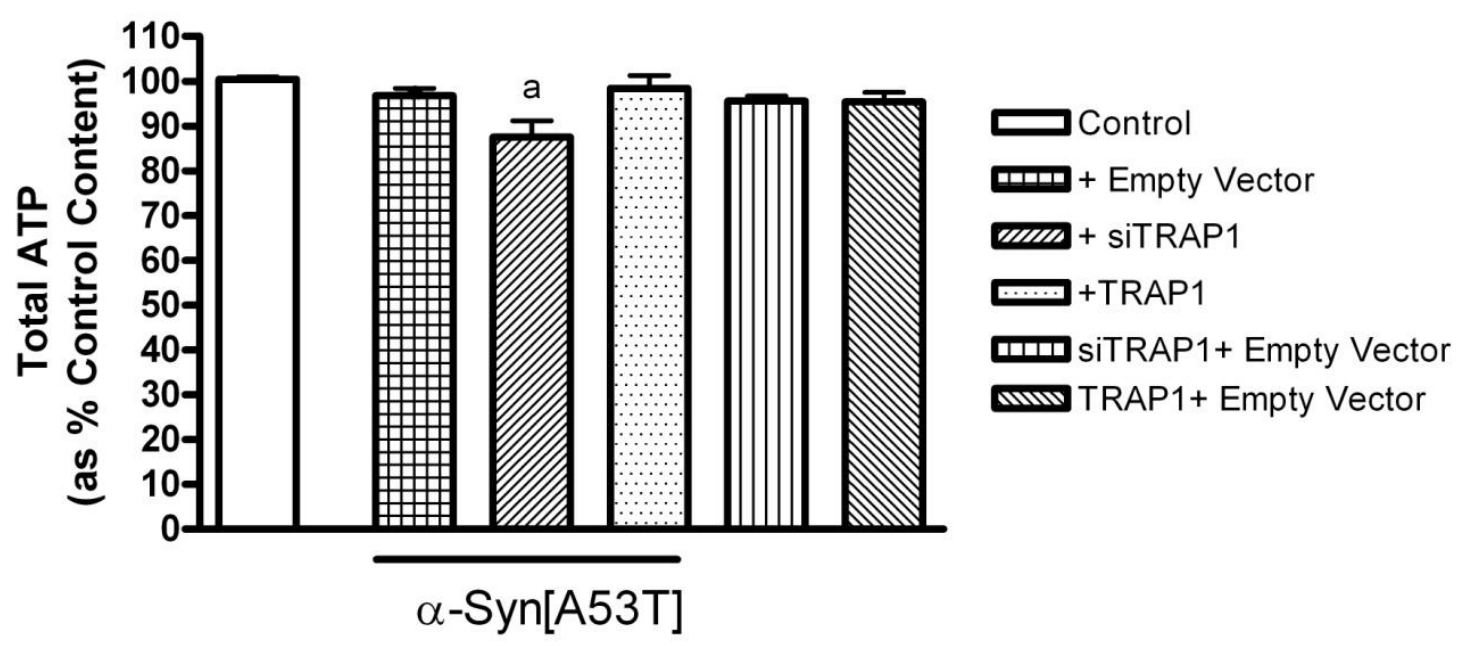

Fig. IV-33. Total cellular ATP content measured in HEK293 cells with $\pm \alpha$-Synuclein[A53T] and \pm TRAP1. Total ATP content production was measured via a luminescent assay. Cells were co-transfected with (left to right): empty vector and scramble siRNA (Control); $\alpha$-Synuclein[A53T] and empty vector; $\alpha$ Synuclein[A53T] and siTRAP1; $\alpha$-Synuclein[A53T] and TRAP1; empty vector and siTRAP1; empty vector and TRAP1. Significant changes (1-way ANOVA) are indicated: a: $\mathrm{p}<0.01$ vs. Control. $\mathrm{n}=6$.

\subsubsection{7. $\alpha$-Synuclein[A53T] mitochondrial toxicity: loss of mitochondrial membrane potential (JC-1 measurement)}

An additional aspect of mitochondrial pathology that could explain partially why $\alpha-$ Synuclein[A53T] expressing cells show an increased sensitivity to oxidative stress treatment may be due to loss of mitochondrial membrane potential. Loss of mitochondrial membrane potential predisposes cells to apoptosis. $\alpha$-Synuclein has been suggested to take on an alphahelical form that could perforate membranes. At the same time, TRAP1 protection against apoptosis has been suggested to act via inhibition of opening mitochondrial permeability transition pore (PTP), a transition that acts to inhibit oxidative phosphorylation in the mitochondria, with the downstream result of cell death. ${ }^{236}$ The mitochondrial membrane potential is thought to indirectly reflect the state of the PTP. Cells were thus assessed for mitochondrial membrane potential using the mitochondrial membrane dye, JC-1. After excitation, JC-1 emissions are depending on the mitochondrial membrane potential. Red emitted light represents polarized and green represents depolarized mitochondria and thus the changing ratio of red signal to green indicates loss of potential. Cells expressing $\alpha$ - 
Synuclein[A53T] in combination with siTRAP1 showed a loss in mitochondrial membrane potential under resting conditions and also after 4 hour rotenone $(200 \mu \mathrm{M})$ treatment (Figs. IV-34, 35), suggesting oxidative stress sensitivity is due to mitochondrial-dependent enhanced apoptotic cell death.

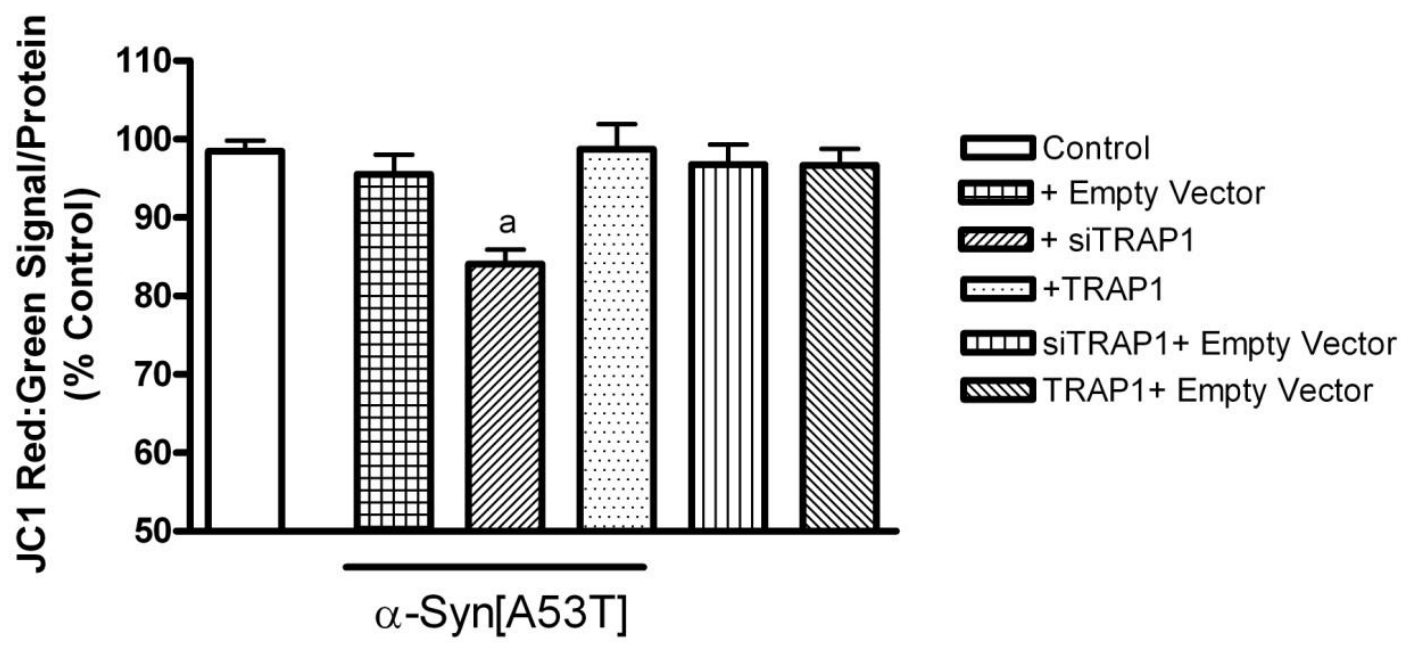

Fig. IV-34. Measurement of mitochondrial membrane potential using JC-1 in cells $\pm \alpha$-Synuclein[A53T] and \pm TRAP1 in HEK293 cells. Cells were co-transfected with (left to right): empty vector and scramble siRNA (Control); $\alpha$-Synuclein[A53T] and empty vector; $\alpha$-Synuclein[A53T] and siTRAP1; $\alpha$-Synuclein[A53T] and TRAP1; empty vector and siTRAP1; empty vector and TRAP1. Significant changes (1-way ANOVA) are indicated: a: $\mathrm{p}<0.01$ vs. Control and $\alpha$-Synuclein[A53T]. $\mathrm{n}=3$.

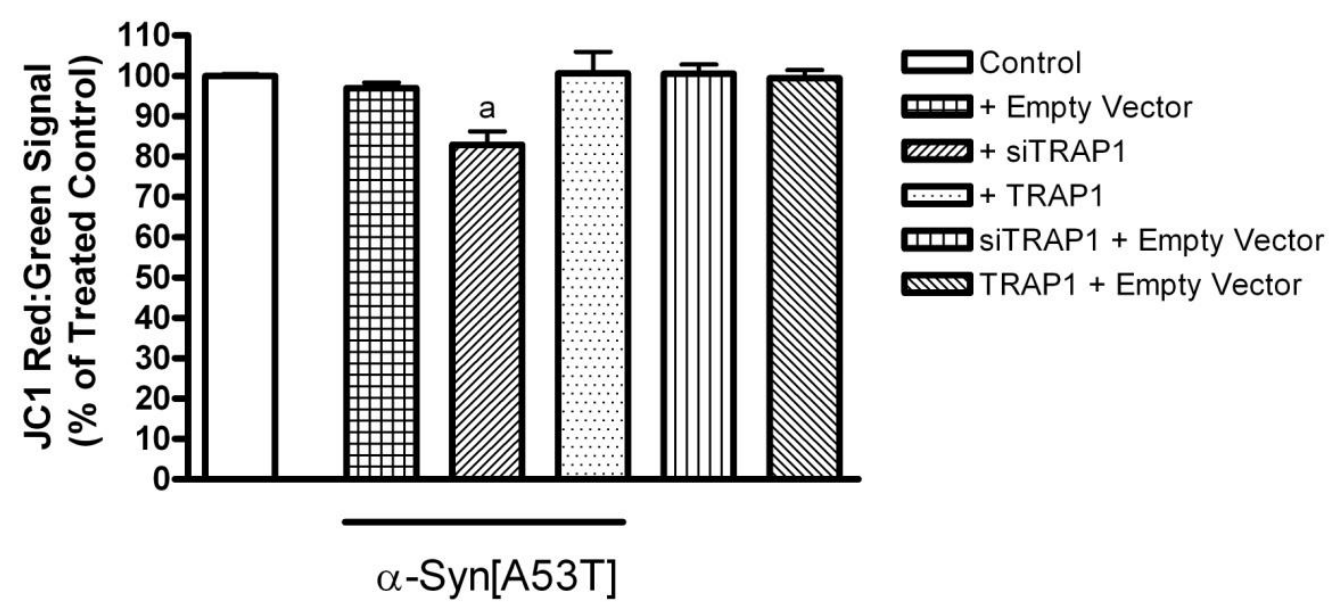

Fig. IV-35. Measurement of mitochondrial membrane potential using JC-1 in cells $\pm \alpha$-Synuclein[A53T] and \pm TRAP1 in HEK293 cells after 4 hours rotenone $(200 \mu M)$ treatment. Cells were co-transfected with (left to right): empty vector and scramble siRNA (Control); $\alpha$-Synuclein[A53T] and empty vector; $\alpha$ Synuclein[A53T] and siTRAP1; $\alpha$-Synuclein[A53T] and TRAP1; empty vector and siTRAP1; empty vector and TRAP1. Significant changes (1-way ANOVA) are indicated: a: $\mathrm{p}<0.05$ vs. Control and $\alpha$-Synuclein[A53T]. $\mathrm{n}=3$. 


\subsubsection{Measurement of total mitochondrial protein in HEK293 cells expressing $\alpha$-Synuclein[A53T] with alteration to TRAP1 expression}

Alteration in Complex I ATP production might be due to differing amount of mitochondria within the cells, this in comparison to a functional deficit in the ETC. To control for this, cell samples were probed for two proteins typically used to control for total amount of mitochondrial protein on Western blots: VDAC1 and COX4. As can be seen in Fig. IV-36, no major differences were observed for expression of VDAC1 and COX4 between samples, suggesting the decrease in Complex I ATP production results from a functional effect, not an overall decrease in mitochondrial content.

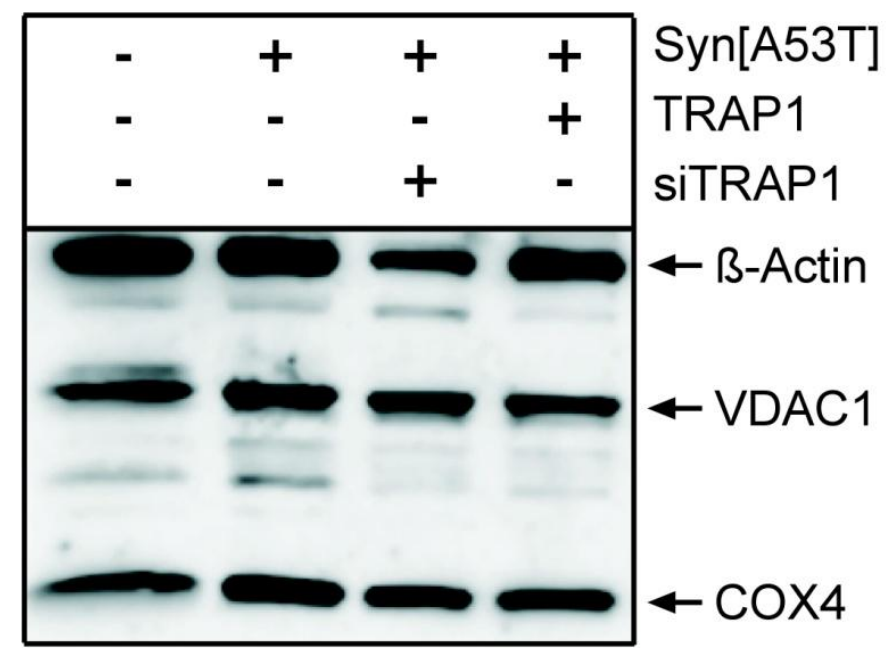

Fig. IV-36. Mitochondrial protein (VDAC1 and COX4) expression in HEK293 cells expressing $\alpha$ Synuclein[A53T] \pm TRAP1. Western blot analysis of HEK cell lysates probed with COX4 (15 kDa), VDAC1 $(31 \mathrm{kDa})$ and $\beta$-actin $(50 \mathrm{kDa})$ specific antibodies. Lanes from left to right: 1$)$ Control (empty Vector + scramble siRNA), 2) $\alpha$-Synuclein[A53T] + Empty Vector, 3) $\alpha$-Synuclein[A53T] + siTRAP1, 4) $\alpha$-Synuclein[A53T] + TRAP1. Unspecific banding seen at intermediate molecular weights. Representative blot shown from 3 experiments.

\subsubsection{Protein-protein interaction of $\alpha$-Synuclein[A53T] and TRAP1}

In light of the data indicating both entry of $\alpha$-Synuclein[A53T] into the mitochondria and the TRAP1 rescue effect, a possible direct interaction between $\alpha$-Synuclein[A53T] and TRAP1 was queried using immunoprecipitation. Protein samples from cells over-expressing both proteins were immunoprecipitated with either $\alpha$-Synuclein or TRAP1 antibodies, followed by Western blotting. Interestingly, TRAP1 Western blots showed a TRAP1 band for 
samples immunoprecipitated with $\alpha$-Synuclein, suggestive of a possible direction interaction between TRAP1 and $\alpha$-Synuclein[A53T] (Fig. IV-37). However, the reverse result, that being a TRAP1 IP showing a band on a $\alpha$-Synuclein Western could not be demonstrated. It is possible that the interaction of Synuclein-TRAP1 with the TRAP1 antibody in use under IP conditions was not sufficient for a pull-down. However, due to the lack of additional, working TRAP1 antibodies, this hypothesis cannot be confirmed.

\section{specific antibody used for IP}

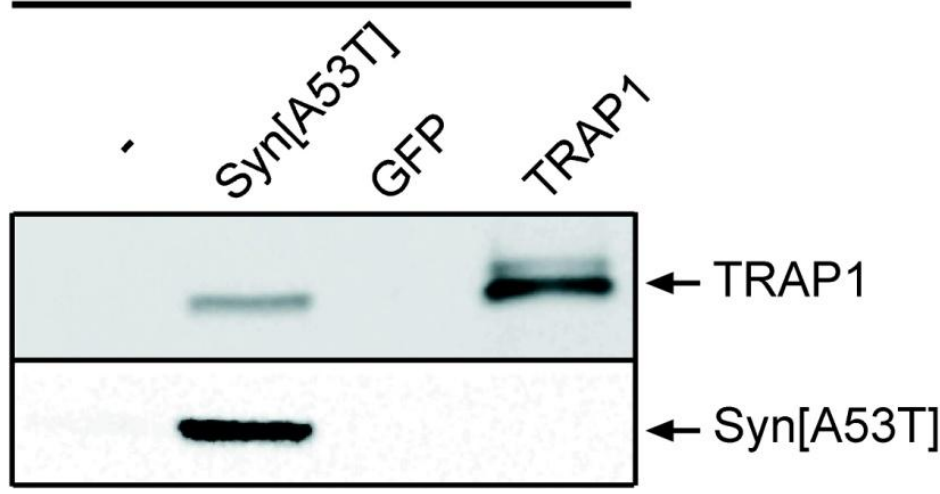

Fig. IV-37. Protein-protein interaction between TRAP1 and $\alpha$-Synuclein[A53T]. IP with $\alpha$-Synuclein antibody followed by TRAP1 Western resulted in a TRAP1 (75 kDa) band on TRAP1 Western blot. Top blot for TRAP1, bottom blot for $\alpha$-Synuclein. Lanes from left to right: 1) IP without antibody, 2) IP with Synuclein antibody, 3) IP with GFP antibody, 4) IP with TRAP1 antibody. Slight non-specific banding at $76 \mathrm{kDa}$ often seen when large amounts of TRAP1 present in gel. Blot representative of 3 experiments.

\subsubsection{Creation of TRAP1 mutants and expression in HEK293 cells}

In order to better understand the mechanism by which TRAP1 is able to exert its protective effect against $\alpha$-Synuclein-induced toxicity, a number of TRAP1 mutants were produced. In particular, given that the PINK1 protective effect is dependent upon TRAP1 phosphorylation, it was therefore of interest to ask the same question for $\alpha$-Synuclein rescue: is TRAP1 phosphorylation necessary for $\alpha$-Synuclein rescue? In addition, TRAP1 is defined as an HSP90-like chaperone protein possessing an ATPase domain. Thus, the second question was: will TRAP1 rescue if its ATPase function has been abolished?

The exact sites for TRAP1 phosphorylation were not yet experimentally defined. Thus, 2 putative phosphorylation sites (based on phospho-site data available at time) were 
selected and mutated using in vitro mutagenesis: changing threonine at amino acid position 494 to alanine [T494A] and tyrosine at 498 to glycine [Y498G]. The human TRAP1 ATPase domain shares high homology with both other HSP90 proteins and other species TRAP1 sequences (for protein sequence data, see Appendix Methods Figures A1-1-3). Previously published results from yeast indicated that mutation of specific amino acid in the ATPase domain stopped binding of ATP. ${ }^{241}$ This amino acid was highly conserved in both HSP90 and TRAP1 proteins and therefore a new mutant with aspartic acid at position 148 changed to asparagine was created (TRAP1[D158N]). Expression of the new constructs within HEK293 cells was confirmed (Fig. IV-38). Expression of TRAP1, whether wt or mutant, resulted in similar overexpression, both with and without coincident $\alpha$-Synuclein[A53T] expression.

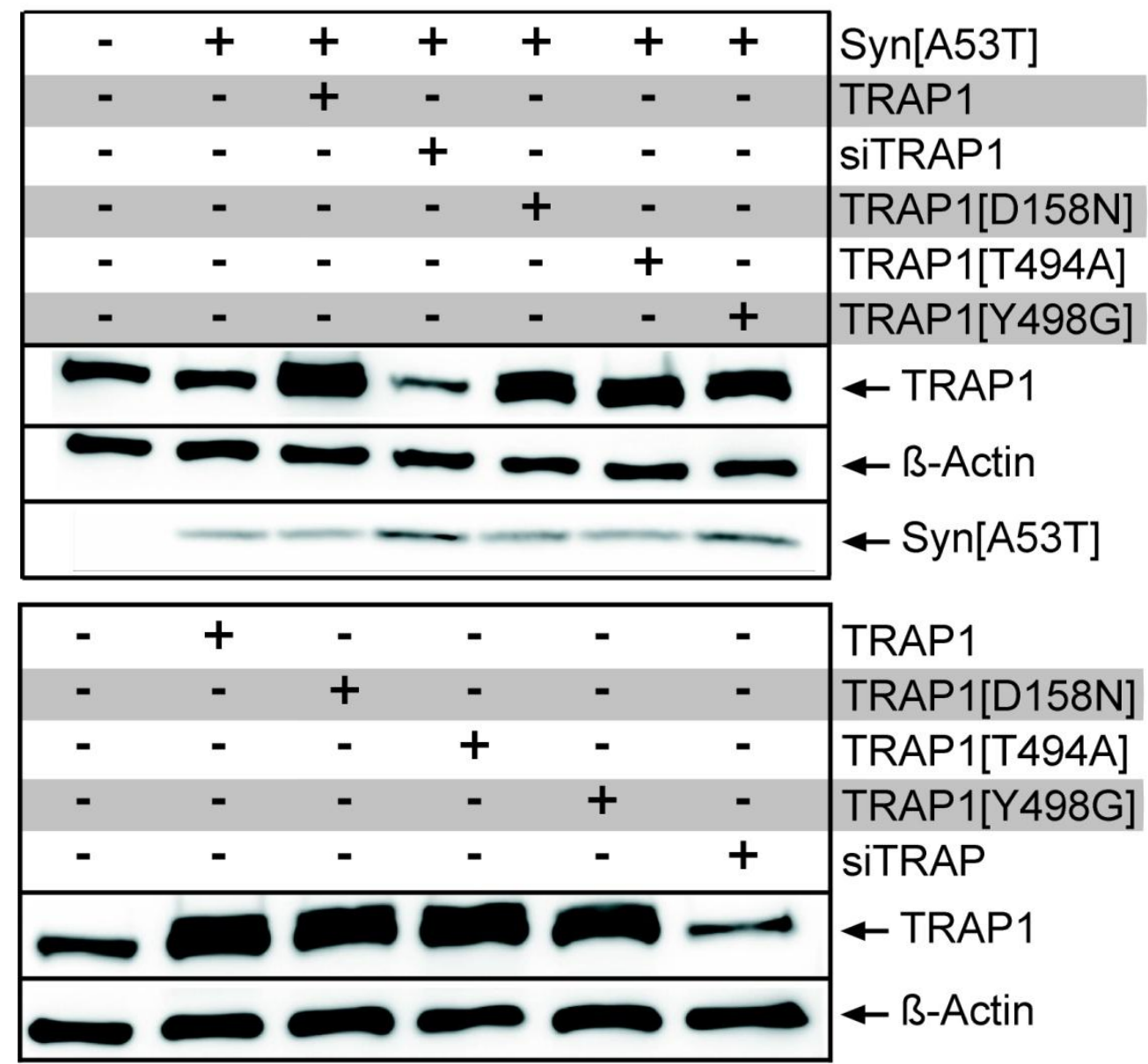

Fig. IV-38. Expression of TRAP1 mutants and $\boldsymbol{\alpha}$-Synuclein[A53T] in HEK293 cells. After transfection with indicated expression constructs, HEK293 lysates were used for Western blot analysis and probed with TRAP1 (75 kDa), $\alpha$-Synuclein $(16 \mathrm{kDa})$ and $\beta$-Actin $(50 \mathrm{kDa}$ protein loading control) specific antibodies. Top panel lanes from left to right: 1) Control (empty vector+ scramble siRNA), 2) $\alpha$-Synuclein[A53T]+ Empty Vector, 3) $\alpha$ Synuclein[A53T]+ TRAP1, 4) $\alpha$-Synuclein[A53T]+ siTRAP1, 5) $\alpha$-Synuclein[A53T]+ TRAP1 [D158N], 6) $\alpha-$ Synuclein[A53T]+ TRAP1 [T494A], 7) $\alpha$-Synuclein[A53T]+ TRAP1 [Y498G]. Bottom panel shows TRAP1 
expression in the absence of $\alpha$-Synuclein[A53T] expression. Lanes from left to right: 1) Control (empty vector+ scramble siRNA), 2) Empty Vector+ TRAP1, 3) Empty Vector+ TRAP1 [D158N], 4) Empty Vector+ TRAP1 [T494A], 5) Empty Vector+ TRAP1 [Y498G], 6) Empty Vector+ siTRAP1. Representative blots from 3 experiments shown.

\subsubsection{Phosphorylation state of TRAP1 and TRAP1 mutants}

The phosphorylation of TRAP1 might be crucial to its protective function. Therefore the phosphorylation status of TRAP1 was both under resting and oxidative stress conditions was analyzed by IP followed by Western blot analysis, probing with phosphoserine/threonine/tyrosine specific antibodies.
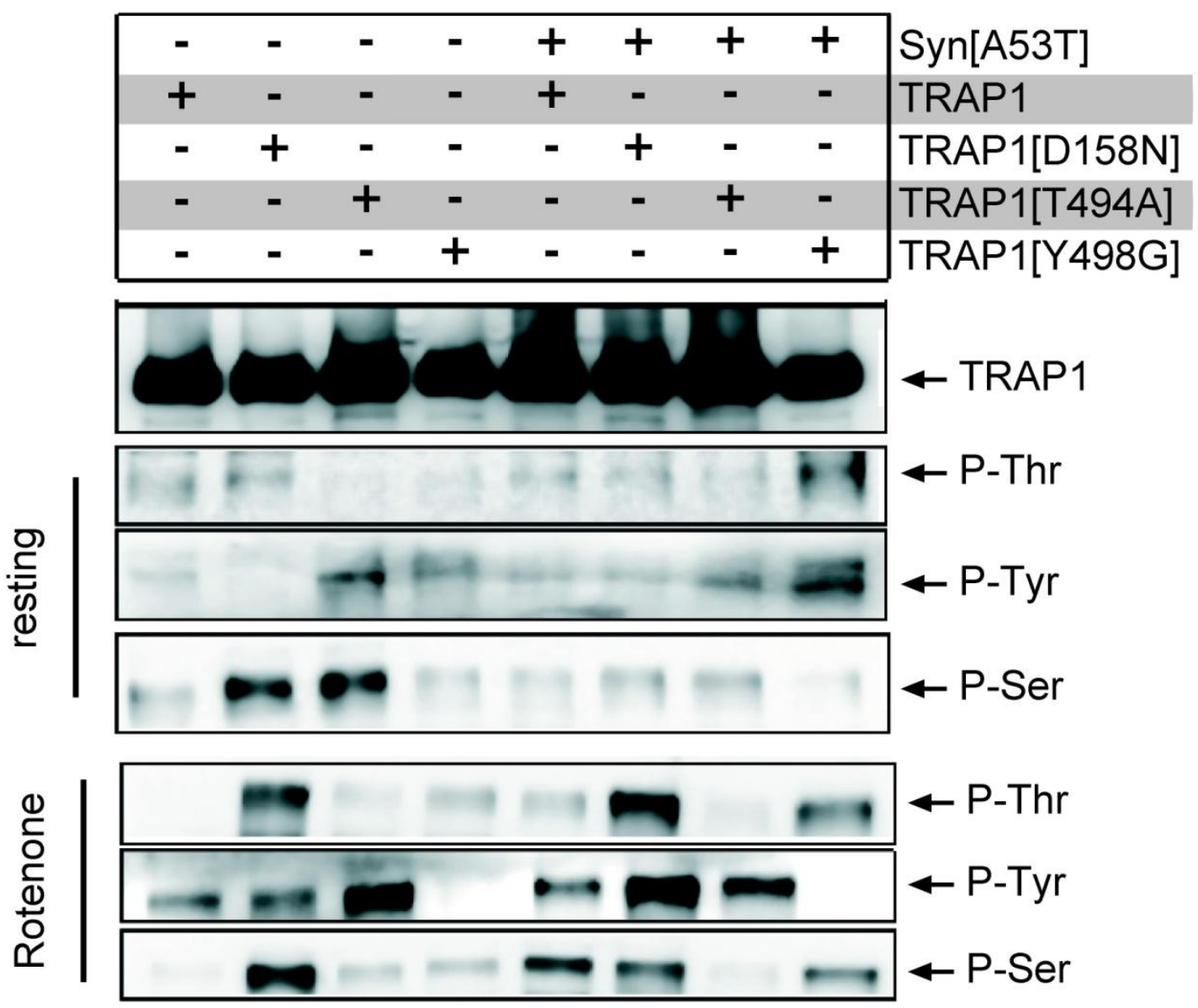

Fig. IV-39. Phosphorylation state of wt TRAP1 and TRAP1 mutants in HEK293 cells. Western blot analysis of HEK cells after IP of TRAP1 $(75 \mathrm{kDa})$, under both resting and oxidative stress $(200 \mu \mathrm{M}$ rotenone for 4 hours $)$ conditions. Blots were probed for TRAP1, phospho-threonine, phospho-tyrosine and phospho-serine using specific antibodies. Different TRAP1 variants were expressed in absence of $\alpha$-Synuclein[A53T] (lanes 1-4) or in combination with $\alpha$-Synuclein[A53T] expression (lanes 5-8). Samples from left to right: 1) wt TRAP1, 2) TRAP1[D158N], 3) TRAP1[T494A], 4) TRAP1[Y498G]. 5-8 repeat. Control IPs with GFP did not show Western blot TRAP1 staining (not shown). Representative blots shown.

Under resting conditions, wt TRAP1 appears to be lightly phosphorylated at threonine and serine (Fig. IV-39). After oxidative stress, wt TRAP1 evidenced tyrosine and serine 
phosphorylation, with particular enhancement of both with $\alpha$-Synuclein[A53T] co-expression. Co-expression of $\alpha$-Synuclein[A53T] differentially affected serine phosphorylation dependent on TRAP1 variant. The [T494A] mutant showed minimal evidence of threonine phosphorylation under both tested conditions. Thus, mutation was successful in eliminating the major threonine phosphorylation site. In comparison to wt, the [T494A] mutant showed enhanced tyrosine phosphorylation under both conditions. In contrast to wt, serine phosphorylation was enhanced in the absence of $\alpha$-Synuclein[A53T], especially under resting conditions. [Y498G] showed only faint tyrosine phosphorylation at rest, with an increase when $\alpha$-Synuclein[A53T] is co-expression. Tyrosine phosphorylation of [Y498G] after rotenone treatment was not observed. Thus, it is seems likely that sites other than Y498 are available for tyrosine phosphorylation sites are found in TRAP1 and that their phosphorylation may be connected to TRAP1 function/response to $\alpha$-Synuclein[A53T]. Similar to wt TRAP1, the extent of threonine and serine phosphorylation on [Y498G] during oxidative stress was dependent on the presence of $\alpha$-Synuclein[A53T]. In contrast to wt and other mutants, the [D158N] mutant showed primarily serine phosphorylation at rest and heavy phosphorylation of all types when under oxidative stress. In summary, phosphorylation indeed appears to play a role in TRAP1 function ${ }^{103}$, with differential phosphorylation dependent on either co-expression of $\alpha$-Synuclein[A53T] or oxidative stress treatment.

\subsubsection{Effect of TRAP1 mutation on protein-protein interaction with $\alpha$ - Synuclein[A53T]}

Effect of TRAP1 mutation on the observed protein-protein interaction with $\alpha$ Synuclein[A53T] was investigated. IP from HEK293 lysates variously expressing $\alpha$ Synuclein[A53T] in combination with the different TRAP mutations both before and after exposure to rotenone oxidative stress was performed in analogy to previously described experiments (see 4.3.2.9.). Precipitates were investigated for co-precipitation of TRAP1 in 
Western blot analysis. All mutants, in particular, TRAP1[D158N],seem to have decreased interaction with $\alpha$-Synuclein[A53T] as compared with wt TRAP1 protein (Fig. IV-40).

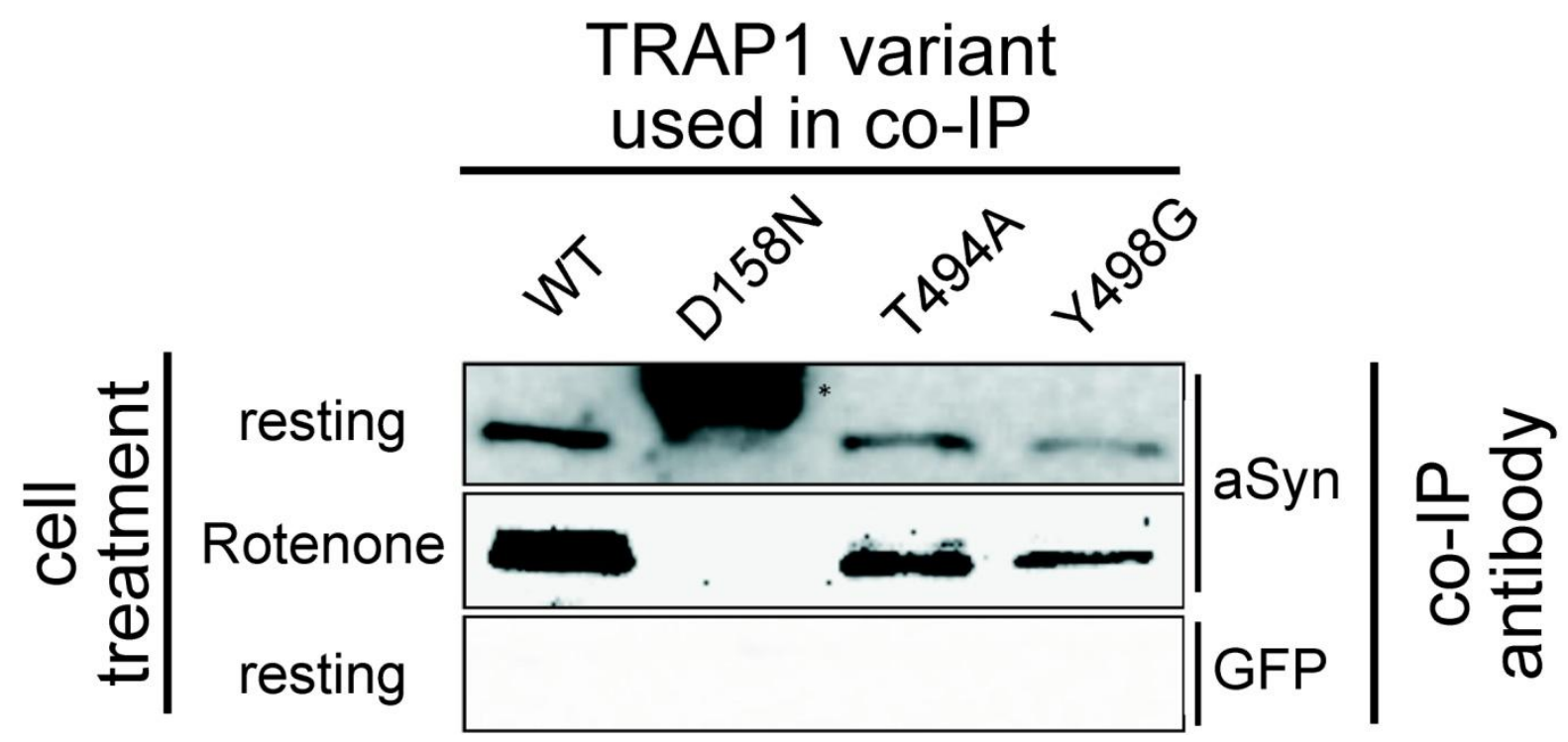

Fig. IV-40. TRAP1 mutants protein-protein interaction with $\alpha$-Synuclein[A53T] from HEK293 lysates (IP). Lysates of HEK cells co-expressing $\alpha$-Synuclein[A53T] in combination with the different TRAP1 variants were collected both before and after 4 hour $(100 \mathrm{uM})$ rotenone treatment. Lysates were then used for IP with a $\alpha$ Synuclein specific antibody. Precipitates were separated using SDS-PAGE and blotted. Western blot were analyzed for co-precipitation of TRAP1 using a TRAP1 specific antibody (75 kDa). Lanes from left to right: 1) wt TRAP1, 2) TRAP1[D158N], 3) TRAP1[T494A], 4) TRAP1[Y498G]. Control IPs with GFP (lower panel) did not show Western blot TRAP1 staining (rotenone treated GFP IP not shown; also had no banding). Resting conditions IPs of TRAP1[D158N] consistently showed high molecular weight band of unknown significance (asterisk). Representative blots shown.

\subsubsection{Effect of TRAP1 mutation on TRAP1 oxidative stress rescue effect}

Alteration to the TRAP1 rescue effect for oxidative stress was then investigated for cells expressing $\alpha$-Synuclein[A53T] and mutant TRAP1. Cells were treated for 16 hours with either hydrogen peroxide or rotenone. Compared to wt TRAP1, expression of the TRAP1 mutants [D158N], [T494A] and [Y498G] alone resulted in a significant decrease in survival after oxidative stress treatment, independent of the stress inducer (Fig. IV-41). In combination with $\alpha$-Synuclein[A53T], the observed rescue effect by wt TRAP1 trended to be reduced by the TRAP1 mutants, however the observed differences were mainly not significant. Only under rotenone treatment, cells co-expressing $\alpha$-Synuclein[A53T] in combination with the ATPase deficient mutant TRAP1[D158N] displayed a significant reduction on cell survival. 


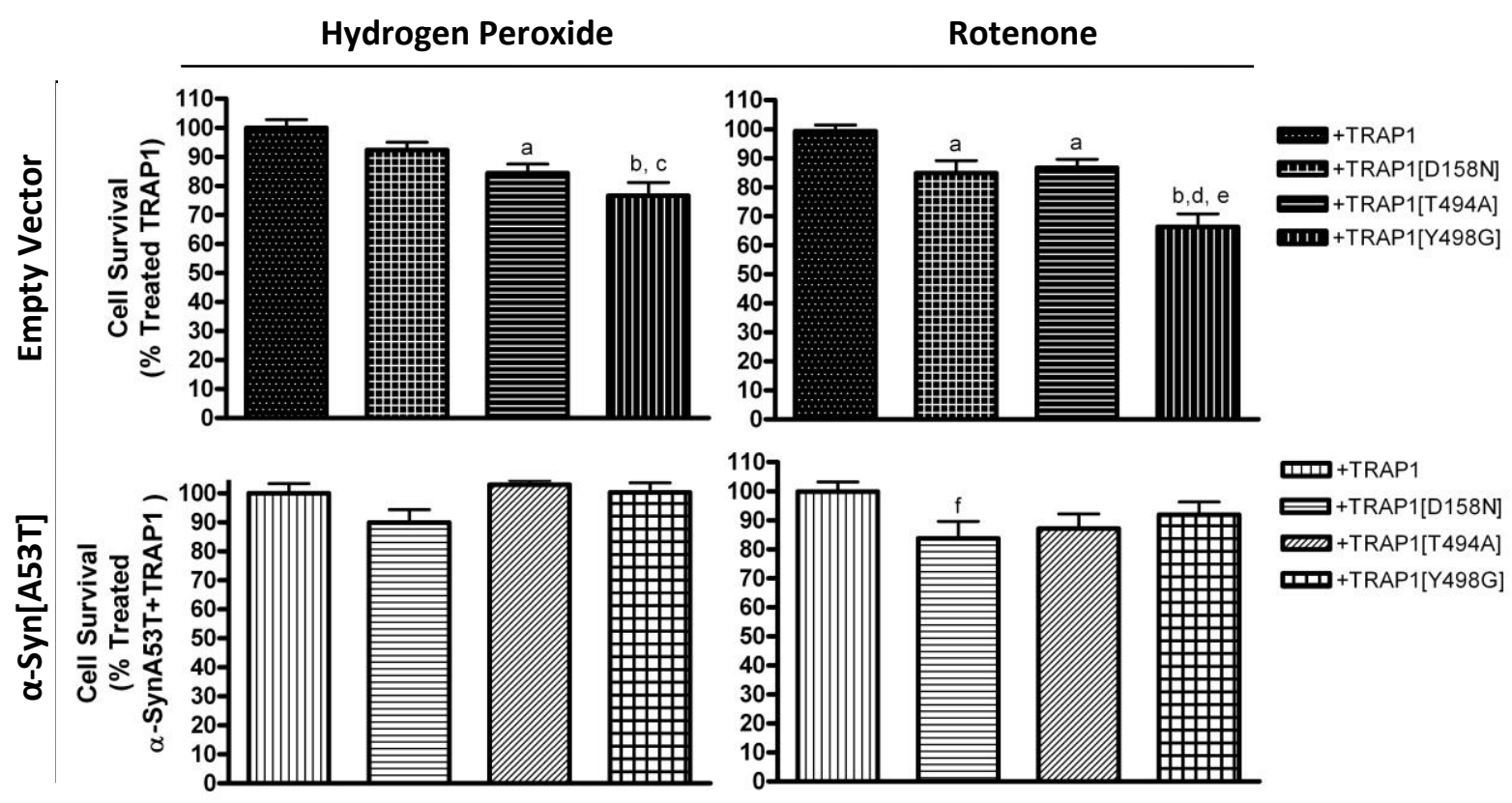

Fig. IV-41. Cell survival after oxidative stress treatment of HEK293 cells expressing wt TRAP1 or mutant TRAP1 in presence/absence of $\boldsymbol{\alpha}$-Synuclein[A53T]. HEK293 cells transfected with: (top panels, left to right) empty vector and TRAP1; empty vector and TRAP1[D158N]; empty vector and TRAP1[T494A]; empty vector and TRAP1[Y498G], (bottom panels, left to right): $\alpha$-Synuclein[A53T] and TRAP1; $\alpha$-Synuclein[A53T] and TRAP1[D158N]; $\alpha$-Synuclein[A53T] and TRAP1[T494A]; $\alpha$-Synuclein[A53T] and TRAP1[Y498G]; were assayed for cell survival $16 \mathrm{~h}$ after either hydrogen peroxide $(100 \mu \mathrm{M})$ or rotenone $(200 \mu \mathrm{M})$ treatment. Significant changes (1-way ANOVA) are indicated: $a: p<0.05$ vs. empty vector+ TRAP1; $b$ : $p<0.001$ vs. empty vector+ TRAP1; c: p<0.01 vs. Empty vector+ TRAP1[D158N]; d: p<0.001 vs. empty vector+ TRAP1 [D158N]; e: $p<0.01$ vs. empty vector+ TRAP1 [T494A]; : p<0.05 vs. $\alpha$-Synuclein[A53T]+ TRAP1. $n=6$ for empty vector +TRAP1, $\mathrm{n}=3$ for TRAP1 mutants.

\subsubsection{Effect of TRAP1 mutations on ATP production of ETC complexes}

Similar to the previous experiment, HEK cells were transfected with either wt TRAP1 or TRAP1 mutants in absence or presence of $\alpha$-Synuclein[A53T] co-expression. Two days post-transfection ATP production by the different ETC was analyzed. First, ATP-production by complex I was measured (Fig. IV-42). In this assay, TRAP1[D158N] and [T494A]expressing cells showed a decrease in ATP-production as compared to wt TRAP1. This decrease was more pronounced in cells co-expressing $\alpha$-Synuclein[A53T], as compared to cells without $\alpha$-Synuclein[A53T].

Next, ATP-production by complex II was analyzed using the same paradigm (Fig. IV43). In this analysis, in the absence of $\alpha$-Synuclein[A53T] expression, no significant changes 


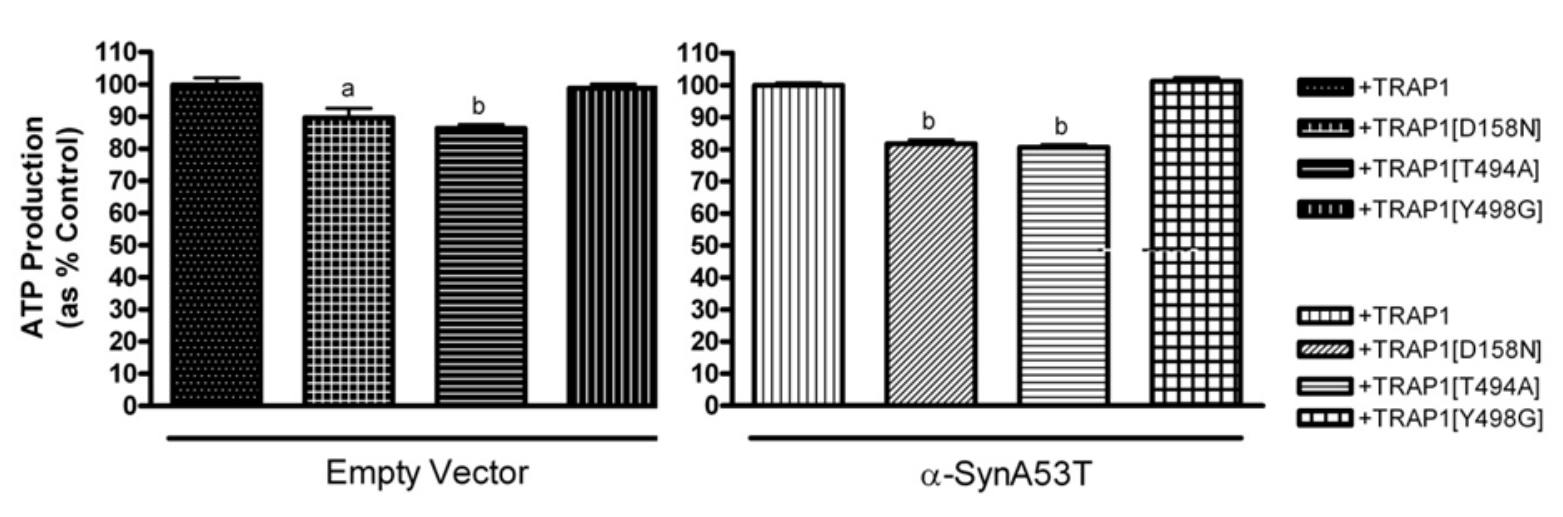

Fig. IV-42. Effect of TRAP1 mutation on ATP production via Complex I in HEK293 cells. TRAP1 overexpression previously shown to enhance ATP production via Complex I. HEK293 cells transfected with: (left panel, left to right) empty vector and TRAP1(Control); empty vector and TRAP1[D158N]; empty vector and TRAP1[T494A]; empty vector and TRAP1[Y498G]; (right panel, left to right) $\alpha$-Synuclein[A53T]and TRAP1(Control); $\alpha$-Synuclein[A53T] and TRAP1[D158N]; $\alpha$-Synuclein[A53T] and TRAP1[T494A]; $\alpha-$ Synuclein[A53T] and TRAP1[Y498G]; were assayed for Complex I ATP production. Significant changes (1way ANOVA) vs. Control are indicated: a: $\mathrm{p}<0.01$ vs. Control; $b$ : $\mathrm{p}<0.001$ vs. Control. In addition, both empty vector+TRAP1[D158N], TRAP1[T494A] were significantly different than empty vector+ TRAP1[Y498G] $(\mathrm{p}<0.01$ and $\mathrm{p}<0.001$ respectively). $\alpha$-Synuclein[A53T]+ TRAP1[D158N], TRAP1[T494A] were significantly different than $\alpha$-Synuclein[A53T]+ TRAP1[Y498G] ( $\mathrm{p}<0.001$ for both). Left graph ATP production normalized to Control $=$ empty vector + TRAP1; right graph, Control $=\alpha$-Synuclein[A53T] + TRAP1. $n=6$ for + TRAP1, $n=3$ for TRAP1 mutants.
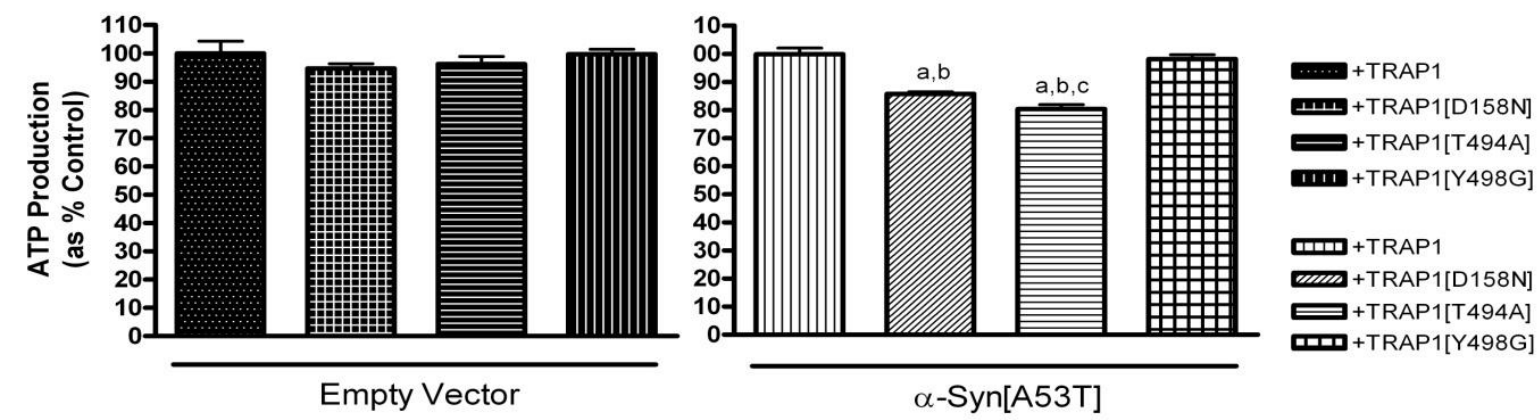

Fig. IV-43. Effect of TRAP1 mutation on ATP production via Complex II ATP production in HEK293 cells. wt TRAP1 overexpression previously shown to enhance ATP production via Complex II in both presence and absence of $\alpha$-Synuclein[A53T]. HEK293 cells transfected with: (left panel, left to right) empty vector and TRAP1 (Control); empty vector and TRAP1[D158N]; empty vector and TRAP1[T494A]; empty vector and TRAP1[Y498G]; (right panel, left to right): $\alpha$-Synuclein[A53T] and TRAP1 (Control); $\alpha$-Synuclein[A53T] and TRAP1[D158N]; $\alpha$-Synuclein[A53T] and TRAP1[T494A]; $\alpha$-Synuclein[A53T] and TRAP1[Y498G]; were assayed for Complex II ATP production. Significant changes (1-way ANOVA) to Control observed: a: $\mathrm{p}<0.001$ vs. Control. $\alpha$-Synuclein[A53T]+ [D158N] was also significantly different than $\alpha$-Synuclein[A53T] + TRAP1 [Y498G] $(\mathrm{p}<0.001) . \alpha$-Synuclein[A53T]+ [T494A] was significantly different than both other mutants $(\mathrm{p}<0.05$ and $\mathrm{p}<0.001$ respectively). Left graph ATP production Control= empty vector + TRAP 1 ; right graph Control $=\alpha-$ Synuclein[A53T]+ TRAP1. $n=6$ for +TRAP1 and, $n=3$ for TRAP1 mutants.

in ATP production by complex II could be detected. However, similar to the situation observed for complex I, TRAP1[D158N] and TRAP1[T498G] displayed reduced ATPproduction by complex II if co-expressed with $\alpha$-Synuclein[A53T]. An entirely different situation was observed if ATP-production of complex IV was analyzed (Fig. IV-44). Cells without $\alpha$-Synuclein[A53T] expression showed significantly reduced ATP-production with all 
TRAP1 mutant variants. Compared to wt TRAP1-expressing cells, the decrease in ATP was most prominent for cells expressing TRAP1[T494A]. In cells with $\alpha$-Synuclein[A53T] and TRAP1 expression, however, only TRAP1[T494A] co-expressing cells showed significant reduced ATP-production compare to wt TRAP1.

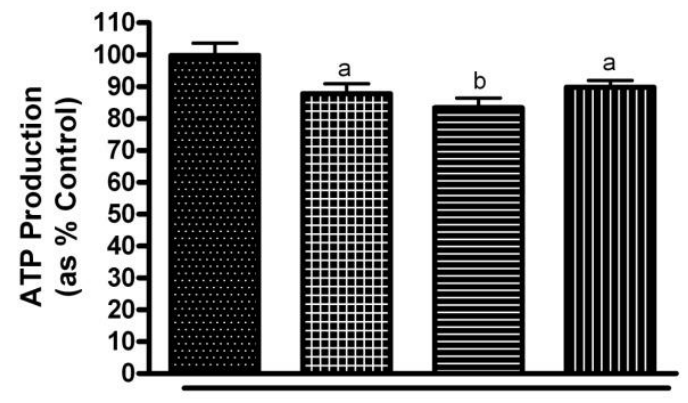

Empty Vector

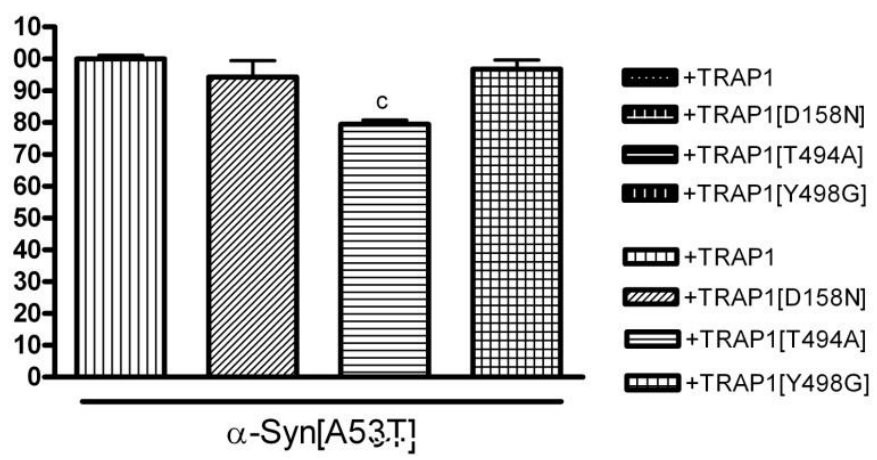

Fig. IV-44. Effect of TRAP1 mutation on ATP production via Complex IV in HEK293 cells. Overexpression of wt TRAP1 was previously shown not to affect Complex IV ATP production. HEK293 cells transfected with: (left panel, left to right) empty vector and TRAP1 (Control); empty vector and TRAP1[D158N]; empty vector and TRAP1[T494A]; empty vector and TRAP1[Y498G]; (right panel, left to right): $\alpha$-Synuclein[A53T] and TRAP1 (Control); $\alpha$-Synuclein[A53T] and TRAP1[D158N]; $\alpha$-Synuclein[A53T] and TRAP1[T494A]; $\alpha$-Synuclein[A53T] and TRAP1[Y498G]; were assayed for Complex IV ATP production. Significant changes (1-way ANOVA) vs. Control observed: a: $p<0.05$ vs. Control; b: $p<0.01$ vs. Control; c: $\mathrm{p}<0.001$ vs. Control. In addition, $\alpha$-Synuclein[A53T] and TRAP1[T494A] was significantly different than both other mutants $(\mathrm{p}<0.001$ for both). Left graph ATP production Control= empty vector+ TRAP1; right graph Control $=\alpha$-Synuclein[A53T] + TRAP1. $n=6+$ TRAP1, $n=3$ for TRAP1 mutants.

\subsubsection{Effect of TRAP1 mutation on mitochondrial membrane potential}

The impaired ATP production by the different complexes might suggest that TRAP1mutations might have an effect on the mitochondrial membrane potential. As the proton gradient across the inner mitochondrial membrane is used for ATP production by ATPsynthase, alterations of the membrane potential might explain previously measured differences in ATP. Therefore, the mitochondrial membrane potential of the TRAP1 mutants was compared to that of wt TRAP1, when expressed alone or in combination with $\alpha$ Synuclein[A53T]. However, no significant differences from wt TRAP1 were observed (Fig. IV-45). 


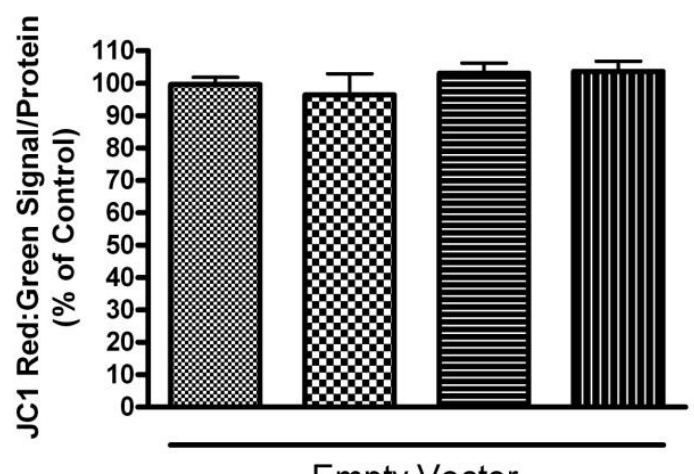

Empty Vector

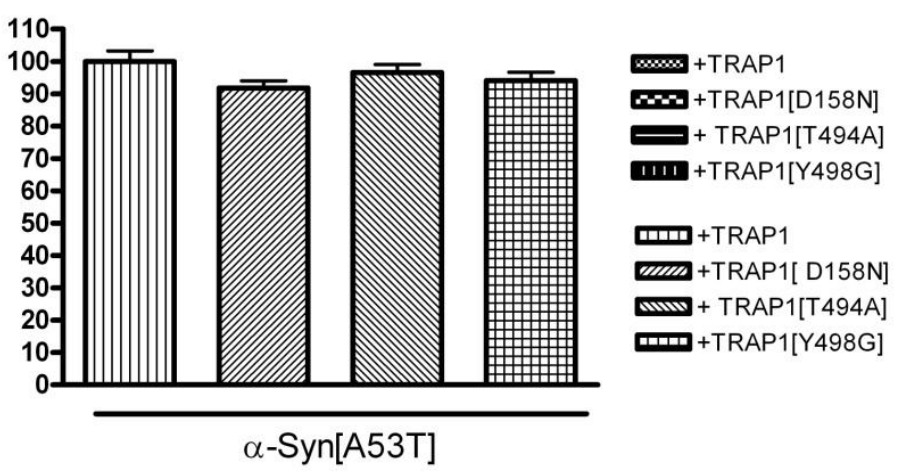

Fig. IV-45. Measurement of mitochondrial membrane potential in HEK293 cells expressing wt TRAP1 or TRAP1 mutants. JC-1 measurements with TRAP1 overexpression was previously shown to be no different than control cells. HEK293 cells transfected with: (left panel, left to right) empty vector and TRAP1 (Control); empty vector and TRAP1[D158N]; empty vector and TRAP1[T494A]; empty vector and TRAP1[Y498G]; (right panel, left to right): $\alpha$-Synuclein[A53T] and TRAP1 (Control); $\alpha$-Synuclein[A53T] and TRAP1[D158N]; $\alpha$ Synuclein[A53T] and TRAP1[T494A]; $\alpha$-Synuclein[A53T] and TRAP1[Y498G]; were assayed for mitochondrial membrane potential. No significant differences (1-way ANOVA) were noted. Left graph Control= empty vector+ TRAP1; right graph Control $=\alpha$-Synuclein[A53T]+ TRAP1. $n=6$ for + TRAP1, $n=3$ for TRAP1 mutants.

\subsubsection{Effect of TRAP1 mutation on total cellular ATP}

Finally, it was hypothesized that the impaired function of the different ETC complexes mainly observed for TRAP1[D158N] and [T494A]-expressing cells might reduce overall ATP level. Therefore, cells expressing the mutant TRAP1 proteins were compared for total cellular

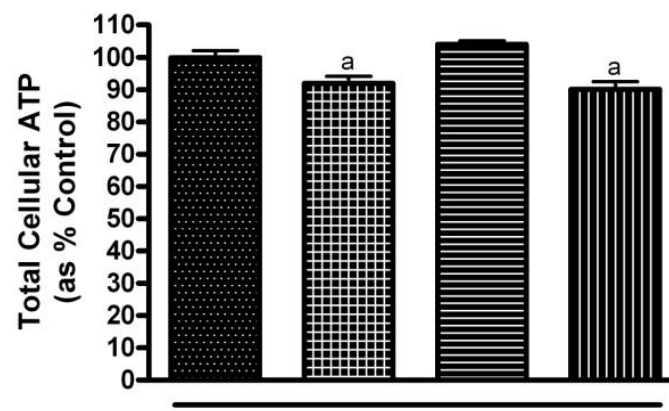

Empty Vector

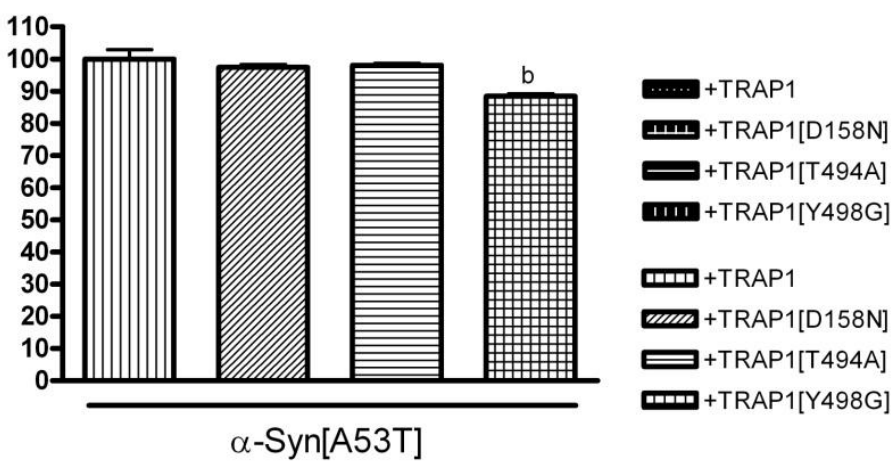

Fig. IV-46. Measurement of total cellular ATP for HEK293 cells expressing wt TRAP1 or TRAP1 mutants. TRAP1 overexpression was previously shown to not change total cellular ATP content. HEK293 cells transfected with: (left panel, left to right) empty vector and TRAP1 (Control); empty vector and TRAP1[D158N]; empty vector and TRAP1[T494A]; empty vector and TRAP1[Y498G]; (right panel, left to right): $\alpha$-Synuclein[A53T] and TRAP1 (Control); $\alpha$-Synuclein[A53T] and TRAP1[D158N]; $\alpha$-Synuclein[A53T] and TRAP1[T494A]; $\alpha$-Synuclein[A53T] and TRAP1[Y498G]; were assayed for total cellular ATP content. Significant differences (1-way ANOVA) observed: a: $\mathrm{p}<0.01$ vs. empty vector+ TRAP1; b: $\mathrm{p}<0.001$ vs. empty vector+ TRAP1[T494A]). In addition, $\alpha$-Synuclein[A53T]+ [Y498G] was significantly different than both other mutants $(\mathrm{p}<0.01$ for both $)$. Left graph Control= empty vector + TRAP1; right graph Control= $\alpha$ Synuclein[A53T]+ TRAP1. $n=6$ +TRAP1, $n=3$ for TRAP1 mutants. 
ATP content vs. wt TRAP1 expressing cells (Fig. IV-46). Interestingly, there was a reduction in ATP content seen with TRAP1[Y498G] when expressed alone or with $\alpha$-Synuclein[A53T]. The reason for the selective decrease in ATP under these conditions with [Y498G] is unclear, given that ETC complex production was not affected by this mutant. For TRAP1[D158N], the observed overall ATP deficit might result from the ETC Complex deficits previously noted.

\subsubsection{Effect of TRAP1 mutation on total mitochondrial protein}

As observed in previous experiments, certain TRAP1 mutations seem to cause an alteration in Complex I ATP production. This effect might be due to differing amount of mitochondria within the cells rather than being caused by a functional deficit of the ETC complexes. Thus, cell lysates were analyzed for abundance of the mitochondrial proteins VDAC1 and COX4 (Fig. IV-47). No changes in VDAC1 or COX4 protein levels were observed in cell lysates expressing either wt TRAP1 or mutant variants TRAP1, regardless if there was co-expression of $\alpha$-Synuclein[A53T] or not. This data thus indicates that mutant TRAP1 expression does not alter the overall mitochondrial content. This data therefore argues in favour of a functional ETC Complex deficit, rather than a ATP deficit resulting from amount of mitochondrial/ETC components in the cell. 

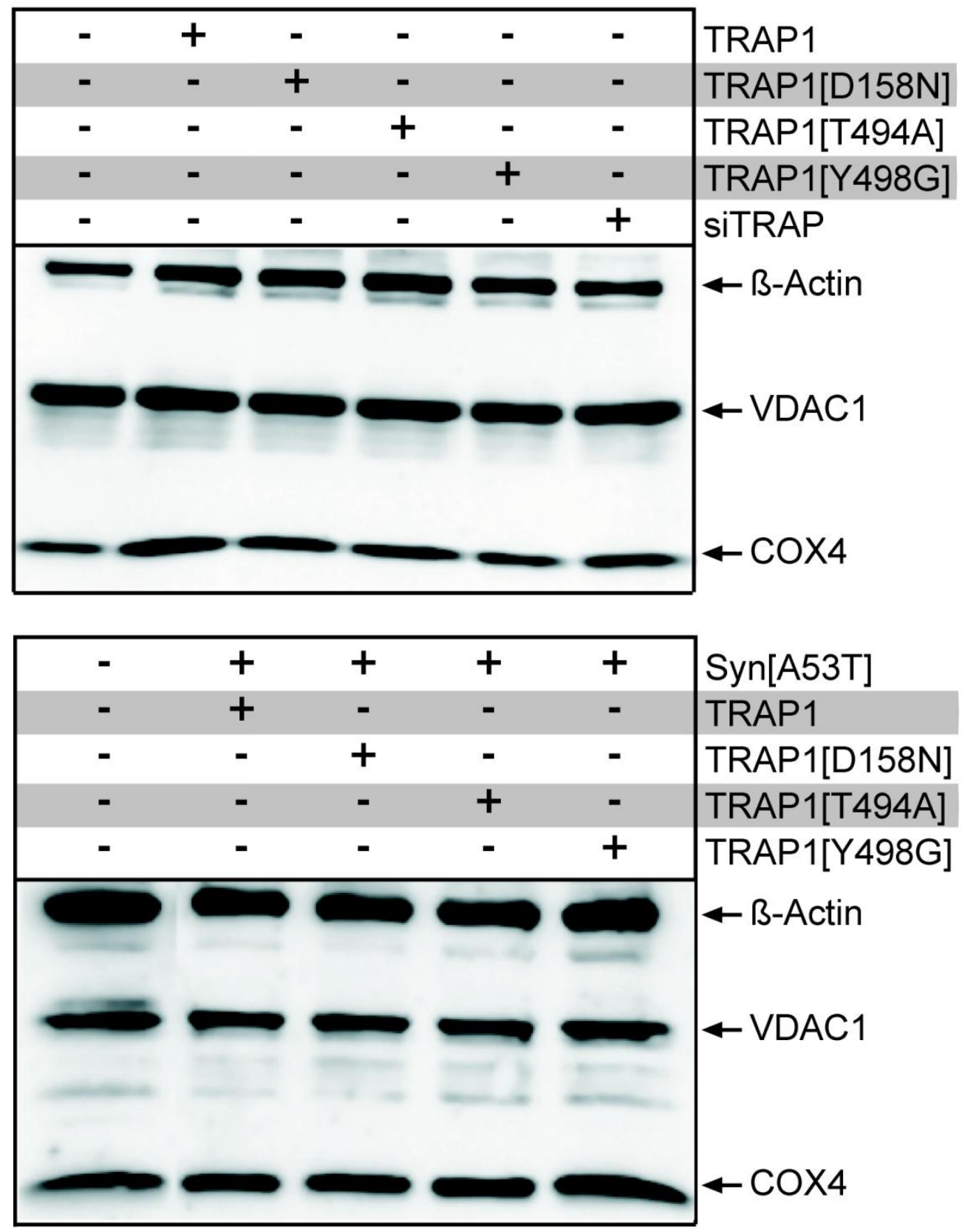

Fig. IV-47. Mitochondrial protein VDAC1 and COX4 expression in HEK293 cells expressing TRAP1 mutants $\pm \alpha$-Synuclein[A53T]. After transfection with indicated expression constructs, HEK293 lysates were used for Western blot analysis and probed with VDAC1 $(31 \mathrm{kDa})$, COX4 $(15 \mathrm{kDa})$ and B-Actin $(50 \mathrm{kDa}$ protein loading control) specific antibodies. Top panel shows expression of actin, VDAC and COX4 for cells expressing TRAP1 alone and bottom panel shows expression of the same for cells expressing TRAP1 mutants and $\alpha$ Synuclein[A53T]. Top panel lanes from left to right: 1) Control (empty vector+ scramble siRNA), 2) Empty Vector+ TRAP1, 3) Empty Vector+ TRAP1[D158N], 4) Empty Vector+ TRAP1[T494A], 5) Empty Vector+ TRAP1[Y498G], 6) Empty Vector+ siTRAP1. Bottom panel lanes from left to right: 1) Control (empty vector+ scramble siRNA), 2) $\alpha$-Synuclein[A53T] + TRAP1, 3) $\alpha$-Synuclein[A53T] + TRAP1[D158N], 4) $\alpha$ Synuclein[A53T]+ TRAP1[T494A], 5) $\alpha$-Synuclein[A53T]+ TRAP1[Y498G]. Representative blots after 3 experiments shown. 


\subsubsection{Modification of $\alpha-S y n u c l e i n[A 53 T$ ] Toxicity by TRAP1 in Rat Primary Cortical Neuron Culture}

\subsubsection{Overexpression of TRAP1 and $\alpha$-Synuclein[A53T] in primary cortical neurons}

In fly, overexpression of human TRAP1 was able to reduce $\alpha$-Synuclein[A53T]induced sensitivity to oxidative stress and loss of DA neurons. These findings concerning oxidative stress sensitivity were reproduced in HEK293 human cell culture. However, as HEK293 cells are not terminally differentiated cells and survival differences observed might reflect quiescence rather than cell death mechanisms. Experiments measuring cell survival after oxidative stress treatment must therefore be carefully interpreted. Thus, to additionally confirm that overexpression of human TRAP1 is able to rescue $\alpha$-Synuclein[A53T]-induced sensitivity to oxidative stress in a vertebrate neuron, terminally-differentiated cell model, rat primary neuron cultures were used for oxidative stress survival assays. Neurons are poorly transfected with classical transfection reagents, therefore lentiviruses were generated allowing efficient infection of these cells. First lentivirus-facilitated expression of TRAP1 or $\alpha$ Synuclein[A53T] after infection of cortical neurons was examined (Fig. IV-48). Western blot analysis of cell lysates after infection with respective viruses clearly indicate that infection of cortical neurons resulted in robust expression of the target protein.

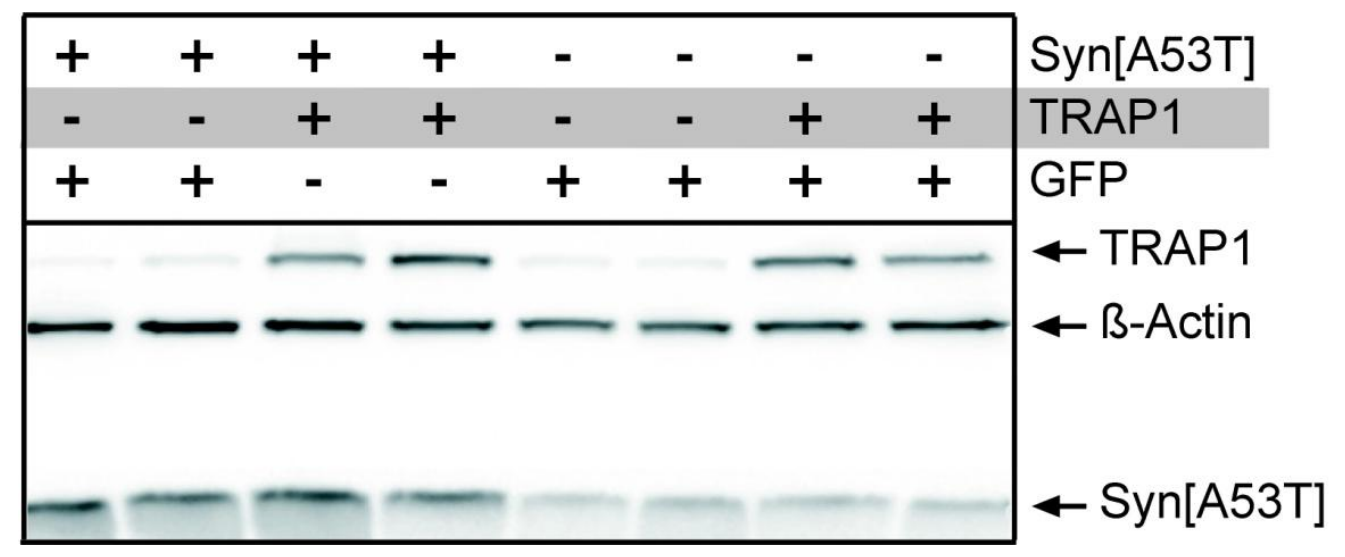

Fig. IV-48. Overexpression of TRAP1 and $\alpha$-Synuclein[A53T] in rat primary cortical neurons. After infection with indicated lentiviruses, rat cortical neuron lysates were used for Western blot analysis and probed with TRAP1 $(75 \mathrm{kDa}), \alpha$-Synuclein $(16 \mathrm{kDa})$ and $\beta$-Actin $(50 \mathrm{kDa}$ protein loading control) specific antibodies. Samples were collected 6 days after virus infection. Lanes left to right: 1,2) $\alpha$-Synuclein[A53T] +GFP control virus; 3,4) $\alpha$-Synuclein[A53T] +TRAP1 viruses; 5,6) GFP control virus alone and 7,8) TRAP1+ GFP control virus. The $\alpha$-Synuclein specific antibody used in this analysis cross reacts with rat $\alpha$-Synuclein, as seen by the 


\section{RESULTS}

weak signal in lysates from cells without viral induced expression of human $\alpha$-Synuclein[A53T]. Representative blot after 3 experiments.

\subsubsection{Confirmation of neuronal phenotype and viral infection in rat primary cortical}

\section{culture}

Cortical neuron preparations contain not only neurons but also other cells found within the brain, including astrocytes, oligodendrocytes and glia. Thus, in order to determine the percentage of neurons within the cultures, neuron preparations infected with a lentivirus resulting GFP expression were analyzed for co-localization of the neuronal marker NeuN with GFP signal (Fig. IV-49). More than $70 \%$ of the cells stained with the neuronal marker. $90 \%$ of the NeuN positive cells (neurons) also showed GFP expression, indicating a very high infection efficacy. Thus, specific survival of neurons can therefore be assessed using the rat primary cortical preparation.

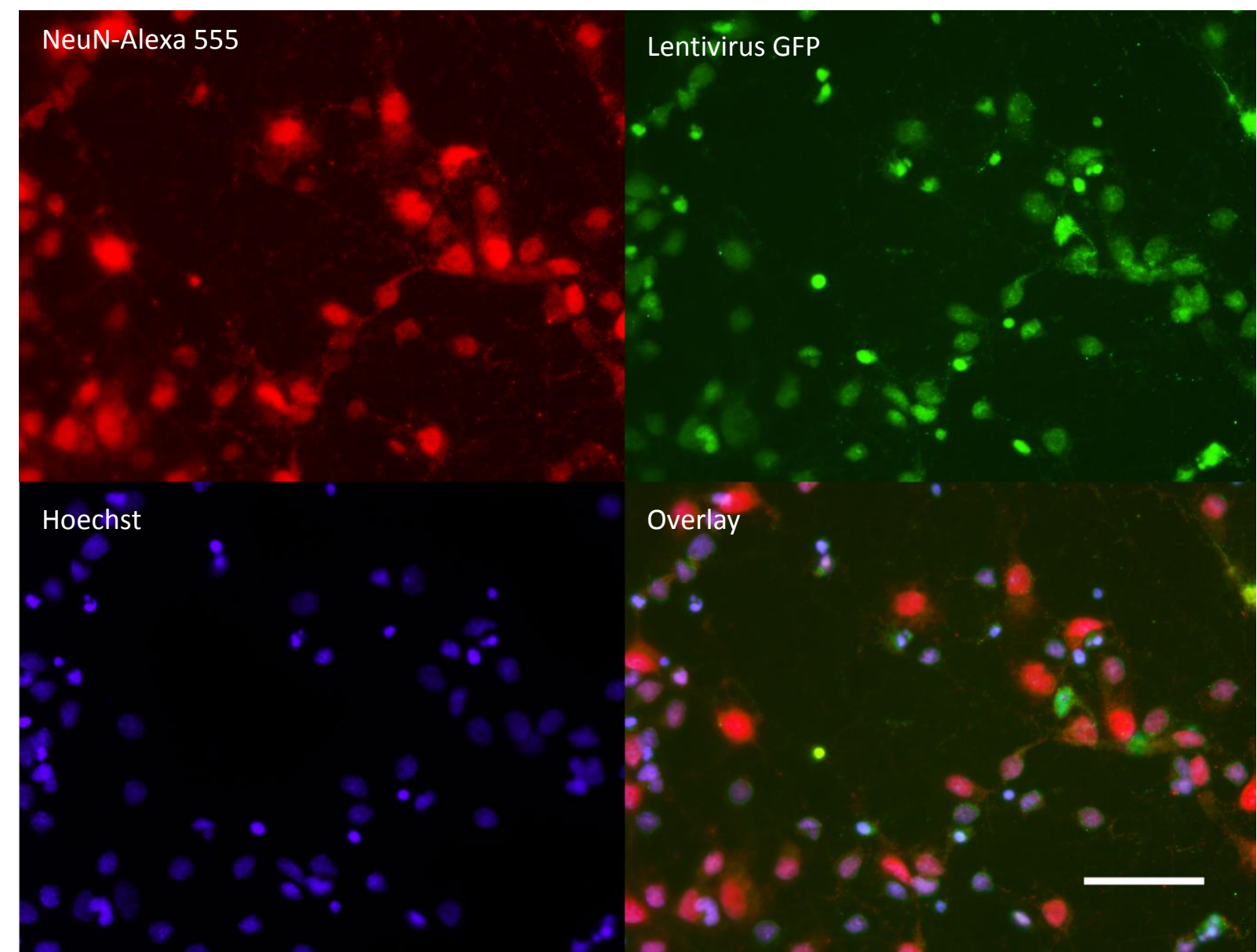

Fig. IV-49. Virally infected rat primary cortical culture stained for NeuN. A high percentage of cells show GFP expression indicating infection with lentivirus expressing $\alpha$-Synuclein[A53T] and the neuronal marker NeuN (red). Blue $=$ Hoechst (nuclear dye). Micrographs taken on Leica fluorescent microscope. Scale bar=43 um. 


\subsubsection{Rotenone treatment of primary cortical neurons}

As seen in HEK cells, TRAP1 might also protect primary cortical neurons from $\alpha$ Synuclein[A53T] induced toxicity. In analogy to other cell types, cortical neurons did not displayed robust toxicity upon $\alpha$-Synuclein[A53T] alone. Therefore, cultured cells were exposed to low doses of the mitochondrial complex I inhibitor rotenone. Compared to GFPvirus infected cells (control), co-expression of $\alpha$-Synuclein[A53T] resulted in an enhanced sensitivity to rotenone (Fig. IV-50). In agreement with fly and HEK293 data, coincident overexpression of TRAP1 was able to restore survival to control values. Interestingly, expression of TRAP1 alone enhanced survival beyond that of control cells, indicative of a protective effect of TRAP1 on neurons independent of effects on toxicity.

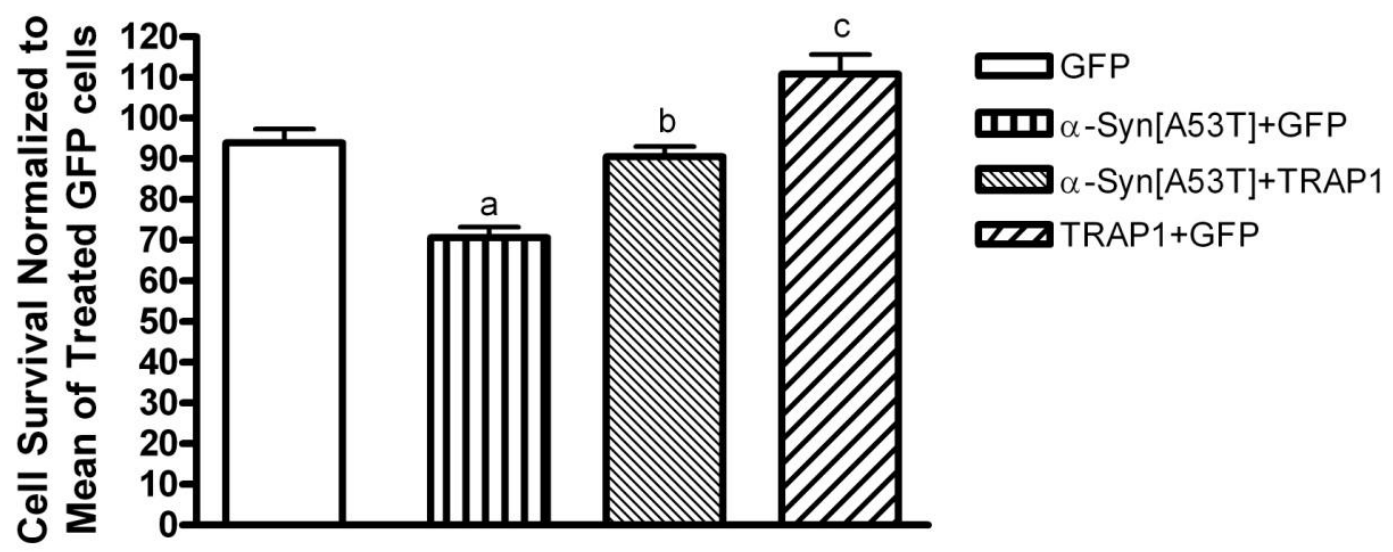

Fig. IV-50. Survival of primary cortical neurons following rotenone treatment. Primary rat cortical neurons were infected with: (left to right): GFP; $\alpha$-Synuclein[A53T] and GFP; $\alpha$-Synuclein[A53T] and TRAP1 and TRAP1 and GFP-expressing viruses; and treated with rotenone $(1 \mu \mathrm{M})$ for 16 hours. Significant differences (1way ANOVA) observed: a: $p<0.001$ vs. GFP; $b: p<0.001$ vs. $\alpha$-Synuclein[A53T]+ GFP; $c: p<0.05$ vs. GFP). $\mathrm{n}=3$. 


\subsubsection{Data Summary from Investigation into TRAP1 modification of $\alpha$-Synuclein[A53T] Toxicity}

D.melanogaster:

- Decrease in TRAP1 enhanced $\alpha$-Synuclein[A53T] toxicity in flies

○ Enhancement to:

- Loss of brain DA

- Sensitivity to hydrogen peroxide and paraquat

- Loss of climbing ability

- Loss of TH-neurons in PPL1 and PPM1/2 cluster

- Overexpression of human TRAP1 was able to rescue the above noted phenotypes except for PPL1 cluster TH neuron loss

Human HEK293 cells:

- $\quad \alpha$-Synuclein[A53T], TRAP1 and siTRAP1 expressed in HEK293 cells

- $\quad \alpha$-Synuclein[A53T] fraction localized to mitochondria

○ Co-immune localization using confocal optical slicing for Mitotracker Orange and $\alpha$-Synuclein[A53T]

- Cell fractionation with $\alpha$-Synuclein[A53T] in the mitochondrial enriched fraction in Western blot

- Expression of $\alpha$-Synuclein[A53T]+ siTRAP1 sensitizes cells to oxidative stress (treatment with hydrogen peroxide or rotenone)

○ TRAP1 overexpression provides rescue

- Complex I ATP production is reduced in cells with $\alpha$-Synuclein[A53T] expression

○ Combination of siTRAP1 enhances deficit

- TRAP1 overexpression provides rescue

- No differences observed for Complexes II, III and IV

- Total cellular ATP content unchanged with $\alpha$-Synuclein[A53T] \pm TRAP1

- Mitochondrial membrane potential (JC-1 measurement) reduced with $\alpha$ Synuclein[A53T] and siTRAP1 transfection

- $\quad$ Protein-protein interaction between TRAP1 and $\alpha$-Synuclein[A53T] found using IP

- Oxidative stress enhances tyrosine phosphorylation of wt TRAP1, with coexpression of $\alpha$-Synuclein[A53T] enhancing of phospho-threonine, tyrosine and serine

- $\quad$ TRAP1 [D158N] (putative ATPase dead) shows reduced rescue effect of $\alpha$ Synuclein[A53T] -induced sensitivity to rotenone 16 hour treatment

- TRAP1 [D158N] and phosphorylation mutant [T494A] shows less effective rescue of $\alpha$-Synuclein[A53T] -induced Complex I defect

- $\quad$ TRAP1 [D158N] and [T494A] both interfere with Complex II ATP production in the presence of $\alpha$-Synuclein[A53T]

- $\quad$ TRAP1 [T494A] reduces Complex IV activity with $\alpha$-Synuclein[A53T] expression

- Total cellular ATP reduced with TRAP1[Y498G] with $\alpha$-Synuclein[A53T] expression

- $\quad$ No differences in mitochondrial membrane potential observed for TRAP1 vs. TRAP1 mutants and $\alpha$-Synuclein[A53T]

- $\quad$ TRAP1[D158N] mutant data indicates limited protein-protein interaction with $\alpha$-Synuclein[A53T]

Rat Primary Cortical Neurons:

- $\quad$ Lentiviral expression of $\alpha$-Synuclein[A53T] enhances sensitivity to rotenone

○ Overexpression of TRAP1 rescues $\alpha$-Synuclein[A53T]-induced sensitivity

- Expression of TRAP1 alone reduces sensitivity above that of Control 


\subsection{Secondary Screen for Mitochondrial Related Genes Modifying $\alpha$ - Synuclein[A53T] Toxicity}

Single genes analysis from candidate deficiencies identified in the primary screen was primary performed in an unbiased way. However, as first single genes candidates were linked to mitochondria and their function, a cross reference with screening data from a recently published mitochondrial function genetic screen seemed useful. Targeting genes relating to the mitochondria seemed a reasonable step, given that both of the first two genes identified in the screen as candidates (TRAPl and $k d n$ ) were mitochondrial-related, and at the same time, multiple studies linking $\alpha$-Synuclein to mitochondrial function had been recently published. Chen et al used genome-wide RNAi in Drosophila cell culture to identify genes affecting mitochondrial function, as assessed by alteration to CS activity. ${ }^{242} \mathrm{~A}$ total of 153 hits were identified. The Chen et al results were then cross referenced to genes found within the lethal, semi-lethal, 'low' and 'high' deficiency categories of the HPLC screen. Twenty two genes identified by Chen and co-workers were located in deficiencies identified in HPLC screen to alter DA content in fly heads. Flies with RNAi-mediated gene silencing of these genes were then individually screened for alteration in brain DA content, longevity and sensitivity towards oxidative stress. Several other genes of noted in Chen et al screen thought to be of interest were also screened, although they could not be located in the above mentioned deficiency categories (e.g. PPI- involved in DA synaptic transmission ${ }^{243}{ }^{244}$; Suvarregulator of SUMO modification and as addition, several SUMO related genes ${ }^{245}$ ). Candidates which indeed reduced DA compared to controls were confirmed (for full list see Appendix Results Table 3). Genes that showed alteration to more than one endpoint are summarized in Table IV-2. In total, 17 additional candidate genes were identified as altering at least one of the end points relating to $\alpha$-Synuclein[A53T] toxicity (DA content, oxidative stress tests or longevity). In addition to genes connected to directly to mitochondria, multiple genes 
involved in protein processing and degradation were identified, and thus implicated as being important to mitochondrial function.

\begin{tabular}{|c|c|c|c|}
\hline Gene & Loss of DA & $\begin{array}{l}\text { Sensitivity to Oxidative } \\
\text { Stress }\left(\mathrm{H}_{2} \mathrm{O}_{2} \text { or Paraquat }\right. \\
\text { Treatment) } \\
\end{array}$ & Longevity Alteration \\
\hline Tiny Tim 50 (CG2713) & Enhanced & $\begin{array}{l}\text { Increased sensitivity to } \\
\mathrm{H}_{2} \mathrm{O}_{2}\end{array}$ & Reduced \\
\hline Bellweather (CG3612) & $\begin{array}{l}\text { Enhanced with both } \\
\text { drivers }\end{array}$ & $\begin{array}{l}\text { Increased sensitivity to } \\
\text { paraquat with elav }\end{array}$ & \\
\hline $\operatorname{Src42A}(\mathrm{CG} 7873)$ & Enhanced & $\begin{array}{l}\text { Increased sensitivity to } \\
\mathrm{H}_{2} \mathrm{O}_{2}\end{array}$ & \\
\hline CCT $\delta(\mathrm{CG} 8258)$ & Enhanced & $\begin{array}{l}\text { Increased sensitivity to } \\
\mathrm{H}_{2} \mathrm{O}_{2} \text { with elav }\end{array}$ & \\
\hline p70 S6k (CG10539) & Enhanced & $\begin{array}{l}\text { Overexpression enhances } \\
\text { sensitivity to } \mathrm{H}_{2} \mathrm{O}_{2}, \mathrm{RNAi} \\
\text { decreases; opposite for } \\
\text { paraquat }\end{array}$ & \\
\hline Thread (CG12284) & Enhanced & & Reduced \\
\hline Deflated (CG18176) & Enhanced & $\begin{array}{l}\text { Enhanced sensitivity to } \\
\mathrm{H}_{2} \mathrm{O}_{2}\end{array}$ & Enhanced \\
\hline Barren (CG10726) & Enhanced & $\begin{array}{l}\text { Increased sensitivity to } \\
\mathrm{H}_{2} \mathrm{O}_{2}\end{array}$ & \\
\hline $\begin{array}{l}\text { Phosphoglycerate mutase } \\
\text { (CG5165) }\end{array}$ & Enhanced & $\begin{array}{l}\text { Increased sensitivity to } \\
\text { paraquat }\end{array}$ & \\
\hline
\end{tabular}




\section{Discussion.}

\subsection{Brief Summary of Key Findings}

In the present study, a genetic screen in D. melanogaster was performed to identify new modifiers of $\alpha$-Synuclein[A53T] toxicity. In the primary screen, flies expressing $\alpha-$ Synuclein[A53T] in aminergic neurons were crossed with fly lines derived from the 'Bloomington Deficiency Kit', carrying a certain chromosomal deletion. In the F1 generation, flies with $\alpha$-Synuclein[A53T] expression and a respective deletion were analyzed with regard to reduction in brain DA with time. Twenty four deficiencies were identified to enhance $\alpha$ Synuclein[A53T]-induced DA loss. The deficiency resulting in the greatest loss of DA was selected for further investigation in order to isolate the single gene responsible for this phenotype. Reduced expression of the mitochondrial chaperone TRAP1 was pinpointed as enhancing $\alpha$-Synuclein[A53T] toxicity. TRAP1 reduction in $\alpha$-Synuclein[A53T]-expressing flies not only enhanced DA loss, but also enhanced loss of DA neurons and resulted in impaired locomotion. In addition to these age-dependent effects, reduction in TRAP1 sensitized flies towards oxidative stress, an effect more pronounced when $\alpha$-Synuclein[A53T] was co-expressed. All phenotypes could however be rescued by overexpression of human TRAP1.

Cell culture experiments underlined the protective effects of TRAP1. First, in HEK293 cells, $\alpha$-Synuclein[A53T] caused a decrease in Complex I activity and when in combination with siTRAP1, reduced the mitochondrial membrane potential under resting conditions. In these cases, TRAP1 overexpression also restored the observed defects to control values. Analysis of mutations in TRAP1 suggested an importance of both its ATPase domain and phosphorylation state to its protective functions. Finally, rat primary neurons showed enhanced sensitivity to oxidative stress in the presence of $\alpha$-Synuclein[A53T] expression, while coincident overexpression of TRAP1 provided a rescue effect. This data cumulatively 


\section{DISCUSSION}

argues in favor of $\alpha$ - Synuclein[A53T] exerting its toxicity, at least partially, in mitochondria. In agreement, $\alpha$-Synuclein[A53T] was found to co-localize with mitochondrial markers and a direct protein-protein interaction between TRAP1 and $\alpha$-Synuclein[A53T] was observed in IP experiments.

Thus, this study describes the mitochondrial chaperone protein TRAP1 as a novel modifier of $\alpha$-Synuclein[A53T] toxicity in fly, human cell culture and rat primary neurons. A key importance of the mitochondria to both $\alpha$-Synuclein toxicity and toxicity suppression are thereby implicated.

\subsection{Genetic Screen Results}

5.2.1. Limitations of the primary and secondary genetic screens for modifiers of $\alpha$ - Synuclein[A53T] neurotoxicity

Before comparing results of the primary and secondary screens to previously published screens and data relating to $\alpha$-Synuclein, several important topics must be first discussed in order to correctly assess the data.

First, obvious limitations of the primary screen should be outlined. Genetic screening utilized an endpoint of alteration in fly brain DA, measured using HPLC. Although loss of brain DA is a relatively subtle end point, as it occurs on a gradient, this endpoint is of immediate relevance to PD pathogenesis. However, the requirement of HPLC analysis made this approach not practical for a genome-wide approach utilizing single gene mutations or single gene silencing via RNAi. Depending on the library used, such a screening approach would require at least 8,000 (roughly the number of all fly genes displaying a human homolog) crosses and subsequent DA analysis. Use of the 'Bloomington Deficiency Kit' limited the number of fly crosses to 270 . However, the chromosomal deletions can affect several genes, up to more than hundred. Indeed, the total number of genes affected by deficiencies identified to significantly reduce DA (and thus considered for further analysis) 


\section{DISCUSSION}

was well over 1000. The identification of the single gene, responsible for the decrease in DA, requires sub-screening of the genes deleted by a given deficiency. ${ }^{246,247}$

Although using deficiencies allows a genome wide analysis, not all modifiers of $\alpha$ Synuclein[A53T] induced toxicity will be identified utilizing a deficiency screen. For example, a deficiency could contain genes with opposing effect, with one gene acting to increase DA, while the other acting to decrease, with the summed effects resulting in no change in DA. Alternatively, multiple genes might act in the same direction could be located in the same loci, resulting in significant increase or decrease in DA. However, loss of the individual gene might not affect DA content to any noticeable effect. This limits the conclusions that can be drawn from any one deficiency result, before individual gene subscreening has been carried out. In addition, the used screening paradigm is based on the assumption that a $50 \%$ reduction of a given modifier is enough to render strong effects on DA. This of course limits false positive interactions, but on the other hand might miss important factors. However, despite these limitations of the chosen screening approach, the major advantage of the primary screen remains that the end point is one clearly related to PD pathogenesis. Thus, modifiers identified through this process should likely be of direct relevance to the age-dependent time course of $\alpha$-Synuclein[A53T] toxicity.

In order to find additional specific targets beyond TRAP1, and avoid laborious and impractical gene by gene HPLC screening, 'low' results from the primary screen were cross referenced with data from a mitochondrial screen. That is, a hypothesis- based approach was utilized. Recent papers had demonstrated the mitochondria as an additional pathway for $\alpha$ Synuclein toxicity, beyond that of aggregation, ER-Golgi and vesicular dynamics. $\alpha$ Synuclein and $\alpha$-Synuclein[A53T] were shown able to enter the mitochondria and entry was associated with derangement of function. ${ }^{46,173,174,180}$ Thus, of interest were factors relating to the mitochondria, factors which might be connected to the loss of DA endpoint of the primary 


\section{DISCUSSION}

screen. Loss of DA might be connected to mitochondrial dysfunction via two avenues. Firstly, neuron dysfunction may occur due to a mitochondrial precipitated energy crisis. Secondly, DA neuron loss might result from mitochondrial- dependent apoptosis. The importance of mitochondria was further suggested by both the TRAP1 outcome from the primary screen and the finding that the combination of citrate synthase (CS) activity and $\alpha$-Synuclein[A53T] expression resulted in lethality. The Chen et al screen was the only screen found dealing with Drosophila mitochondria function and thus the genes highlighted provided a useful staging ground for a secondary screen. ${ }^{242}$ In particular, the genes in the Chen et al screen were identified using overall CS activity as end point for altered mitochondrial function. This choice of end point was rationalized on the basis that CS activity has been previously used as a marker indicative of oxidative capacity and has been shown to correlate mitochondrial DNA content in muscle. ${ }^{248,249}$ Thus, the screen seemed a useful tool for generating modifiers related to $\alpha$-Synuclein-associated mitochondrial function. Of course, the primary screen data could also be cross referenced with other screening data, to generate additional genes for HPLC screening.

Several additional points are worth mentioning before moving onto data comparison. The screen utilized an aging-dependent model of $\alpha$-Synuclein[A53T] toxicity, as assessed by loss of brain DA. This description relates three critical factors that must be considered when interpreting screen results in comparison to data previously published. The first being that the toxicity assessed was aging-dependent, and was not an effect observable during the initial period of overexpression. ${ }^{135}$ Thus, the screen is more closely applicable to the native course of PD pathogenesis. The second point refers to how toxicity was enhanced. An exogenous toxin was not applied and then assessed for alteration, but rather the native course of $\alpha$-Synuclein toxicity was effected on the basis of a genetic alteration to the fly. This implies a direct genetic interaction, rather than a reaction secondary to a toxic mediator. Lastly, $\alpha$ - 


\section{DISCUSSION}

Synuclein[A53T] was used. Multiple papers have demonstrated that mechanism of toxicity may be dependent on the $\alpha$-Synuclein variant. ${ }^{132,169,250-254}$ For example, a yeast screen that looked for $\alpha$-Synuclein suppressors found that the top five identified suppressors of wt $\alpha$ Synuclein were ineffective in suppressing toxicity resulting from [A30P] or [A53T] Synuclein. ${ }^{250}$ Thus, it would not be surprising that genetic modifiers found for $\alpha$-Synuclein wt or $\alpha$-Synuclein[A30P] might be different than those generated when investigating $\alpha$ Synuclein[A53T]. All of these points are important to keep in mind when evaluating the results of the present screen in comparison to previously published screens involving $\alpha-$ Synuclein.

\subsubsection{Comparison of candidates to previously identified $\alpha$-Synuclein modifiers and genetic screens}

Several modifiers of $\alpha$-Synuclein have been identified through hypothesis-based testing. In particular, the molecular chaperone protein HSP70 has been found associated with $\alpha$-Synuclein in the LB. Overexpression of human HSP70 was reported by Auluck et al in 2002 to reduce DA neuronal loss secondary to ddc driver expression of either wt $\alpha$-Synuclein or Synuclein [A53T] in Drosophila. ${ }^{142,197}$ Expression knock down of a major endogenouslyexpressed chaperone with homology to human HSPs, Hsc4, enhanced $\alpha$-Synuclein-induced DA neuronal loss. ${ }^{142}$ Hsc4 is located in deficiency BL1534 (Tp(3;Y)ry506-85C), a large deletion containing over 300 genes. For this deficiency cross, no enhancement of DA loss was noted. It may be that the enhancer genes were also deleted, thus acting to normalize DA content. Alternatively, the loss of $50 \%$ of $\mathrm{Hsc} 4$ gene product using a deficiency line may not have been sufficient to enhance the toxicity of $\alpha$-Synuclein (vs. near $100 \%$ elimination using a dominant-negative mutation). HSP70 was not a gene analyzed in the secondary mitochondrial-related screen. Worth noting however, is that deficiency BL3128 (Df(3R)MKx1) contains 6 isoforms of endogenous HSP70 (all without homology to human HSPs). This 


\section{DISCUSSION}

deficiency cross resulted in a large decrease of DA at 4 weeks $(54.85 \%)$, but it did not fall within the 'low' category to be further pursued. Thus, while it is possible that the native HSP70s also play a role in modifying toxicity of $\alpha$-Synuclein, HSP70 suppression of $\alpha$ Synuclein was not re-confirmed. In addition to HSP70, two other proteins: Sirtuin2, a histone deacetylase with multiple cell regulatory functions, and $\mathrm{Cu} / \mathrm{Zn} \mathrm{SOD}$, an antioxidant protein, have also been identified as modifiers of $\alpha$-Synuclein toxicity in the fly using hypothesisbased testing. ${ }^{201} 255$ Neither of these genes were found in deficiency crosses categorized as either 'low', 'high,' 'semi-lethal' or 'lethal.' As noted above, there are multiple reasons why a specific gene may or may not be located in a deficiency cross categorized as 'low'.

Several genetic screens, completed in either yeast or worm, have previously searched for novel modifiers of $\alpha$-Synuclein toxicity. Both worm screens measured the effect of RNAi gene knockdown on modification to wt $\alpha$-Synuclein aggregation in the cytoplasm. ${ }^{107,256}$ The yeast screens searched for modification of $\alpha$-Synuclein toxicity using an end point of cell viability. ${ }^{60,250,257}$ Alongside several hits relating to stress response and ubiquitin-mediated protein degradation, the screens generated a preponderance of genes related to lipid metabolism and vesicle-mediated transport. Although the present screen was oriented toward mitochondrial function, it also highlighted several genes related to the protein degradation (thread, CCT $\delta$, Uevla, smt3, Aos1, Parkin) or autophagy (P70 S6k) as either reducing DA content or increasing sensitivity to oxidative stress. This emphasizes that protein degradation pathways are key to the pathology of both wt and mutant $\alpha$-Synuclein and that aggregation can be linked to mitochondrial function. Importantly, as mentioned in the Introduction, the van Ham et al worm screen identified R151.7 as a candidate worm gene, with putative expression knockdown resulting in premature $\alpha$-Synuclein aggregation. ${ }^{107} R 151.7$ is a worm mitochondrial chaperone, homologue to Drosophila and human TRAP1. ${ }^{223}$ This data acts as an external confirmation that TRAP1 is able to modulate the toxicity of $\alpha$-Synuclein. It also 
suggests that TRAP1's protective mechanism is additionally linked to alteration of cytosolic aggregation, an end point not examined in the present study.

In summary, these data together suggest that mechanisms of $\alpha$-Synuclein toxicity directly related to ER-Golgi function and lipid metabolism seem largely independent of mitochondrial toxicity, at least in terms of genetic modifiers to date identified. At the same time, given the findings of UPS-associated proteins found in all genetic screens including the present study, a common importance of the UPS system to $\alpha$-Synuclein toxicity is implicated.

\subsubsection{Secondary mitochondrial-related screen: discussion of targets generated}

Several gene candidates identified in the mitochondrial-associated secondary screen are worthy of further mention. Genes directly associated with mitochondrial function were highlighted as modifiers: Tiny Tim $50^{258}$ and bellweather. In addition, several genes involved in central control of energy homeostasis, that being carbohydrate metabolism (glycolysis enzyme phosphoglucomutase, ER carbohydrate modifier alpha man II and Krebs Cycle CS) also modified $\alpha$-Synuclein[A53T] toxicity. In this study, cell culture data indicated that $\alpha$ Synuclein is able to inhibit Complex I activity, although overall ATP levels remained unchanged. The lack of change to overall ATP suggests a hypothesis whereby other pathways involved in ATP production, that being glycolysis, could be upregulated to supply ATP sufficient to the cell's needs under baseline conditions. Thus, decrease in expression of genes associated with these pathways would interfere with such compensation. Such a hypothesis is supported by proteome data from $\alpha$-Synuclein-expressing Drosophila. In comparison to younger flies, old flies show upregulation of a wide range of genes involved in energy homeostasis, in particular many genes of the glycolysis and Krebs Cycle such as phosphofructokinase, succinyl CoA synthetase and the ETC, including ATP synthase. ${ }^{259-261}$ Pennington et al investigated mitochondrial proteome changes due to $\alpha$-Synuclein expression in human SH-SY5Y cells and recorded similar results. ${ }^{251}$ Similarly, several genes involved in 


\section{DISCUSSION}

carbohydrate metabolism were found to suppress $\alpha$-Synuclein aggregation in the van Ham et

al C. elegens $\alpha$-Synuclein screen. ${ }^{107}$ Thus, cellular response to $\alpha$-Synuclein involves core, evolutionary conserved pathways of energy homeostasis in the face of alteration to ETC components and efficiency.

In addition to the earlier mentioned proteins linked to protein degradation and ubiquitin modification, proteins associated with SUMO (Suvar, Aos1, lwr) modification, were found to modify $\alpha$-Synuclein toxicity. ${ }^{262} 263264$ Thus, it seems possible that SUMOylation of $\alpha$-Synuclein may have functional consequences, especially in context of response to oxidative stress. Further investigation seems warranted. Finally, putative p70 S6k overexpression reduced $\alpha$-Synuclein toxicity while targeted-RNAi line usage decreased DA loss and sensitivity to hydrogen peroxide. $p 70 S 6 k$ is involved in multiple Rheb-target-of rapamycin (mTOR) signaling pathways, including apoptosis and autophagy. ${ }^{231,265,266}$ That opposite results were observed when using putative overexpressor vs. an RNAi line is strongly suggestive of a significant role for $p 70 S 6 k$ in mediating $\alpha$-Synuclein toxicity. Whether that role is one involves in autophagy or apoptosis, or both, and what the relative contribution of each process is to overall cell survival in the context of $\alpha$-Synuclein, remains to be determined.

\subsection{TRAP1 modification of $\alpha$ - Synuclein[A53T] toxicity in Drosophila, HEK293 cells and primary neurons: conclusions, limitations and unanswered questions}

In this study, expression of $\alpha$-Synuclein[A53T] in Drosophila resulted in agingdependent phenotype of: brain DA loss, loss of TH neurons and a climbing-ability deficit. Fly sensitivity to exogenously applied oxidative stress was also enhanced. Loss of TRAP1 was shown to enhance detrimental effects of $\alpha$-Synuclein[A53T], while overexpression of hTRAP1 rescued $\alpha$-Synuclein[A53T] induced toxicity in all tested paradigms. 


\section{DISCUSSION}

The finding that $\alpha$-Synuclein[A53T] expression in aminergic neurons (ddc-GAL4) causes a decline in amount of $\mathrm{TH}$ neurons is in agreement with previously published Drosophila models of PD. ${ }^{135,199,201,210,267,268}$ In particular, TH neuron loss has been consistently reported in certain clusters or in overall TH number. ${ }^{135,142,197,201,210,222,255}$ The reason for the selective loss of DA neurons to PPL and DM is unclear, as little is known about differences in biochemistry between the Drosophila DA neurons. Differential susceptibility of DA neurons to death is seen in humans, and thus it is also plausible that a similar phenomenon could function in Drosophila. In either case, the data indicates that TRAP1 is able to rescue $\alpha$-Synuclein-induced toxicity in the PPM cluster. For this reason, this cluster was chosen for full analysis.

An additional aspect of TRAP1 function previously investigated is that of its antiapoptotic effects. As mentioned, in tumor cells, levels of TRAP1 are inversely correlated to levels of apoptosis. ${ }^{104,230,269}$ In multiple cell systems, high levels of TRAP1 appears able to reduce release of key factors involved in apoptosis, including Apoptosis Inducing Factor, Caspase-3 and Cytochrome c. ${ }^{103,105,229,230}$ Direct mechanisms by which TRAP1 might inhibit apoptosis were not examined in this study. However, given that overexpression of TRAP1 in both HEK293 and rat primary neuron culture was able to enhance cell survival after rotenone treatment similar to previous studies, it can be hypothesized that similar anti-apoptotic mechanisms might in part be responsible for TRAP1 rescue of $\alpha$-Synuclein[A53T] toxicity. PD-associated neuronal death has been shown to involve apoptotic cell death. ${ }^{270-274}$ Thus, in addition to effects enhancing mitochondrial health and energy production, TRAP1 overexpression in Drosophila DA neurons most likely is directly anti-apoptotic. Staining fly DA neurons for markers of apoptosis would be necessary in order to substantiate this hypothesis. 


\section{DISCUSSION}

Alteration to TRAP1 expression levels also had an influence on Drosophila susceptibility to oxidative stress treatment in either presence/absence of $\alpha$-Synuclein. Paraquat's toxicity results from an uncoupling of oxidative phosphorylation, as it accepts protons from donors such as NADPH, normally used by Complex I for ATP production. ${ }^{275,276}$ This seems to be the major point of action, as evident by the present analysis of $\alpha$ Synuclein[A53T] expression with regard to its effect on ETC in HEK cells (discussed below). In addition, paraquat can oxidize cytosolic Thioredoxin and activate JNK, followed by MAPK-mediated Caspase-3 dependent apoptosis and cell death. Thioredoxin regulates the stress response via redox-sensitive transcription factors and detoxification of ROS. Hydrogen peroxide is a more general inducer of oxidative stress, producing primarily hydroxyl free radicals via the Fenton reaction that can then oxidize cell proteins and damage mitochondria. Expression of $\alpha$-Synuclein[A53T] reduced survival upon paraquat treatment vs. control, however not after hydrogen peroxide treatment. This suggests that the toxicity engendered by paraquat is more closely related to $\alpha$-Synuclein[A53T]-toxic effects than hydrogen peroxide toxic effects. That is, presence of both $\alpha$-Synuclein[A53T] and paraquat may alter the same mitochondrial processes such as Complex I ATP production. TRAP1 overexpression enhanced survival of the $\alpha$-Synuclein[A53T] expressing flies under both stress paradigms. Reduction of endogenous TRAP1 in driver line flies reduced survival in both experiments, as would be expected from previous data indicating a decrease in TRAP1 to sensitize cells to oxidative stress. ${ }^{229,}{ }^{237}$ However, both previous and the present in vitro studies demonstrated that in different human cell types, endogenous TRAP1 overexpression can decrease sensitivity to hypoxic ischemic and ROS stress. In addition, TRAP1 has also been shown to reduce Caspase- 3 release. ${ }^{105,229,} 237$ This reduction in sensitivity was not observed in flies expressing human TRAP1. The reason for this is most likely that hTRAP1 is only expressed in few cells/neurons (ddc-GAL4 driver) and thus can exert its protective function only in 


\section{DISCUSSION}

these cells. The oxidative stress however is induced in all cells and thus an overall change in fly survival beyond control values was not observed.

Interesting is the case of hydrogen peroxide stress, where the greatest survival was found in $\alpha$-Synuclein[A53T]+ TRAP1 flies. In this situation, a possible explanation is that $\alpha-$ Synuclein[A53T] may take on a protective role via TRAP1 signaling. For example, if one accepts an assumption that TRAP1 directly binds $\alpha$-Synuclein[A53T] and somehow modifies it to be non-toxic (as suggested by the protein-protein IP data demonstrated in this study and putative TRAP1 chaperone function), then a small population of $\alpha$-Synuclein[A53T] might remain in the cell. Low concentration of $\alpha$-Synuclein might then prove to be protective. This is suggested by data showing a similar mechanism for wt Synuclein and HSP70. ${ }^{277}$ Further investigation into a possible protective effect $\alpha$-Synuclein in the fly brain is necessary to explain this finding.

The toxicity resulting from expression of $\alpha$-Synuclein[A53T] in both human cell and rat primary neuron culture agrees with previously published data. That being, expression of $\alpha-$ Synuclein can sensitize cells to oxidative stressors such as the Complex I inhibitor rotenone and oxidative stressor hydrogen peroxide, and can decrease Complex I activity. ${ }^{46,175-177,278,279}$ Of note was that the $\alpha$-Synuclein[A53T]-induced reduction in viability was enhanced in combination with siTRAP1. Co-expression of TRAP1 on the other hand, rescued $\alpha$ Synuclein[A53T] toxicity and cell viability was restored to control levels. This data is therefore supportive of a role for both $\alpha$-Synuclein[A53T] and TRAP1 ability to modulate Complex I behavior. Indeed, direct measurement of Complex I ATP production showed a decrease attributable to $\alpha$-Synuclein[A53T] expression, that was enhanced by siTRAP1 and restored by TRAP1 overexpression. ATP production by the other ETC complexes (II and IV) was neither reduced due to $\alpha$-Synuclein[A53T] expression nor did additional siTRAP1 have any effect. Although a clear reduction of ATP production of Complex I was observed in cells 


\section{DISCUSSION}

with $\alpha$-Synuclein[A53T] expression, total ATP levels were comparable to controls. It therefore might be possible that the cells expressing only $\alpha$-Synuclein[A53T] are able to initially compensate for this reduction, most likely via enhancement of glycolysis. ${ }^{280-282}$ However, cells with $\alpha$-Synuclein[A53T] expression in combination with siTRAP1 exhibited a decrease in overall ATP levels. Interestingly, these cells also displayed a significant reduction in mitochondrial membrane potential with and without oxidative stress treatment. Thus, $\alpha-$ Synuclein[A53T] expression with reduction of TRAP1 has a strong impact on mitochondrial function and seems to change the cellular energy metabolism.

It has been previously reported that decreased activity of oxidative phosphorylation reduces the mitochondrial membrane potential. Due to this drop in potential, the mitochondria then switch from ATP production to ATP consumption, in order to maintain the potential. ${ }^{283}$ Thus, when under the additional exogenous oxidative stress, the cells can neither defend against stress nor maintain the potential, leading to decreased survival, as was noted for $\alpha$ Synuclein[A53T]+ siTRAP1 cells.

As a final note, overexpression of TRAP1 alone was able to enhance ATP production from Complexes I and II. This finding has not been previously reported. It suggests a direct role for TRAP1 in modulating ETC components and an additional reason as to why TRAP1 overexpression is protective against oxidative stress. ${ }^{105,229,237}$ A simple explanation for the observed effects would be, that siTRAP1 reduces the amount of mitochondria, while TRAP1 overexpression preserves mitochondria, eventually leading to an increase in total number. However, the presented analysis of mitochondrial protein abundance indicates that overall mitochondrial load is not altered upon $\alpha$-Synuclein[A53T] expression. Similarly, siTRAP1 and TRAP1 overexpression did not alter overall mitochondrial protein abundance, indicating no depletion of mitochondria under these assay conditions. Thus, a direct effect of TRAP1 in alerting the function of the ETC components is implicated. 


\section{DISCUSSION}

The detrimental effects of $\alpha$-Synuclein[A53T] on Complex I suggest a mitochondrial localization of the protein. To prove $\alpha$-Synuclein[A53T] indeed can be found in mitochondria, fractionation experiments were performed. In these experiments, $\alpha$-Synuclein[A53T] could be found in the mitochondrial fraction. Moreover, immunofluorescence analysis revealed a colocalization of mitochondria with $\alpha$-Synuclein[A53T]. Finally, $\alpha$-Synuclein[A53T] was shown to co-precipitate with mitochondria-localized TRAP1. Moreover, mitochondrial localization of $\alpha$-Synuclein has been previously demonstrated in several other experimental systems, including cell culture, rat and PD patient brains. ${ }^{46,175-177,180}$ In addition, a direct association of $\alpha$-Synuclein with Complex I has been shown in PD brains and human fetal DA neurons. ${ }^{46}$ Together these data strongly suggest that at least a fraction of $\alpha$-Synuclein[A53T] in the cell can enter mitochondria and is involved/can alter organelle function.

The IP data indicating direct binding of $\alpha$-Synuclein[A53T]-TRAP1 may relate to TRAP1 chaperone function, with binding acting to inhibit $\alpha$-Synuclein aggregation or $\alpha$ Synuclein interaction with ETC components such as Complex I. Direct binding of TRAP1 (also known as HSP75) is suggested by the cytosolic interactions with $\alpha$-Synuclein of another heat shock chaperone, that being HSP70. That said, IP data is only the first step of delineating a protein- protein interaction. FRET for either in vitro or live cell measurements might be used for further investigation, although the ability of a fluorophore-labelled $\alpha$-Synuclein to enter the mitochondria is uncertain. Moreover, direct protein- protein interaction may not be strictly necessary for TRAP1 to have an effect on $\alpha$-Synuclein toxicity. That is, TRAP1's ability to lower ROS, preserve ATP levels, interfere with Caspase -3 activation, regulate the mitochondrial permeability pore and Cytochrome c release, may be the major ways in which it can counteract $\alpha$-Synuclein-induced toxicity. ${ }^{105,236,237,284103,229}$

Another point to mention is that a specificity of TRAP1 effect to $\alpha$-Synuclein is not a necessity for TRAP1 rescue. That is, while TRAP1 has been shown to act downstream of 


\section{DISCUSSION}

PINK1 in the context of PD, it may also be a more general modifier of mitochondrial behavior. Its pro-survival activity may therefore be activated in the context of multiple mitochondrial stressors, perhaps without specificity to $\alpha$-Synuclein. Confirmation of such a mechanism of action would however not alter the fact that it is indeed protective against $\alpha$ Synuclein toxicity. Further investigation is required to clarify the precise mechanics and functional implications of said interaction. However, based on the effects of both TRAP1 knockdown and TRAP1 overexpression on $\alpha$-Synuclein[A53T] toxicity observed in both fly and human/rat cell culture, it would be interesting to investigate TRAP1 KO in a mouse model of $\alpha$-Synuclein[A53T] expression. It can be hypothesized that TRAP1 KO would sensitize mice to $\alpha$-Synuclein[A53T] and perhaps reveal additional insights into the mitochondrial effects of the protein in an in vivo mammalian system.

The previously published features of TRAP1 function relating to survival after oxidative stress treatment were confirmed in the cell culture data presented. However, while oxidative stress combined with siTRAP1 reduced survival below control, similar to previous studies, overexpression in HEK293 of TRAP1 did not increase cell survival above control. However in primary rat cortical culture, expression of TRAP1 alone enhanced survival of cells after rotenone treatment. The reason for this difference in efficacy is unclear. As mentioned, TRAP1 overexpression in a variety of cell types, including astrocytes, has been shown protective against oxidative stress. One could speculate that HEK293 cells are in general less sensitive to oxidative stress, meaning that they constitutively express proteins such as TRAP1 to a higher level. This is suggested by the decreased quantity of rotenone needed to cause cell death in cortical culture vs. HEK293. Thus, additional TRAP1 expression would not further enhance survival. However, in primary neuron culture, endogenous TRAP1 expression may be lower and thus overexpression of TRAP1 provides additional protection. 


\section{DISCUSSION}

The fact that TRAP1 is reported to have chaperone function and is here shown to coprecipitate with $\alpha$-Synuclein[A53T] suggests a scenario in which TRAP1 prevents misfolded $\alpha$-Synuclein to exert detrimental effects in mitochondria. To analyze the TRAP1- $\alpha$-Synuclein interaction in more detail, several TRAP1 mutants were generated. In particular, the ATPase domain of TRAP1 has been implicated as important for both import into the mitochondria and for chaperone folding. ${ }^{285}$ ATP hydrolysis by chaperones is generally considered to be important for chaperone substrate release. Indeed, ATP/ADP cycling of chaperones implies substrate binding. As suggested by other HSP90 proteins, there may be an additional role for co-chaperones in TRAP1 regulation, which may also interact via the ATPase domain. ${ }^{286}$ Thus, abolishing ATP binding should interfere with TRAP1 endogenous function and should impact the ATPase domain in general. On the basis of sequence to homology to mutant ATPase dead yeast $\mathrm{HSP}^{241}$, the D158N-mutation was introduced to interfere with ATP binding domain of TRAP1. Of course, definitive structural analysis and biochemical proof of ATP binding deficit were not provided in this study and thus this provides a topic for an additional study. Thus, attribution to ATPase domain functional alteration as the reason for the functional effects of [D158N] mutation effect can only be regarded as tentative at present. That said, the TRAP1[D158N] will be referred to a TRAP1 variant with an altered function of the ATPase domain.

In addition, as mentioned in the Introduction, it was previously reported that TRAP1 acts downstream of PINK1. Pridgeon et al demonstrated that the kinase PINK1 phosphorylates TRAP1. Moreover, this phosphorylation is a prerequisite for mediation of PINK1's protective effects against oxidative stress and apoptosis. ${ }^{103}$ Thus, the presented data links PINK1 via TRAP1 to $\alpha$-Synuclein, a connection that has not been thus far described. TRAP1 was also described as having constitutive serine phosphorylation, that could be increased upon PINK1 signaling/ hydrogen peroxide treatment of PC12 cells (other forms of 


\section{DISCUSSION}

phosphorylation were not investigated). ${ }^{103}$ As the phosphorylation status of TRAP1 is thus implicated as important for TRAP1s protective effects, putative phosphorylation sites were mutated. As phosphorylation sites were not previously specified, the theoretic sites of T494 and Y498 were mutated. ${ }^{287}$

When expressed alone, [D158N] enhanced sensitivity to rotenone vs. wt TRAP1. It also showed a less prominent enhancement of Complex I activity vs. wt TRAP1. Similarly, when expressed with $\alpha$-Synuclein[A53T], the [D158N] mutant showed a less efficacious rescue of both oxidative stress and Complex I activity. Moreover, in comparison to wt, the [D158N] mutant showed limited evidence of protein-protein interaction with $\alpha$ Synuclein[A53T]. The pattern of phosphorylation observed was quite different than that seen for wt TRAP1. wt TRAP1 showed an enhancement of serine phosphorylation only during oxidative stress and $\alpha$-Synuclein[A53T] co-expression. This suggests that serine phosphorylation, whether directly due to PINK1 or other kinases, occurs specifically in the context of $\alpha$-Synuclein or alternately, during times of pronounced stress. In contrast, the mutant showed heavy serine phosphorylation under both resting and oxidative stress conditions. Oxidative stress additionally resulted in both threonine and tyrosine phosphorylation. In contrast, wt TRAP1 showed only minimal threonine phosphorylation under both conditions. This data jointly suggests that ATPase domain mutation results in significant alteration to TRAP1 regulation. The lack of protein-protein interaction and the absence of increased serine phosphorylation in the presence of $\alpha$-Synuclein[A53T] suggests that the ATPase domain is key to TRAP1's interaction and functional effects in regards to $\alpha$ Synuclein[A53T]. The Pridgeon et al paper had demonstrated that increased serine phosphorylation of TRAP1, downstream of PINK1, was key to its protective functions in the face of oxidative stress. However, although TRAP1[D158N] showed more evidence of phosphorylation than wt TRAP1, it was less efficacious in protecting against oxidative stress. 


\section{DISCUSSION}

This suggests a hypothesis regarding the role of phosphorylation in regulating mutant and wt

TRAP1 function. Upstream kinases that normally phosphorylate may function via a negative feedback cycle, such that following wt TRAP1 phosphorylation and its successful execution of downstream function, downstream mediators turn off the upstream kinases. In the case of the $[\mathrm{D} 158 \mathrm{~N}]$ mutant, its lack of downstream effect results in a lack of inhibition on the upstream kinases and thus enhanced phosphorylation of TRAP1 results, to no effect. In addition, the presence of threonine phosphorylation of [D158N] suggests that mutation of the ATPase domain changes the overall regulation of the protein, such that additional kinases result in aberrant and deleterious phosphorylation of the protein. Further investigation is necessary to either confirm or dismiss these hypotheses and to identify all of the kinases and upstream mediators that are involved in TRAP1 regulation, especially in the context of $\alpha$ Synuclein[A53T]. Indeed, in the presence of $\alpha$-Synuclein[A53T], the [D158N] mutation appears to act as a dominant negative mutant in regards to Complex II activity, as Complex II activity had previously not been affected by $\alpha$-Synuclein[A53T]. These alterations to TRAP1 function together suggest that the ATPase domain is key to TRAP1 function, involved in both reaction to general oxidative stress and ETC component moderation. More data is needed to better clarify the [D158N] effect. Such experiments were beyond the scope of the present study.

Based on Western data, TRAP1 appears to have minor constitutive threonine phosphorylation that was more evident in the presence of $\alpha$-Synuclein[A53T] when under oxidative stress. Threonine phosphorylation on the [T494A] mutation was not observed. Lack of strong staining for threonine phosphorylation with the mutant suggests a relative paucity of other P-threonine sites. While the [T494A] showed a slightly greater sensitivity to oxidative stress than wt TRAP1, it could still rescue the $\alpha$-Synuclein[A53T]-induced oxidative stress sensitivity. This suggests the effect of threonine phosphorylation on oxidative stress response 


\section{DISCUSSION}

is not pronounced. However, data indicates that the T494 phosphorylation site may be important for moderation of both native TRAP1 and $\alpha$-Synuclein[A53T] effect on the ETC Complexes. Expression of [T494A] alone did not enhance Complex I activity as per wt TRAP1 and moreover decreased Complex IV activity. Rescue of $\alpha$-Synuclein-induced Complex I defect was not achieved with the [T494A] mutant. Moreover, an additional decrease in Complex II and IV was recorded when $\alpha$-Synuclein[A53T] was expressed in combination with [T494A]. Notably, [T494A] showed limited evidence of serine phosphorylation. This suggests that alteration of the T494 site additionally effects serine phosphorylation, which seems important to rescue of $\alpha$-Synuclein-associated toxicity. Together this data indicates a key effect of T494 site on TRAP1-ETC interaction.

Evidence of constitutive tyrosine phosphorylation of TRAP1 was observed, with an apparent increase in tyrosine phosphorylation upon $\alpha$-Synuclein[A53T] expression under both resting and oxidative stress conditions. The effect of [Y498G] on phosphorylation is unclear, as a tyrosine phosphorylation band was still observed under resting conditions, but not after oxidative stress treatment (Fig. IV-41). A decrease in tyrosine phosphorylation during oxidative stress also implicates aberrant interaction of mutant TRAP1 and yet to be identified protein phosphatases. However, while the effect cannot be finally ascribed to a change in tyrosine phosphorylation, the mutation of Y498 did indeed have an effect on TRAP1 function. In comparison to $[\mathrm{D} 158 \mathrm{~N}] /[\mathrm{T} 494 \mathrm{~A}]$, the $[\mathrm{Y} 498 \mathrm{G}]$ mutation appeared to be silent in the context of interaction with ETC Complexes. However, [Y498G] reduced survival after oxidative stress and reduced total ATP content, in comparison to wt TRAP1 alone. This suggests that the T494 and Y498 sites differentially modulate TRAP1 function, with one site important for ETC interaction and the other involved mediating the response to oxidative stress and apoptosis signaling. Further investigation is require to clarify the structural effect of Y498 mutation. 


\section{DISCUSSION}

Downstream targets of TRAP1, such as Cytochrome c, were not investigated for the mutants, but would be seem to be an interesting line of investigation. As for known upstream kinases, only PINK1 has been identified. Thus, it is possible that phosphorylation of T494 site is due to PINK1 action and thus that PINK1 function can be tied to the ETC function, but this remains to be confirmed. Unfortunately, only recently were additional serine phospho-sites defined $^{287}$, and thus these sites, also potential downstream targets of PINK1, were not mutated. As the Y498 also seems to be functionally important, an upstream tyrosine kinase would also seem to be implicated in the TRAP1 signaling pathway. Alternatively, it may be that alteration of the amino acid has a functional consequence on 3D structure that also affects TRAP1 function. More detailed studies are needed to clarify this point. In conclusion, both the ATPase domain and phosphorylation modification have been implicated as key to TRAP1 function, but both require more detailed investigation.

\subsection{Significance of TRAP1- $\alpha$ - Synuclein[A53T] interaction in the context of PD pathogenesis}

This study closely connects the mitochondrial protein TRAP1 to $\alpha$-Synuclein, as TRAP1 reduction/overexpression has a direct impact on $\alpha$-Synuclein[A53T]-induced toxicity. Thus, a mitochondrial-centric theory of toxicity can be hypothesized. $\alpha$-Synuclein[A53T] overexpression causes multiple mitochondrial deficits resulting in reduction of ATP production. This leads to inhibition of key cell functions including ubiquitylation and proteasome function. At the same time, ETC deficits, such as that noted for Complex I, may increase generation of ROS and thereby increase the number of oxidated and misfolded proteins. These two effects together result in an increase in protein accumulation and aggregation. Decrease of TRAP1 levels further enhances this effect. It can thus be hypothesized that the alteration to cytosolic aggregation of $\alpha$-Synuclein[A53T] noted in $C$. 


\section{DISCUSSION}

elegens due to TRAP1 decrease is secondary to a loss of protection against $\alpha$ Synuclein[A53T]-induced mitochondrial deficits. ${ }^{107}$

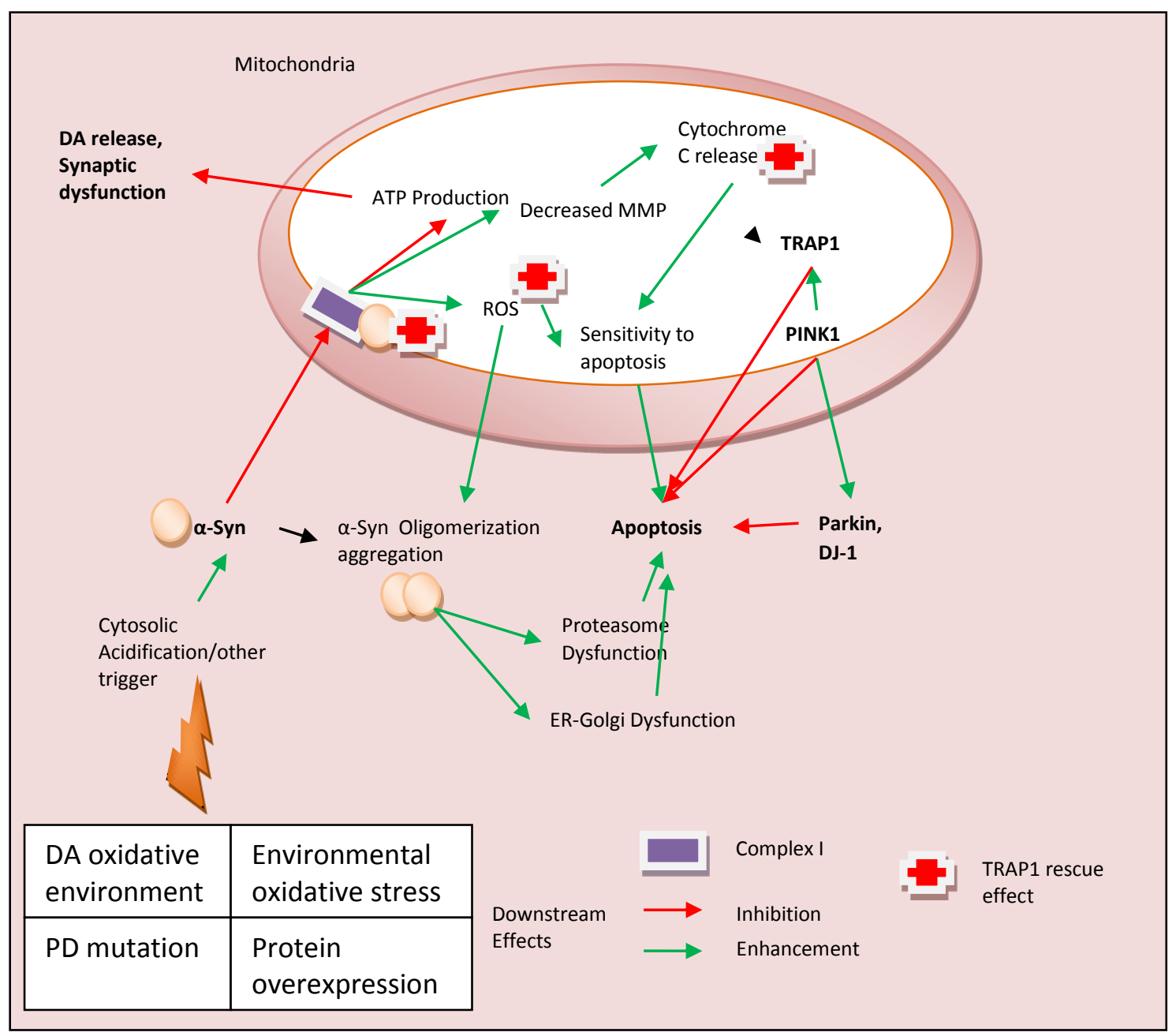

Fig.V-1. Schema for Understanding $\alpha$ - Synuclein[A53T] Mitochondrial PD Pathology. See text for detailed description. MMP=mitochondrial membrane potential.

Similar models also exist for toxicity ensuing from mutation or deletion of PINK1 or Parkin. Indeed, on the basis of TRAP1 suppression of the $\alpha$-Synuclein phenotype and the direct interaction of TRAP1 and $\alpha$-Synuclein[A53T] described in this study, $\alpha$ Synuclein[A53T] can be placed within the PINK1 pathway (see Fig.V-1). In the PD brain, as a result of an unknown oxidative stress trigger/DA oxidative environment, $\alpha$-Synuclein can begin to form oligomeric structures within the cytoplasm, that cause damage to internal organelles and that additionally move into the mitochondria. $\alpha$-Synuclein entry into mitochondria may have toxic effects, but key is a direct or downstream interaction with 


\section{DISCUSSION}

Complex I, resulting in its decreased activity. Complex I deficit can reduce mitochondrial membrane potential and ATP levels, and eventually increases ROS production. This results in additional functional changes, including a decrease in DA release ${ }^{288}$, increased sensitivity to apoptosis and Cytochrome c release. In sum, cell organelles and proteins may be further damaged, aggravating $\alpha$-Synuclein[A53T] entry into the mitochondria and aggregation within the cytosol. A positive feedback cycle ensues and neuron death is the end result. TRAP1, acting downstream of both PINK1 and perhaps other, as yet undetermined signaling molecules, counteracts the key toxic effect of $\alpha$-Synuclein[A53T], that being Complex I inhibition. In addition, it can inhibit downstream effects of ROS formation and Cytochrome c release, by as yet undetermined mechanisms. While there are still many unknowns, an additional linkage between the genes involved in PD adds an additional layer of understanding to PD pathogenesis. Such knowledge moves the field closer to a better identification of molecular targets able to halt disease progression and thus identify worthy clinical targets. 


\section{APPENDIX METHODS}

\section{A1. Appendix Methods.}

Fig. A1-1. Protein sequencing alignment data generated using ClustalW showing conserved aspartic acid in HSP90 proteins and in human TRAP1 ATPase domain, position [D158N]. http://www.e bi.ac.uk/Tools/clustalw2/index.html

\begin{tabular}{|c|c|}
\hline & E TQTQDQPMEEEEVETFAFQAE I AQLMSL I INTFYSNKE IFLREL ISNS \\
\hline & EKS---AQPVMEEEVETFAF QAE IAQLMSL I INTFYSNKE IFLREL ISNS \\
\hline & ------ MPEEV ----- HHGEE EVETFAF QAE I A QLMSL I INTFYSNKE IFLREL ISNA \\
\hline & ------ MTSKD------ E TP DQEVYAFNA D I S QL L SL I INAFYSNKE IFLREL ISNA \\
\hline & - - - - - - \\
\hline \multirow[t]{2}{*}{ Q12931 } & TQTAEDKEEPLHSI ISSTESVQGSTSKHEFQAETKKLLD IVARSLYSEKEVF IREL ISNA \\
\hline & $:: \quad: *^{* *}:{ }^{* *}:{ }^{*}:{ }^{* * * * *}:$ \\
\hline & SDALDKIRYESLTDPSKLDSGKELH INL I PNKQDRTLT VDT IGMTKADL INNLGTIAK \\
\hline 74 & SDALDKIRYESLTDPSKLDSCKDLKIEL I PDQKERTLT IDT I GMTKADL INNLGTIAK \\
\hline & SDALDKIRYESLTDPSKLDSGKELKID I IPNPQERTLT,VDT I GMTKADL INNLGTIAK \\
\hline 24 & SDALEKIRYEA IKDPKQIEDQPDYYIRLYADKNNNTLT EDS I GMTKADLVNNLGTIAK \\
\hline$P 02829$ & SDALDKIRYKSLSDPKQLETEPDLF IRITPKPEQKVLE RDS IGMTKAELINNLGTIAK \\
\hline \multirow[t]{2}{*}{ Q12931 } & SDALEKLRHKLVSDGQALP---EMEIHLQTNAEKGTIT QDT IGMTQEELVSNLGTIAR \\
\hline & 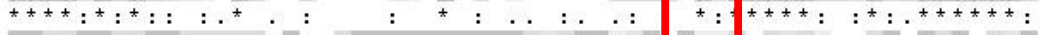 \\
\hline & SGTKAFMEALQAGADIS--MIGQFGVGFYSAYLVAEKV TIINHNDDE- -QYAWESSAGG \\
\hline & SGTKAFMEAL QAGAD IS- -MIGQFGVGFYSAYLVAEKVTVITKHNDDE--QYIWESAAGG \\
\hline & SGTKAFMEAL QAGAD IS--MI GQF GVGFYSAYLVAEKVVVITKHNDDE--QYA WESSAGG \\
\hline & SGTRAFMEALQA GSDMS--MIGQFGVGFYSAYLVADKVTVVSKNNADD--QYVWESTASG \\
\hline P02829 & SGTKAFMEALSAGADVS--MIGQFGVGFYSLFLVADRVQVISKSNDDE--QYIWESMAGG \\
\hline \multirow[t]{2}{*}{ Q12931 } & SGSKAFLDALQNQAEASSKI I GQF GVGFYSAFMVADRVEVYSRSAAPGSLGYQWLSDGSG \\
\hline & 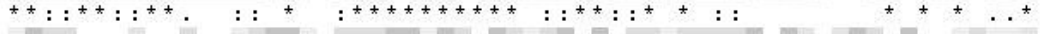 \\
\hline & \\
\hline
\end{tabular}

$\begin{array}{ll}52 & \text { HS90A_HUMAN } \\ 49 & \text { H9OA1_DANRE } \\ 47 & \text { HS90B_HUMAN } \\ 45 & \text { HSP90_THEPA } \\ 38 & \text { HSP82_YEAST } \\ 120 & \text { TRAP1_HUMAN } \\ 112 & \text { HS90A_HUMAN } \\ 109 & \text { H90A1_DANRE } \\ 107 & \text { HS90B_HUMAN } \\ 105 & \text { HSP90_THEPA } \\ 98 & \text { HSP82_YEAST } \\ 177 & \text { TRAP1_HUMAN } \\ 168 & \text { HS90A_HUMAN } \\ 165 & \text { H90A1_DANRE } \\ 163 & \text { HS90B_HUMAN } \\ 161 & \text { HSP90_THEPA } \\ 154 & \text { HSP82_YEAST } \\ 237 & \text { TRAP1_HUMAN } \\ & \\ 2.27 & \text { HSOMA_H HMAN }\end{array}$

Fig. A1-2. Protein sequencing alignment data generated using ClustalW showing conserved aspartic acid at position [D158N] in human TRAP1. http://www.ebi.ac.uk/Tools/clustalw2/index.html

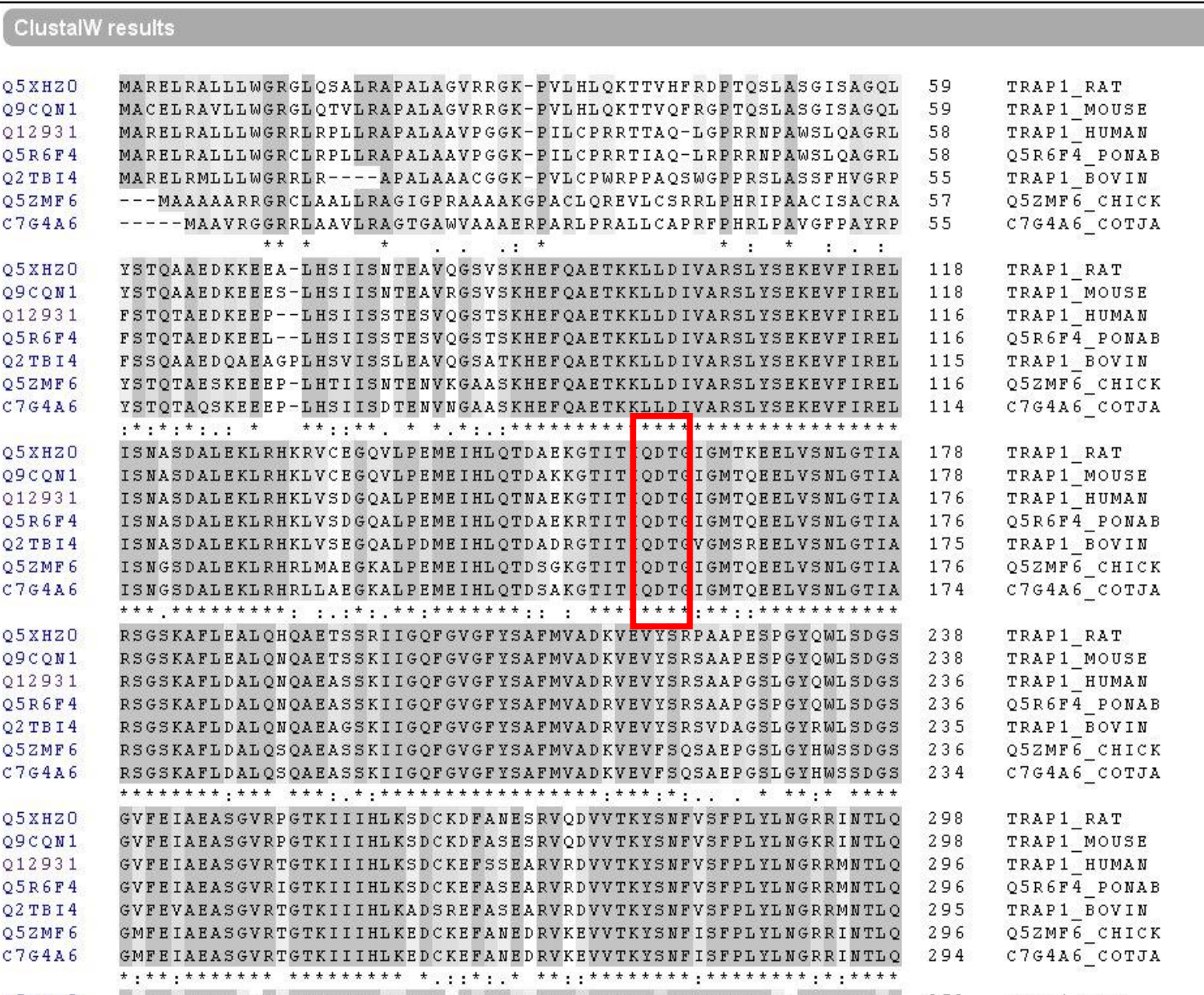




\section{APPENDIX METHODS}

Fig. A1-3. TRAP1 domain data and putative phosphorylation sites based on sequence data. Data from http://www.phosphosite.org/proteinAction.do?id=4177\&showAllSites=false. At the time of mutagenesis design, only T494 and Y498 sites were described and therefore experimentally targeted. TRAP1 also known as HSP75.

Click here to view other types of protein modifications

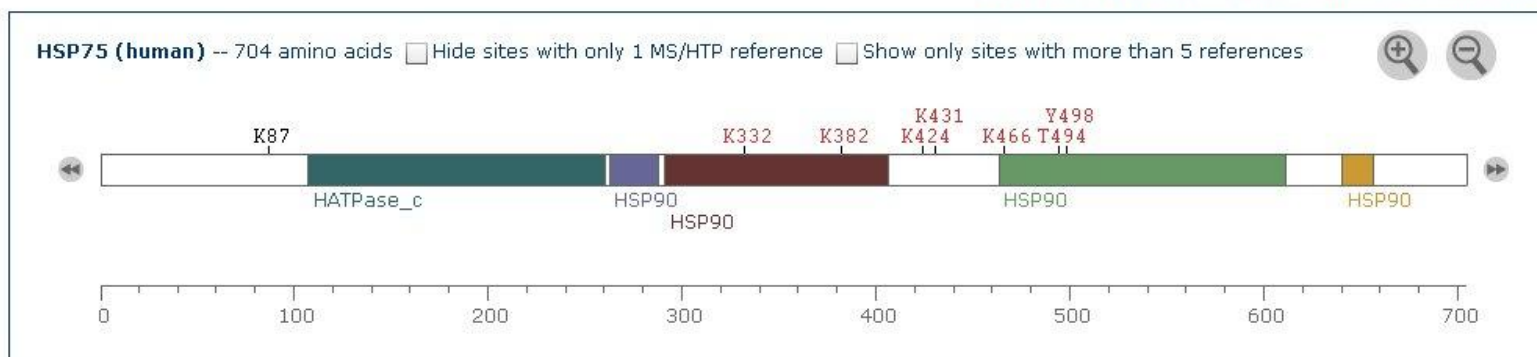

Modification Sites in Parent Protein, Orthologs, and Isoforms

$\begin{array}{cc}\text { Refs LTP } & \text { Refs HTP } \\ 0 & 1 \\ 0 & 1 \\ 0 & 1 \\ 0 & 6 \\ 0 & 1 \\ 0 & 1 \\ 0 & 1 \\ 0 & >25\end{array}$

$\begin{array}{cc} & \text { hUman } \\ \text { K87 } & \text { SVQGSTSKHEF QAET } \\ \text { K332 } & \text { PRYTLHYKTDAPLHI } \\ \text { K382 } & \text { KATDILPKWLRFIRG } \\ \text { K424 } & \text { VLQORLIKFFIDQSK } \\ \text { K431 } & \text { KFFIDQSKKD AEKYA } \\ \text { K466 } & \text { EVKEDIAKLLRYESS } \\ \text { T494 } & \text { ASRMRAGTRUIYYLC } \\ \text { Y498 } & \text { RAGTRHIYYLCAPYR }\end{array}$

mouse

$\begin{array}{ll}\text { K89 } & \text { AVRGSUSKHEFQHET } \\ \text { K334 } & \text { PRFTLHYKTDAPLHI } \\ \text { K384 } & \text { KAADILPKWLRFIRG } \\ \text { K426 } & \text { VLQORLIKFFIDQSK } \\ \text { K433 } & \text { KFFIDQSKKD AEKY } \\ \text { K468 } & \text { DIKEDIAKLLRYESS } \\ \text { T496 } & \text { ASRMOAGTRUIYYLC } \\ \text { Y500 } & \text { QHGTRWIYYLCAPHR }\end{array}$

$\underline{\text { rat }}$

\begin{tabular}{ll} 
K89 & AVOGSUSKHEFOAET \\
K334 & PRFILHYKTDAPLHI \\
K384 & KATDILPKWLRFVRG \\
K426 & VLOQRLIKFFIDQSK \\
K433 & KFFIDOSKKDAEKYA \\
K468 & DIKEDIAKLLRYESS \\
T496 & ASRMOAGTRWIYYLC \\
\hline
\end{tabular}

Y500 OAGTRHIYYLCAPHR

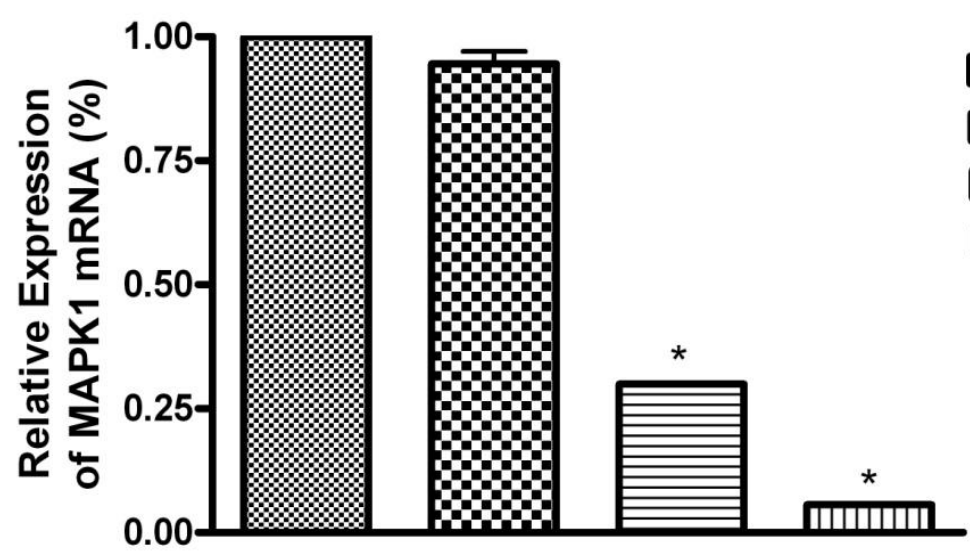

Untransfected Control $\infty$ Negative SiRNA Control ए5 nM siMAPK1 밈 15 nM siMAPK1

Fig. A1-4. MAPK knockdown in HEK293 following siRNA treatment: confirmation using real time PCR. MAPK expression was compared to $\beta$-actin expression. Both $5 \mathrm{nM}$ and $15 \mathrm{nM}$ siRNA concentrations were effective in reducing MAPK1(*, $\mathrm{p}<0.001$ vs. Controls). Treatment with negative siRNA (scrambled sequence) did not alter MAPK expression ( $\mathrm{p}=\mathrm{ns}$ vs. Untransfected control). 
Validation of siRNA delivery: MAPK knockdown at protein level

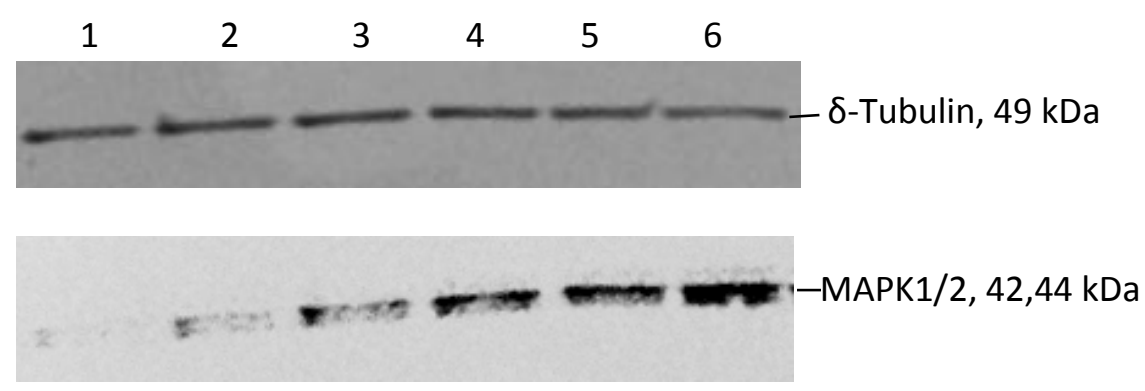

Fig A1-5. MAPK knockdown in HEK293 following siRNA treatment: confirmation using Western blotting. MAPK samples: 1) $20 \mathrm{nM}$ siRNA 2) $15 \mathrm{nM}$ siRNA 3) $5 \mathrm{nM}$ siRNA 4)1 $\mathrm{nM}$ siRNA 5) Scramble siRNA 6) Untreated. Expression compared to reference protein expression, $\delta$-Tubulin. 


\section{APPENDIX RESULTS}

\section{A2. Appendix Results.}

Table 1. Head DA Measurements from Primary Screen. **= 'high' DA; *= 'low' DA; SL= semi lethal, no HPLC data collected; L=lethal, no HPLC data collected. High and low values are means of 2 measurements, all other values single measurements.

\begin{tabular}{|c|c|c|c|c|}
\hline Cross & BL Stock & Symbol & Deleted segment & $\begin{array}{lcr}\text { Head } & \text { DA } & (4 \\
\text { week } & \text { as } \% & 1 \\
\text { week } & & \end{array}$ \\
\hline 1 & 1329 & $\mathrm{Df}(1) \mathrm{BA} 1$ & $1 \mathrm{~A} 1 ; 2 \mathrm{~A}$ & $116^{* *}$ \\
\hline 2 & 1546 & Df(1)sc-J4 & 1B2-14;3A3 & 73.23 \\
\hline 3 & 936 & $\operatorname{Df}(1) 64 \mathrm{c} 18$ & $2 \mathrm{E} 1-2 ; 3 \mathrm{C} 2$ & SL \\
\hline 4 & 935 & Df(1)JC19 & $2 \mathrm{~F} 6 ; 3 \mathrm{C} 5$ & SL \\
\hline 5 & 729 & $\mathrm{Df}(1) \mathrm{N}-8$ & $3 \mathrm{C} 2-3 ; 3 \mathrm{E} 3-4$ & SL \\
\hline 6 & 939 & $\operatorname{Df}(1) \mathrm{dm} 75 \mathrm{e} 19$ & $3 \mathrm{C} 11 ; 3 \mathrm{E} 4$ & SL \\
\hline 7 & 940 & $\operatorname{Df}(1) \mathrm{A} 113$ & 3D6-E1;4F5 & 65.81 \\
\hline 8 & 944 & Df(1)JC70 & 4C15-16;5A1-2 & 74.37 \\
\hline 9 & 945 & Df(1)C149 & $5 \mathrm{~A} 8-9 ; 5 \mathrm{C} 5-6$ & 74.15 \\
\hline 10 & 946 & Df(1)N73 & 5C2;5D5-6 & SL \\
\hline 11 & 5281 & $\mathrm{Df}(1) \mathrm{dx} 81$ & 5C3-10;6C3-12 & $\mathrm{L}$ \\
\hline 12 & 9625 & Df(1)ED6878 & $6 \mathrm{C} 12 ; 6 \mathrm{D} 8$ & 81.20 \\
\hline 13 & 3196 & Df(1)Sxl-bt & 6E2;7A6 & $176^{* *}$ \\
\hline 14 & 948 & Df(1)ct-J4 & $7 \mathrm{~A} 2-3 ; 7 \mathrm{C} 1$ & $26.82 *$ \\
\hline 15 & 3221 & $\mathrm{Df}(1) \mathrm{ct} 4 \mathrm{~b} 1$ & 7B2-4;7C3-4 & 90.10 \\
\hline 16 & 949 & $\operatorname{Df}(1) \mathrm{C} 128$ & 7D1;7D5-6 & 89.20 \\
\hline 17 & 950 & Df(1)RA2 & 7D10;8A4-5 & 75.62 \\
\hline 18 & 951 & Df(1)KA14 & 7F1-2;8C6 & 70.25 \\
\hline 19 & 3651 & Df(1)lz-90b24 & 8B5-6;8D8-9 & 87.73 \\
\hline 20 & 952 & $\operatorname{Df}(1) \mathrm{C} 52$ & 8E;9C-D & $138.52 * *$ \\
\hline 21 & 954 & Df(1)v-L15 & 9B1-2;10A1-2 & 87.17 \\
\hline 22 & 3560 & Df(1)v-N48 & 9F;10C3-5 & 70.02 \\
\hline 23 & 959 & Df(1)HA85 & 10C1-2;11A1-2 & 53.60 \\
\hline 24 & 962 & Df(1)N105 & 10F7;11D1 & $107.59 * *$ \\
\hline 25 & 967 & $\mathrm{Df}(1) \mathrm{C} 246$ & 11D-E;12A1-2 & 81.70 \\
\hline 26 & 727 & $\mathrm{Df}(1) \mathrm{g}$ & 12A3-10;12E9 & SL \\
\hline 27 & 998 & Df(1)RK2 & 12D2-E1;13A2-5 & 80.12 \\
\hline 28 & 1039 & $\operatorname{Df}(1) \mathrm{RK} 4$ & 12F5-6;13A9-B1 & $105.78 * *$ \\
\hline 29 & 7339 & $\operatorname{In}(1) A C 2[L] A B[R]$ & $\begin{array}{l}\text { 9D5-E1;9E7-8 + 13B5 } \\
6 ; 13 \mathrm{E} 1-2\end{array}$ & 59.97 \\
\hline 30 & 3347 & $\operatorname{Df}(1) \operatorname{sd} 72 b$ & 13F1;14B1 & $113.74 * *$ \\
\hline 31 & 2099 & Df(1)XR38 & $14 \mathrm{~A} ; 15 \mathrm{D}$ & 85.73 \\
\hline 32 & 9905 & Df(1)ED7364 & 14A8;14C6 & 60.45 \\
\hline 33 & 125 & Df(1)4b18 & 14B8;14C1 & $47.44 *$ \\
\hline 34 & 3217 & $\operatorname{Tp}(1 ; 2) \mathrm{r}[+] 75 \mathrm{c}$ & 14B13;15A9 & 51.75 \\
\hline 35 & 5272 & $\mathrm{Df}(1) \mathrm{r}-\mathrm{D} 1$ & 14C5-6;15B1 & 67.89 \\
\hline 36 & 4741 & Df(1)B25 & 15D3;16A4-6 & $110.36^{* *}$ \\
\hline 37 & 4953 & Df(1)BK10 & $16 \mathrm{~A} 2 ; 16 \mathrm{C} 7-10$ & $\mathrm{~L}$ \\
\hline 38 & 6217 & Df(1)RR79 & $16 \mathrm{C} ; 16 \mathrm{~F}$ & SL \\
\hline 39 & 970 & $\operatorname{Df}(1) \mathrm{N} 19$ & 17A1;18A2 & 63.38 \\
\hline 40 & 7754 & Df(1)Exel6291 & 18A2;18A2 & 68.33 \\
\hline 41 & 971 & Df(1)JA27 & 18A5;18D & 72.73 \\
\hline 42 & 7721 & Df(1)Exel6253 & 18D13;18F2 & 98.62 \\
\hline 43 & 972 & Df(1)HF396 & 18E1-2;20 & 64.94 \\
\hline
\end{tabular}


APPENDIX RESULTS

\begin{tabular}{|c|c|c|c|c|}
\hline 44 & 977 & Df(1)DCB1-35b & 19F1-2;20E-F & 71.23 \\
\hline 45 & 3714 & Df(1)A209 & $20 \mathrm{~A} ; 20 \mathrm{~F}$ & 82.49 \\
\hline 46 & 3638 & Df(2L)net-PMF & 21A1;21B7-8 & $131.30 * *$ \\
\hline 47 & 8672 & Df(2L)BSC106 & 21B7;21C2 & $114.21 * *$ \\
\hline 48 & 6283 & $\mathrm{Df}(2 \mathrm{~L}) \mathrm{BSC} 4$ & 21B7-C1;21C2-3 & 87.67 \\
\hline 49 & 6608 & Df(2L)BSC16 & 21C3-4;21C6-8 & 59.30 \\
\hline 50 & 3084 & $\operatorname{Df}(2 \mathrm{~L})$ ast2 & 21D1-2;22B23 & $47.06^{*}$ \\
\hline 51 & 3133 & $\operatorname{Df}(2 \mathrm{~L}) \mathrm{dp}-79 \mathrm{~b}$ & 22A2-3;22D5-E1 & $180.85 * *$ \\
\hline 52 & 7144 & Df(2L)BSC37 & 22D2-3;22F1-2 & 66.97 \\
\hline 53 & 6648 & $\operatorname{Df}(2 \mathrm{~L}) \mathrm{dpp}[\mathrm{d} 14]$ & 22E4-F2;22F3-23A1 & 62.71 \\
\hline 54 & 90 & $\mathrm{Df}(2 \mathrm{~L}) \mathrm{C} 144$ & 22F4-23A1;23C2-4 & 53.94 \\
\hline 55 & 1567 & $\mathrm{Df}(2 \mathrm{~L}) \mathrm{JS} 17$ & 23C1-2;23E1-2 & 69.56 \\
\hline 56 & 6875 & Df(2L)BSC28 & 23C5-D1;23E2 & 65.44 \\
\hline 57 & 6965 & Df(2L)BSC31 & 23E5;23F4-5 & 83.95 \\
\hline 58 & 6507 & $\mathrm{Df}(2 \mathrm{~L}) \mathrm{drm}-\mathrm{P} 2$ & 23F3-4;24A1-2 & 88.92 \\
\hline 59 & 5330 & Df(2L)ed1 & 24A2;24D4 & 77.50 \\
\hline 60 & 693 & Df(2L)sc19-8 & $24 \mathrm{C} 2-8 ; 25 \mathrm{C} 8-9$ & $198.92 * *$ \\
\hline 61 & 9270 & Df(2L)ED250 & 24F4;25A7 & 97.62 \\
\hline 62 & 8835 & $\operatorname{Df}(2 \mathrm{~L}) \mathrm{BSC} 110$ & $25 \mathrm{C} 1 ; 25 \mathrm{C} 4$ & 79.38 \\
\hline 63 & 8674 & Df(2L)BSC109 & $25 \mathrm{C} 4 ; 25 \mathrm{C} 8$ & 59.82 \\
\hline 64 & 7497 & Df(2L)Exel6011 & $25 \mathrm{C} 8 ; 25 \mathrm{D} 5$ & $\mathrm{~L}$ \\
\hline 65 & 781 & Df(2L)cl-h3 & 25D2-4;26B2-5 & 98.91 \\
\hline 66 & 490 & Df(2L)E110 & 25F3-26A1;26D3-11 & $205.68 * *$ \\
\hline 67 & 6299 & Df(2L)BSC5 & 26B1-2;26D1-2 & 74.35 \\
\hline 68 & 6338 & Df(2L)BSC6 & 26D3-E1;26F4-7 & 56.21 \\
\hline 69 & 6374 & Df(2L)BSC7 & 26D10-E1;27C1 & 90.16 \\
\hline 70 & 2414 & $\operatorname{Df}(2 \mathrm{~L}) \operatorname{spd}[\mathrm{j} 2]$ & $27 \mathrm{C} 1-2 ; 28 \mathrm{~A}$ & $47.48 *$ \\
\hline 71 & 5420 & Df(2L)Dwee1-W05 & $27 \mathrm{C} 2-3 ; 27 \mathrm{C} 4-5$ & 55.18 \\
\hline 72 & 4956 & Df(2L)XE-3801 & 27E2;28D1 & 65.00 \\
\hline 73 & 7147 & Df(2L)BSC41 & 28A4-B1;28D3-9 & 97.69 \\
\hline 74 & 9502 & Df(2L)BSC142 & 28C3;28D3 & 82.65 \\
\hline 75 & 140 & Df(2L)Trf-C6R31 & 28DE;28DE & 71.00 \\
\hline 76 & 179 & Df(2L)TE29Aa-11 & 28E4-7;29B2-C1 & $130.19 * *$ \\
\hline 77 & 8836 & $\operatorname{Df}(2 \mathrm{~L}) \mathrm{BSC} 111$ & 28F5;29B1 & $128.33^{* *}$ \\
\hline 78 & 9298 & Df(2L)ED611 & 29B4;29C3 & $180.83 * *$ \\
\hline 79 & 2892 & Df(2L)N22-14 & $29 \mathrm{C} 1-2 ; 30 \mathrm{C} 8-9$ & 81.97 \\
\hline 80 & 6478 & $\operatorname{Df}(2 \mathrm{~L}) \mathrm{BSC} 17$ & 30C3-5;30F1 & 83.15 \\
\hline 81 & 1045 & $\mathrm{Df}(2 \mathrm{~L}) \mathrm{Mdh}$ & 30D-30F;31F & $29.62 *$ \\
\hline 82 & 8469 & Df(2L)BSC50 & 30F5;31B1 & 92.26 \\
\hline 83 & 3366 & $\mathrm{Df}(2 \mathrm{~L}) \mathrm{J} 2$ & $31 \mathrm{~B} ; 32 \mathrm{~A}$ & 69.70 \\
\hline 84 & 9503 & Df(2L)BSC143 & 31B1;31D9 & $105.86 * *$ \\
\hline 85 & 7142 & Df(2L)BSC32 & 32A1-2;32C5-D1 & $31.34 *$ \\
\hline 86 & 9505 & Df(2L)BSC145 & $32 \mathrm{C} 1 ; 32 \mathrm{C} 1$ & 90.45 \\
\hline 87 & 7143 & Df(2L)BSC36 & 32D1;32D4-E1 & 98.14 \\
\hline 88 & 5869 & Df(2L)FCK-20 & 32D1;32F1-3 & 70.13 \\
\hline 89 & 3079 & $\operatorname{Df}(2 \mathrm{~L}) \operatorname{Prl}$ & 32F1-3;33F1-2 & 71.73 \\
\hline 90 & 6999 & Df(2L)BSC30 & 34A3;34B7-9 & 97.30 \\
\hline 91 & 9594 & Df(2L)BSC159 & 34B4;34C4 & $109.79 * *$ \\
\hline 92 & 3138 & $\mathrm{Df}(2 \mathrm{~L}) \mathrm{b} 87 \mathrm{e} 25$ & 34B12-C1;35B10-C1 & $102.12 * *$ \\
\hline 93 & 9506 & Df(2L)BSC147 & $34 \mathrm{C} 1 ; 34 \mathrm{C} 6$ & $27.51 *$ \\
\hline 94 & 3588 & Df(2L)TE35BC-24 & 35B4-6;35F1-7 & 84.37 \\
\hline 95 & 1491 & $\operatorname{Df}(2 \mathrm{~L}) \mathrm{r} 10$ & 35D1;36A6-7 & $109.20 * *$ \\
\hline
\end{tabular}


APPENDIX RESULTS

\begin{tabular}{|c|c|c|c|c|}
\hline 96 & 2583 & Df(2L)cact-255rv64 & 35F-36A;36D & $145.29 * *$ \\
\hline 97 & 420 & Df(2L)TW137 & 36C2-4;37B9-C1 & $104.59^{* *}$ \\
\hline 98 & 567 & Df(2L)pr-A16 & 37B2-12;38D2-5 & 95.69 \\
\hline 99 & 167 & $\operatorname{Df}(2 \mathrm{~L}) \mathrm{TW} 161$ & 38A6-B1;40A4-B1 & 79.06 \\
\hline 100 & 7531 & Df(2L)Exel6049 & 40A5;40D3 & 59.39 \\
\hline 101 & 9510 & Df(2L)BSC151 & 40A5;40E5 & $121.48^{* *}$ \\
\hline 102 & 4959 & $\mathrm{Df}(2 \mathrm{~L}) \mathrm{C}^{\prime}$ & h35;h38L & 97.30 \\
\hline 103 & 749 & $\operatorname{In}(2 \mathrm{R}) \mathrm{bw}[\mathrm{VDe} 2 \mathrm{~L}] \mathrm{Cy}[\mathrm{R}]$ & $\mathrm{h} 42-\mathrm{h} 43 ; 42 \mathrm{~A} 2-3$ & $122.02 * *$ \\
\hline 104 & 739 & $\mathrm{Df}(2 \mathrm{R}) \mathrm{M} 41 \mathrm{~A} 4$ & $41 \mathrm{~A} ; 41 \mathrm{~A}$ & 69.11 \\
\hline 105 & 1007 & Df(2R)nap9 & 42A1-2;42E6-F1 & $19.81 *$ \\
\hline 106 & 1888 & $\mathrm{Df}(2 \mathrm{R}) \mathrm{ST} 1$ & 42B3-5;43E15-18 & 57.62 \\
\hline 107 & 3368 & $\operatorname{Df}(2 \mathrm{R}) \mathrm{cn} 9$ & 42E;44C & 54.78 \\
\hline 108 & 198 & $\mathrm{Df}(2 \mathrm{R}) \mathrm{H} 3 \mathrm{C} 1$ & 43F;44D3-8 & $103.78 * *$ \\
\hline 109 & 201 & Df(2R)H3E1 & 44D1-4;44F12 & $49.94 *$ \\
\hline 110 & 3591 & $\mathrm{Df}(2 \mathrm{R}) \mathrm{Np} 5$ & 44F10;45D9-E1 & 76.92 \\
\hline 111 & 4966 & $\operatorname{Df}(2 \mathrm{R}) \mathrm{w} 45-30 \mathrm{n}$ & 45A6-7;45E2-3 & $47.2^{*}$ \\
\hline 112 & 6917 & Df(2R)BSC29 & 45D3-4;45F2-6 & 60.41 \\
\hline 113 & 9410 & $\mathrm{Df}(2 \mathrm{R}) \mathrm{BSC} 132$ & 45F6;46B4 & 50.18 \\
\hline 114 & 1743 & Df(2R)B5 & $46 \mathrm{~A} ; 46 \mathrm{C}$ & 50.47 \\
\hline 115 & 1702 & $\mathrm{Df}(2 \mathrm{R}) \mathrm{X} 1$ & 46C;47A1 & 85.22 \\
\hline 116 & 190 & $\mathrm{Df}(2 \mathrm{R}) \mathrm{en}-\mathrm{A}$ & 47D3;48B2 & 100.00 \\
\hline 117 & 1145 & $\operatorname{Df}(2 \mathrm{R}) \mathrm{en} 30$ & 48A3-4;48C6-8 & 88.98 \\
\hline 118 & 7145 & Df(2R)BSC39 & 48C5-D1;48D5-E1 & 57.56 \\
\hline 119 & 4960 & $\mathrm{Df}(2 \mathrm{R}) \mathrm{CB} 21$ & $48 \mathrm{E} ; 49 \mathrm{~A}$ & 88.54 \\
\hline 120 & 7146 & Df(2R)BSC40 & 48E1-2;48E2-10 & 86.52 \\
\hline 121 & 5879 & Df(2R)BSC3 & 48E12-F4;49A11-B6 & 85.45 \\
\hline 122 & 754 & $\mathrm{Df}(2 \mathrm{R}) \mathrm{vg}-\mathrm{C}$ & 49A4-13;49E7-F1 & $134.41 * *$ \\
\hline 123 & 442 & $\operatorname{Df}(2 \mathrm{R}) \mathrm{CX} 1$ & 49C1-4;50C23-D2 & 54.42 \\
\hline 124 & 6516 & Df(2R)BSC18 & 50D1;50D2-7 & 60.49 \\
\hline 125 & 7875 & Df(2R)Exel7130 & 50D4;50E4 & 95.84 \\
\hline 126 & 9496 & Df(2R)BSC134 & 50E1;50E6 & 95.86 \\
\hline 127 & 7876 & Df(2R)Exel7131 & 50E4;50F6 & 85.76 \\
\hline 128 & 6455 & $\mathrm{Df}(2 \mathrm{R}) \mathrm{BSC} 11$ & 50E6-F1;51E2-4 & 61.57 \\
\hline 129 & 3518 & $\mathrm{Df}(2 \mathrm{R}) \mathrm{Jp} 1$ & 51D3-8;52F5-9 & 56.04 \\
\hline 130 & 3520 & $\mathrm{Df}(2 \mathrm{R}) \mathrm{Jp} 8$ & 52F5-9;52F10-53A1 & $108.50 * *$ \\
\hline 131 & 7445 & Df(2R)BSC49 & 53D9-E1;54B5-10 & 54.56 \\
\hline 132 & 7414 & Df(2R)BSC44 & 54B1-2;54B7-10 & 59.78 \\
\hline 133 & 9596 & $\operatorname{Df}(2 \mathrm{R}) \mathrm{BSC} 161$ & 54B2;54B17 & $21.48^{*}$ \\
\hline 134 & 5574 & $\operatorname{Df}(2 \mathrm{R}) \mathrm{k} 10408$ & $54 \mathrm{~B} 16 ; 54 \mathrm{~B} 16$ & 66.44 \\
\hline 135 & 5680 & Df(2R)robl-c & 54B17-C4;54C1-4 & 54.56 \\
\hline 136 & 7441 & Df(2R)BSC45 & 54C8-D1;54E2-7 & $46.81 *$ \\
\hline 137 & 6779 & $\operatorname{Df}(2 \mathrm{R}) 14 \mathrm{H} 10 \mathrm{Y}-53$ & 54D1-2;54E5-7 & $125 * *$ \\
\hline 138 & 6780 & $\operatorname{Df}(2 \mathrm{R}) 14 \mathrm{H} 10 \mathrm{~W}-35$ & 54E5-7;55B5-7 & 87.61 \\
\hline 139 & 1547 & $\mathrm{Df}(2 \mathrm{R}) \mathrm{PC} 4$ & $55 \mathrm{~A} ; 55 \mathrm{~F}$ & 51.16 \\
\hline 140 & 757 & $\operatorname{Df}(2 \mathrm{R}) \mathrm{P} 34$ & 55E2-4;56C1-11 & $49.94 *$ \\
\hline 141 & 6866 & $\operatorname{Df}(2 \mathrm{R}) \mathrm{BSC} 26$ & 56C4;56D6-10 & 83.30 \\
\hline 142 & 6647 & $\mathrm{Df}(2 \mathrm{R}) \mathrm{BSC} 22$ & 56D7-E3;56F9-12 & 92.78 \\
\hline 143 & 3467 & $\mathrm{Df}(2 \mathrm{R}) \mathrm{AA} 21$ & 56F9-17;57D11-12 & 73.05 \\
\hline 144 & 7896 & Df(2R)Exel7162 & 56F11;56F16 & 93.58 \\
\hline 145 & 6609 & Df(2R)BSC19 & 56F12-14;57A4 & 64.89 \\
\hline 146 & 5246 & $\operatorname{Df}(2 \mathrm{R}) \mathrm{Egfr} 5$ & 57D2-8;58D1 & 80.97 \\
\hline
\end{tabular}


APPENDIX RESULTS

\begin{tabular}{|c|c|c|c|c|}
\hline 147 & 282 & $\operatorname{Df}(2 \mathrm{R}) \mathrm{X} 58-12$ & 58D1-2;59A & $126.3 * *$ \\
\hline 148 & 3909 & $\mathrm{Df}(2 \mathrm{R}) 59 \mathrm{AD}$ & 59A1-3;59D1-4 & $41.00 *$ \\
\hline 149 & 7273 & $\mathrm{Df}(2 \mathrm{R}) \operatorname{vir} 130$ & 59B;59D8-E1 & $39.85 *$ \\
\hline 150 & 1682 & Df(2R)or-BR6 & 59D5-10;60B3-8 & 65.22 \\
\hline 151 & 9691 & $\mathrm{Df}(2 \mathrm{R}) \mathrm{BSC} 155$ & 60B8;60C4 & 52.43 \\
\hline 152 & 2604 & $\mathrm{Df}(2 \mathrm{R}) \mathrm{Px} 2$ & 60C5-6;60D9-10 & 80.41 \\
\hline 153 & 9069 & $\operatorname{Df}(2 \mathrm{R}) \mathrm{ED} 4065$ & 60C8;60E8 & 78.79 \\
\hline 154 & 2471 & Df(2R)M60E & 60E2-3;60E11-12 & 61.70 \\
\hline 155 & 4961 & $\operatorname{Df}(2 \mathrm{R}) \mathrm{Kr} 10$ & 60F1;60F5 & 67.92 \\
\hline 156 & 2577 & Df(3L)emc-E12 & 61A;61D3 & 75.24 \\
\hline 157 & 439 & Df(3L)Ar14-8 & $61 \mathrm{C} 5-8 ; 62 \mathrm{~A} 8$ & 94.34 \\
\hline 158 & 7566 & Df(3L)Exel6087 & 62A2;62A6 & 94.96 \\
\hline 159 & 600 & Df(3L)Aprt-1 & 62A10-B1;62D2-5 & 52.25 \\
\hline 160 & 9693 & Df(3L)BSC181 & 62A11;62B7 & 85.20 \\
\hline 161 & 2400 & Df(3L)R-G7 & 62B4-7;62D5-E5 & 61.61 \\
\hline 162 & 6755 & $\operatorname{Df}(3 \mathrm{~L}) \mathrm{BSC} 23$ & 62E8;63B5-6 & 83.21 \\
\hline 163 & 3650 & $\operatorname{Df}(3 \mathrm{~L}) \mathrm{M} 21$ & 62F;63D & 83.58 \\
\hline 164 & 3649 & Df(3L)HR119 & $63 \mathrm{C} 2 ; 63 \mathrm{~F} 7$ & 40.70 \\
\hline 165 & 463 & Df(3L)GN34 & 63Е6-9;64A8-9 & 91.76 \\
\hline 166 & 3686 & Df(3L)GN24 & 63F6-7;64C13-15 & 50.24 \\
\hline 167 & 3096 & Df(3L)ZN47 & 64C;65C & $40.69 *$ \\
\hline 168 & 4393 & Df(3L)XDI98 & 65A2;65E1 & $24.71 *$ \\
\hline 169 & 6867 & $\mathrm{Df}(3 \mathrm{~L}) \mathrm{BSC} 27$ & 65D4-5;65E4-6 & 60.71 \\
\hline 170 & 6964 & Df(3L)BSC33 & 65E10-F1;65F2-6 & $41.51 *$ \\
\hline 171 & 1420 & $\mathrm{Df}(3 \mathrm{~L}) \mathrm{pbl}-\mathrm{X} 1$ & 65F3;66B10 & $107.10^{* * *}$ \\
\hline 172 & 5877 & $\mathrm{Df}(3 \mathrm{~L}) \mathrm{ZP} 1$ & 66A17-20;66C1-5 & 61.26 \\
\hline 173 & 1541 & Df(3L)66C-G28 & 66B8-9;66C9-10 & 50.24 \\
\hline 174 & 6460 & Df(3L)BSC13 & 66B12-C1;66D2-4 & 69.25 \\
\hline 175 & 3024 & Df(3L)h-i22 & 66D10-11;66E1-2 & $\mathrm{L}$ \\
\hline 176 & 4500 & Df(3L)Scf-R6 & 66E1-6;66F1-6 & $104.84 * *$ \\
\hline 177 & 7079 & Df(3L)BSC35 & 66F1-2;67B2-3 & $106.4^{* *}$ \\
\hline 178 & 997 & $\mathrm{Df}(3 \mathrm{~L}) \mathrm{AC} 1$ & 67A2;67D11-13 & SL \\
\hline 179 & 6471 & Df(3L)BSC14 & 67E3-7;68A2-6 & $191 * *$ \\
\hline 180 & 2611 & $\operatorname{Df}(3 \mathrm{~L}) \operatorname{vin} 5$ & 68A2-3;69A1-3 & 88.13 \\
\hline 181 & 2612 & $\operatorname{Df}(3 \mathrm{~L}) \operatorname{vin} 7$ & 68C8-11;69B4-5 & $26.11 *$ \\
\hline 182 & 5492 & Df(3L)eyg[C1] & 69A4-5;69D4-6 & 57.50 \\
\hline 183 & 6456 & Df(3L)BSC10 & \begin{tabular}{|l|}
$69 \mathrm{D} 4-5 ; 69 F 5-7$ \\
\end{tabular} & 64.96 \\
\hline 184 & 6457 & Df(3L)BSC12 & 69F6-70A1;70A1-2 & 84.57 \\
\hline 185 & 4366 & $\operatorname{In}(3 \mathrm{LR}) \mathrm{C} 190[\mathrm{~L}] \mathrm{Ubx}[42 \mathrm{TR}]$ & 70A1-2;70C $3-4$ & 66.04 \\
\hline 186 & 3124 & Df(3L)fz-GF3b & 70C1-2;70D4-5 & $144.89 * *$ \\
\hline 187 & 3126 & Df(3L)fz-M21 & 70D2-3;71E4-5 & 74.66 \\
\hline 188 & 6551 & Df(3L)XG5 & 71C2-3;72B1-C1 & 96.84 \\
\hline 189 & 3640 & Df(3L)brm11 & 71F1-4;72D1-10 & 77.29 \\
\hline 190 & 2993 & Df(3L)st-f13 & 72C1-D1;73A3-4 & $100.52 * *$ \\
\hline 191 & 2998 & $\operatorname{Df}(3 \mathrm{~L}) 81 \mathrm{k} 19$ & 73A3;74F & 67.16 \\
\hline 192 & 6411 & Df(3L)BSC8 & 74D3-75A1;75B2-5 & 79.73 \\
\hline 193 & 2608 & Df(3L)W10 & 75A6-7;75C1-2 & 97.35 \\
\hline 194 & 2990 & Df(3L)Cat & 75B8;75F1 & 87.51 \\
\hline 195 & 8082 & Df(3L)ED4782 & 75F2;76A1 & 50.53 \\
\hline 196 & 6754 & $\operatorname{Df}(3 \mathrm{~L}) \mathrm{fz} 2$ & 75F10-11;76A1-5 & 97.36 \\
\hline 197 & 6646 & Df(3L)BSC20 & 76A7-B1;76B4-5 & 84.23 \\
\hline
\end{tabular}


APPENDIX RESULTS

\begin{tabular}{|c|c|c|c|c|}
\hline 198 & 3617 & Df(3L)kto2 & 76B1-2;76D5 & $158.03 * *$ \\
\hline 199 & 5126 & $\operatorname{Df}(3 \mathrm{~L}) \mathrm{XS} 533$ & 76B4;77B & 53.28 \\
\hline 200 & 2052 & $\operatorname{Df}(3 \mathrm{~L}) \mathrm{rdgC}-\mathrm{co} 2$ & 77A1;77D1 & 91.55 \\
\hline 201 & 3127 & $\operatorname{Df}(3 \mathrm{~L}) \mathrm{ri}-79 \mathrm{c}$ & 77B-C;77F-78A & $105.56 * *$ \\
\hline 202 & 5878 & Df(3L)ri-XT1 & 77E2-4;78A2-4 & 58.66 \\
\hline 203 & 4429 & Df(3L)ME107 & 77F3;78C8-9 & 86.30 \\
\hline 204 & 4430 & $\operatorname{Df}(3 L) P c-2 q$ & 78C5-6;78E3-79A1 & 65.80 \\
\hline 205 & 8101 & Df(3L)ED4978 & 78D5;79A2 & 98.01 \\
\hline 206 & 9700 & Df(3L)BSC223 & 79A3;79B3 & 80.24 \\
\hline 207 & 23149 & Df(3L)BSC249 & 79B2;79D1 & 65.08 \\
\hline 208 & 4506 & Df(3L)Ten-m-AL29 & 79C1-3;79E3-8 & 83.41 \\
\hline 209 & 5951 & Df(3L)HD1 & 79D3-E1;79F3-6 & 69.31 \\
\hline 210 & 6649 & $\operatorname{Df}(3 \mathrm{~L}) \mathrm{BSC} 21$ & 79E5-F1;80A2-3 & 67.77 \\
\hline 211 & 1518 & Df(3R)ME15 & 81F3-6;82F5-7 & 84.26 \\
\hline 212 & 4787 & $\operatorname{Df}(3 R) 3-4$ & $82 \mathrm{~F} 3-4 ; 82 \mathrm{~F} 10-11$ & 76.56 \\
\hline 213 & 5694 & Df(3R)e1025-14 & 82F8-10;83A1-3 & 90.12 \\
\hline 214 & 7623 & Df(3R)Exel6144 & 83A6;83B6 & 93.38 \\
\hline 215 & 8103 & Df(3R)ED5177 & 83B4;83B6 & 68.47 \\
\hline 216 & 7443 & Df(3R)BSC47 & 83B7-C1;83C6-D1 & 76.81 \\
\hline 217 & 1990 & Df(3R)Tpl10 & 83C1-2;84B1-2 & 90.87 \\
\hline 218 & 2393 & Df(3R)WIN11 & 83E1-2;84A5 & 77.78 \\
\hline 219 & 1884 & $\operatorname{Df}(3 R) \operatorname{Scr}$ & 84A1-2;84B1-2 & $29.55^{*}$ \\
\hline 220 & 1842 & Df(3R)Antp17 & 84A5;84D9 & 70.67 \\
\hline 221 & 1968 & Df(3R)p712 & 84D4-6;85B6 & 86.48 \\
\hline 222 & 1962 & Df(3R)p-XT103 & $85 \mathrm{~A} 2 ; 85 \mathrm{C} 1-2$ & $46.60 *$ \\
\hline 223 & 6756 & Df(3R)BSC24 & 85C4-9;85D12-14 & 87.23 \\
\hline 224 & 1931 & Df(3R)by10 & 85D8-12;85E7-F1 & $45.14 *$ \\
\hline 225 & 7080 & Df(3R)BSC38 & 85F1-2;86C7-8 & 71.91 \\
\hline 226 & 3128 & Df(3R)M-Kx1 & $86 \mathrm{C} 1 ; 87 \mathrm{~B} 1-5$ & 54.85 \\
\hline 227 & 3003 & $\operatorname{Df}(3 \mathrm{R}) \mathrm{T}-32$ & 86Е2-4;87C6-7 & 65.89 \\
\hline 228 & 3007 & Df(3R)ry615 & 87B11-13;87E8-11 & 90.56 \\
\hline 229 & 1534 & $\mathrm{Tp}(3 ; \mathrm{Y}) \mathrm{ry} 506-85 \mathrm{C}$ & 87D1-2;88E5-6 & 75.70 \\
\hline 230 & 383 & Df(3R)ea & 88E7-13;89A1 & 70.57 \\
\hline 231 & 756 & $\operatorname{Df}(3 \mathrm{R}) \mathrm{sbd} 105$ & 88F9-89A1;89B9-10 & 85.10 \\
\hline 232 & 1920 & Df(3R)sbd104 & $89 \mathrm{~B} 5 ; 89 \mathrm{C} 2-7$ & 52.87 \\
\hline 233 & 1467 & $\mathrm{Df}(3 \mathrm{R}) \mathrm{P} 115$ & 89B7-8;89E7 & $182.22 * *$ \\
\hline 234 & 4431 & Df(3R)DG2 & 89E1-F4;91B1-B2 & $111.99 * *$ \\
\hline 235 & 3011 & Df(3R)Cha7 & 90F1-F4;91F5 & 92.33 \\
\hline 236 & 3012 & Df(3R)Dl-BX12 & 91F1-2;92D3-6 & $122.15^{* *}$ \\
\hline 237 & 4962 & Df(3R)H-B79 & 92B3;92F13 & $145.61 * *$ \\
\hline 238 & 7413 & Df(3R)BSC43 & 92F7-93A1;93B3-6 & 67.90 \\
\hline 239 & 2425 & Df(3R)e-N19 & 93B;94 & 76.45 \\
\hline 240 & 3340 & $\mathrm{Df}(3 \mathrm{R}) \mathrm{e}-\mathrm{R} 1$ & 93B6-7;93D2 & $\mathrm{L}$ \\
\hline 241 & 2586 & Df(3R)23D1 & 94A3-4;94D1-4 & 77.56 \\
\hline 242 & 8491 & Df(3R)BSC55 & 94D2-10;94E1-6 & 56.48 \\
\hline 243 & 8583 & Df(3R)BSC56 & 94E1-2;94F1-2 & 78.45 \\
\hline 244 & 7990 & Df(3R)Exel9012 & 94E9;94E13 & $126 * *$ \\
\hline 245 & 9497 & Df(3R)BSC137 & 94F1;95A4 & $48.79 *$ \\
\hline 246 & 7674 & Df(3R)Exel6195 & 95A4;95B1 & 74.66 \\
\hline 247 & 4940 & $\operatorname{Df}(3 R) m b c-30$ & 95A5-7;95C10-11 & $117.64 * *$ \\
\hline 248 & 2585 & $\mathrm{Df}(3 \mathrm{R}) \mathrm{mbc}-\mathrm{R} 1$ & 95A5-7;95D6-11 & $133.74 * *$ \\
\hline 249 & 7992 & Df(3R)Exel9014 & 95B1;95D1 & 95.60 \\
\hline
\end{tabular}


APPENDIX RESULTS

\begin{tabular}{|c|c|c|c|c|}
\hline 250 & 7675 & Df(3R)Exel6196 & 95C12;95D8 & $108.21 * *$ \\
\hline 251 & 4432 & $\operatorname{Df}(3 R)$ crb-F89-4 & 95D7-D11;95F15 & 44.54 \\
\hline 252 & 7676 & Df(3R)Exel6197 & 95D8;95E1 & $135.12 * *$ \\
\hline 253 & 2363 & Df(3R)crb87-5 & 95F7;96A17-18 & 65.89 \\
\hline 254 & 3468 & $\operatorname{Df}(3 \mathrm{R}) \operatorname{slo} 8$ & 96A2-7;96D2-4 & 88.61 \\
\hline 255 & 7681 & Df(3R)Exel6202 & 96D1;96D1 & $173.8^{* *}$ \\
\hline 256 & 7682 & Df(3R)Exel6203 & 96Е2;96Е6 & $141.1^{* *}$ \\
\hline 257 & 9500 & Df(3R)BSC140 & 96F1;96F10 & 96.31 \\
\hline 258 & 5601 & Df(3R)Espl3 & 96F1;97B1 & 61.64 \\
\hline 259 & 1910 & Df(3R)Tl-P & 97A;98A1-2 & 49.14 \\
\hline 260 & 823 & Df(3R)D605 & 97E3;98A5 & 80.34 \\
\hline 261 & 9529 & Df(3R)IR16 & 97F1-2;98A & $172.1 * *$ \\
\hline 262 & 7412 & $\mathrm{Df}(3 \mathrm{R}) \mathrm{BSC} 42$ & 98B1-2;98B3-5 & $118.53^{* * *}$ \\
\hline 263 & 430 & $\mathrm{Df}(3 \mathrm{R}) 3450$ & 98E3;99A6-8 & $143.77 * *$ \\
\hline 264 & 669 & Df(3R)Dr-rv1 & 99A1-2;99B6-11 & $134.41 * *$ \\
\hline 265 & 3547 & Df(3R)L127 & 99B5-6;99F1 & 91.08 \\
\hline 266 & 3546 & $\mathrm{Df}(3 \mathrm{R}) \mathrm{B} 81$ & 99D3;3Rt & 77.99 \\
\hline 267 & 1785 & $\mathrm{C}(4) \mathrm{RM}$ & 101F1;102F8 & $33.43 *$ \\
\hline 268 & 8067 & Df(4)ED6366 & 102A1;102A6 & SL \\
\hline 269 & 7084 & $\mathrm{Df}(4) \mathrm{O} 2$ & $102 \mathrm{C} 02 ; 102 \mathrm{D} 02$ & 96.43 \\
\hline 270 & 759 & $\mathrm{Df}(4) \mathrm{G}$ & 102E2-7;102E-F2 & $118.11 * *$ \\
\hline
\end{tabular}

Table A2-2. Breeding Crosses to Investigate Deficiency X ddc $>\alpha$-Synuclein[A53T] Lethality.

\begin{tabular}{|l|l|l|l|l|l|l|}
\hline $\begin{array}{l}\text { GENE } \\
\text { LOCATION }\end{array}$ & $\begin{array}{l}\text { GENE } \\
\text { NUMBER/ }\end{array}$ & $\begin{array}{l}\text { CG } \\
\text { NAME }\end{array}$ & $\begin{array}{l}\text { Vienna } \\
\text { NUAB }\end{array}$ & \multicolumn{1}{l|}{$\begin{array}{l}\text { BL } \\
\text { Stock }\end{array}$} & Lethal? \\
\hline $\begin{array}{l}\text { Lethal cross: deficiency BL 5281. 5C3-10;6C3-12 } \\
\text { Identified lethal: kdn (BL 14436) }\end{array}$ & $\begin{array}{l}|l| \\
\text { 5C2-5C3 }\end{array}$ & CG15765 & CG15765 & & 18597 & \\
\hline 5C3-5C3 & CG11462 & CG11462 & & & \\
\hline 5C3-5C3 & CG12729 & CG12729 & & & \\
\hline 5C5-5C5 & CG15764 & CG15764 & & & \\
\hline 5C5-5C5 & CG3033 & CG3033 & 7086 & & \\
\hline 5C5-5C5 & mof & CG3025 & & & \\
\hline 5C5-5C6 & CG3016 & CG3016 & 7090 & & \\
\hline 5C6-5C6 & CG16721 & CG16721 & & & \\
\hline 5C7-5C7 & Act5C & CG4027 & 7139 & & \\
\hline 5C7-5C7 & CG4020 & CG4020 & & 17170 & \\
\hline 5C7-5C7 & CG12236 & CG12236 & & & \\
\hline 5C7-5C7 & CG3011 & CG3011 & 19208 & & \\
\hline 5C7-5C10 & CG3726 & CG3726 & & 19187 & \\
\hline 5C10-5C10 & CG12728 & CG12728 & & & \\
\hline 5C10-5C10 & CG32756 & CG32756 & & & \\
\hline
\end{tabular}




\section{APPENDIX RESULTS}

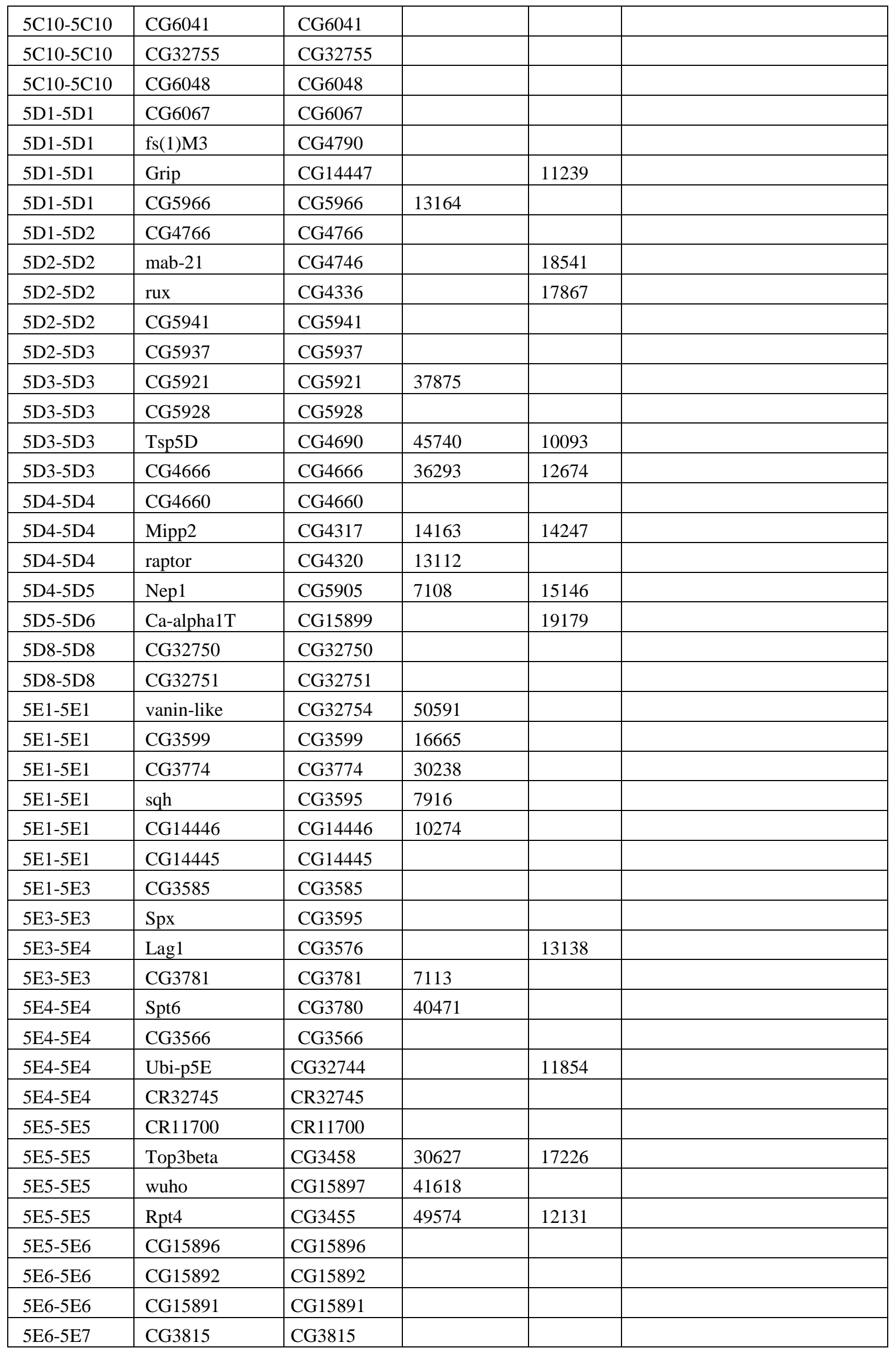




\section{APPENDIX RESULTS}

\begin{tabular}{|c|c|c|c|c|c|}
\hline $5 \mathrm{E} 7-5 \mathrm{E} 7$ & CG12219 & CG12219 & & & \\
\hline $5 \mathrm{E} 7-5 \mathrm{E} 8$ & CG3823 & CG3823 & & & \\
\hline $5 \mathrm{~F} 1-5 \mathrm{~F} 1$ & CG15894 & CG15894 & & 18304 & \\
\hline $5 \mathrm{~F} 2-5 \mathrm{~F} 2$ & CG3842 & CG3842 & 7117 & 19166 & \\
\hline $5 \mathrm{~F} 2-5 \mathrm{~F} 2$ & CG3847 & CG3847 & & & \\
\hline $5 \mathrm{~F} 2-5 \mathrm{~F} 2$ & CG3446 & CG3446 & 42696 & & \\
\hline $5 \mathrm{~F} 2-5 \mathrm{~F} 4$ & $\mathrm{kdn}$ & CG3861 & $\begin{array}{l}26301 \text { (SL } \\
\text { with elav, no } \\
\text { effect on } \\
\text { viability with } \\
\text { ddc) }\end{array}$ & 14436 & lethal \\
\hline $5 \mathrm{~F} 4-5 \mathrm{~F} 4$ & swa & CR32747 & & 18431 & \\
\hline $5 \mathrm{~F} 4-5 \mathrm{~F} 4$ & Marf & CG3429 & & & \\
\hline $5 \mathrm{~F} 4-5 \mathrm{~F} 4$ & $\mathrm{PpV}$ & CG12217 & 31690 & & \\
\hline 5F5-5F5 & CG33664 & CG33664 & & & \\
\hline $5 \mathrm{~F} 6-5 \mathrm{~F} 6$ & CG33669 & CG33669 & & & \\
\hline 6A1-6A1 & CG33668 & CG33668 & & & \\
\hline $6 A 2-6 A 2$ & CG3367 & CG3367 & & 18325 & \\
\hline $6 \mathrm{~A} 2-6 \mathrm{~A} 2$ & CG33667 & CG33667 & & & \\
\hline $6 \mathrm{~A} 2-6 \mathrm{~A} 2$ & CG33666 & CG33666 & & & \\
\hline $6 A 2-6 A 2$ & CG33665 & CG33665 & 51606 & & \\
\hline $6 \mathrm{~A} 3-6 \mathrm{~A} 3$ & CG12543 & CG12543 & & & \\
\hline $6 \mathrm{~B} 1-6 \mathrm{~B} 1$ & $\begin{array}{l}\text { tRNA:CR32748- } \\
\text { RA }\end{array}$ & & & & \\
\hline 6B1-6B1 & tRNA:CR32748 & & & & \\
\hline 6B1-6B1 & CG3918 & CG3918 & & 19185 & \\
\hline 6B1-6B1 & CG3342 & CG3342 & & 18549 & \\
\hline 6B1-6B1 & Spat & CG3926 & & & \\
\hline 6B1-6B1 & RpL7A & CG3314 & 43760 & 18043 & \\
\hline $6 \mathrm{~B} 1-6 \mathrm{~B} 1$ & $\begin{array}{l}\text { snoRNA:Or- } \\
\text { CD10-RA }\end{array}$ & & & & \\
\hline $6 \mathrm{~B} 1-6 \mathrm{~B} 1$ & $\begin{array}{l}\text { snoRNA:Or- } \\
\text { CD10 }\end{array}$ & & & & \\
\hline 6B1-6B2 & $\mathrm{dx}$ & CG3929 & 7795 & & \\
\hline $6 B 2-6 B 3$ & CG34417 & CG34417 & & 13234 & \\
\hline $6 \mathrm{C} 1-6 \mathrm{C} 1$ & CG17717 & CG17717 & & & \\
\hline $6 \mathrm{C} 1-6 \mathrm{C} 1$ & Pat1 & CG10695 & 27307 & 18059 & \\
\hline $6 \mathrm{C} 1-6 \mathrm{C} 1$ & APC7 & CG14444 & 17261 & 15885 & \\
\hline $6 \mathrm{C} 1-6 \mathrm{C} 3$ & CG3973 & CG3973 & 34772 & 12446 & \\
\hline $6 \mathrm{C} 2-6 \mathrm{C} 2$ & CG14443 & CG14443 & & & \\
\hline $6 \mathrm{C} 3-6 \mathrm{C} 3$ & CG3226 & CG3226 & 47034 & & \\
\hline $6 \mathrm{C} 3-6 \mathrm{C} 3$ & 1(1)G0148 & CG32742 & & & \\
\hline $6 \mathrm{C} 3-6 \mathrm{C} 4$ & CG3224 & CG3224 & 29561 & 11243 & \\
\hline $6 \mathrm{C} 4-6 \mathrm{C} 4$ & $\mathrm{Ctr} 1 \mathrm{~A}$ & CG3977 & 46757 & 11243 & \\
\hline $6 \mathrm{C} 4-6 \mathrm{C} 4$ & CG3198 & CG3198 & & 15397 & \\
\hline $6 \mathrm{C} 4-6 \mathrm{C} 5$ & Mcm6 & CG4039 & 13661 & & \\
\hline $6 C 5-6 C 5$ & CG3192 & CG3192 & 30413 & & \\
\hline
\end{tabular}




\section{APPENDIX RESULTS}

\begin{tabular}{|c|c|c|c|c|c|}
\hline $6 \mathrm{C} 5-6 \mathrm{C} 5$ & l(1)G0255 & CG4094 & 34797 & & \\
\hline $6 \mathrm{C} 5-6 \mathrm{C} 5$ & CG4095 & CG4095 & 47685 & & \\
\hline 6С6-6С6 & Pink1 & CG4523 & 21860 & & \\
\hline $6 \mathrm{C} 6-6 \mathrm{C} 6$ & CG3184 & CG3184 & 25880 & & \\
\hline $6 \mathrm{C} 6-6 \mathrm{C} 7$ & CG14442 & CG14442 & & 18974 & \\
\hline $6 \mathrm{C} 7-6 \mathrm{C} 7$ & CG32741 & CG32741 & & & \\
\hline $6 \mathrm{C} 7-6 \mathrm{C} 7$ & CG14440 & CG14440 & 44474 & 12468 & \\
\hline 6C9-6C9 & CG14441 & CG14441 & & 19335 & \\
\hline $6 \mathrm{C} 9-6 \mathrm{C} 10$ & CG3168 & CG3168 & 48010 & 12731 & \\
\hline $6 \mathrm{C} 10-6 \mathrm{C} 10$ & RpL17 & CG3203 & 41778 & 10994 & \\
\hline $6 \mathrm{C} 10-6 \mathrm{C} 10$ & $\begin{array}{l}\text { snoRNA:Psi28S } \\
-3436 \mathrm{~b}\end{array}$ & & & & \\
\hline $6 \mathrm{C} 10-6 \mathrm{C} 10$ & \multicolumn{2}{|c|}{ snoRNA:Psi28S-3436b-RA } & & & \\
\hline $6 \mathrm{C} 10-6 \mathrm{C} 10$ & \multicolumn{2}{|c|}{ snoRNA:Psi28S-3436a-RA } & & & \\
\hline $6 \mathrm{C} 10-6 \mathrm{C} 10$ & $\begin{array}{l}\text { snoRNA:Psi28S } \\
-3436 \mathrm{a}\end{array}$ & & & & \\
\hline $6 \mathrm{C} 10-6 \mathrm{C} 10$ & \multicolumn{2}{|c|}{ snoRNA:Psi28S-1060-RA } & & & \\
\hline $6 \mathrm{C} 10-6 \mathrm{C} 10$ & $\begin{array}{l}\text { snoRNA:Psi28S } \\
-1060\end{array}$ & & & & \\
\hline $6 \mathrm{C} 10-6 \mathrm{C} 10$ & $\begin{array}{l}\text { snoRNA:Psi28S } \\
-1232\end{array}$ & & & & \\
\hline $6 \mathrm{C} 10-6 \mathrm{C} 10$ & \multicolumn{2}{|c|}{ snoRNA:Psi28S-1232-RA } & & & \\
\hline $6 \mathrm{C} 10-6 \mathrm{C} 10$ & tRNA:CR32740 & & & & \\
\hline $6 \mathrm{C} 10-6 \mathrm{C} 10$ & $\begin{array}{l}\text { tRNA:CR32740- } \\
\text { RA }\end{array}$ & & & & \\
\hline $6 \mathrm{C} 11-6 \mathrm{C} 12$ & CG14439 & CG14439 & & & \\
\hline $6 \mathrm{C} 12-6 \mathrm{C} 13$ & CG14438 & CG14438 & & 13068 & \\
\hline \multicolumn{6}{|l|}{122 genes } \\
\hline \multicolumn{6}{|c|}{ Lethal Cross: BL 4953. Df(1)BK10. } \\
\hline & & & & & \\
\hline $15 \mathrm{~F} 2-15 \mathrm{~F} 2$ & CG8918 & CG8918 & 28994 & & \\
\hline $15 \mathrm{~F} 3-15 \mathrm{~F} 3$ & CG5070 & CG5070 & 6150 & & \\
\hline $15 \mathrm{~F} 3-15 \mathrm{~F} 3$ & CG32564 & CG32564 & & & \\
\hline $15 \mathrm{~F} 3-15 \mathrm{~F} 3$ & CG32563 & CG32563 & & & \\
\hline $15 \mathrm{~F} 3-15 \mathrm{~F} 3$ & CG12995 & CG12995 & & & \\
\hline $15 \mathrm{~F} 3-15 \mathrm{~F} 4$ & CG18258 & CG18258 & 20621 & & \\
\hline $15 \mathrm{~F} 4-15 \mathrm{~F} 4$ & CG5162 & CG5162 & & & \\
\hline $15 \mathrm{~F} 4-15 \mathrm{~F} 4$ & CG12998 & CG12998 & & & \\
\hline $15 \mathrm{~F} 4-15 \mathrm{~F} 4$ & CG5172 & CG5172 & & & \\
\hline $15 \mathrm{~F} 4-15 \mathrm{~F} 4$ & CG12997 & CG12997 & & & \\
\hline $15 \mathrm{~F} 4-15 \mathrm{~F} 4$ & CG34327 & CG34327 & & & \\
\hline $15 \mathrm{~F} 4-15 \mathrm{~F} 4$ & CG10598 & CG10598 & & & \\
\hline $15 \mathrm{~F} 4-15 \mathrm{~F} 4$ & CG10597 & CG10597 & 6157 & & \\
\hline $15 \mathrm{~F} 4-15 \mathrm{~F} 4$ & CG8915 & CG8915 & 28857 & & \\
\hline $15 \mathrm{~F} 4-15 \mathrm{~F} 4$ & CG8675 & CG8675 & 26997 & & \\
\hline $15 \mathrm{~F} 4-15 \mathrm{~F} 4$ & CG12996 & CG12996 & & & \\
\hline
\end{tabular}




\section{APPENDIX RESULTS}

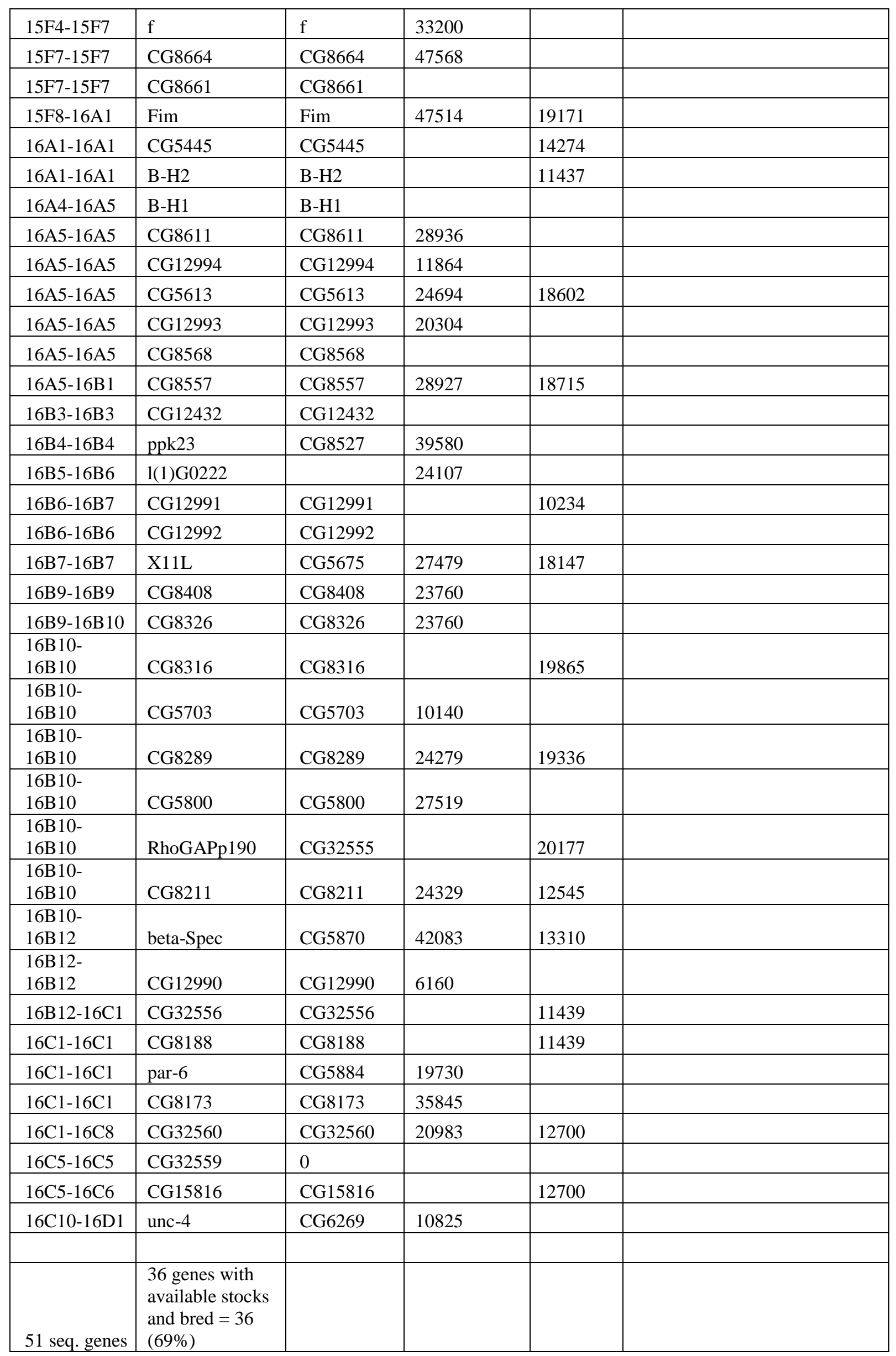




\section{APPENDIX RESULTS}

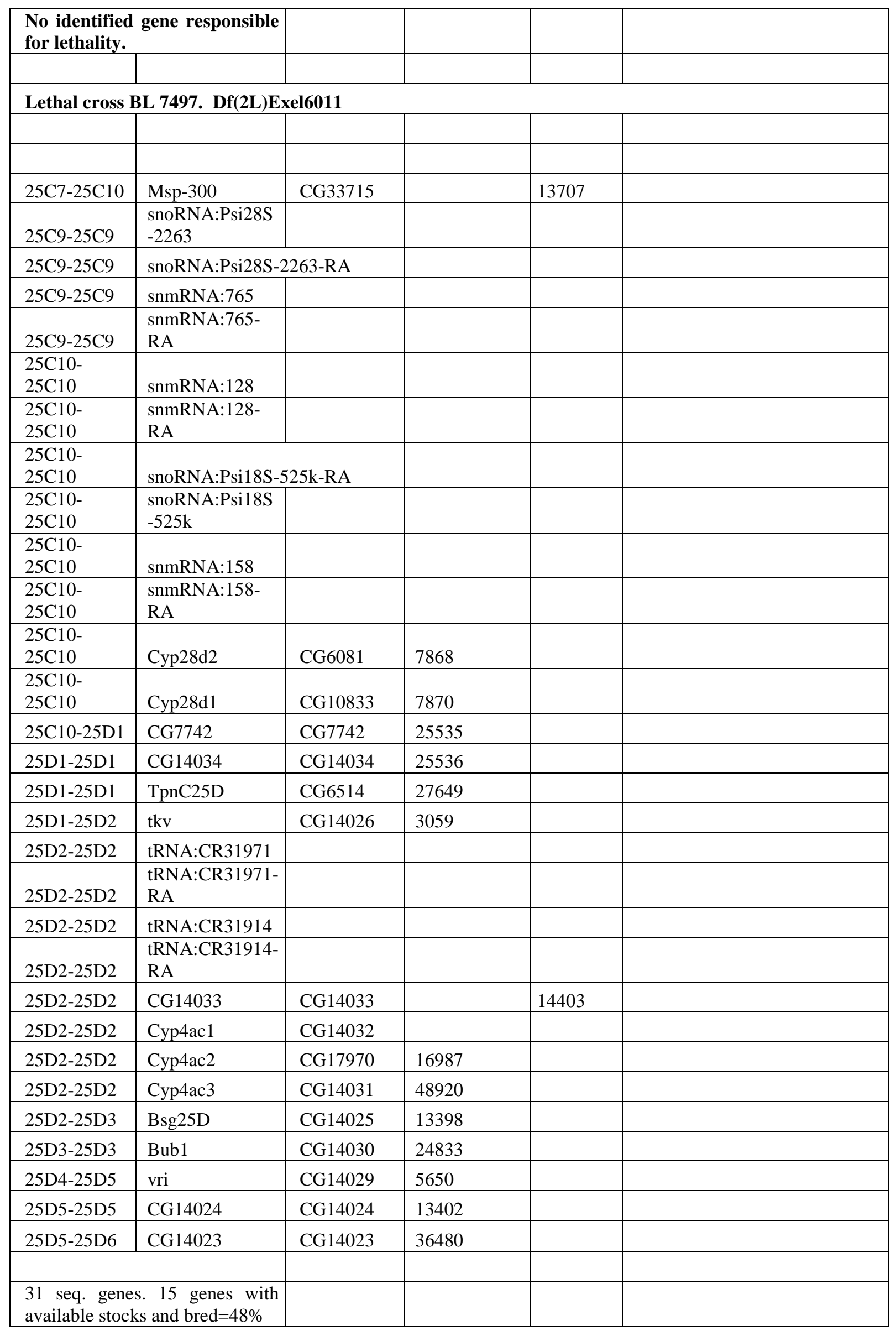




\section{APPENDIX RESULTS}

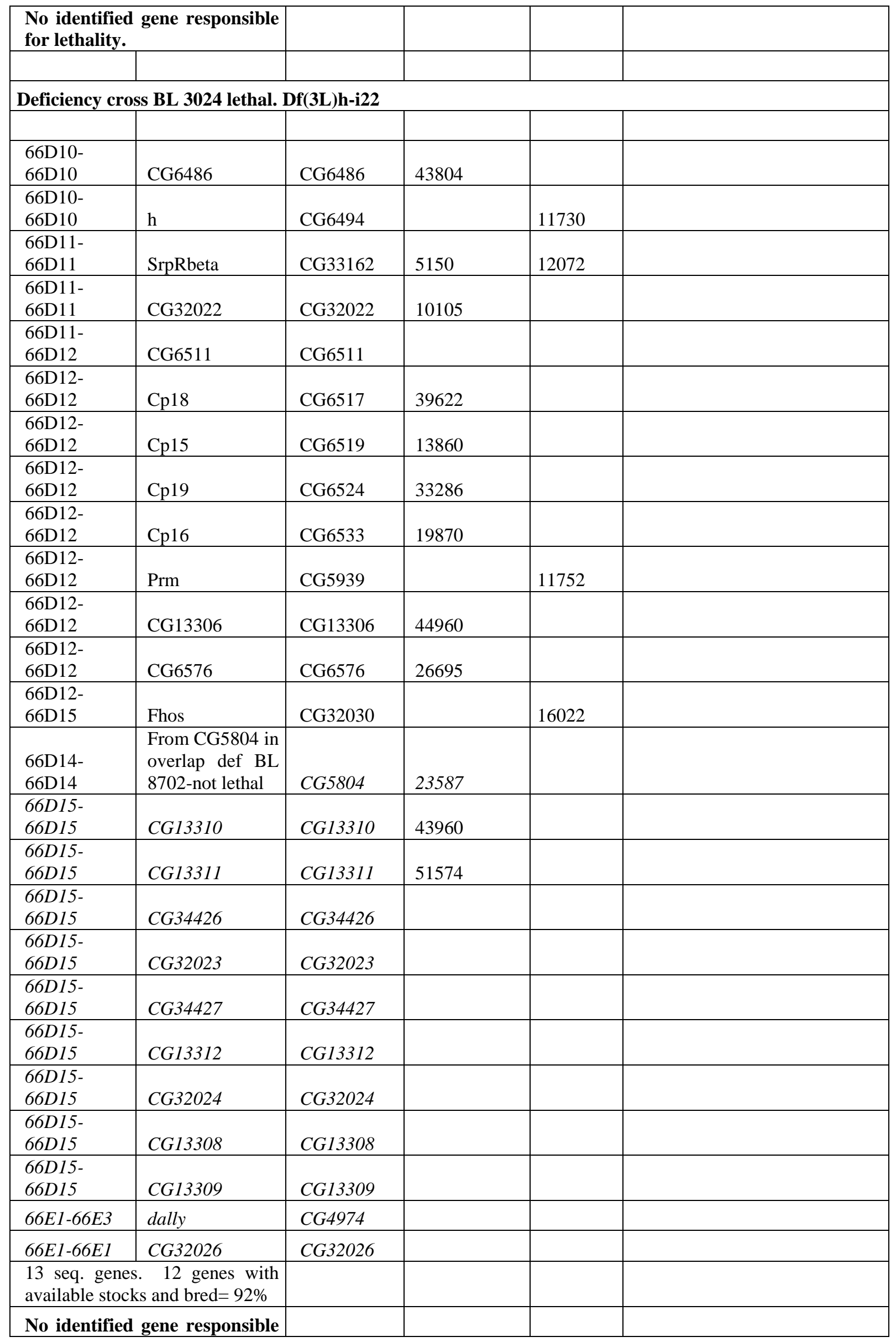




\section{APPENDIX RESULTS}

\begin{tabular}{|c|c|c|c|c|c|}
\hline \multicolumn{2}{|l|}{ for lethality. } & & & & \\
\hline \multicolumn{5}{|c|}{ Deficiency Cross BL 3340 lethal. Df(3R)e-R1 } & \\
\hline & & & & & \\
\hline 93B5-93B7 & Dhc93AB & CG3723 & 41947 & & \\
\hline 93B7-93B7 & CG12278 & CG12278 & 29484 & & \\
\hline 93B7-93B7 & CG31189 & CG31189 & 22962 & & \\
\hline 93B7-93B7 & CG31207 & CG31207 & & & \\
\hline 93B7-93B7 & CG7079 & CG7079 & & & \\
\hline 93B7-93B7 & CG17279 & CG17279 & & & \\
\hline 93B7-93B8 & Mvl & CG3671 & 44000 & 19886 & \\
\hline 93B8-93B9 & Cortactin & CG3637 & & 22381 & \\
\hline 93B9-93B10 & AnnIX & CG5730 & 27493 & & \\
\hline $\begin{array}{l}93 \mathrm{~B} 10- \\
93 \mathrm{~B} 10\end{array}$ & $\mathrm{r}-1$ & CG3593 & 21688 & 17982 & \\
\hline $\begin{array}{l}\text { 93B10- } \\
93 \mathrm{~B} 10\end{array}$ & dmrt93B & CG5737 & 41048 & & \\
\hline $\begin{array}{l}\text { 93B10- } \\
93 \mathrm{~B} 10\end{array}$ & CG7056 & CG7056 & & 17943 & \\
\hline $\begin{array}{l}\text { 93B10- } \\
\text { 93B11 }\end{array}$ & RhoGAP93B & CG3421 & 41934 & 19720 & \\
\hline $\begin{array}{l}\text { 93B11- } \\
93 \mathrm{~B} 12\end{array}$ & CG7044 & CG7044 & 27811 & 12413 & \\
\hline $\begin{array}{l}93 \mathrm{~B} 12- \\
93 \mathrm{~B} 12\end{array}$ & CG5745 & CG5745 & 35034 & & \\
\hline $\begin{array}{l}\text { 93B12- } \\
93 \mathrm{~B} 12\end{array}$ & $\sec 15$ & CG7034 & & & \\
\hline $\begin{array}{l}\text { 93B12- } \\
\text { 93B12 }\end{array}$ & rtet & CG5760 & 44002 & 11714 & \\
\hline $\begin{array}{l}\text { 93B12- } \\
93 \mathrm{~B} 13\end{array}$ & Rab11 & CG5771 & 22198 & 12418 & \\
\hline $\begin{array}{l}\text { 93B13- } \\
93 \mathrm{~B} 13\end{array}$ & ppan & CG5786 & 39001 & 11557 & \\
\hline $\begin{array}{l}\text { 93B13- } \\
93 \mathrm{~B} 13\end{array}$ & CG17282 & CG17282 & & & \\
\hline 93B13-93C1 & slmb & CG3412 & & 11493 & \\
\hline $93 \mathrm{C} 1-93 \mathrm{C} 1$ & CG5793 & CG5793 & 47883 & 16067 & \\
\hline $93 \mathrm{C} 1-93 \mathrm{C} 1$ & Obp93a & CG17284 & 2558 & & \\
\hline $93 \mathrm{C} 1-93 \mathrm{C} 1$ & CG10825 & CG10825 & 31360 & & \\
\hline $93 \mathrm{C} 1-93 \mathrm{C} 1$ & CG7009 & CG7009 & 27790 & 18008 & \\
\hline $93 \mathrm{C} 1-93 \mathrm{C} 1$ & CG5798 & CG5798 & 8931 & & \\
\hline $93 \mathrm{C} 1-93 \mathrm{C} 1$ & CG5802 & CG5802 & 6801 & 13460 & \\
\hline $93 \mathrm{C} 1-93 \mathrm{C} 5$ & SNF4Agamma & CG17299 & & 13088 & \\
\hline $93 \mathrm{C} 2-93 \mathrm{C} 2$ & CG10824 & \begin{tabular}{|l|} 
CG10824 \\
\end{tabular} & 16588 & & \\
\hline $93 \mathrm{C} 2-93 \mathrm{C} 2$ & CG5810 & CG5810 & 44988 & & \\
\hline $93 \mathrm{C} 2-93 \mathrm{C} 2$ & CG7000 & CG7000 & 42496 & & \\
\hline 93C6-93C6 & CG5862 & CG5862 & 45155 & & \\
\hline 93C6-93C6 & CG3353 & CG3353 & 41920 & 7353 & \\
\hline 93C6-93C7 & CG5871 & CG5871 & 41822 & 13618 & \\
\hline $93 \mathrm{C7}-93 \mathrm{C7}$ & CG3337 & CG3337 & 29170 & & \\
\hline
\end{tabular}




\section{APPENDIX RESULTS}

\begin{tabular}{|l|l|l|l|l|l|}
\hline 93C7-93C7 & NELF-A & CG5874 & 43211 & 15189 & \\
\hline 93C7-93D1 & e & CG3331 & 45689 & & \\
\hline 93D1-93D1 & CG5892 & CG5892 & 6807 & 18496 & \\
\hline 93D1-93D2 & ETHR & CG5911 & 42716 & & \\
\hline 93D2-93D2 & Rab1 & CG3320 & & 17936 & \\
\hline 93D2-93D2 & AP-2sigma & CG6056 & 34148 & 13478 & \\
\hline 93D2-93D2 & CG5919 & CG5919 & 28402 & & \\
\hline 93D2-93D2 & CG3308 & CG3308 & 43756 & & \\
\hline 93D2-93D2 & CG3301 & CG3301 & 43255 & 18137 & \\
\hline 93D2-93D4 & SIFR & CG10823 & 1783 & 16202 & \\
\hline $\begin{array}{l}\text { 93D3- } \\
\text { 93D3 }\end{array}$ & CG17298 & CG17298 & & & \\
\hline $\begin{array}{l}\text { 93D4- } \\
\text { 93D4 }\end{array}$ & Hsromega-RB & & & & \\
\hline $\begin{array}{l}\text { 93D4- } \\
\text { 93D4 }\end{array}$ & Hsromega & & & & \\
\hline $\begin{array}{l}\text { 93D4- } \\
\text { 93D4 }\end{array}$ & Hsromega-RA & & & & \\
\hline $\begin{array}{l}\text { 93D4- } \\
\text { 93D4 }\end{array}$ & Hsromega-RC & & & & \\
\hline $\begin{array}{l}\text { 51 seq. } \\
\text { available and bred=76\% }\end{array}$ & & & & \\
\hline $\begin{array}{l}\text { No identified gene responsible } \\
\text { for lethality. }\end{array}$ & & & & \\
\hline \multicolumn{2}{|l|}{} & & & & \\
\hline
\end{tabular}




\section{APPENDIX RESULTS}

Table A2-3. Preliminary data from secondary screen: Genes screened for effect on DA, longevity and oxidative stress sensitivity when in combination with ddc $>\alpha$ Synuclein[A53T]. In some cases, flies also tested with elav>A53T/+ (if tested, data also shown). Control in this case was comparison to either ddc $>$ A53T/+ or elav $>$ A53T/+. Low DA HPLC results for $\mathrm{ddc}>\mathrm{A} 53 \mathrm{~T}$ are mean values. $\mathrm{Nt}=$ not tested. A minimum of 20 flies were used for oxidative stress and longevity tests. Information on genes based on listed functions from Flybase and gene categorization from Osprey Network Visualization software (1.2.0, made by Samuel Lunenfeld Research Institute, Toronto, Canada). http://flybase.org/ http://biodata.mshri.on.ca/osprey/servlet/Index

\begin{tabular}{|c|c|c|c|c|c|c|c|c|c|}
\hline Gene & Protein Name & Function & $\begin{array}{l}\text { Gene } \\
\text { Category }\end{array}$ & $\begin{array}{l}\text { Human } \\
\text { Homologue }\end{array}$ & Stocks Used & DA Content & $\begin{array}{l}\text { Sensitivity to H202 } \\
\text { (median survival } \\
\text { for lines showing } \\
\text { differences) } \\
\text { Ddc }>[\text { A53T }] /+=6 \mathbf{d} \\
\text { Elav }>[\text { A53T }] /+=5 d\end{array}$ & $\begin{array}{l}\text { Sensitivity to } \\
\text { Paraquat } \\
\text { (median survival } \\
\text { for lines showing } \\
\text { differences) } \\
\text { Ddc }>[\text { A53T] } /+=40 \\
\text { hr } \\
\text { Elav }>[\text { A53T] } /+=40 \\
\text { hr }\end{array}$ & $\begin{array}{l}\text { Alteration in } \\
\text { Longevity (median } \\
\text { survival in days for } \\
\text { lines showing } \\
\text { differences) } \\
\text { Ddc }>[\text { A53T] }]+=56 \mathrm{~d}\end{array}$ \\
\hline \multicolumn{10}{|c|}{$\begin{array}{l}\text { In ID Def } \\
\text { and Chen } e t \\
\text { al: }\end{array}$} \\
\hline \multirow[t]{2}{*}{ CG1906 } & \multirow[t]{2}{*}{ alphabet/PP2c } & \multirow{2}{*}{$\begin{array}{l}\text { protein } \\
\text { serine/threonine } \\
\text { phosphatise; negative } \\
\text { regulator Ras and } \\
\text { MAPK signal } \\
\text { transduction }\end{array}$} & \multirow{2}{*}{$\begin{array}{l}\text { Signal } \\
\text { transduction; } \\
\text { Metabolism; } \\
\text { protein amino acid } \\
\text { dephosphorylation }\end{array}$} & \multirow[t]{2}{*}{$\begin{array}{l}\text { PPM1B-protein phosphatase, } \\
\text { Mg2+/Mn2+ dependent, 1B }\end{array}$} & BL 23341 & $\begin{array}{l}\text { Ddc:ns } \\
\text { Elav: ns }\end{array}$ & Ddc: ns Elav:ns & Ddc: ns Elav:ns & ns \\
\hline & & & & & VD32476 & $\begin{array}{l}\text { Ddc:69.86 } \\
\text { Elav:16.15 }\end{array}$ & Ddc: ns Elav: ns & Ddc: ns Elav:ns & $\mathrm{ns}$ \\
\hline CG2038 & CSN7 & $\begin{array}{l}\text { protein stability and } \\
\text { proteolysis }\end{array}$ & Unknown & $\begin{array}{l}\text { COPS7A-COP9 constitutive } \\
\text { photomorphogenic homolog } \\
\text { subunit 7A }\end{array}$ & BL 23376 & ns & & & \\
\hline CG2713 & Tiny tim 50 & Mt translocator & Metabolism & $\begin{array}{l}\text { TIMM50-translocase of inner } \\
\text { mitochondrial membrane } 50 \\
\text { homolog }\end{array}$ & VD5587 & 41.0 & $\begin{array}{l}\text { increased } \\
\text { sensitivity (3d) }\end{array}$ & $\mathrm{ns}$ & 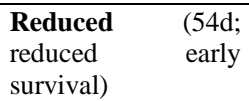 \\
\hline CG3572 & vimar & $\begin{array}{l}\text { signalling, small } \\
\text { GTPase mediated } \\
\text { signalling }\end{array}$ & $\begin{array}{l}\text { Signal } \\
\text { Transduction }\end{array}$ & $\begin{array}{l}\text { RAP1GDS1-RAP1, GTP- } \\
\text { GDP dissociation stimulator } \\
1\end{array}$ & BL11199 & 132.0 & $\mathrm{nt}$ & $\mathrm{nt}$ & $\mathrm{nt}$ \\
\hline CG3612 & bellweather & $\begin{array}{l}\text { mitochondrial proton } \\
\text { transporting ATP } \\
\text { synthase complex, } \\
\text { catalytic core } \mathrm{F}(1)- \\
\text { ATP biosynthetic } \\
\text { process }\end{array}$ & $\begin{array}{l}\text { Cell organization; } \\
\text { metabolism; } \\
\text { transport }\end{array}$ & $\begin{array}{l}\text { ATP5A1- ATP synthase, H+ } \\
\text { transporting, mitochondrial } \\
\text { F1 complex, alpha subunit } 1\end{array}$ & BL 19869 & 46.9 & $\mathrm{~ns}$ & $\begin{array}{l}\text { Ddc: ns } \\
\text { Elav: increased } \\
\text { sensitivity (22h) }\end{array}$ & $\mathrm{ns}$ \\
\hline CG4119 & & $\begin{array}{l}\text { Nuclear mRNA } \\
\text { splicing via } \\
\text { spliceosome }\end{array}$ & RNA processing & $\begin{array}{l}\text { RBM } 25 \text { - RNA binding motif } \\
\text { protein } 25\end{array}$ & VD 26395 & ns & nt & $\mathrm{nt}$ & nt \\
\hline
\end{tabular}




\section{APPENDIX RESULTS}

\begin{tabular}{|c|c|c|c|c|c|c|c|c|c|}
\hline \multirow[t]{2}{*}{ CG4494 } & \multirow[t]{2}{*}{ Smt3 } & \multirow{2}{*}{$\begin{array}{l}\text { protein stability and } \\
\text { proteolysis, } \\
\text { sumolation }\end{array}$} & \multirow{2}{*}{$\begin{array}{l}\text { Protein transport; } \\
\text { metabolism; cell } \\
\text { organization and } \\
\text { biogenesis }\end{array}$} & \multirow{2}{*}{$\begin{array}{l}\text { SUMO4- SMT3 suppressor } \\
\text { of mif two } 3 \text { homolog } 4\end{array}$} & BL10419 & ns & ns & ns & $\mathrm{ns}$ \\
\hline & & & & & VD34113 & $\begin{array}{l}\text { Semi lethal } \\
\text { with ddc and } \\
\text { elav }\end{array}$ & & & \\
\hline CG7269 & RNA helicase & $\begin{array}{l}\text { ATP-dependent } \\
\text { helicase binding; } \\
\text { RNA helicase } \\
\text { activity; nuclear } \\
\text { mRNA splicing via } \\
\text { spliceosome }\end{array}$ & $\begin{array}{l}\text { Transport; RNA } \\
\text { processing }\end{array}$ & $\begin{array}{l}\text { BAT1- Spliceosome RNA } \\
\text { helicase BAT1 }\end{array}$ & 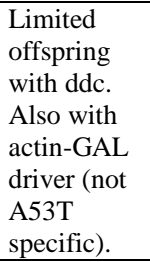 & & & & \\
\hline \multirow[t]{2}{*}{ CG7873 } & \multirow[t]{2}{*}{ Src42a } & \multirow[t]{2}{*}{$\begin{array}{l}\text { ATP binding, protein } \\
\text { tyrosine kinase; } \\
\text { signalling, RTK } \\
\text { pathway, JNK } \\
\text { pathway and EGFR } \\
\text { pathway }\end{array}$} & \multirow[t]{2}{*}{$\begin{array}{l}\text { Cell organization } \\
\text { and biogenesis; } \\
\text { signal } \\
\text { transduction; } \\
\text { protein amino acid } \\
\text { phosphorylation }\end{array}$} & \multirow[t]{2}{*}{ FRK- fyn-related kinase } & VD26019 & 21.4 & $\begin{array}{l}\text { Ddc: increased } \\
\text { sensitivity (5d) } \\
\text { Elav: increased } \\
\text { sensitivity (3d) }\end{array}$ & $\mathrm{ns}$ & $\mathrm{ns}$ \\
\hline & & & & & VD40838 & & $\begin{array}{l}\text { Ddc: increased } \\
\text { sensitivity (5d) }\end{array}$ & & \\
\hline CG8211 & & other & Unknown & $\begin{array}{l}\text { INTS2- integrator complex } \\
\text { subunit } 2\end{array}$ & VD 24237 & ns & & & \\
\hline CG8258 & CCT theta & $\begin{array}{l}\text { Chaperone } \\
\text { containing T } \\
\text { complex; ATP } \\
\text { binding; protein } \\
\text { stability and } \\
\text { proteolysis }\end{array}$ & Metabolism & $\begin{array}{l}\text { CCT8-chaperonin containing } \\
\text { TCP1, subunit } 8 \text { (theta) }\end{array}$ & VD 45789 & $\begin{array}{l}\text { Ddc: } \mathrm{ns} \\
\text { Elav:40.2 }\end{array}$ & $\begin{array}{l}\text { Ddc: ns Elav: } \\
\text { increased } \\
\text { sensitivity (3d) }\end{array}$ & Ddc: ns Elav: ns & $\mathrm{ns}$ \\
\hline CG9911 & & $\begin{array}{l}\text { Protein disulfide } \\
\text { oxidoreductase } \\
\text { activity; electron } \\
\text { transporter activity; } \\
\text { protein modification } \\
\text { process; ER } \\
\end{array}$ & Metabolism & ERP44-ER protein 44 & VD46585 & Ddc:130.0 & $\mathrm{nt}$ & nt & nt \\
\hline CG9932 & & $\begin{array}{l}\text { Proteolysis, } \\
\text { metallopeptidase } \\
\text { activity; zinc ion } \\
\text { binding; nucleus }\end{array}$ & $\begin{array}{l}\text { Protein } \\
\text { Degradation }\end{array}$ & No homologue & $\begin{array}{l}\text { No stock } \\
\text { available at } \\
\text { time } \\
\text { screening }\end{array}$ & & & & \\
\hline CG10230 & Rpn9 & $\begin{array}{l}\text { Proteolysis; } \\
\text { endopeptidase } \\
\text { activity; cytosol }\end{array}$ & $\begin{array}{l}\text { Protein } \\
\text { Degradation }\end{array}$ & $\begin{array}{l}\text { PSMD13-proteasome } \\
\text { (prosome, macropain) 26S } \\
\text { subunit, non-ATPase, } 13\end{array}$ & BL15642 & $\mathrm{ns}$ & $\mathrm{nt}$ & $\mathrm{Nt}$ & nt \\
\hline CG10539 & p70 s6k & $\begin{array}{l}\text { ATP binding; Protein } \\
\text { serine/threonine }\end{array}$ & $\begin{array}{l}\text { Autophagy; Cell } \\
\text { organization and }\end{array}$ & RPS6KB1 & $\begin{array}{l}\text { BL6913 } \\
\text { (UAS-S6k) }\end{array}$ & 40.5 & $\begin{array}{l}\text { increased } \\
\text { sensitivity (4d) }\end{array}$ & $\begin{array}{l}\text { decreased sensitivity } \\
\text { (ns) }\end{array}$ & Ns \\
\hline
\end{tabular}




\section{APPENDIX RESULTS}

\begin{tabular}{|c|c|c|c|c|c|c|c|c|c|}
\hline & & $\begin{array}{l}\text { kinase; signalling, } \\
\text { mTOR, PI3K signal } \\
\text { pathway; ribosome }\end{array}$ & $\begin{array}{l}\text { biogenesis; Cell } \\
\text { Cycle; Signal } \\
\text { Transduction; } \\
\text { protein amino acid } \\
\text { phosphorylation }\end{array}$ & $\begin{array}{l}\text { ribosomal protein S6 kinase, } \\
70 \mathrm{kDa} \text {, polypeptide } 1\end{array}$ & VD18126 & 202.0 & $\begin{array}{l}\text { decreased } \\
\text { sensitivity (9d) }\end{array}$ & $\begin{array}{l}\text { increased sensitivity } \\
\text { (ns) }\end{array}$ & Ns \\
\hline CG10640 & Uev1A & $\begin{array}{l}\text { Ubiquitin-protein } \\
\text { ligase activity; } \\
\text { ubiquitin conjugating } \\
\text { activity }\end{array}$ & $\begin{array}{l}\text { Metabolism; } \\
\text { protein } \\
\text { degradation }\end{array}$ & $\begin{array}{l}\text { UBE2V2-ubiquitin- } \\
\text { conjugating enzyme E2 } \\
\text { variant } 2\end{array}$ & BL12771 & 43.5 & $\mathrm{~ns}$ & $\mathrm{~ns}$ & $\mathrm{~ns}$ \\
\hline CG10938 & ProsMA5 & $\begin{array}{l}\text { 20S proteasome } \\
\text { alpha subunit; } \\
\text { ubiquitin -dependent } \\
\text { protein catabolic } \\
\text { process; threonine } \\
\text { endopeptidase } \\
\text { activity }\end{array}$ & $\begin{array}{l}\text { Protein } \\
\text { degradation }\end{array}$ & $\begin{array}{l}\text { PSMA5-proteasome } \\
\text { (prosome, macropain) } \\
\text { subunit, alpha type, } 5\end{array}$ & VD22556 & $\begin{array}{l}\text { Lethal ddc } \\
\text { and elav. Also } \\
\text { lethal with } \\
\text { driver alone. }\end{array}$ & & & \\
\hline CG12284 & thread & $\begin{array}{l}\text { Drosophila inhibitor } \\
\text { of apoptosis (DIAP); } \\
\text { Ubiquitin ligase } \\
\text { activity }\end{array}$ & $\begin{array}{l}\text { Cell Organization, } \\
\text { Biogenesis; } \\
\text { Metabolism }\end{array}$ & No homologue & BL19865 & 26.7 & $\mathrm{~ns}$ & $\mathrm{~ns}$ & Reduced (44d) \\
\hline CG13133 & & unknown & Unknown & No homologue & BL 17746 & 48.0 & ns & ns & ns \\
\hline$\overline{C G 18176}$ & deflated & unknown & Unknown & $\begin{array}{l}\text { INTS7- integrator complex } \\
\text { subunit } 7\end{array}$ & VD 20604 & 35.0 & $\begin{array}{l}\text { increased } \\
\text { sensitivity }(5 d)\end{array}$ & ns & extended (65d) \\
\hline CG18802 & alpha-Man-II & $\begin{array}{l}\text { Golgi membrane/ER; } \\
\text { carbohydrate } \\
\text { metabolism; protein } \\
\text { amino acid N-linked } \\
\text { glycosylation }\end{array}$ & $\begin{array}{l}\text { Metabolism; } \\
\text { carbohydrate } \\
\text { metabolism }\end{array}$ & $\begin{array}{l}\text { MAN2A1-mannosidase, } \\
\text { alpha, class } 2 \mathrm{~A}, \text { member } 1\end{array}$ & $\begin{array}{l}\text { No stock } \\
\text { available at } \\
\text { time } \\
\text { screening }\end{array}$ & & & & \\
\hline CG5165 & PGM & $\begin{array}{l}\text { phosphoglycerate } \\
\text { mutase activity; } \\
\text { monosaccharide } \\
\text { metabolic process }\end{array}$ & $\begin{array}{l}\text { Metabolism; } \\
\text { carbohydrate } \\
\text { metabolism }\end{array}$ & $\begin{array}{l}\text { PGM1-phosphoglucomutase } \\
1\end{array}$ & VD34953 & $\begin{array}{l}\text { Ddc:32 Elav: } \\
45\end{array}$ & Ddc:ns Elav:ns & $\begin{array}{l}\text { Ddc: increased } \\
\text { sensitivity (36hr) } \\
\text { Elav: increased } \\
\text { sensitivity(35hr) }\end{array}$ & $\mathrm{ns}$ \\
\hline $\begin{array}{l}\text { Further } \\
\text { genes of } \\
\text { interest in } \\
\text { Chen et al: }\end{array}$ & & & & & & & & & \\
\hline
\end{tabular}




\section{APPENDIX RESULTS}

\begin{tabular}{|c|c|c|c|c|c|c|c|c|c|}
\hline CG6593 & PP1alpha & $\begin{array}{l}\text { Protein serine/ } \\
\text { threonine phosphatise } \\
\text { type I complex; } \\
\text { Manganese ion } \\
\text { binding }\end{array}$ & $\begin{array}{l}\text { Protein amino acid } \\
\text { dephosphorylation }\end{array}$ & $\begin{array}{l}\text { PPP1CC/PP1G- protein } \\
\text { phosphatase } 1 \text {, catalytic } \\
\text { subunit, gamma isozyme }\end{array}$ & BL23698 & 55 & ns & ns & Reduced (48d) \\
\hline CG8068 & Suvar & $\begin{array}{l}\text { Negative regulator } \\
\text { JAK-STAT cascade; } \\
\text { regulation of } \\
\text { transcription; } \\
\text { regulation protein } \\
\text { catabolism; SUMO } \\
\text { regulator, nucleus, } \\
\text { cytoplasm }\end{array}$ & $\begin{array}{l}\text { Cell organization } \\
\text { and biogenesis; } \\
\text { metabolism }\end{array}$ & $\begin{array}{l}\text { PIAS1-protein inhibitor of } \\
\text { activated STAT, } 1\end{array}$ & VD30709 & ns & $\begin{array}{l}\text { increased } \\
\text { sensitivity }(4.5 d)\end{array}$ & ns & Ns \\
\hline CG10726 & $\begin{array}{l}\text { Barren } \\
\text { (screened in } \\
\text { error)(not in low } \\
\text { def) }\end{array}$ & $\begin{array}{l}\text { cell cycle regulation, } \\
\text { mitotic chromosome } \\
\text { condensation; PNS } \\
\text { nervous development } \\
\text {; chromosome } \\
\text { condensation }\end{array}$ & $\begin{array}{l}\text { Cell Cycle; Cell } \\
\text { organization and } \\
\text { biogenesis }\end{array}$ & $\begin{array}{l}\text { NCAPH- non-SMC } \\
\text { condensin I complex, subunit } \\
\text { H }\end{array}$ & BL4402 & Ddc:43 & $\begin{array}{l}\text { Ddc: increased } \\
\text { sensitivity (3d) } \\
\text { Elav: increased } \\
\text { sensitivity (3d) }\end{array}$ & Ddc:ns & $\mathrm{Ns}$ \\
\hline \multicolumn{10}{|l|}{$\begin{array}{l}\text { Additional } \\
\text { Genes } \\
\text { Tested: }\end{array}$} \\
\hline \multirow[t]{2}{*}{ CG12276 } & \multirow[t]{2}{*}{ Aos1 } & \multirow{2}{*}{$\begin{array}{l}\text { SUMO activating } \\
\text { enzyme complex; } \\
\text { ubiquitin and SUMO } \\
\text { activating enzyme } \\
\text { activity; nucleus }\end{array}$} & \multirow{2}{*}{$\begin{array}{l}\text { Metabolism; } \\
\text { protein transport; } \\
\text { protein } \\
\text { degradation }\end{array}$} & \multirow[t]{2}{*}{$\begin{array}{l}\text { SAE1-SUMO1 activating } \\
\text { enzyme subunit } 1\end{array}$} & VD47257 & ns & $\begin{array}{l}\text { increased } \\
\text { sensitivity (5d) }\end{array}$ & nt & Ns \\
\hline & & & & & VD18528 & ns & $\mathrm{ns}$ & $\mathrm{nt}$ & extended (67d) \\
\hline CG3018 & lwr & $\begin{array}{l}\text { Ubiquitin-protein } \\
\text { ligase activity; } \\
\text { SUMO conjugating } \\
\text { enzyme activity; } \\
\text { nucleus }\end{array}$ & $\begin{array}{l}\text { Metabolism; } \\
\text { protein transport }\end{array}$ & $\begin{array}{l}\text { UBE21-ubiquitin-conjugating } \\
\text { enzyme E2I }\end{array}$ & VD33684 & $\mathrm{ns}$ & $\mathrm{ns}$ & nt & $\mathrm{Nt}$ \\
\hline CG12359 & Mlp1 & $\begin{array}{l}\text { Ubiquitin-protein } \\
\text { ligase activity; } \\
\text { SUMO conjugating } \\
\text { enzyme activity; } \\
\text { nucleus }\end{array}$ & $\begin{array}{l}\text { Metabolism; } \\
\text { protein transport }\end{array}$ & No homologue & VD31744 & $\mathrm{ns}$ & $\mathrm{nt}$ & nt & $\mathrm{nt}$ \\
\hline CG2151 & Trxr-1 & $\begin{array}{l}\text { thioredoxin reductase } \\
2 \text { isoform } 3\end{array}$ & Metabolism & $\begin{array}{l}\text { TXNRD2-thioredoxin } \\
\text { reductase } 2\end{array}$ & VD47307 & 47.0 & $\mathrm{~ns}$ & $\begin{array}{l}\text { Ddc:ns } \\
\text { Elav: increased } \\
\text { sensitivity (36hr) }\end{array}$ & $\mathrm{ns}$ \\
\hline CG10523 & Parkin & $\begin{array}{l}\text { Ubiquitin E3 ligase; } \\
\text { negative regulator } \\
\text { JNK cascade }\end{array}$ & $\begin{array}{l}\text { Cell organization } \\
\text { and biogenesis; } \\
\text { metabolism; } \\
\text { protein } \\
\text { degradation; DNA } \\
\text { metabolism } \\
\end{array}$ & PARK & BL10006 & Ddc: 36.26 & $\mathrm{nt}$ & nt & $\mathrm{Nt}$ \\
\hline
\end{tabular}




\section{APPENDIX RESULTS}

\begin{tabular}{|c|c|c|c|c|c|c|c|c|c|}
\hline CG4523 & PINK1 & $\begin{array}{l}\text { ATP binding; protein } \\
\text { serine/threonine } \\
\text { kinase; response to } \\
\text { oxidative stress }\end{array}$ & $\begin{array}{l}\text { Cell organization } \\
\text { and biogenesis; } \\
\text { metabolism; } \\
\text { protein amino acid } \\
\text { phosphorylation }\end{array}$ & PINK1 & VD21860 & $\mathrm{ns}$ & $\mathrm{nt}$ & nt & $\mathrm{Nt}$ \\
\hline CG8464 & HTRA2 & $\begin{array}{l}\text { Serine-type } \\
\text { endopeptidase } \\
\text { activity }\end{array}$ & $\begin{array}{l}\text { Protein } \\
\text { degradation }\end{array}$ & HTRA2 & VD24104 & Ddc: 44.0 & $\mathrm{nt}$ & nt & $\mathrm{Nt}$ \\
\hline
\end{tabular}

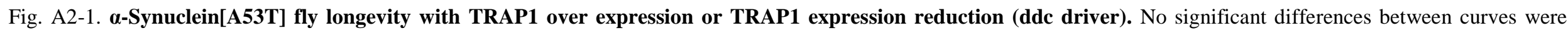
observed. Results using the elav-GAL4 similar (ns) to ddc-GAL4 results. $\mathrm{n}>100$.

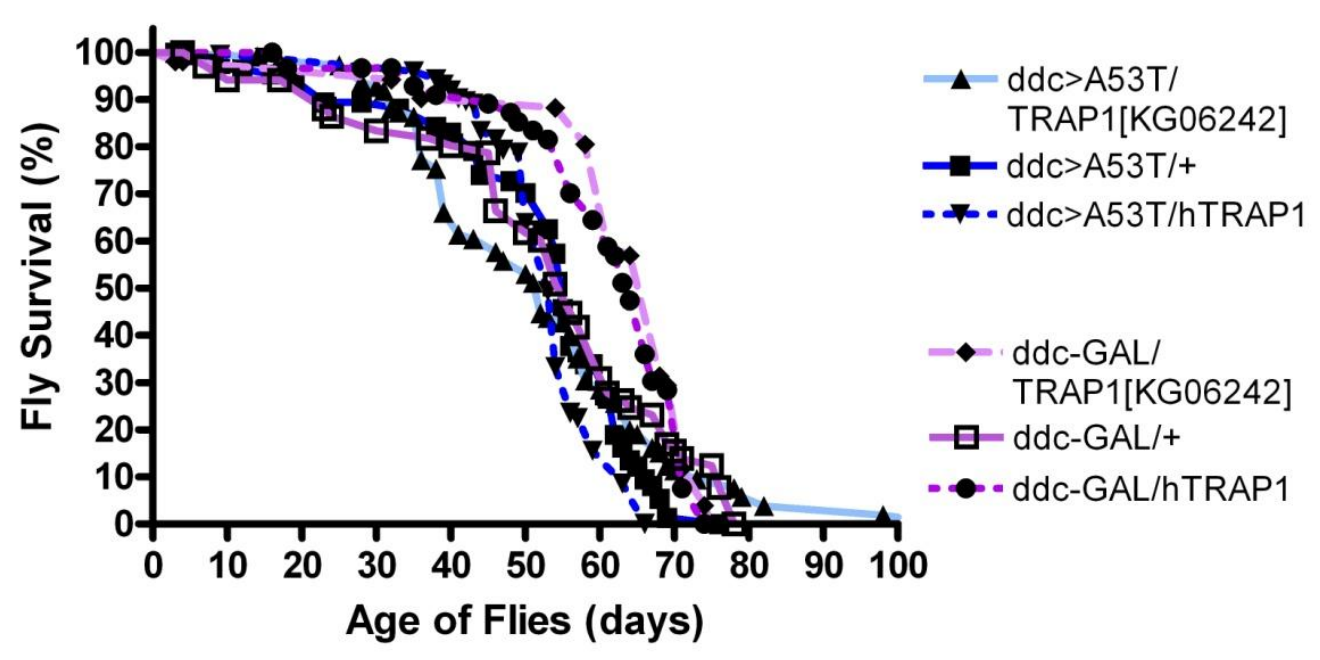


1. Lee FJ, Liu F. Genetic factors involved in the pathogenesis of Parkinson's disease. Brain Res Rev. 2008;58(2):354-364.

2. Mayeux R. Epidemiology of neurodegeneration. Annu Rev Neurosci. 2003;26:81-104.

3. Elbaz A, Bower JH, Maraganore DM, McDonnell SK, Peterson BJ, Ahlskog JE, Schaid DJ, Rocca WA. Risk tables for parkinsonism and Parkinson's disease. J Clin Epidemiol. 2002;55(1):25-31.

4. Marsden CD. Neuromelanin and Parkinson's disease. J Neural Transm Suppl. 1983;19:121-141.

5. Fahn S. Description of Parkinson's disease as a clinical syndrome. Ann N Y Acad Sci. 2003;991:1-14.

6. Fearnley JM, Lees AJ. Ageing and Parkinson's disease: substantia nigra regional selectivity. Brain. 1991;114 ( Pt 5):2283-2301.

7. Chu Y, Kordower JH. Age-associated increases of alpha-synuclein in monkeys and humans are associated with nigrostriatal dopamine depletion: Is this the target for Parkinson's disease? Neurobiol Dis. 2007;25(1):134-149.

8. Hirsch EC. Biochemistry of Parkinson's disease with special reference to the dopaminergic systems. Mol Neurobiol. 1994;9(1-3):135-142.

9. Gai WP, Blumbergs PC, Geffen LB, Blessing WW. Age-related loss of dorsal vagal neurons in Parkinson's disease. Neurology. 1992;42(11):2106-2111.

10. Forno LS, Langston JW, DeLanney LE, Irwin I, Ricaurte GA. Locus ceruleus lesions and eosinophilic inclusions in MPTP-treated monkeys. Ann Neurol. 1986;20(4):449455.

11. Halliday GM, Blumbergs PC, Cotton RG, Blessing WW, Geffen LB. Loss of brainstem serotonin- and substance P-containing neurons in Parkinson's disease. Brain Res. 1990;510(1):104-107.

12. Wood-Kaczmar A, Gandhi S, Wood NW. Understanding the molecular causes of Parkinson's disease. Trends Mol. Med. 2006;12:521-528.

13. Sulzer D. Multiple hit hypotheses for dopamine neuron loss in Parkinson's disease. Trends Neurosci. 2007;30(5):244-250.

14. Spillantini MG, Crowther RA, Jakes R, Hasegawa M, Goedert M. alpha-Synuclein in filamentous inclusions of Lewy bodies from Parkinson's disease and dementia with lewy bodies. Proc Natl Acad Sci U S A. 1998;95(11):6469-6473.

15. Clinton LK, Blurton-Jones M, Myczek K, Trojanowski JQ, LaFerla FM. Synergistic Interactions between Abeta, tau, and alpha-synuclein: acceleration of neuropathology and cognitive decline. J Neurosci.30(21):7281-7289.

16. Markesbery WR, Jicha GA, Liu H, Schmitt FA. Lewy body pathology in normal elderly subjects. J Neuropathol Exp Neurol. 2009;68(7):816-822.

17. Abbott RD, Petrovitch H, White LR, Masaki KH, Tanner CM, Curb JD, Grandinetti A, Blanchette PL, Popper JS, Ross GW. Frequency of bowel movements and the future risk of Parkinson's disease. Neurology. 2001;57(3):456-462.

18. Ross GW, Abbott RD, Petrovitch H, Tanner CM, Davis DG, Nelson J, Markesbery WR, Hardman J, Masaki K, Launer L, White LR. Association of olfactory dysfunction with incidental Lewy bodies. Mov Disord. 2006;21(12):2062-2067.

19. Goldstein DS. Orthostatic hypotension as an early finding in Parkinson's disease. Clin Auton Res. 2006;16(1):46-54.

20. Tijero B, Gomez-Esteban JC, Llorens V, Lezcano E, Gonzalez-Fernandez MC, de Pancorbo MM, Ruiz-Martinez J, Cembellin JC, Zarranz JJ. Cardiac sympathetic denervation precedes nigrostriatal loss in the E46K mutation of the alpha-synuclein gene (SNCA). Clin Auton Res. 
21. Buob A, Winter H, Kindermann M, Becker G, Moller JC, Oertel WH, Bohm M. Parasympathetic but not sympathetic cardiac dysfunction at early stages of Parkinson's disease. Clin Res Cardiol.

22. Braak H. Staging of brain pathology related to sporadic Parkinson's disease. Neurobiol. Aging. 2003;24:197-211.

23. Kordower JH, Chu Y, Hauser RA, Freeman TB, Olanow CW. Lewy body-like pathology in long-term embryonic nigral transplants in Parkinson's disease. Nature Med. 2008;14:504-506.

24. Li JY, Englund E, Holton JL, Soulet D, Hagell P, Lees AJ, Lashley T, Quinn NP, Rehncrona S, Bjorklund A, Widner H, Revesz T, Lindvall O, Brundin P. Lewy bodies in grafted neurons in subjects with Parkinson's disease suggest host-to-graft disease propagation. Nat Med. 2008;14(5):501-503.

25. Desplats P, Lee H-J, Bae E-J, Patrick C, Rockenstein E, Crews L, Spencer B, Masliah $\mathrm{E}$, Lee S-J. Inclusion formation and neuronal cell death through neuron-to-neuron transmission of $\hat{\mathrm{I}}+$-synuclein. Proceedings of the National Academy of Sciences. 2009;106(31):13010-13015.

26. Liu J, Zhang J-P, Shi M, Quinn T, Bradner J, Beyer R, Chen S, Zhang J. Rab11a and HSP90 Regulate Recycling of Extracellular \{alpha\}-Synuclein. J. Neurosci. 2009;29(5):1480-1485.

27. Lee H-J, Suk J-E, Bae E-J, Lee J-H, Paik SR, Lee S-J. Assembly-dependent endocytosis and clearance of extracellular [alpha]-synuclein. The International Journal of Biochemistry \& Cell Biology. 2008;40(9):1835-1849.

28. Pan-Montojo F, Anichtchik O, Dening Y, Knels L, Pursche S, Jung R, Jackson S, Gille G, Spillantini MG, Reichmann H, Funk RHW. Progression of Parkinson's Disease Pathology Is Reproduced by Intragastric Administration of Rotenone in Mice. PLOS ONE.5(1):e8762.

29. Wood-Kaczmar A, Gandhi S, Wood NW. Understanding the molecular causes of Parkinson's disease. Trends in Molecular Medicine. 2006;12(11):521-528.

30. Krüger R, Sharma M, Riess O, Gasser T, Van Broeckhoven C, Theuns J, Aasly J, Annesi G, Bentivoglio AR, Brice A, Djarmati A, Elbaz A, Farrer M, Ferrarese C, Gibson JM, Hadjigeorgiou GM, Hattori N, Ioannidis JPA, Jasinska-Myga B, Klein C, Lambert J-C, Lesage S, Lin J-J, Lynch T, Mellick GD, de Nigris F, Opala G, Prigione A, Quattrone A, Ross OA, Satake W, Silburn PA, Tan EK, Toda T, Tomiyama H, Wirdefeldt K, Wszolek Z, Xiromerisiou G, Maraganore DM. A large-scale genetic association study to evaluate the contribution of Omi/HtrA2 (PARK13) to Parkinson's disease. Neurobiology of Aging.In Press, Corrected Proof.

31. Maroteaux L, Campanelli JT, Scheller RH. Synuclein: a neuron-specific protein localized to the nucleus and presynaptic nerve terminal. J Neurosci. 1988;8(8):28042815.

32. Ueda K, Fukushima H, Masliah E, Xia Y, Iwai A, Yoshimoto M, Otero DA, Kondo J, Ihara Y, Saitoh T. Molecular cloning of cDNA encoding an unrecognized component of amyloid in Alzheimer disease. Proc Natl Acad Sci U S A. 1993;90(23):1128211286.

33. George JM, Jin H, Woods WS, Clayton DF. Characterization of a novel protein regulated during the critical period for song learning in the zebra finch. Neuron. 1995;15(2):361-372.

34. Polymeropoulos MH, Lavedan C, Leroy E, Ide SE, Dehejia A, Dutra A, Pike B, Root H, Rubenstein J, Boyer R, Stenroos ES, Chandrasekharappa S, Athanassiadou A, Papapetropoulos T, Johnson WG, Lazzarini AM, Duvoisin RC, Di Iorio G, Golbe LI, 
Nussbaum RL. Mutation in the alpha-synuclein gene identified in families with Parkinson's disease. Science. 1997;276(5321):2045-2047.

35. Spillantini MG, Schmidt ML, Lee VM, Trojanowski JQ, Jakes R, Goedert M. Alphasynuclein in Lewy bodies. Nature. 1997;388(6645):839-840.

36. Kruger R, Kuhn W, Muller T, Woitalla D, Graeber M, Kosel S, Przuntek H, Epplen JT, Schols L, Riess O. Ala30Pro mutation in the gene encoding alpha-synuclein in Parkinson's disease. Nat Genet. 1998;18(2):106-108.

37. Zarranz JJ, Alegre J, Gomez-Esteban JC, Lezcano E, Ros R, Ampuero I, Vidal L, Hoenicka J, Rodriguez O, Atares B, Llorens V, Gomez Tortosa E, del Ser T, Munoz DG, de Yebenes JG. The new mutation, E46K, of alpha-synuclein causes Parkinson and Lewy body dementia. Ann Neurol. 2004;55(2):164-173.

38. Singleton AB, Farrer M, Johnson J, Singleton A, Hague S, Kachergus J, Hulihan M, Peuralinna T, Dutra A, Nussbaum R, Lincoln S, Crawley A, Hanson M, Maraganore D, Adler C, Cookson MR, Muenter M, Baptista M, Miller D, Blancato J, Hardy J, Gwinn-Hardy K. alpha-Synuclein locus triplication causes Parkinson's disease. Science. 2003;302(5646):841.

39. Chartier-Harlin MC, Kachergus J, Roumier C, Mouroux V, Douay X, Lincoln S, Levecque C, Larvor L, Andrieux J, Hulihan M, Waucquier N, Defebvre L, Amouyel P, Farrer M, Destee A. Alpha-synuclein locus duplication as a cause of familial Parkinson's disease. Lancet. 2004;364(9440):1167-1169.

40. Wszolek ZK, Pfeiffer RF, Tsuboi Y, Uitti RJ, McComb RD, Stoessl AJ, Strongosky AJ, Zimprich A, Muller-Myhsok B, Farrer MJ, Gasser T, Calne DB, Dickson DW. Autosomal dominant parkinsonism associated with variable synuclein and tau pathology. Neurology. 2004;62(9):1619-1622.

41. Farrer M, Kachergus J, Forno L, Lincoln S, Wang DS, Hulihan M, Maraganore D, Gwinn-Hardy K, Wszolek Z, Dickson D, Langston JW. Comparison of kindreds with parkinsonism and alpha-synuclein genomic multiplications. Ann Neurol. 2004;55(2):174-179.

42. Fuchs J. Phenotypic variation in a large Swedish pedigree due to SNCA duplication and triplication. Neurology. 2007;68:916-922.

43. Nuytemans K, Meeus B, Crosiers D, Brouwers N, Goossens D, Engelborghs S, Pals P, Pickut B, Van den Broeck M, Corsmit E, Cras P, De Deyn PP, Del-Favero J, Van Broeckhoven C, Theuns J. Relative contribution of simple mutations vs. copy number variations in five Parkinson disease genes in the Belgian population. Hum Mutat. 2009;30(7):1054-1061.

44. Mizuta I, Satake W, Nakabayashi Y, Ito C, Suzuki S, Momose Y, Nagai Y, Oka A, Inoko H, Fukae J, Saito Y, Sawabe M, Murayama S, Yamamoto M, Hattori N, Murata M, Toda T. Multiple candidate gene analysis identifies \{alpha\}-synuclein as a susceptibility gene for sporadic Parkinson's disease. Hum. Mol. Genet. 2006;15(7):1151-1158.

45. Lee HJ, Choi C, Lee SJ. Membrane-bound alpha-synuclein has a high aggregation propensity and the ability to seed the aggregation of the cytosolic form. $J$ Biol Chem. 2002;277(1):671-678.

46. Devi L, Raghavendran V, Prabhu BM, Avadhani NG, Anandatheerthavarada HK. Mitochondrial import and accumulation of alpha-synuclein impair complex I in human dopaminergic neuronal cultures and Parkinson disease brain. $J$ Biol Chem. 2008;283(14):9089-9100.

47. Giasson BI, Uryu K, Trojanowski JQ, Lee VM. Mutant and wild type human alphasynucleins assemble into elongated filaments with distinct morphologies in vitro. $J$ Biol Chem. 1999;274(12):7619-7622. 
48. Giasson BI, Murray IV, Trojanowski JQ, Lee VM. A hydrophobic stretch of 12 amino acid residues in the middle of alpha-synuclein is essential for filament assembly. $J$ Biol Chem. 2001;276(4):2380-2386.

49. Negro A, Brunati AM, Donella-Deana A, Massimino ML, Pinna LA. Multiple phosphorylation of alpha-synuclein by protein tyrosine kinase Syk prevents eosininduced aggregation. FASEB J. 2002;16(2):210-212.

50. Chen L, Periquet M, Wang X, Negro A, McLean PJ, Hyman BT, Feany MB. Tyrosine and serine phosphorylation of alpha-synuclein have opposing effects on neurotoxicity and soluble oligomer formation. J Clin Invest. 2009;119(11):3257-3265.

51. McFarland NR, Fan Z, Xu K, Schwarzschild MA, Feany MB, Hyman BT, McLean PJ. Alpha-synuclein S129 phosphorylation mutants do not alter nigrostriatal toxicity in a rat model of Parkinson disease. J Neuropathol Exp Neurol. 2009;68(5):515-524.

52. Azeredo da Silveira S, Schneider BL, Cifuentes-Diaz C, Sage D, Abbas-Terki T, Iwatsubo T, Unser M, Aebischer P. Phosphorylation does not prompt, nor prevent, the formation of alpha-synuclein toxic species in a rat model of Parkinson's disease. Hum Mol Genet. 2009;18(5):872-887.

53. Murphy DD, Rueter SM, Trojanowski JQ, Lee VM. Synucleins are developmentally expressed, and alpha-synuclein regulates the size of the presynaptic vesicular pool in primary hippocampal neurons. J Neurosci. 2000;20(9):3214-3220.

54. Abeliovich A, Schmitz Y, Farinas I, Choi-Lundberg D, Ho WH, Castillo PE, Shinsky N, Verdugo JM, Armanini M, Ryan A, Hynes M, Phillips H, Sulzer D, Rosenthal A. Mice lacking alpha-synuclein display functional deficits in the nigrostriatal dopamine system. Neuron. 2000;25(1):239-252.

55. Barcelo-Coblijn G, Golovko MY, Weinhofer I, Berger J, Murphy EJ. Brain neutral lipids mass is increased in alpha-synuclein gene-ablated mice. $J$ Neurochem. 2007;101(1):132-141.

56. Golovko MY, Barcelo-Coblijn G, Castagnet PI, Austin S, Combs CK, Murphy EJ. The role of alpha-synuclein in brain lipid metabolism: a downstream impact on brain inflammatory response. Mol Cell Biochem. 2009;326(1-2):55-66.

57. Golovko MY, Rosenberger TA, Faergeman NJ, Feddersen S, Cole NB, Pribill I, Berger J, Nussbaum RL, Murphy EJ. Acyl-CoA synthetase activity links wild-type but not mutant alpha-synuclein to brain arachidonate metabolism. Biochemistry. 2006;45(22):6956-6966.

58. Sharon R, Bar-Joseph I, Mirick GE, Serhan CN, Selkoe DJ. Altered fatty acid composition of dopaminergic neurons expressing alpha-synuclein and human brains with alpha-synucleinopathies. J Biol Chem. 2003;278(50):49874-49881.

59. Ben Gedalya T, Loeb V, Israeli E, Altschuler Y, Selkoe DJ, Sharon R. Alphasynuclein and polyunsaturated fatty acids promote clathrin-mediated endocytosis and synaptic vesicle recycling. Traffic. 2009;10(2):218-234.

60. Cooper AA, Gitler AD, Cashikar A, Haynes CM, Hill KJ, Bhullar B, Liu K, Xu K, Strathearn KE, Liu F, Cao S, Caldwell KA, Caldwell GA, Marsischky G, Kolodner RD, Labaer J, Rochet JC, Bonini NM, Lindquist S. Alpha-synuclein blocks ER-Golgi traffic and Rab1 rescues neuron loss in Parkinson's models. Science. 2006;313(5785):324-328.

61. Gitler AD, Bevis BJ, Shorter J, Strathearn KE, Hamamichi S, Su LJ, Caldwell KA, Caldwell GA, Rochet JC, McCaffery JM, Barlowe C, Lindquist S. The Parkinson's disease protein alpha-synuclein disrupts cellular Rab homeostasis. Proc Natl Acad Sci U S A. 2008;105(1):145-150. 
62. Chandra S, Gallardo G, Fernández-Chacón R, Schlüter OM, Südhof TC. [alpha]Synuclein Cooperates with CSP[alpha] in Preventing Neurodegeneration. Cell. 2005;123(3):383-396.

63. Quilty MC, King AE, Gai WP, Pountney DL, West AK, Vickers JC, Dickson TC. Alpha-synuclein is upregulated in neurones in response to chronic oxidative stress and is associated with neuroprotection. Exp Neurol. 2006;199(2):249-256.

64. Vartiainen S, Aarnio V, Lakso M, Wong G. Increased lifespan in transgenic Caenorhabditis elegans overexpressing human alpha-synuclein. Exp Gerontol. 2006;41(9):871-876.

65. Seo JH, Rah JC, Choi SH, Shin JK, Min K, Kim HS, Park CH, Kim S, Kim EM, Lee SH, Lee S, Suh SW, Suh YH. Alpha-synuclein regulates neuronal survival via Bcl-2 family expression and PI3/Akt kinase pathway. FASEB J. 2002;16(13):1826-1828.

66. Bayir H, Kapralov AA, Jiang J, Huang Z, Tyurina YY, Tyurin VA, Zhao Q, Belikova NA, Vlasova, II, Maeda A, Zhu J, Na HM, Mastroberardino PG, Sparvero LJ, Amoscato AA, Chu CT, Greenamyre JT, Kagan VE. Peroxidase mechanism of lipiddependent cross-linking of synuclein with cytochrome $\mathrm{C}$ : protection against apoptosis versus delayed oxidative stress in Parkinson disease. $J$ Biol Chem. 2009;284(23):15951-15969.

67. Ostrerova N, Petrucelli L, Farrer M, Mehta N, Choi P, Hardy J, Wolozin B. alphaSynuclein shares physical and functional homology with 14-3-3 proteins. J Neurosci. 1999;19(14):5782-5791.

68. Perez RG, Waymire JC, Lin E, Liu JJ, Guo F, Zigmond MJ. A role for alpha-synuclein in the regulation of dopamine biosynthesis. J Neurosci. 2002;22(8):3090-3099.

69. Lee FJ, Liu F, Pristupa ZB, Niznik HB. Direct binding and functional coupling of alpha-synuclein to the dopamine transporters accelerate dopamine-induced apoptosis. FASEB J. 2001;15(6):916-926.

70. Loeb V, Yakunin E, Saada A, Sharon R. The Transgenic Overexpression of $\hat{\mathrm{I}} \pm-$ Synuclein and Not Its Related Pathology Associates with Complex I Inhibition. Journal of Biological Chemistry.285(10):7334-7343.

71. Ellis CE, Murphy EJ, Mitchell DC, Golovko MY, Scaglia F, Barcelo-Coblijn GC, Nussbaum RL. Mitochondrial lipid abnormality and electron transport chain impairment in mice lacking alpha-synuclein. Mol Cell Biol. 2005;25(22):1019010201.

72. Klivenyi P, Siwek D, Gardian G, Yang L, Starkov A, Cleren C, Ferrante RJ, Kowall NW, Abeliovich A, Beal MF. Mice lacking alpha-synuclein are resistant to mitochondrial toxins. Neurobiol Dis. 2006;21(3):541-548.

73. Lucking CB, Brice A. Alpha-synuclein and Parkinson's disease. Cell Mol Life Sci. 2000;57(13-14):1894-1908.

74. Dawson TM, Dawson VL. The role of parkin in familial and sporadic Parkinson's disease. Mov Disord.25 Suppl 1:S32-39.

75. Abou-Sleiman PM, Muqit MM, Wood NW. Expanding insights of mitochondrial dysfunction in Parkinson's disease. Nat Rev Neurosci. 2006;7(3):207-219.

76. Oliveira SA, Scott WK, Nance MA, Watts RL, Hubble JP, Koller WC, Lyons KE, Pahwa R, Stern MB, Hiner BC, Jankovic J, Ondo WG, Allen FH, Jr., Scott BL, Goetz CG, Small GW, Mastaglia FL, Stajich JM, Zhang F, Booze MW, Reaves JA, Middleton LT, Haines JL, Pericak-Vance MA, Vance JM, Martin ER. Association study of Parkin gene polymorphisms with idiopathic Parkinson disease. Arch Neurol. 2003;60(7):975-980. 
77. Mori H, Kondo T, Yokochi M, Matsumine H, Nakagawa-Hattori Y, Miyake T, Suda K, Mizuno Y. Pathologic and biochemical studies of juvenile parkinsonism linked to chromosome 6q. Neurology. 1998;51(3):890-892.

78. Hattori N, Kitada T, Matsumine H, Asakawa S, Yamamura Y, Yoshino H, Kobayashi T, Yokochi M, Wang M, Yoritaka A, Kondo T, Kuzuhara S, Nakamura S, Shimizu N, Mizuno Y. Molecular genetic analysis of a novel Parkin gene in Japanese families with autosomal recessive juvenile parkinsonism: evidence for variable homozygous deletions in the Parkin gene in affected individuals. Ann Neurol. 1998;44(6):935-941.

79. Kitada T, Asakawa S, Hattori N, Matsumine H, Yamamura Y, Minoshima S, Yokochi M, Mizuno Y, Shimizu N. Mutations in the parkin gene cause autosomal recessive juvenile parkinsonism. Nature. 1998;392(6676):605-608.

80. Shimura H, Hattori N, Kubo S, Mizuno Y, Asakawa S, Minoshima S, Shimizu N, Iwai K, Chiba T, Tanaka K, Suzuki T. Familial Parkinson disease gene product, parkin, is a ubiquitin-protein ligase. Nat Genet. 2000;25(3):302-305.

81. Imai Y, Soda M, Inoue H, Hattori N, Mizuno Y, Takahashi R. An unfolded putative transmembrane polypeptide, which can lead to endoplasmic reticulum stress, is a substrate of Parkin. Cell. 2001;105(7):891-902.

82. Choi P, Snyder H, Petrucelli L, Theisler C, Chong M, Zhang Y, Lim K, Chung KK, Kehoe K, D'Adamio L, Lee JM, Cochran E, Bowser R, Dawson TM, Wolozin B. SEPT5_v2 is a parkin-binding protein. Brain Res Mol Brain Res. 2003;117(2):179189.

83. Chung KK, Zhang Y, Lim KL, Tanaka Y, Huang H, Gao J, Ross CA, Dawson VL, Dawson TM. Parkin ubiquitinates the alpha-synuclein-interacting protein, synphilin-1: implications for Lewy-body formation in Parkinson disease. Nat Med. 2001;7(10):1144-1150.

84. Olzmann JA, Li L, Chudaev MV, Chen J, Perez FA, Palmiter RD, Chin LS. Parkinmediated K63-linked polyubiquitination targets misfolded DJ-1 to aggresomes via binding to HDAC6. J Cell Biol. 2007;178(6):1025-1038.

85. Pandey UB, Batlevi Y, Baehrecke EH, Taylor JP. HDAC6 at the intersection of autophagy, the ubiquitin-proteasome system and neurodegeneration. Autophagy. 2007;3(6):643-645.

86. Pandey UB, Nie Z, Batlevi Y, McCray BA, Ritson GP, Nedelsky NB, Schwartz SL, DiProspero NA, Knight MA, Schuldiner O, Padmanabhan R, Hild M, Berry DL, Garza D, Hubbert CC, Yao TP, Baehrecke EH, Taylor JP. HDAC6 rescues neurodegeneration and provides an essential link between autophagy and the UPS. Nature. 2007;447(7146):859-863.

87. Greene JC, Whitworth AJ, Kuo I, Andrews LA, Feany MB, Pallanck LJ. Mitochondrial pathology and apoptotic muscle degeneration in Drosophila parkin mutants. Proc Natl Acad Sci U S A. 2003;100(7):4078-4083.

88. Narendra D, Tanaka A, Suen DF, Youle RJ. Parkin is recruited selectively to impaired mitochondria and promotes their autophagy. J Cell Biol. 2008;183(5):795-803.

89. Lee J-Y, Nagano Y, Taylor JP, Lim KL, Yao T-P. Disease-causing mutations in Parkin impair mitochondrial ubiquitination, aggregation, and HDAC6-dependent mitophagy. The Journal of Cell Biology.189(4):671-679.

90. Mizuno Y, Hattori N, Kubo S, Sato S, Nishioka K, Hatano T, Tomiyama H, Funayama M, Machida Y, Mochizuki H. Progress in the pathogenesis and genetics of Parkinson's disease. Philos Trans R Soc Lond B Biol Sci. 2008;363(1500):2215-2227.

91. Valente EM, Salvi S, Ialongo T, Marongiu R, Elia AE, Caputo V, Romito L, Albanese A, Dallapiccola B, Bentivoglio AR. PINK1 mutations are associated with sporadic early-onset parkinsonism. Ann Neurol. 2004;56(3):336-341. 
92. Nuytemans K, Theuns J, Cruts M, Van Broeckhoven C. Genetic etiology of Parkinson disease associated with mutations in the SNCA, PARK2, PINK1, PARK7, and LRRK2 genes: a mutation update. Hum Mutat.

93. Li J, Yen C, Liaw D, Podsypanina K, Bose S, Wang SI, Puc J, Miliaresis C, Rodgers L, McCombie R, Bigner SH, Giovanella BC, Ittmann M, Tycko B, Hibshoosh H, Wigler MH, Parsons R. PTEN, a putative protein tyrosine phosphatase gene mutated in human brain, breast, and prostate cancer. Science. 1997;275(5308):1943-1947.

94. Dodson MW, Guo M. Pink1, Parkin, DJ-1 and mitochondrial dysfunction in Parkinson's disease. Current Opinion in Neurobiology. 2007;17(3):331-337.

95. Deng H, Jankovic J, Guo Y, Xie W, Le W. Small interfering RNA targeting the PINK1 induces apoptosis in dopaminergic cells SH-SY5Y. Biochem Biophys Res Commun. 2005;337(4):1133-1138.

96. Hoepken H-H, Gispert S, Azizov M, Klinkenberg M, Ricciardi F, Kurz A, MoralesGordo B, Bonin M, Riess O, Gasser T, Kögel D, Steinmetz H, Auburger G. Parkinson patient fibroblasts show increased alpha-synuclein expression. Experimental Neurology. 2008;212(2):307-313.

97. Muqit MM, Abou-Sleiman PM, Saurin AT, Harvey K, Gandhi S, Deas E, Eaton S, Payne Smith MD, Venner K, Matilla A, Healy DG, Gilks WP, Lees AJ, Holton J, Revesz T, Parker PJ, Harvey RJ, Wood NW, Latchman DS. Altered cleavage and localization of PINK1 to aggresomes in the presence of proteasomal stress. $J$ Neurochem. 2006;98(1):156-169.

98. Clark IE, Dodson MW, Jiang C, Cao JH, Huh JR, Seol JH, Yoo SJ, Hay BA, Guo M. Drosophila pink1 is required for mitochondrial function and interacts genetically with parkin. Nature. 2006;441(7097):1162-1166.

99. Park J, Lee SB, Lee S, Kim Y, Song S, Kim S, Bae E, Kim J, Shong M, Kim JM, Chung J. Mitochondrial dysfunction in Drosophila PINK1 mutants is complemented by parkin. Nature. 2006;441(7097):1157-1161.

100. Yang Y, Gehrke S, Imai Y, Huang Z, Ouyang Y, Wang JW, Yang L, Beal MF, Vogel $\mathrm{H}, \mathrm{Lu}$ B. Mitochondrial pathology and muscle and dopaminergic neuron degeneration caused by inactivation of Drosophila Pink1 is rescued by Parkin. Proc Natl Acad Sci U $S$ A. 2006;103(28):10793-10798.

101. Gautier CA, Kitada T, Shen J. Loss of PINK1 causes mitochondrial functional defects and increased sensitivity to oxidative stress. Proc Natl Acad Sci $U$ S A. 2008;105(32):11364-11369.

102. Wood-Kaczmar A, Gandhi S, Yao Z, Abramov AY, Miljan EA, Keen G, Stanyer L, Hargreaves I, Klupsch K, Deas E, Downward J, Mansfield L, Jat P, Taylor J, Heales S, Duchen MR, Latchman D, Tabrizi SJ, Wood NW. PINK1 is necessary for long term survival and mitochondrial function in human dopaminergic neurons. PLoS One. 2008;3(6):e2455.

103. Pridgeon JW, Olzmann JA, Chin L-S, Li L. PINK1 Protects against Oxidative Stress by Phosphorylating Mitochondrial Chaperone TRAP1. PLoS Biol. 2007;5(7):e172.

104. Leav I, Plescia J, Goel HL, Li J, Jiang Z, Cohen RJ, Languino LR, Altieri DC. Cytoprotective mitochondrial chaperone TRAP-1 as a novel molecular target in localized and metastatic prostate cancer. Am J Pathol.176(1):393-401.

105. Montesano Gesualdi N, Chirico G, Pirozzi G, Costantino E, Landriscina M, Esposito F. Tumor necrosis factor-associated protein 1 (TRAP-1) protects cells from oxidative stress and apoptosis. Stress. 2007;10(4):342-350.

106. Im CN, Lee JS, Zheng Y, Seo JS. Iron chelation study in a normal human hepatocyte cell line suggests that tumor necrosis factor receptor-associated protein 1 (TRAP1) 
regulates production of reactive oxygen species. J Cell Biochem. 2007;100(2):474486.

107. van Ham TJ, Thijssen KL, Breitling R, Hofstra RMW, Plasterk RHA, Nollen EAA. C. elegans Model Identifies Genetic Modifiers of Ît-Synuclein Inclusion Formation During Aging. PLoS Genetics. 2008;4(3):e1000027.

108. Plun-Favreau H, Klupsch K, Moisoi N, Gandhi S, Kjaer S, Frith D, Harvey K, Deas E, Harvey RJ, McDonald N, Wood NW, Martins LM, Downward J. The mitochondrial protease HtrA2 is regulated by Parkinson's disease-associated kinase PINK1. Nat Cell Biol. 2007;9(11):1243-1252.

109. Matsuda N, Sato S, Shiba K, Okatsu K, Saisho K, Gautier CA, Sou YS, Saiki S, Kawajiri S, Sato F, Kimura M, Komatsu M, Hattori N, Tanaka K. PINK1 stabilized by mitochondrial depolarization recruits Parkin to damaged mitochondria and activates latent Parkin for mitophagy. J Cell Biol.189(2):211-221.

110. Narendra DP, Jin SM, Tanaka A, Suen DF, Gautier CA, Shen J, Cookson MR, Youle RJ. PINK1 is selectively stabilized on impaired mitochondria to activate Parkin. PLoS Biol.8(1):e1000298.

111. Bonifati V, Rizzu P, Squitieri F, Krieger E, Vanacore N, van Swieten JC, Brice A, van Duijn CM, Oostra B, Meco G, Heutink P. DJ-1( PARK7), a novel gene for autosomal recessive, early onset parkinsonism. Neurol Sci. 2003;24(3):159-160.

112. Xu J, Zhong N, Wang H, Elias JE, Kim CY, Woldman I, Pifl C, Gygi SP, Geula C, Yankner BA. The Parkinson's disease-associated DJ-1 protein is a transcriptional coactivator that protects against neuronal apoptosis. Hum Mol Genet. 2005;14(9):12311241.

113. Büeler H. Impaired mitochondrial dynamics and function in the pathogenesis of Parkinson's disease. Experimental Neurology. 2009;218(2):235-246.

114. Menzies FM, Yenisetti SC, Min K-T. Roles of Drosophila DJ-1 in Survival of Dopaminergic Neurons and Oxidative Stress. 2005;15(17):1578-1582.

115. Kim RH, Smith PD, Aleyasin H, Hayley S, Mount MP, Pownall S, Wakeham A, YouTen AJ, Kalia SK, Horne P, Westaway D, Lozano AM, Anisman H, Park DS, Mak TW. Hypersensitivity of DJ-1-deficient mice to 1-methyl-4-phenyl-1,2,3,6tetrahydropyrindine (MPTP) and oxidative stress. Proc Natl Acad Sci U S A. 2005;102(14):5215-5220.

116. Moore DJ, Zhang L, Troncoso J, Lee MK, Hattori N, Mizuno Y, Dawson TM, Dawson VL. Association of DJ-1 and parkin mediated by pathogenic DJ-1 mutations and oxidative stress. Hum Mol Genet. 2005;14(1):71-84.

117. Xiong H, Wang D, Chen L, Choo YS, Ma H, Tang C, Xia K, Jiang W, Ronai Z, Zhuang X, Zhang Z. Parkin, PINK1, and DJ-1 form a ubiquitin E3 ligase complex promoting unfolded protein degradation. J Clin Invest. 2009;119(3):650-660.

118. Tang B, Xiong H, Sun P, Zhang Y, Wang D, Hu Z, Zhu Z, Ma H, Pan Q, Xia JH, Xia $\mathrm{K}$, Zhang Z. Association of PINK1 and DJ-1 confers digenic inheritance of early-onset Parkinson's disease. Hum Mol Genet. 2006;15(11):1816-1825.

119. Bandopadhyay R, de Belleroche J. Pathogenesis of Parkinson's disease: emerging role of molecular chaperones. Trends in Molecular Medicine. 2009;16(1):27-36.

120. Cookson MR. alpha-Synuclein and neuronal cell death. Mol Neurodegener. 2009;4:9.

121. Conway KA, Harper JD, Lansbury PT. Accelerated in vitro fibril formation by a mutant alpha-synuclein linked to early-onset Parkinson disease. Nat Med. 1998;4(11):1318-1320.

122. Conway KA, Lee SJ, Rochet JC, Ding TT, Williamson RE, Lansbury PT, Jr. Acceleration of oligomerization, not fibrillization, is a shared property of both alpha- 
synuclein mutations linked to early-onset Parkinson's disease: implications for pathogenesis and therapy. Proc Natl Acad Sci U S A. 2000;97(2):571-576.

123. Choi W, Zibaee S, Jakes R, Serpell LC, Davletov B, Crowther RA, Goedert M. Mutation E46K increases phospholipid binding and assembly into filaments of human alpha-synuclein. FEBS Lett. 2004;576(3):363-368.

124. Fredenburg RA, Rospigliosi C, Meray RK, Kessler JC, Lashuel HA, Eliezer D, Lansbury PT, Jr. The impact of the E46K mutation on the properties of alphasynuclein in its monomeric and oligomeric states. Biochemistry. 2007;46(24):71077118.

125. Chau KY, Ching HL, Schapira AH, Cooper JM. Relationship between alpha synuclein phosphorylation, proteasomal inhibition and cell death: relevance to Parkinson's disease pathogenesis. J Neurochem. 2009;110(3):1005-1013.

126. Chen L, Feany MB. Alpha-synuclein phosphorylation controls neurotoxicity and inclusion formation in a Drosophila model of Parkinson disease. Nat Neurosci. 2005;8(5):657-663.

127. Kahle PJ, Neumann M, Ozmen L, Muller V, Jacobsen H, Schindzielorz A, Okochi M, Leimer U, van Der Putten H, Probst A, Kremmer E, Kretzschmar HA, Haass C. Subcellular localization of wild-type and Parkinson's disease-associated mutant alpha synuclein in human and transgenic mouse brain. J Neurosci. 2000;20(17):6365-6373.

128. Lakso M, Vartiainen S, Moilanen AM, Sirvio J, Thomas JH, Nass R, Blakely RD, Wong G. Dopaminergic neuronal loss and motor deficits in Caenorhabditis elegans overexpressing human alpha-synuclein. J Neurochem. 2003;86(1):165-172.

129. Hsu LJ, Sagara Y, Arroyo A, Rockenstein E, Sisk A, Mallory M, Wong J, Takenouchi T, Hashimoto M, Masliah E. \{alpha\}-Synuclein Promotes Mitochondrial Deficit and Oxidative Stress. Am J Pathol. 2000;157(2):401-410.

130. Smith WW, Jiang H, Pei Z, Tanaka Y, Morita H, Sawa A, Dawson VL, Dawson TM, Ross CA. Endoplasmic reticulum stress and mitochondrial cell death pathways mediate A53T mutant alpha-synuclein-induced toxicity. Hum. Mol. Genet. 2005;14(24):3801-3811.

131. Song DD, Shults CW, Sisk A, Rockenstein E, Masliah E. Enhanced substantia nigra mitochondrial pathology in human alpha-synuclein transgenic mice after treatment with MPTP. Exp Neurol. 2004;186(2):158-172.

132. Norris EH, Uryu K, Leight S, Giasson BI, Trojanowski JQ, Lee VM. Pesticide exposure exacerbates alpha-synucleinopathy in an A53T transgenic mouse model. Am J Pathol. 2007;170(2):658-666.

133. Yu WH, Matsuoka Y, Sziraki I, Hashim A, Lafrancois J, Sershen H, Duff KE. Increased dopaminergic neuron sensitivity to 1-methyl-4-phenyl-1,2,3,6tetrahydropyridine (MPTP) in transgenic mice expressing mutant A53T alphasynuclein. Neurochem Res. 2008;33(5):902-911.

134. Martin LJ, Pan Y, Price AC, Sterling W, Copeland NG, Jenkins NA, Price DL, Lee MK. Parkinson's Disease \{alpha\}-Synuclein Transgenic Mice Develop Neuronal Mitochondrial Degeneration and Cell Death. J. Neurosci. 2006;26(1):41-50.

135. Feany MB, Bender WW. A Drosophila model of Parkinson's disease. Nature. 2000;404(6776):394-398.

136. Lo Bianco C, Ridet JL, Schneider BL, Deglon N, Aebischer P. alpha -Synucleinopathy and selective dopaminergic neuron loss in a rat lentiviral-based model of Parkinson's disease. Proc Natl Acad Sci U S A. 2002;99(16):10813-10818.

137. Eslamboli A, Romero-Ramos M, Burger C, Bjorklund T, Muzyczka N, Mandel RJ, Baker H, Ridley RM, Kirik D. Long-term consequences of human alpha-synuclein overexpression in the primate ventral midbrain. Brain. 2007;130(Pt 3):799-815. 
138. Volles MJ, Lansbury JPT. Relationships between the Sequence of [alpha]-Synuclein and its Membrane Affinity, Fibrillization Propensity, and Yeast Toxicity. Journal of Molecular Biology. 2007;366(5):1510-1522.

139. Outeiro TF, Putcha P, Tetzlaff JE, Spoelgen R, Koker M, Carvalho F, Hyman BT, McLean PJ. Formation of toxic oligomeric alpha-synuclein species in living cells. PLoS One. 2008;3(4):e1867.

140. Danzer KM, Haasen D, Karow AR, Moussaud S, Habeck M, Giese A, Kretzschmar H, Hengerer B, Kostka M. Different species of alpha-synuclein oligomers induce calcium influx and seeding. J Neurosci. 2007;27(34):9220-9232.

141. Karpinar DP, Balija MB, Kugler S, Opazo F, Rezaei-Ghaleh N, Wender N, Kim HY, Taschenberger G, Falkenburger BH, Heise H, Kumar A, Riedel D, Fichtner L, Voigt A, Braus GH, Giller K, Becker S, Herzig A, Baldus M, Jackle H, Eimer S, Schulz JB, Griesinger C, Zweckstetter M. Pre-fibrillar alpha-synuclein variants with impaired beta-structure increase neurotoxicity in Parkinson's disease models. EMBO J. 2009;28(20):3256-3268.

142. Auluck PK, Chan HY, Trojanowski JQ, Lee VM, Bonini NM. Chaperone suppression of alpha-synuclein toxicity in a Drosophila model for Parkinson's disease. Science. 2002;295(5556):865-868.

143. Furukawa K, Matsuzaki-Kobayashi M, Hasegawa T, Kikuchi A, Sugeno N, Itoyama Y, Wang Y, Yao PJ, Bushlin I, Takeda A. Plasma membrane ion permeability induced by mutant alpha-synuclein contributes to the degeneration of neural cells. $J$ Neurochem. 2006;97(4):1071-1077.

144. Cuervo AM, Stefanis L, Fredenburg R, Lansbury PT, Sulzer D. Impaired degradation of mutant alpha-synuclein by chaperone-mediated autophagy. Science. 2004;305(5688):1292-1295.

145. Martinez-Vicente M, Talloczy Z, Kaushik S, Massey AC, Mazzulli J, Mosharov EV, Hodara R, Fredenburg R, Wu DC, Follenzi A, Dauer W, Przedborski S, Ischiropoulos H, Lansbury PT, Sulzer D, Cuervo AM. Dopamine-modified alpha-synuclein blocks chaperone-mediated autophagy. J Clin Invest. 2008;118(2):777-788.

146. Yang Q, She H, Gearing M, Colla E, Lee M, Shacka JJ, Mao Z. Regulation of neuronal survival factor MEF2D by chaperone-mediated autophagy. Science. 2009;323(5910):124-127.

147. McNaught KS, Perl DP, Brownell AL, Olanow CW. Systemic exposure to proteasome inhibitors causes a progressive model of Parkinson's disease. Ann Neurol. 2004;56(1):149-162.

148. Rogers N, Paine S, Bedford L, Layfield R. Review: the ubiquitin-proteasome system: contributions to cell death or survival in neurodegeneration. Neuropathol Appl Neurobiol.36(2):113-124.

149. Petrucelli L, O'Farrell C, Lockhart PJ, Baptista M, Kehoe K, Vink L, Choi P, Wolozin B, Farrer M, Hardy J, Cookson MR. Parkin protects against the toxicity associated with mutant alpha-synuclein: proteasome dysfunction selectively affects catecholaminergic neurons. Neuron. 2002;36(6):1007-1019.

150. Stefanis L, Larsen KE, Rideout HJ, Sulzer D, Greene LA. Expression of A53T mutant but not wild-type alpha-synuclein in PC12 cells induces alterations of the ubiquitindependent degradation system, loss of dopamine release, and autophagic cell death. $J$ Neurosci. 2001;21(24):9549-9560.

151. Snyder H, Mensah K, Theisler C, Lee J, Matouschek A, Wolozin B. Aggregated and monomeric alpha-synuclein bind to the S6' proteasomal protein and inhibit proteasomal function. J Biol Chem. 2003;278(14):11753-11759. 
152. Tanaka Y, Engelender S, Igarashi S, Rao RK, Wanner T, Tanzi RE, Sawa A, V LD, Dawson TM, Ross CA. Inducible expression of mutant alpha-synuclein decreases proteasome activity and increases sensitivity to mitochondria-dependent apoptosis. Hum Mol Genet. 2001;10(9):919-926.

153. Rott R, Szargel R, Haskin J, Shani V, Shainskaya A, Manov I, Liani E, Avraham E, Engelender S. Monoubiquitylation of alpha-synuclein by seven in absentia homolog (SIAH) promotes its aggregation in dopaminergic cells. $J$ Biol Chem. 2008;283(6):3316-3328.

154. Sulzer D. Clues to how alpha-synuclein damages neurons in Parkinson's disease. Mov Disord.25 Suppl 1:S27-31.

155. Inden M, Kitamura $Y$, Takeuchi $H$, Yanagida $T$, Takata K, Kobayashi $Y$, Taniguchi $T$, Yoshimoto K, Kaneko M, Okuma Y, Taira T, Ariga H, Shimohama S. Neurodegeneration of mouse nigrostriatal dopaminergic system induced by repeated oral administration of rotenone is prevented by 4-phenylbutyrate, a chemical chaperone. J Neurochem. 2007;101(6):1491-1504.

156. Abdulwahid Arif I, Ahmad Khan H. Environmental toxins and Parkinson's disease: Putative roles of impaired electron transport chain and oxidative stress. Toxicology and Industrial Health.26(2):121-128.

157. Semchuk KM, Love EJ, Lee RG. Parkinson's disease and exposure to agricultural work and pesticide chemicals. Neurology. 1992;42(7):1328-1335.

158. Liou HH, Tsai MC, Chen CJ, Jeng JS, Chang YC, Chen SY, Chen RC. Environmental risk factors and Parkinson's disease: a case-control study in Taiwan. Neurology. 1997;48(6):1583-1588.

159. Costello S, Cockburn M, Bronstein J, Zhang X, Ritz B. Parkinson's disease and residential exposure to maneb and paraquat from agricultural applications in the central valley of California. Am J Epidemiol. 2009;169(8):919-926.

160. Gash DM, Rutland K, Hudson NL, Sullivan PG, Bing G, Cass WA, Pandya JD, Liu M, Choi DY, Hunter RL, Gerhardt GA, Smith CD, Slevin JT, Prince TS. Trichloroethylene: Parkinsonism and complex 1 mitochondrial neurotoxicity. Ann Neurol. 2008;63(2):184-192.

161. Schapira AH, Cooper JM, Dexter D, Clark JB, Jenner P, Marsden CD. Mitochondrial complex I deficiency in Parkinson's disease. J Neurochem. 1990;54(3):823-827.

162. Mizuno Y, Ikebe S, Hattori N, Nakagawa-Hattori Y, Mochizuki H, Tanaka M, Ozawa T. Role of mitochondria in the etiology and pathogenesis of Parkinson's disease. Biochim Biophys Acta. 1995;1271(1):265-274.

163. Smith CD, Carney JM, Starke-Reed PE, Oliver CN, Stadtman ER, Floyd RA, Markesbery WR. Excess brain protein oxidation and enzyme dysfunction in normal aging and in Alzheimer disease. Proc Natl Acad Sci U S A. 1991;88(23):10540-10543.

164. Oliver CN, Ahn BW, Moerman EJ, Goldstein S, Stadtman ER. Age-related changes in oxidized proteins. J Biol Chem. 1987;262(12):5488-5491.

165. Malkus KA, Tsika E, Ischiropoulos $\mathrm{H}$. Oxidative modifications, mitochondrial dysfunction, and impaired protein degradation in Parkinson's disease: how neurons are lost in the Bermuda triangle. Mol Neurodegener. 2009;4:24.

166. Keeney PM, Xie J, Capaldi RA, Bennett JP, Jr. Parkinson's disease brain mitochondrial complex I has oxidatively damaged subunits and is functionally impaired and misassembled. J Neurosci. 2006;26(19):5256-5264.

167. Lotharius J, Brundin P. Impaired dopamine storage resulting from alpha-synuclein mutations may contribute to the pathogenesis of Parkinson's disease. Hum Mol Genet. 2002;11(20):2395-2407. 
168. Conway KA, Rochet JC, Bieganski RM, Lansbury PT, Jr. Kinetic stabilization of the alpha-synuclein protofibril by a dopamine-alpha-synuclein adduct. Science. 2001;294(5545):1346-1349.

169. Moussa CEH, Mahmoodian F, Tomita Y, Sidhu A. Dopamine differentially induces aggregation of A53T mutant and wild type [alpha]-synuclein: Insights into the protein chemistry of Parkinson's disease. Biochemical and Biophysical Research Communications. 2008;365(4):833-839.

170. Park SM, Jung HY, Kim TD, Park JH, Yang CH, Kim J. Distinct roles of the Nterminal-binding domain and the C-terminal-solubilizing domain of alpha-synuclein, a molecular chaperone. J Biol Chem. 2002;277(32):28512-28520.

171. Wang D, Qian L, Xiong H, Liu J, Neckameyer WS, Oldham S, Xia K, Wang J, Bodmer R, Zhang Z. Antioxidants protect PINK1-dependent dopaminergic neurons in Drosophila. Proceedings of the National Academy of Sciences. 2006;103(36):1352013525.

172. Whitworth AJ, Theodore DA, Greene JC, Benes H, Wes PD, Pallanck LJ. Increased glutathione S-transferase activity rescues dopaminergic neuron loss in a Drosophila model of Parkinson's disease. Proc Natl Acad Sci U S A. 2005;102(22):8024-8029.

173. Li WW, Yang R, Guo JC, Ren HM, Zha XL, Cheng JS, Cai DF. Localization of alphasynuclein to mitochondria within midbrain of mice. Neuroreport. 2007;18(15):15431546.

174. Liu G, Zhang C, Yin J, Li X, Cheng F, Li Y, Yang H, Ueda K, Chan P, Yu S. alphaSynuclein is differentially expressed in mitochondria from different rat brain regions and dose-dependently down-regulates complex I activity. Neurosci Lett. 2009;454(3):187-192.

175. Parihar MS, Parihar A, Fujita M, Hashimoto M, Ghafourifar P. Mitochondrial association of alpha-synuclein causes oxidative stress. Cell Mol Life Sci. 2008;65(78):1272-1284.

176. Parihar MS, Parihar A, Fujita M, Hashimoto M, Ghafourifar P. Alpha-synuclein overexpression and aggregation exacerbates impairment of mitochondrial functions by augmenting oxidative stress in human neuroblastoma cells. The International Journal of Biochemistry \& Cell Biology. 2009;41(10):2015-2024.

177. Shavali S, Brown-Borg HM, Ebadi M, Porter J. Mitochondrial localization of alphasynuclein protein in alpha-synuclein overexpressing cells. Neurosci Lett. 2008;439(2):125-128.

178. Dauer W, Kholodilov N, Vila M, Trillat AC, Goodchild R, Larsen KE, Staal R, Tieu K, Schmitz Y, Yuan CA, Rocha M, Jackson-Lewis V, Hersch S, Sulzer D, Przedborski S, Burke R, Hen R. Resistance of alpha -synuclein null mice to the parkinsonian neurotoxin MPTP. Proc Natl Acad Sci U S A. 2002;99(22):14524-14529.

179. Stichel CC, Zhu X-R, Bader V, Linnartz B, Schmidt S, Lubbert H. Mono- and doublemutant mouse models of Parkinson's disease display severe mitochondrial damage. Hum. Mol. Genet. 2007;16(20):2377-2393.

180. Cole NB, Dieuliis D, Leo P, Mitchell DC, Nussbaum RL. Mitochondrial translocation of alpha-synuclein is promoted by intracellular acidification. Exp Cell Res. 2008;314(10):2076-2089.

181. Falkenburger BH SJ. Limitations of cellular models in Parkinson's disease research. Journal of Neural Transmission. 2006;70:261-268.

182. Hirth F. Drosophila melanogaster in the Study of Human Neurodegeneration. CNS Neurol Disord Drug Targets.

183. Rubin GM, Yandell MD, Wortman JR, Gabor Miklos GL, Nelson CR, Hariharan IK, Fortini ME, Li PW, Apweiler R, Fleischmann W, Cherry JM, Henikoff S, Skupski 
MP, Misra S, Ashburner M, Birney E, Boguski MS, Brody T, Brokstein P, Celniker SE, Chervitz SA, Coates D, Cravchik A, Gabrielian A, Galle RF, Gelbart WM, George RA, Goldstein LS, Gong F, Guan P, Harris NL, Hay BA, Hoskins RA, Li J, Li Z, Hynes RO, Jones SJ, Kuehl PM, Lemaitre B, Littleton JT, Morrison DK, Mungall C, O'Farrell PH, Pickeral OK, Shue C, Vosshall LB, Zhang J, Zhao Q, Zheng XH, Lewis S. Comparative genomics of the eukaryotes. Science. 2000;287(5461):22042215.

184. Muqit MM, Feany MB. Modelling neurodegenerative diseases in Drosophila: a fruitful approach? Nat Rev Neurosci. 2002;3(3):237-243.

185. Shulman JM, Shulman LM, Weiner WJ, Feany MB. From fruit fly to bedside: translating lessons from Drosophila models of neurodegenerative disease. Curr Opin Neurol. 2003;16(4):443-449.

186. Greene JC, Whitworth AJ, Kuo I, Andrews LA, Feany MB, Pallanck LJ. Mitochondrial pathology and apoptotic muscle degeneration in Drosophila parkin mutants. Proceedings of the National Academy of Sciences of the United States of America. 2003;100(7):4078-4083.

187. Greene JC, Whitworth AJ, Andrews LA, Parker TJ, Pallanck LJ. Genetic and genomic studies of Drosophila parkin mutants implicate oxidative stress and innate immune responses in pathogenesis. Hum Mol Genet. 2005;14(6):799-811.

188. Pesah Y, Pham T, Burgess H, Middlebrooks B, Verstreken P, Zhou Y, Harding M, Bellen H, Mardon G. Drosophila parkin mutants have decreased mass and cell size and increased sensitivity to oxygen radical stress. Development. 2004;131(9):21832194.

189. Cha GH, Kim S, Park J, Lee E, Kim M, Lee SB, Kim JM, Chung J, Cho KS. Parkin negatively regulates JNK pathway in the dopaminergic neurons of Drosophila. Proc Natl Acad Sci U S A. 2005;102(29):10345-10350.

190. Meulener M, Whitworth AJ, Armstrong-Gold CE, Rizzu P, Heutink P, Wes PD, Pallanck LJ, Bonini NM. Drosophila DJ-1 mutants are selectively sensitive to environmental toxins associated with Parkinson's disease. Curr Biol. 2005;15(17):1572-1577.

191. Park J, Kim SY, Cha GH, Lee SB, Kim S, Chung J. Drosophila DJ-1 mutants show oxidative stress-sensitive locomotive dysfunction. Gene. 2005;361:133-139.

192. Liu Z, Wang X, Yu Y, Li X, Wang T, Jiang H, Ren Q, Jiao Y, Sawa A, Moran T, Ross CA, Montell C, Smith WW. A Drosophila model for LRRK2-linked parkinsonism. Proc Natl Acad Sci U S A. 2008;105(7):2693-2698.

193. Wang D, Tang B, Zhao G, Pan Q, Xia K, Bodmer R, Zhang Z. Dispensable role of Drosophila ortholog of LRRK2 kinase activity in survival of dopaminergic neurons. Mol Neurodegener. 2008;3:3.

194. Whitworth AJ, Lee JR, Ho VM, Flick R, Chowdhury R, McQuibban GA. Rhomboid-7 and HtrA2/Omi act in a common pathway with the Parkinson's disease factors Pink1 and Parkin. Dis Model Mech. 2008;1(2-3):168-174; discussion 173.

195. Tain LS, Chowdhury RB, Tao RN, Plun-Favreau H, Moisoi N, Martins LM, Downward J, Whitworth AJ, Tapon N. Drosophila HtrA2 is dispensable for apoptosis but acts downstream of PINK1 independently from Parkin. Cell Death Differ. 2009;16(8):1118-1125.

196. Yun J, Cao JH, Dodson MW, Clark IE, Kapahi P, Chowdhury RB, Guo M. Loss-offunction analysis suggests that Omi/HtrA2 is not an essential component of the PINK1/PARKIN pathway in vivo. J Neurosci. 2008;28(53):14500-14510. 
197. Auluck PK, Meulener MC, Bonini NM. Mechanisms of Suppression of \{alpha $\}$ Synuclein Neurotoxicity by Geldanamycin in Drosophila. J Biol Chem. 2005;280(4):2873-2878.

198. Haywood AF, Staveley BE. Mutant alpha-synuclein-induced degeneration is reduced by parkin in a fly model of Parkinson's disease. Genome. 2006;49(5):505-510.

199. Takahashi M, Kanuka H, Fujiwara H, Koyama A, Hasegawa M, Miura M, Iwatsubo T. Phosphorylation of alpha-synuclein characteristic of synucleinopathy lesions is recapitulated in alpha-synuclein transgenic Drosophila. Neurosci Lett. 2003;336(3):155-158.

200. Dufty BM, Warner LR, Hou ST, Jiang SX, Gomez-Isla T, Leenhouts KM, Oxford JT, Feany MB, Masliah E, Rohn TT. Calpain-cleavage of alpha-synuclein: connecting proteolytic processing to disease-linked aggregation. Am J Pathol. 2007;170(5):17251738.

201. Botella JA, Bayersdorfer F, Schneuwly S. Superoxide dismutase overexpression protects dopaminergic neurons in a Drosophila model of Parkinson's disease. Neurobiol Dis. 2008;30(1):65-73.

202. Shulman JM, Feany MB. Genetic modifiers of tauopathy in Drosophila. Genetics. 2003;165(3):1233-1242.

203. Karsten SL, Sang TK, Gehman LT, Chatterjee S, Liu J, Lawless GM, Sengupta S, Berry RW, Pomakian J, Oh HS, Schulz C, Hui KS, Wiedau-Pazos M, Vinters HV, Binder LI, Geschwind DH, Jackson GR. A genomic screen for modifiers of tauopathy identifies puromycin-sensitive aminopeptidase as an inhibitor of tau-induced neurodegeneration. Neuron. 2006;51(5):549-560.

204. Blard O, Feuillette S, Bou J, Chaumette B, Frebourg T, Campion D, Lecourtois M. Cytoskeleton proteins are modulators of mutant tau-induced neurodegeneration in Drosophila. Hum Mol Genet. 2007;16(5):555-566.

205. Tan L, Schedl P, Song HJ, Garza D, Konsolaki M. The Toll-->NFkappaB signaling pathway mediates the neuropathological effects of the human Alzheimer's Abeta42 polypeptide in Drosophila. PLoS One. 2008;3(12):e3966.

206. Sambrook J RD. Molecular Cloning: A Laboratory Manual Vol 2. 3rd ed. Cold Spring Harbour; USA: Cold Spring Harbour Laboratory Press; 2001.

207. Bischof J, Maeda RK, Hediger M, Karch Fo, Basler K. An optimized transgenesis system for Drosophila using germ-line-specific Ï†C31 integrases. Proceedings of the National Academy of Sciences. 2007;104(9):3312-3317.

208. Bonilla E, Medina-Leendertz S, Villalobos V, Molero L, Bohórquez A. Paraquatinduced Oxidative Stress in Drosophila melanogaster: Effects of Melatonin, Glutathione, Serotonin, Minocycline, Lipoic Acid and Ascorbic Acid. Neurochemical Research. 2006;31(12):1425-1432.

209. Du G, Liu X, Chen X, Song M, Yan Y, Jiao R, Wang C-c. Drosophila Histone Deacetylase 6 Protects Dopaminergic Neurons Against \{alpha\}-Synuclein Toxicity by Promoting Inclusion Formation. Mol. Biol. Cell.E10-03-0200.

210. Bayersdorfer F, Voigt A, Schneuwly S, Botella JA. Dopamine-dependent neurodegeneration in Drosophila models of familial and sporadic Parkinson's disease. Neurobiology of Disease.In Press, Corrected Proof.

211. Bertucci LA NM. Single fly total RNA preparations for RT-PCR. Drosophila Information Service. 2001;84:166-168.

212. Livak KJ, Schmittgen TD. Analysis of Relative Gene Expression Data Using RealTime Quantitative PCR and the 2-[Delta][Delta]CT Method. Methods. 2001;25(4):402-408. 
213. Büeler $H$. Impaired mitochondrial dynamics and function in the pathogenesis of Parkinson's disease. Experimental Neurology.In Press, Accepted Manuscript.

214. Gegg ME, Cooper JM, Schapira AHV, Taanman J-W. Silencing of PINK1 Expression Affects Mitochondrial DNA and Oxidative Phosphorylation in DOPAMINERGIC Cells. PLoS ONE. 2009;4(3):e4756.

215. Hirsh J, Riemensperger T, Coulom H, Iché M, Coupar J, Birman S. Roles of Dopamine in Circadian Rhythmicity and Extreme Light Sensitivity of Circadian Entrainment. Current Biology. 2010;20(3):209-214.

216. Deng H, Dodson MW, Huang H, Guo M. The Parkinson's disease genes pink1 and parkin promote mitochondrial fission and/or inhibit fusion in Drosophila. Proc Natl Acad Sci U S A. 2008;105(38):14503-14508.

217. Poole AC, Thomas RE, Andrews LA, McBride HM, Whitworth AJ, Pallanck LJ. The PINK1/Parkin pathway regulates mitochondrial morphology. Proceedings of the National Academy of Sciences. 2008;105(5):1638-1643.

218. Zhou W, Freed CR. DJ-1 Up-regulates Glutathione Synthesis during Oxidative Stress and Inhibits A53T Î+-Synuclein Toxicity. Journal of Biological Chemistry. 2005;280(52):43150-43158.

219. Chen L, Periquet M, Wang X, Negro A, McLean PJ, Hyman BT, Feany MB. Tyrosine and serine phosphorylation of $\hat{\mathrm{I}} \pm$-synuclein have opposing effects on neurotoxicity and soluble oligomer formation. The Journal of Clinical Investigation. 2009;119(11):3257-3265.

220. Francesca KML, Azaria KYW, Yuk Wa L, Oi Wan W, Chan HYE, Kenny KKC. The role of ubiquitin linkages on \&\#x03B1;-synuclein induced-toxicity in a $<\mathrm{i}>$ Drosophila $</ \mathrm{i}>$ model of Parkinson's disease. Journal of Neurochemistry. 2009;110(1):208-219.

221. Yakov P, Heather B, Brooke M, Kari R, Jude P, Vijaya T, John Z, Graeme M. Wholemount analysis reveals normal numbers of dopaminergic neurons following misexpression of $\langle\mathrm{I}>$ alpha-Synuclein $</ \mathrm{I}\rangle$ in $\langle\mathrm{I}\rangle$ Drosophila $</ \mathrm{I}\rangle$. genesis. 2005;41(4):154-159.

222. Coulom H, Birman S. Chronic Exposure to Rotenone Models Sporadic Parkinson's Disease in Drosophila melanogaster. J. Neurosci. 2004;24(48):10993-10998.

223. .

224. Bellen HJ, Levis RW, Liao G, He Y, Carlson JW, Tsang G, Evans-Holm M, Hiesinger PR, Schulze KL, Rubin GM, Hoskins RA, Spradling AC. The BDGP gene disruption project: single transposon insertions associated with $40 \%$ of Drosophila genes. Genetics. 2004;167(2):761-781.

225. Chaudhuri A, Bowling K, Funderburk C, Lawal H, Inamdar A, Wang Z, O'Donnell JM. Interaction of Genetic and Environmental Factors in a Drosophila Parkinsonism Model. J. Neurosci. 2007;27(10):2457-2467.

226. Ramachandiran S, Hansen JM, Jones DP, Richardson JR, Miller GW. Divergent Mechanisms of Paraquat, MPP+, and Rotenone Toxicity: Oxidation of Thioredoxin and Caspase-3 Activation. Toxicol. Sci. 2007;95(1):163-171.

227. Miquel V, Slobodanka V, Vernice J-L, Michael N, Michael J, Serge P. \&\#x03B1;Synuclein Up-Regulation in Substantia Nigra Dopaminergic Neurons Following Administration of the Parkinsonian Toxin MPTP. Journal of Neurochemistry. 2000;74(2):721-729.

228. Sherer TB, Betarbet R, Stout AK, Lund S, Baptista M, Panov AV, Cookson MR, Greenamyre JT. An In Vitro Model of Parkinson's Disease: Linking Mitochondrial Impairment to Altered alpha -Synuclein Metabolism and Oxidative Damage. $J$. Neurosci. 2002;22(16):7006-7015. 
229. Hua G, Zhang Q, Fan Z. Heat shock protein 75 (TRAP1) antagonizes reactive oxygen species generation and protects cells from granzyme M-mediated apoptosis. $J$ Biol Chem. 2007;282(28):20553-20560.

230. Masuda Y, Shima G, Aiuchi T, Horie M, Hori K, Nakajo S, Kajimoto S, ShibayamaImazu T, Nakaya K. Involvement of tumor necrosis factor receptor-associated protein 1 (TRAP1) in apoptosis induced by beta-hydroxyisovalerylshikonin. J Biol Chem. 2004;279(41):42503-42515.

231. Chen L, Xu B, Liu L, Luo Y, Yin J, Zhou H, Chen W, Shen T, Han X, Huang S. Hydrogen peroxide inhibits mTOR signaling by activation of AMPKalpha leading to apoptosis of neuronal cells. Lab Invest.90(5):762-773.

232. Eleonora C, Francesca M, Serena C, Annamaria P, Virginia T, Alberto F, Antonio A, Vincenzo N, Franca E, Matteo L. TRAP1, a novel mitochondrial chaperone responsible for multi-drug resistance and protection from apoptotis in human colorectal carcinoma cells. Cancer letters. 2009;279(1):39-46.

233. Ruiz-Romero C, Calamia V, Mateos J, Carreira V, Martinez-Gomariz M, Fernandez M, Blanco FJ. Mitochondrial Dysregulation of Osteoarthritic Human Articular Chondrocytes Analyzed by Proteomics: A Decrease in Mitochondrial Superoxide Dismutase Points to a Redox Imbalance. Mol Cell Proteomics. 2009;8(1):172-189.

234. Gesualdi NM, Chirico G, Pirozzi G, Costantino E, Landriscina M, Esposito F. Tumor necrosis factor-associated protein 1 (TRAP-1) protects cells from oxidative stress and apoptosis. Stress: The International Journal on the Biology of Stress. 2007;10(4):342350.

235. Buttner S, Bitto A, Ring J, Augsten M, Zabrocki P, Eisenberg T, Jungwirth H, Hutter S, Carmona-Gutierrez D, Kroemer G, Winderickx J, Madeo F. Functional mitochondria are required for alpha-synuclein toxicity in aging yeast. $J$ Biol Chem. 2008;283(12):7554-7560.

236. Xiang F, Huang YS, Shi XH, Zhang Q. Mitochondrial chaperone tumour necrosis factor receptor-associated protein 1 protects cardiomyocytes from hypoxic injury by regulating mitochondrial permeability transition pore opening. FEBS J.277(8):19291938.

237. Masuda Y, Shima G, Aiuchi T, Horie M, Hori K, Nakajo S, Kajimoto S, ShibayamaImazu T, Nakaya K. Involvement of Tumor Necrosis Factor Receptor-associated Protein 1 (TRAP1) in Apoptosis Induced by $\hat{\mathbf{I}}^{2}$-Hydroxyisovalerylshikonin. Journal of Biological Chemistry. 2004;279(41):42503-42515.

238. Kanda S, Bishop JF, Eglitis MA, Yang Y, Mouradian MM. Enhanced vulnerability to oxidative stress by alpha-synuclein mutations and C-terminal truncation. Neuroscience. 2000;97(2):279-284.

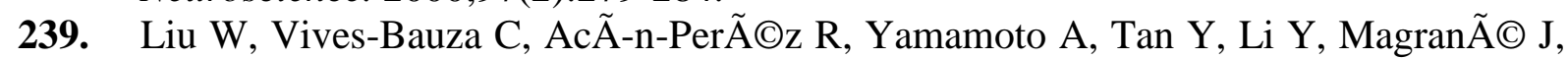
Stavarache MA, Shaffer S, Chang S, Kaplitt MG, Huang X-Y, Beal MF, Manfredi G, Li C. PINK1 Defect Causes Mitochondrial Dysfunction, Proteasomal Deficit and $\hat{\mathrm{I}} \pm-$ Synuclein Aggregation in Cell Culture Models of Parkinson's Disease. PLoS ONE. 2009;4(2):e4597.

240. Barrientos A, Moraes CT. Titrating the Effects of Mitochondrial Complex I Impairment in the Cell Physiology. Journal of Biological Chemistry. 1999;274(23):16188-16197.

241. Panaretou B, Prodromou C, Roe SM, O'Brien R, Ladbury JE, Piper PW, Pearl LH. ATP binding and hydrolysis are essential to the function of the Hsp90 molecular chaperone in vivo. EMBO J. 1998;17(16):4829-4836. 
242. Chen J, Shi X, Padmanabhan R, Wang Q, Wu Z, Stevenson SC, Hild M, Garza D, Li $\mathrm{H}$. Identification of novel modulators of mitochondrial function by a genome-wide RNAi screen in Drosophila melanogaster. Genome Res. 2008;18(1):123-136.

243. Brown AM, Baucum AJ, Bass MA, Colbran RJ. Association of Protein Phosphatase $1 \hat{\mathrm{I}}^{3} 1$ with Spinophilin Suppresses Phosphatase Activity in a Parkinson Disease Model. Journal of Biological Chemistry. 2008;283(21):14286-14294.

244. Svenningsson P, Nishi A, Fisone G, Girault J-A, Nairn AC, Greengard P. DARPP-32: An Integrator of Neurotransmission. Annual Review of Pharmacology and Toxicology. 2004;44(1):269-296.

245. Palvimo JJ. PIAS proteins as regulators of small ubiquitin-related modifier (SUMO) modifications and transcription. Biochemical Society Transactions. 2007;035(6):14051408 .

246. Aerts S, Vilain S, Hu S, Tranchevent L-C, Barriot R, Yan J, Moreau Y, Hassan BA, Quan X-J. Integrating Computational Biology and Forward Genetics in <italic>Drosophila</italic>. PLoS Genet. 2009;5(1):e1000351.

247. Price DM, Jin Z, Rabinovitch S, Campbell SD. Ectopic Expression of the Drosophila Cdk1 Inhibitory Kinases, Wee1 and Myt1, Interferes With the Second Mitotic Wave and Disrupts Pattern Formation During Eye Development. Genetics. 2002;161(2):721731.

248. Moyes CD, Mathieu-Costello OA, Tsuchiya N, Filburn C, Hansford RG. Mitochondrial biogenesis during cellular differentiation. Am J Physiol. 1997;272(4 Pt 1):C1345-1351.

249. Bruce CR, Kriketos AD, Cooney GJ, Hawley JA. Disassociation of muscle triglyceride content and insulin sensitivity after exercise training in patients with Type 2 diabetes. Diabetologia. 2004;47(1):23-30.

250. Liang J, Clark-Dixon C, Wang S, Flower TR, Williams-Hart T, Zweig R, Robinson LC, Tatchell K, Witt SN. Novel suppressors of alpha-synuclein toxicity identified using yeast. Hum Mol Genet. 2008;17(23):3784-3795.

251. Pennington K, Peng J, Hung CC, Banks RE, Robinson PA. Differential effects of wild-type and A53T mutant isoform of alpha-synuclein on the mitochondrial proteome of differentiated SH-SY5Y cells. J Proteome Res.9(5):2390-2401.

252. Qian J-J, Cheng Y-B, Yang Y-P, Mao C-J, Qin Z-H, Li K, Liu C-F. Differential effects of overexpression of wild-type and mutant human [alpha]-synuclein on MPP+induced neurotoxicity in PC12 cells. Neuroscience Letters. 2008;435(2):142-146.

253. Qian JJ, Cheng YB, Yang YP, Mao CJ, Qin ZH, Li K, Liu CF. Differential effects of overexpression of wild-type and mutant human alpha-synuclein on MPP+-induced neurotoxicity in PC12 cells. Neurosci Lett. 2008;435(2):142-146.

254. Flower TR, Clark-Dixon C, Metoyer C, Yang H, Shi R, Zhang Z, Witt SN. YGR198w (YPP1) targets A30P $\hat{\mathrm{I}} \pm$-synuclein to the vacuole for degradation. The Journal of Cell Biology. 2007; 177(6):1091-1104.

255. Outeiro TF, Kontopoulos E, Altmann SM, Kufareva I, Strathearn KE, Amore AM, Volk CB, Maxwell MM, Rochet JC, McLean PJ, Young AB, Abagyan R, Feany MB, Hyman BT, Kazantsev AG. Sirtuin 2 inhibitors rescue alpha-synuclein-mediated toxicity in models of Parkinson's disease. Science. 2007;317(5837):516-519.

256. Hamamichi S, Rivas RN, Knight AL, Cao S, Caldwell KA, Caldwell GA. Hypothesisbased RNAi screening identifies neuroprotective genes in a Parkinson's disease model. Proc Natl Acad Sci U S A. 2008;105(2):728-733.

257. Willingham S, Outeiro TF, DeVit MJ, Lindquist SL, Muchowski PJ. Yeast genes that enhance the toxicity of a mutant huntingtin fragment or alpha-synuclein. Science. 2003;302(5651):1769-1772. 
258. Sugiyama S, Moritoh S, Furukawa $Y$, Mizuno T, Lim Y-M, Tsuda L, Nishida $Y$. Involvement of the Mitochondrial Protein Translocator Component Tim50 in Growth, Cell Proliferation and the Modulation of Respiration in Drosophila. Genetics. 2007;176(2):927-936.

259. Scherzer CR, Jensen RV, Gullans SR, Feany MB. Gene expression changes presage neurodegeneration in a Drosophila model of Parkinson's disease. Hum Mol Genet. 2003;12(19):2457-2466.

260. Xun Z, Sowell RA, Kaufman TC, Clemmer DE. Quantitative proteomics of a presymptomatic A53T alpha-synuclein Drosophila model of Parkinson disease. Mol Cell Proteomics. 2008;7(7):1191-1203.

261. Xun Z, Sowell RA, Kaufman TC, Clemmer DE. Lifetime proteomic profiling of an A30P alpha-synuclein Drosophila model of Parkinson's disease. J Proteome Res. 2007;6(9):3729-3738.

262. Talamillo A, Sanchez J, Barrio R. Functional analysis of the SUMOylation pathway in Drosophila. Biochem Soc Trans. 2008;36(Pt 5):868-873.

263. Dorval V, Fraser PE. SUMO on the road to neurodegeneration. Biochim Biophys Acta. 2007;1773(6):694-706.

264. Dorval V, Fraser PE. Small ubiquitin-like modifier (SUMO) modification of natively unfolded proteins tau and alpha-synuclein. J Biol Chem. 2006;281(15):9919-9924.

265. Scott RC, Schuldiner O, Neufeld TP. Role and Regulation of Starvation-Induced Autophagy in the Drosophila Fat Body. Developmental Cell. 2004;7(2):167-178.

266. Patel PH, Tamanoi F. Increased Rheb-TOR signaling enhances sensitivity of the whole organism to oxidative stress. Vol 119; 2006:4285-4292.

267. Todd AM, Staveley BE. Pink1 suppresses alpha-synuclein-induced phenotypes in a Drosophila model of Parkinson's disease. Genome. 2008;51(12):1040-1046.

268. Sang TK, Chang HY, Lawless GM, Ratnaparkhi A, Mee L, Ackerson LC, Maidment NT, Krantz DE, Jackson GR. A Drosophila model of mutant human parkin-induced toxicity demonstrates selective loss of dopaminergic neurons and dependence on cellular dopamine. J Neurosci. 2007;27(5):981-992.

269. Costantino E, Maddalena F, Calise S, Piscazzi A, Tirino V, Fersini A, Ambrosi A, Neri V, Esposito F, Landriscina M. TRAP1, a novel mitochondrial chaperone responsible for multi-drug resistance and protection from apoptotis in human colorectal carcinoma cells. Cancer Lett. 2009;279(1):39-46.

270. Nagley P, Higgins GC, Atkin JD, Beart PM. Multifaceted deaths orchestrated by mitochondria in neurones. Biochim Biophys Acta.1802(1):167-185.

271. Hwang S, Kim D, Choi G, An SW, Hong YK, Suh YS, Lee MJ, Cho KS. Parkin Suppresses c-Jun N-terminal kinase-induced cell death via transriptional regulation in Drosophila. Mol Cells.

272. Novikova L, Garris BL, Garris DR, Lau YS. Early signs of neuronal apoptosis in the substantia nigra pars compacta of the progressive neurodegenerative mouse 1-methyl4-phenyl-1,2,3,6-tetrahydropyridine/probenecid model of Parkinson's disease. Neuroscience. 2006;140(1):67-76.

273. Singh S, Kumar S, Dikshit M. Involvement of the mitochondrial apoptotic pathway and nitric oxide synthase in dopaminergic neuronal death induced by 6hydroxydopamine and lipopolysaccharide. Redox Rep.15(3):115-122.

274. Choi WS, Eom DS, Han BS, Kim WK, Han BH, Choi EJ, Oh TH, Markelonis GJ, Cho JW, Oh YJ. Phosphorylation of p38 MAPK induced by oxidative stress is linked to activation of both caspase-8- and -9-mediated apoptotic pathways in dopaminergic neurons. J Biol Chem. 2004;279(19):20451-20460. 
275. Ramachandiran S, Hansen JM, Jones DP, Richardson JR, Miller GW. Divergent mechanisms of paraquat, MPP+, and rotenone toxicity: oxidation of thioredoxin and caspase-3 activation. Toxicol Sci. 2007;95(1):163-171.

276. Fukushima T. [Elucidation of paraquat poisoning mechanism and development of the neuronal death model]. Nippon Eiseigaku Zasshi. 2002;57(1):83-86.

277. Albani D, Peverelli E, Rametta R, Batelli S, Veschini L, Negro A, Forloni G. Protective effect of TAT-delivered alpha-synuclein: relevance of the C-terminal domain and involvement of HSP70. FASEB J. 2004;18(14):1713-1715.

278. Bandhyopadhyay U, Cuervo AM. Chaperone-mediated autophagy in aging and neurodegeneration: lessons from alpha-synuclein. Exp Gerontol. 2007;42(1-2):120128.

279. Jiang H, Wu Y-C, Nakamura M, Liang Y, Tanaka Y, Holmes S, Dawson VL, Dawson TM, Ross CA, Smith WW. Parkinson's disease genetic mutations increase cell susceptibility to stress: Mutant [alpha]-synuclein enhances H2O2- and Sin-1-induced cell death. Neurobiology of Aging. 2007;28(11):1709-1717.

280. Roiniotis J, Dinh H, Masendycz P, Turner A, Elsegood CL, Scholz GM, Hamilton JA. Hypoxia Prolongs Monocyte/Macrophage Survival and Enhanced Glycolysis Is Associated with Their Maturation under Aerobic Conditions. $J$ Immunol. 2009;182(12):7974-7981.

281. Maher JC, Wangpaichitr M, Savaraj N, Kurtoglu M, Lampidis TJ. Hypoxia-inducible factor-1 confers resistance to the glycolytic inhibitor 2-deoxy-d-glucose. Molecular Cancer Therapeutics. 2007;6(2):732-741.

282. Vahsen N, Cande C, Briere JJ, Benit P, Joza N, Larochette N, Mastroberardino PG, Pequignot MO, Casares N, Lazar V, Feraud O, Debili N, Wissing S, Engelhardt S, Madeo F, Piacentini M, Penninger JM, Schagger H, Rustin P, Kroemer G. AIF deficiency compromises oxidative phosphorylation. EMBO J. 2004;23(23):4679-4689.

283. Campanella M, Casswell E, Chong S, Farah Z, Wieckowski MR, Abramov AY, Tinker A, Duchen MR. Regulation of mitochondrial structure and function by the F1Fo-ATPase inhibitor protein, IF1. Cell Metab. 2008;8(1):13-25.

284. Voloboueva LA, Duan M, Ouyang Y, Emery JF, Stoy C, Giffard RG. Overexpression of mitochondrial Hsp70/Hsp75 protects astrocytes against ischemic injury in vitro. $J$ Cereb Blood Flow Metab. 2008;28(5):1009-1016.

285. Moro F, Okamoto K, Donzeau M, Neupert W, Brunner M. Mitochondrial protein import: molecular basis of the ATP-dependent interaction of MtHsp70 with Tim44. J Biol Chem. 2002;277(9):6874-6880.

286. Caplan AJ. What is a co-chaperone? Cell Stress Chaperones. 2003;8(2):105-107.

287. http://www.phosphosite.org/proteinAction.do?id=4177\&showAllSites=false.

288. Morais VA, Verstreken P, Roethig A, Smet J, Snellinx A, Vanbrabant M, Haddad D, Frezza C, Mandemakers W, Vogt-Weisenhorn D, Van Coster R, Wurst W, Scorrano L, De Strooper B. Parkinson's disease mutations in PINK1 result in decreased Complex I activity and deficient synaptic function. EMBO Mol Med. 2009;1(2):99111. 
